\title{
Stereocontrolled Synthesis and Structural Revision of Plebeianiol A
}

Lucas K. Johnson ${ }^{1 \S}$, Scott W. Niman ${ }^{1 \S}$, Darius Vrubliauskas ${ }^{1}$, and Christopher D. Vanderwal ${ }^{1,2, *}$

${ }^{1}$ Department of Chemistry, 1102 Natural Sciences II, University of California, Irvine, CA 92697-2025, USA.

${ }^{2}$ Department of Pharmaceutical Sciences, 101 Theory \#100, University of California, Irvine, CA 92617, USA.

*cdv@uci.edu 


\section{Supporting Information}

Table of Contents:

\section{Experimental Information:}

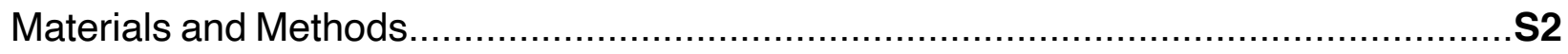

Experimental Procedures and Characterization Data....................................................

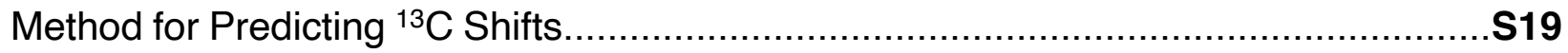

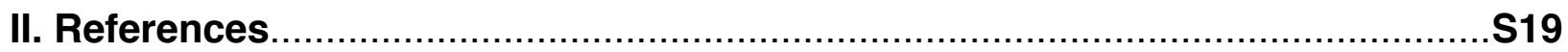

III. Experimental Data:

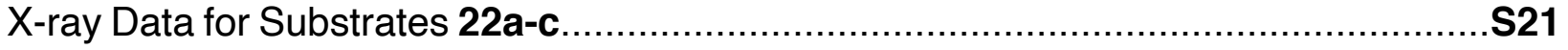

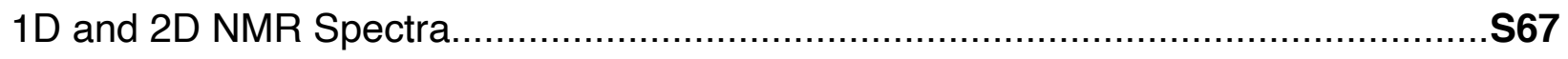

Predicted ${ }^{13} \mathrm{C}$ Shifts and Comparisons of Spectral Data............................................118 


\section{Experimental Information}

\section{Materials and Methods}

All reactions were performed in oven-dried $\left(120^{\circ} \mathrm{C}\right)$ or flame-dried glassware under an atmosphere of dry argon unless otherwise noted. Reaction solvents including dichloromethane $\left(\mathrm{CH}_{2} \mathrm{Cl}_{2}\right.$, Fisher, HPLC Grade), hexanes (Fisher, HPLC Grade), diethyl ether ( $\mathrm{Et}_{2} \mathrm{O}$, Fisher, BHT stabilized, HPLC Grade), benzene $\left(\mathrm{C}_{6} \mathrm{H}_{6}\right.$, Fisher, HPLC Grade), tetrahydrofuran (THF, Fisher, HPLC Grade), and toluene ( $\mathrm{PhCH}_{3}$, Fisher, HPLC Grade) were dried by percolation through a column packed with neutral alumina and a column packed with Q5 reactant, a supported copper catalyst for scavenging oxygen, under a positive pressure of argon.

Solvents for workup and chromatography were: hexanes (Fisher or EMD, ACS Grade), EtOAc (Fisher, ACS Grade), dichloromethane $\left(\mathrm{CH}_{2} \mathrm{Cl}_{2}\right.$, Fisher, ACS Grade), and diethyl ether (Fisher, ACS Grade). Column chromatography was performed using EMD Millipore $60 \AA$ (0.040-0.063 mm) mesh silica gel $\left(\mathrm{SiO}_{2}\right)$. Analytical and preparatory thinlayer chromatography was performed on Merck silica gel 60 F254 TLC plates. Visualization was accomplished with UV (254 or $210 \mathrm{~nm}$ ), and p-anisaldehyde, vanillin, potassium permanganate, 2,4-dinitrophenylhydrazine, or ceric ammonium molybdate and heat as developing agents. Chloroform-d $\left(\mathrm{CDCl}_{3}, 99.8 \% \mathrm{D}, \mathrm{DLM}-7\right)$ and Dimethyl sulfoxide-D6 (99.9\% D, DLM-10) were purchased from Cambridge Isotope Laboratories. $\mathrm{K}_{2} \mathrm{CO}_{3}$ (anhydrous, 99\%, Alfa Aesar), $\mathrm{NaHCO}_{3}$ (ACS grade, Fisher), $\mathrm{NaOH}$ (ACS grade, Macron or Fisher), $\mathrm{Na}_{2} \mathrm{~S}_{2} \mathrm{O}_{3}$ (ACS grade, Fisher), 1-Fluoro-2,4,6-trimethylpyridinium tetrafluoroborate ( $\mathrm{TCl}$ or Sigma Aldrich, 95-99\%) were purchased and used without further purification. Triethylamine $\left(\mathrm{EMD}, \mathrm{CaH}_{2}\right)$ and pyridine $\left(\mathrm{Alfa} \mathrm{Aesar}, \mathrm{CaH}_{2}\right)$ were distilled from the indicated drying agents prior to use.

Proton and carbon magnetic resonance spectra ( ${ }^{1} \mathrm{H}$ NMR and $\left.{ }^{13} \mathrm{C} N M R\right)$ were recorded at $298 \mathrm{~K}$ on a Bruker CRYO500 $\left(500 \mathrm{MHz},{ }^{1} \mathrm{H} ; 125 \mathrm{MHz},{ }^{13} \mathrm{C}\right)$ or a Bruker AVANCE600 $\left(600 \mathrm{MHz},{ }^{1} \mathrm{H} ; 151 \mathrm{MHz},{ }^{13} \mathrm{C}\right)$ spectrometer with solvent residual as the internal standard for ${ }^{1} \mathrm{H}$ NMR: $\mathrm{CHCl}_{3}$ at $7.27 \mathrm{ppm}$, and solvent as internal standard for ${ }^{13} \mathrm{C}$ NMR: $\mathrm{CDCl}_{3}$ at $77.16 \mathrm{ppm} .{ }^{1} \mathrm{H}$ NMR data are reported as follows: chemical shift, multiplicity $(\mathrm{s}=$ singlet, $\mathrm{d}=$ doublet, $\mathrm{t}=$ triplet, $\mathrm{q}=$ quartet, $\mathrm{dd}=$ doublet of doublets, $\mathrm{ddd}$ 
$=$ doublet of doublet of doublets, $\mathrm{td}=$ triplet of doublets, $\mathrm{tdd}=$ triplet of doublet of doublets, $q d=$ quartet of doublets, $m=$ multiplet, br. s. = broad singlet), coupling constants $(\mathrm{Hz})$, and integration. High resolution mass spectra (HRMS) were recorded on a Waters LCT Premier S2 spectrometer using ESI-TOF (electrospray ionization-time of flight) and data are reported in the form of $(\mathrm{m} / \mathrm{z})$. Optical rotation was measured on a Jasco P-1010 polarimeter with an optical path of $5 \mathrm{~cm}$ at $22-25^{\circ} \mathrm{C}$ with the measurement performed in $\mathrm{CHCl}_{3}$.

Note: Significant reaction optimization was performed on material produced from Sharpless dihydroxylation with $(D H Q)_{2} P H A L$ because the $2 R, 3 S$-diol was needed for another synthesis project in our group. In some cases, spectroscopic data were collected on substrates from this enantiomeric series, and that is noted where relevant. The $2 S, 3 R$ diol for our work toward plebeianiol $A$ was obtained with $(D H Q D)_{2} P H A L$ as the chiral ligand.

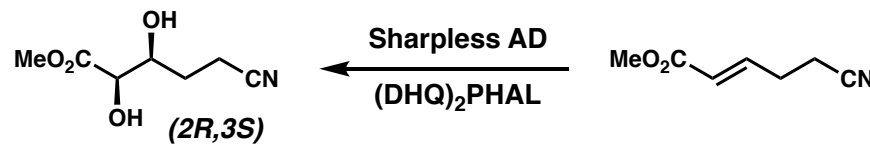

for use in another total synthesis

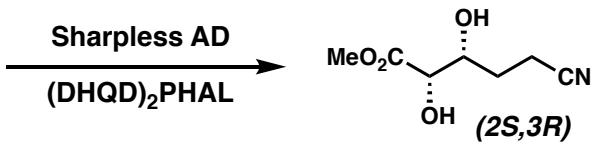

for use toward plebeianiol A 


\section{Experimental Procedures and Characterization Data}

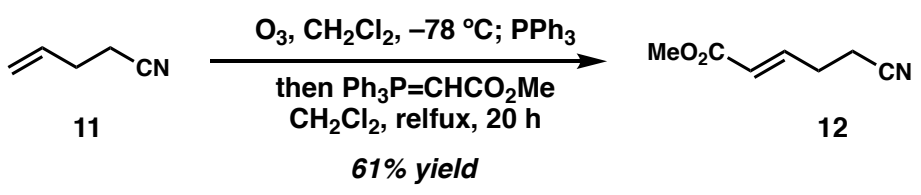

To a $500 \mathrm{~mL}$ round-bottomed flask was added 4-pentenenitrile $(\mathbf{1 1})^{1}(5.0 \mathrm{~g}, 61.6 \mathrm{mmol}$, 1.0 equiv.) and $\mathrm{CH}_{2} \mathrm{Cl}_{2}(185 \mathrm{~mL})$ at room temperature, open to air. This solution was cooled to $-78^{\circ} \mathrm{C}$ and ozone was bubbled through the mixture until a blue color persisted (ca. 30 minutes). Oxygen was bubbled until the solution became colorless (ca. 20 minutes) and then $\mathrm{PPh}_{3}(16.98 \mathrm{~g}, 64.7 \mathrm{mmol}, 1.05$ equiv.) was added in one portion at $78{ }^{\circ} \mathrm{C}$. After 5 minutes, the cooling bath was removed and methyl (triphenylphosphoranylidene)acetate $(28.8 \mathrm{~g}, 86.3 \mathrm{mmol})$ in $\mathrm{CH}_{2} \mathrm{Cl}_{2}(100 \mathrm{~mL})$ was added slowly. After 1 hour, the flask was fitted with a reflux condenser and submerged in a preheated $50^{\circ} \mathrm{C}$ oil bath. After 20 hours, the mixture was cooled to room temperature and

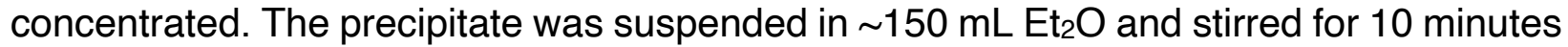

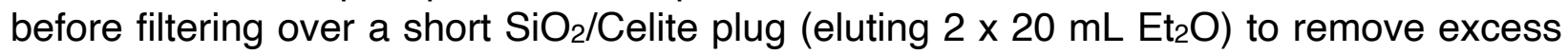
triphenylphosphine oxide. The filtrate was concentrated and the resulting residue was purified by column chromatography $\left(\mathrm{SiO}_{2}, 5: 1\right.$ then $1: 1$ hexanes:EtOAc) to afford acrylate 12 as a pale yellow-orange oil $(4.95 \mathrm{~g})$ and some additional product contaminated with triphenylphosphine oxide (purified as above to give $300 \mathrm{mg}$ of additional 12). The combined yield was $5.25 \mathrm{~g}$ (61\% yield).

${ }^{1} \mathrm{H}$ NMR $\left(600 \mathrm{MHz}, \mathrm{CDCl}_{3}\right) \delta 6.92(\mathrm{dt}, \mathrm{J}=15.7,6.6 \mathrm{~Hz}, 1 \mathrm{H}), 5.96(\mathrm{dt}, \mathrm{J}=15.6,1.6 \mathrm{~Hz}$, $1 \mathrm{H}$ ), $3.76(\mathrm{~s}, 3 \mathrm{H}), 2.58$ (qt, J = 6.6, $1.5 \mathrm{~Hz}, 2 \mathrm{H}$ ), 2.52 (td, J = 6.8, $1.4 \mathrm{~Hz}, 2 \mathrm{H}$ ).

${ }^{13} \mathrm{C}$ NMR $\left(151 \mathrm{MHz}, \mathrm{CDCl}_{3}\right): \delta 166.3,143.5,123.9,118.5,51.9,27.9,16.4$.

HRMS (Cl+) $m / z$ calc'd for $\mathrm{C}_{7} \mathrm{H}_{9} \mathrm{NO}_{2}\left[\mathrm{M}+\mathrm{NH}_{4}\right]^{+}: 157.0977$, found 157.0978 .

TLC: $\mathrm{R}_{f}=0.24$ (3:1 hexanes:EtOAc, visualized by $\mathrm{KMnO}_{4}$ ).

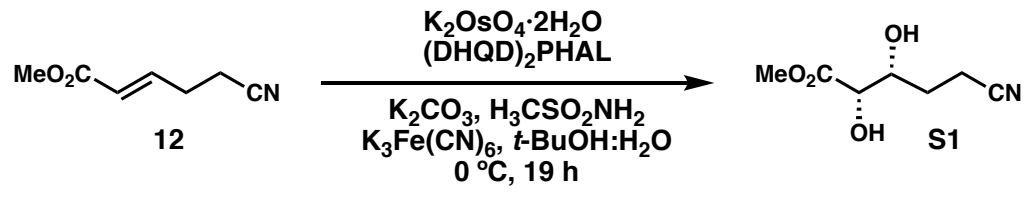

To a $100 \mathrm{~mL}$ round-bottomed flask at room temperature and open to air was added $\mathrm{K}_{2} \mathrm{OsO}_{4} \cdot 2 \mathrm{H}_{2} \mathrm{O}$ (13.2 mg, $0.0359 \mathrm{mmol}, 0.01$ equiv), (DHQD) ${ }_{2} \mathrm{PHAL}$ (28.0 mg, 0.0359 mmol, 0.01 equiv), $\mathrm{H}_{3} \mathrm{CSO}_{2} \mathrm{NH}_{2}$ (341 mg, 3.59 mmol, 1.0 equiv.), $\mathrm{K}_{2} \mathrm{CO}_{3}$ (1.49 g, 10.77 mmol, 3.0 equiv.), and $\mathrm{K}_{3} \mathrm{Fe}(\mathrm{CN})_{6}(3.55 \mathrm{~g}, 10.77 \mathrm{mmol}, 3.0$ equiv.). The solid reagents were dissolved in $\mathrm{H}_{2} \mathrm{O}(18 \mathrm{~mL})$ and $t-\mathrm{BuOH}(14 \mathrm{~mL})$ and cooled to $0{ }^{\circ} \mathrm{C}$. After 10 minutes at this temperature, the alkene $12(500 \mathrm{mg}, 3.59 \mathrm{mmol}, 1.0$ equiv.) dissolved in $t$-BuOH $(3 \mathrm{~mL})$ was added slowly followed by a $t-\mathrm{BuOH}$ rinse $(1 \mathrm{~mL})$. The heterogeneous mixture was stirred vigorously at $0^{\circ} \mathrm{C}$ until complete by TLC (ca. 19 hours). The reaction was quenched with $\mathrm{Na}_{2} \mathrm{SO}_{3}(\sim 300 \mathrm{mg})$ and allowed to warm to room temperature with 
vigorous stirring for 45 minutes. The solution was transferred to a separatory funnel and extracted with EtOAc $(8 \times 20 \mathrm{~mL})$. The combined organics were dried over $\mathrm{MgSO}_{4}$, filtered and concentrated in vacuo. The resulting crude residue $(910 \mathrm{mg})$ was used in the next reaction without further purification. A small portion of the crude reaction mixture was purified by column chromatography $\left(\mathrm{SiO}_{2}, 95: 5 \mathrm{CH}_{2} \mathrm{Cl}_{2}: \mathrm{MeOH}\right)$ for analytical purposes (the enantiomeric excess was estimated to be $94 \%$ by Mosher's ester analysis; see below). The absolute configuration of diol $\mathbf{S 1}$ was assumed based on the mnemonic rule for the facial selectivity of the Sharpless dihydroxylation reaction. ${ }^{2}$

${ }^{1} \mathrm{H}$ NMR $\left(600 \mathrm{MHz}, \mathrm{CDCl}_{3}\right) \delta 4.12(\mathrm{dd}, J=4.7,2.3 \mathrm{~Hz}, 1 \mathrm{H}), 4.05(\mathrm{t}, J=8.5 \mathrm{~Hz}, 1 \mathrm{H}), 3.87$ (s, 3H), 3.19 (d, $J=4.9 \mathrm{~Hz}, 1 \mathrm{H}), 2.70-2.43(\mathrm{~m}, 2 \mathrm{H}), 2.26(\mathrm{~d}, J=8.9 \mathrm{~Hz}, 1 \mathrm{H}), 2.05-1.85$ $(\mathrm{m}, 2 \mathrm{H})$.

${ }^{13} \mathrm{C}$ NMR $\left(151 \mathrm{MHz}, \mathrm{CDCl}_{3}\right): \delta 173.3,119.5,73.1,70.6,53.3,29.7,13.9$.

HRMS (ES+) $\mathrm{m} / \mathrm{z}$ calc'd for $\mathrm{C}_{7} \mathrm{H}_{11} \mathrm{NO}_{4}[\mathrm{M}+\mathrm{Na}]^{+:}$196.0586, found 196.0581 . $[\alpha]^{21.3} \mathrm{D}+45.8\left(\mathrm{c}=1.01, \mathrm{CHCl}_{3}\right)$.

TLC: $\mathrm{R}_{f}=0.43\left(\right.$ EtOAc, visualized by $\left.\mathrm{KMnO}_{4}\right)$.

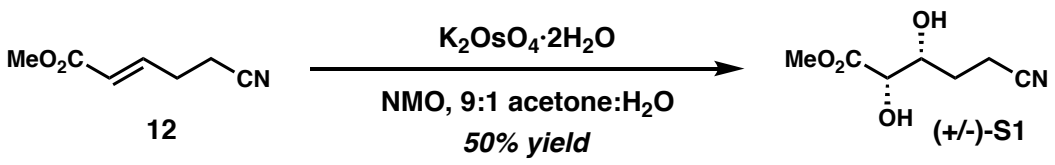

To a room temperature solution of acrylate $12(310 \mathrm{mg}, 2.23 \mathrm{mmol})$ in 9:1 acetone: $\mathrm{H}_{2} \mathrm{O}$ $(11.1 \mathrm{~mL}, 0.20 \mathrm{M})$ in a $20 \mathrm{~mL}$ vial was added $\mathrm{NMO} \cdot \mathrm{H}_{2} \mathrm{O}(603 \mathrm{mg}, 4.46 \mathrm{mmol}, 2.0$ equiv.). $\mathrm{K}_{2} \mathrm{OsO}_{4} \cdot 2 \mathrm{H}_{2} \mathrm{O}$ (16.2 mg, $0.044 \mathrm{mmol}, 0.02$ equiv.) was added in one portion open to air and the vial was capped and allowed to react overnight. After 20 hours, the reaction was quenched with saturated aqueous sodium thiosulfate $(5 \mathrm{~mL})$ and the mixture was extracted with $\mathrm{CH}_{2} \mathrm{Cl}_{2}(5 \times 10 \mathrm{~mL})$. The combined organic extracts were dried over $\mathrm{MgSO}_{4}$, filtered, and concentrated in vacuo. The resulting crude residue was purified by column chromatography over a short plug $\left(\mathrm{SiO}_{2}, \mathrm{EtOAc}\right)$ to afford rac-S1 as a colorless solid (195 mg, 50\% yield). The spectroscopic data matched those of the diol $\mathbf{S 1}$ made by Sharpless asymmetric dihydroxylation.
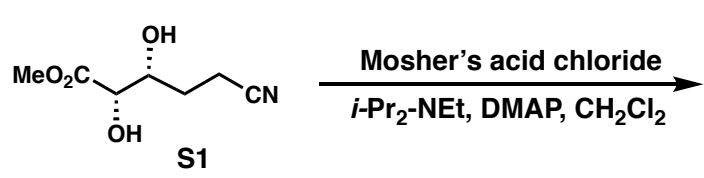
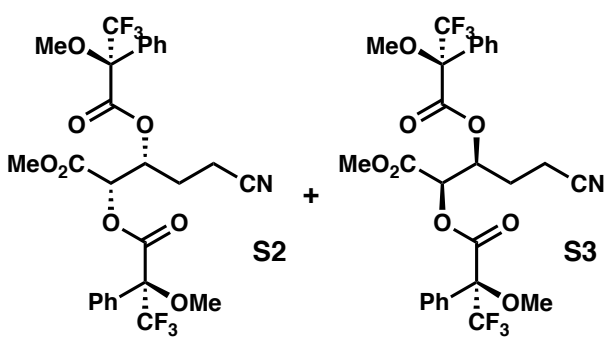

The (S)-Mosher diesters $\mathbf{S} 2$ and $\mathbf{S} 3$ were prepared from $\mathbf{S} 1$ or rac-S1 and $(R)-(-)$-amethoxy-a- trifluoromethylphenylacetyl chloride $((R)-(-)-\mathrm{MTPA}-\mathrm{Cl})$ as follows:

To a flame-dried 1-dram vial was added rac-S1 (10 mg, $0.058 \mathrm{mmol}, 1.0$ equiv.) and $\mathrm{CH}_{2} \mathrm{Cl}_{2}(1.0 \mathrm{~mL})$ under Ar. To this solution was added $i-\mathrm{Pr}_{2} \mathrm{NEt}(50 \mu \mathrm{L}, 0.290 \mathrm{mmol}, 5$ 
equiv.) and one crystal of DMAP. Finally, $(R)-(-)$-MTPA-CI ( $27 \mu \mathrm{L}, 0.144 \mathrm{mmol}, 2.5$ equiv.) was added slowly to the above homogeneous mixture. The vial was capped and the solution allowed to react for 18 hours at room temperature. Upon completion, the reaction was quenched by dilution with $\mathrm{CH}_{2} \mathrm{Cl}_{2}(10 \mathrm{~mL})$ and washed with saturated aqueous sodium bicarbonate $(10 \mathrm{~mL})$. The organic extracts were dried over $\mathrm{MgSO}_{4}$, filtered, and concentrated in vacuo. The resulting crude residue was purified by column chromatography $\left(\mathrm{SiO}_{2}, 3: 1\right.$ hexanes:EtOAc) to afford a 1:1 mixture of bis-(S)-MTPA esters S2 and S3 as a colorless oil ( $25.8 \mathrm{mg}, 73 \%$ yield).

The Mosher esterification reaction above was performed on enantiomerically enriched diol $\mathbf{S} 1$ made by Sharpless asymmetric dihydroxylation to provide predominantly the bis(S)-MTPA ester S2 (derived from (2S,3R)-S1). Integration of the doublets at $5.33 \mathrm{ppm}$ and $5.28 \mathrm{ppm}$ in the ${ }^{1} \mathrm{H}$ NMR spectrum of the product mixture indicated that the ee of $\mathbf{S 1}$ was $\sim 94 \%$ (Figure S1). ${ }^{3}$

Figure S1. Comparison of (S)-MTPA esters of rac-S1 and (S)-MTPA esters of S1 derived from Sharpless dihydroxylation.

\section{Bis-(S)-MTPA ester of rac-S1}
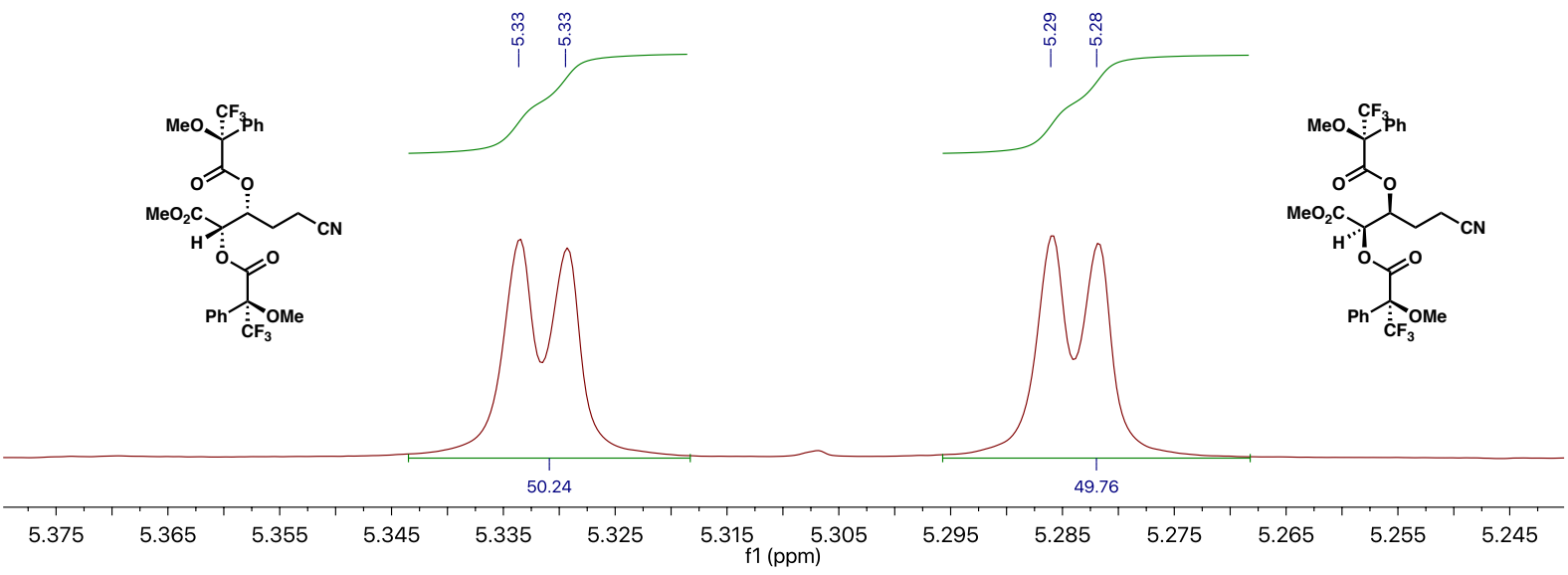

Bis-(S)-MTPA ester of S1 ( 94\% ee)

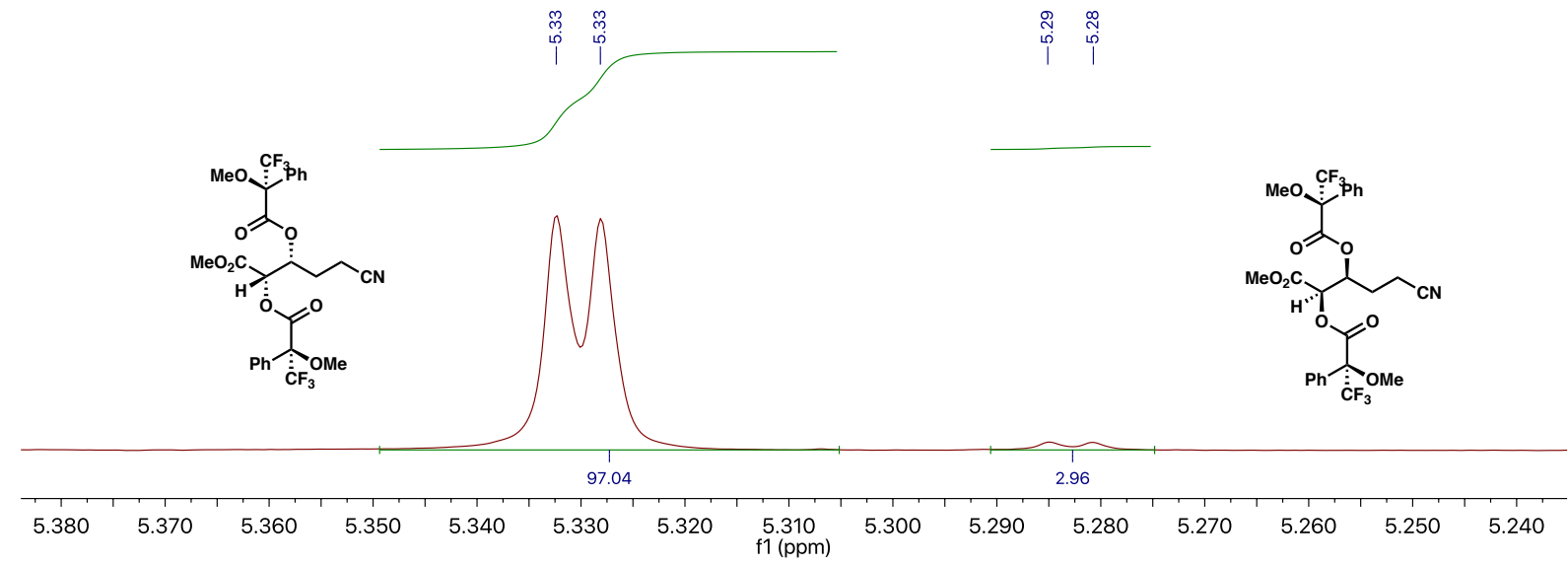


Bis-(S)-MTPA ester (2S,3R)-S2: ${ }^{1} \mathrm{H}$ NMR $\left(500 \mathrm{MHz}, \mathrm{CDCl}_{3}\right) \delta 7.65(\mathrm{~d}, \mathrm{~J}=7.3 \mathrm{~Hz}, 2 \mathrm{H})$, $7.49(\mathrm{~d}, \mathrm{~J}=7.1 \mathrm{~Hz}, 2 \mathrm{H}), 7.46-7.39(\mathrm{~m}, 6 \mathrm{H}$, overlapping signals) 5.62 (ddd, $\mathrm{J}=8.4,5.1$, $2.1 \mathrm{~Hz}, 1 \mathrm{H}), 5.33(\mathrm{~d}, \mathrm{~J}=2.1 \mathrm{~Hz}, 1 \mathrm{H}), 3.71(\mathrm{~s}, 3 \mathrm{H}), 3.61(\mathrm{~s}, 3 \mathrm{H}), 3.38(\mathrm{~s}, 3 \mathrm{H}), 2.21-2.17$ $(\mathrm{m}, 2 \mathrm{H}), 2.00-1.91(\mathrm{~m}, 1 \mathrm{H}), 1.86(\mathrm{dtd}, \mathrm{J}=14.4,8.0,5.1 \mathrm{~Hz}, 1 \mathrm{H})$.

${ }^{13} \mathrm{C}$ NMR $\left(151 \mathrm{MHz}, \mathrm{CDCl}_{3}\right) \delta 166.0,165.9,165.9,131.6,131.0,130.2,130.2,128.9$, 128.7, 127.7, 127.5, $123.3\left(\mathrm{q},{ }^{1} \mathrm{~J}_{\mathrm{CF}}=288.6 \mathrm{~Hz}\right), 123.1$ (q, $\left.{ }^{1} \mathrm{~J}_{\mathrm{CF}}=288.5 \mathrm{~Hz}\right), 117.8,84.9$ $\left(q,{ }^{2} \mathrm{~J}_{\mathrm{CF}}=28.1 \mathrm{~Hz}\right), 84.7\left(\mathrm{q},{ }^{2} \mathrm{~J}_{\mathrm{CF}}=28.2 \mathrm{~Hz}\right), 73.2,72.6,55.9,55.4,53.3,26.7,13.5$.

${ }^{19} \mathrm{~F}$ NMR $\left(656 \mathrm{MHz}, \mathrm{CDCl}_{3}\right): \delta-71.53,-71.77$.

HRMS (ES+) $m / z$ calc'd for $\mathrm{C}_{27} \mathrm{H}_{25} \mathrm{~F}_{6} \mathrm{NO}_{8}[\mathrm{M}+\mathrm{Na}]^{+}:$628.1382, found 628.1362 .

TLC: $\mathrm{R}_{f}=0.21$ (3:1 hexanes:EtOAc, visualized by $\mathrm{KMnO}_{4}$ ).
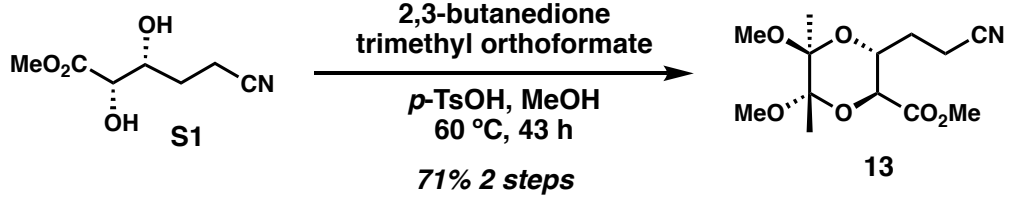

To a $20 \mathrm{~mL}$ scintillation vial charged with crude diol $\mathbf{S 1}(910 \mathrm{mg})$ was added 2,3butanedione $(0.34 \mathrm{~mL}, 3.95 \mathrm{mmol})$, trimethyl orthoformate $(1.96 \mathrm{~mL}, 17.95 \mathrm{mmol})$, and $\mathrm{MeOH}(4.5 \mathrm{~mL}, 0.8 \mathrm{M})$ open to air. $p-\mathrm{TsOH} \cdot \mathrm{H}_{2} \mathrm{O}(34.1 \mathrm{mg}, 0.179 \mathrm{mmol})$ was added in one portion, the vial was capped and submerged in a preheated $60^{\circ} \mathrm{C}$ oil bath. After 19 hours, the reaction mixture was cooled to room temperature and another portion of $p$ $\mathrm{TsOH} \cdot \mathrm{H}_{2} \mathrm{O}$ (34.1 mg, $\left.0.179 \mathrm{mmol}\right)$ was added to the reaction vial which was reheated to $60{ }^{\circ} \mathrm{C}$. After 24 hours, the reaction mixture was cooled to room temperature and $\mathrm{K}_{2} \mathrm{CO}_{3}$ (ca. $150 \mathrm{mg}$ ) was added and stirred vigorously for 5 minutes. The solution was diluted with $\mathrm{CH}_{2} \mathrm{Cl}_{2}(15 \mathrm{~mL})$, filtered, and concentrated in vacuo. The resulting crude residue was purified by column chromatography $\left(\mathrm{SiO}_{2}, 3: 1\right.$ hexanes:EtOAc) to afford $\mathbf{1 3}$ as a pale orange viscous oil $(735 \mathrm{mg}, 71 \%$ yield over two steps).

${ }^{1} \mathrm{H}$ NMR $\left(600 \mathrm{MHz}, \mathrm{CDCl}_{3}\right) \delta 4.17(\mathrm{~d}, \mathrm{~J}=9.8 \mathrm{~Hz}, 1 \mathrm{H}), 4.01$ (td, J = 10.1, $\left.2.8 \mathrm{~Hz}, 1 \mathrm{H}\right), 3.80$ (s, 3H), 3.31 (s, 6H), 2.56 (ddd, J = 7.8, 6.3, $2.8 \mathrm{~Hz}, 2 \mathrm{H}$ ), 1.99 (dtd, J = 14.0, 8.2, $2.8 \mathrm{~Hz}$, $1 \mathrm{H}), 1.83$ (dddd, $\mathrm{J}=14.0,10.3,7.0,5.6 \mathrm{~Hz}, 1 \mathrm{H}), 1.37(\mathrm{~s}, 3 \mathrm{H}), 1.32(\mathrm{~s}, 3 \mathrm{H})$.

${ }^{13} \mathrm{C}$ NMR $\left(151 \mathrm{MHz}, \mathrm{CDCl}_{3}\right) \delta 169.0,119.3,99.4,99.0,72.0,66.6,52.7,48.6,48.5,26.9$, 17.7, 17.5, 13.0.

HRMS (ES+) $\mathrm{m} / \mathrm{z}$ calc'd for $\mathrm{C}_{13} \mathrm{H}_{21} \mathrm{NO}_{6}[\mathrm{M}+\mathrm{Na}]^{+}: 310.1266$, found 310.1266 . $[\alpha]^{22.5} \mathrm{D}+141.1\left(\mathrm{c}=1.01, \mathrm{CHCl}_{3}\right)$.

TLC: $R_{f}=0.22$ (3:1 hexanes:EtOAc, visualized by CAM). 
<smiles>COC(=O)C1O[C@](C)(OC)[C@@](C)(OC)OC1CCC#N</smiles>

13

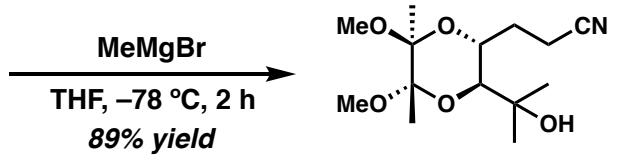

S4

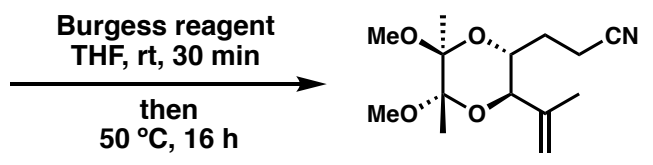

14

To a cooled $\left(-78{ }^{\circ} \mathrm{C}\right)$ solution of $13(1.00 \mathrm{~g}, 3.48 \mathrm{mmol}, 1.0$ equiv.) in THF (17 mL) was added dropwise $\mathrm{MeMgBr}(6.96 \mathrm{~mL}, 20.9 \mathrm{mmol}, 3.0 \mathrm{M}$ in Et2O, 6.0 equiv.) over 5 minutes. Note: an excess of Grignard is necessary for obtaining high yields of S4. After 2 hours at $-78^{\circ} \mathrm{C}$, TLC indicated complete consumption of the starting material and the reaction was quenched slowly with $\mathrm{AcOH}(1.5 \mathrm{~mL})$. The cooling bath was removed and the mixture was diluted with EtOAc $(20 \mathrm{~mL})$ and saturated aqueous $\mathrm{NH}_{4} \mathrm{Cl}(20 \mathrm{~mL})$. The biphasic solution was transferred to a separatory funnel and the layers were separated. The aqueous layer was extracted with EtOAc $(2 \times 10 \mathrm{~mL})$ and the combined organic extracts were dried over $\mathrm{MgSO}_{4}$, filtered, and concentrated in vacuo. The resulting crude residue was purified by column chromatography $\left(\mathrm{SiO}_{2}, 7: 3\right.$ then 1:1 hexanes:EtOAc) to afford $\mathbf{S} 4$ as a pale yellow viscous oil ( $890 \mathrm{mg}, 89 \%$ yield).

${ }^{1} \mathrm{H}$ NMR $\left(600 \mathrm{MHz}, \mathrm{CDCl}_{3}\right) \delta 3.88(\mathrm{t}, \mathrm{J}=9.9 \mathrm{~Hz}, 1 \mathrm{H}), 3.35(\mathrm{~d}, \mathrm{~J}=9.5 \mathrm{~Hz}, 1 \mathrm{H}), 3.28(\mathrm{~s}$, $3 \mathrm{H}$ ), $3.26(\mathrm{~s}, 3 \mathrm{H}), 2.57$ (dtd, J = 22.8, 16.7, 7.0 Hz, 2H), 2.23 (dt, J = 14.7, 7.8 Hz, $1 \mathrm{H}$ ), 1.91 (br s, 1H), 1.81 (ddd, J = 12.2, 8.7, $5.6 \mathrm{~Hz}, 1 \mathrm{H}$ ), 1.29 (s, 6H), 1.28 (s, 3H), 1.26 (s, $3 \mathrm{H})$.

${ }^{13} \mathrm{C}$ NMR $\left(150 \mathrm{MHz}, \mathrm{CDCl}_{3}\right) \delta 120.0,99.0,98.6,76.7,71.7,66.9,48.4,48.2,28.1,27.9$, 25.4, 17.6, 17.6, 13.0.

HRMS (ES+) $\mathrm{m} / \mathrm{z}$ calc'd for $\mathrm{C}_{14} \mathrm{H}_{25} \mathrm{NO}_{5}[\mathrm{M}+\mathrm{Na}]^{+}: 310.1631$, found 310.1626 . $[\alpha]^{21.5} \mathrm{D}-159.8\left(\mathrm{c}=1.00, \mathrm{CHCl}_{3}\right)$.

TLC: $\mathrm{R}_{f}=0.10$ ( $1: 1$ hexanes:Et $2 \mathrm{O}$, visualized by $\left.\mathrm{CAM}\right)$.

NOTE: the data reported for this compound were collected on ent-S4 (derived from Sharpless $A D$ with (DHQ) 2 PHAL as ligand) including the optical rotation!

To a flame-dried $25 \mathrm{~mL}$ round-bottomed flask was added Burgess reagent $(759 \mathrm{mg}, 3.18$ mmol, 1.5 equiv.) followed by THF $(5.0 \mathrm{~mL})$. This flask was placed in a room temperature water bath before slow addition of $\mathbf{S 4}(610 \mathrm{mg}, 2.12 \mathrm{mmol}, 1.0$ equiv) in THF (5.0 mL). After 30 minutes at room temperature, TLC analysis indicated consumption of the starting material and the presence of a more polar compound. The flask was submerged in a preheated $50{ }^{\circ} \mathrm{C}$ oil bath and allowed to react at this temperature overnight. After 16 hours, the flask was cooled to room temperature, quenched with saturated aqueous $\mathrm{NaHCO}_{3}(10 \mathrm{~mL})$ and diluted with EtOAc $(10 \mathrm{~mL})$. The layers were separated and the aqueous phase was extracted with EtOAc $(2 \times 10 \mathrm{~mL})$. The combined organic extracts were dried over $\mathrm{MgSO}_{4}$ and concentrated in vacuo. The crude solids were dry loaded onto approximately $2 \mathrm{~g}$ of $\mathrm{SiO}_{2}$ and purified by column chromatography $\left(\mathrm{SiO}_{2}, 7: 1\right.$ then 4:1 hexanes:EtOAc) to afford 14 as a colorless crystalline solid (444 $\mathrm{mg}, 78 \%$ yield). 
${ }^{1} \mathrm{H}$ NMR $\left(500 \mathrm{MHz}, \mathrm{CDCl}_{3}\right) \delta 5.02-4.99(\mathrm{~m}, 2 \mathrm{H}), 3.92(\mathrm{~d}, \mathrm{~J}=9.7 \mathrm{~Hz}, 1 \mathrm{H}), 3.87(\mathrm{td}, \mathrm{J}=$ 10.0, $2.8 \mathrm{~Hz}, 1 \mathrm{H}), 3.30(\mathrm{~s}, 3 \mathrm{H}), 3.26(\mathrm{~s}, 3 \mathrm{H}), 2.66-2.45(\mathrm{~m}, 2 \mathrm{H}), 1.80(\mathrm{~d}, \mathrm{~J}=1.3 \mathrm{~Hz}, 3 \mathrm{H})$, $1.78-1.71(\mathrm{~m}, 1 \mathrm{H}), 1.70-1.62(\mathrm{~m}, 1 \mathrm{H}), 1.31(\mathrm{~s}, 3 \mathrm{H}), 1.30(\mathrm{~s}, 3 \mathrm{H})$.

${ }^{13} \mathrm{C}$ NMR $\left(126 \mathrm{MHz}, \mathrm{CDCl}_{3}\right) \delta 141.6,119.6,116.7,99.1,99.0,76.6,66.9,48.3,48.1,26.9$, $18.1,17.8,17.8,13.1$.

HRMS (ES+) $\mathrm{m} / \mathrm{z}$ calc'd for $\mathrm{C}_{14} \mathrm{H}_{23} \mathrm{NO}_{4}[\mathrm{M}+\mathrm{Na}]^{+}:$292.1525, found 292.1523 . $[\alpha]^{22.8} \mathrm{D}+195.0\left(\mathrm{c}=1.01, \mathrm{CHCl}_{3}\right)$.

TLC: $\mathrm{R}_{f}=0.48\left(1: 1\right.$ hexanes: $\mathrm{Et}_{2} \mathrm{O}$, visualized by $\left.\mathrm{CAM}\right)$.

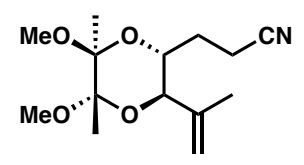

14

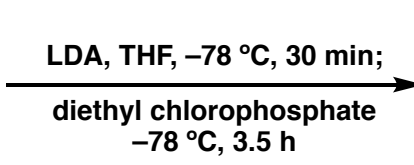

$84 \%$ yield

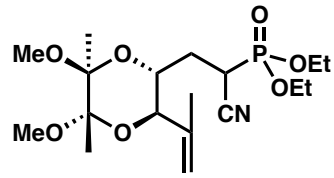

15

To a solution of $i-\mathrm{Pr}_{2} \mathrm{NH}$ (508 mL, $3.63 \mathrm{mmol}, 2.2$ equiv) in THF $(4.0 \mathrm{~mL})$ at $-78^{\circ} \mathrm{C}$ was added dropwise $n$-BuLi (1.38 mL, $2.50 \mathrm{M}, 3.46 \mathrm{mmol}, 2.1$ equiv.). This solution was allowed to warm to $0{ }^{\circ} \mathrm{C}$ and maintained at this temperature for 30 minutes before cooling to $-78{ }^{\circ} \mathrm{C}$. After 5 minutes, a solution of $14(444 \mathrm{mg}, 1.65 \mathrm{mmol}, 1.0$ equiv.) in THF (4.0 $\mathrm{mL}$ ) was added dropwise over 5 minutes (with a $0.2 \mathrm{~mL}$ THF rinse) to the LDA solution. After 30 minutes, diethyl chlorophosphate (286 $\mu \mathrm{L}, 1.98 \mathrm{mmol}, 1.2$ equiv.) was added neat dropwise over 5 minutes. After 3.5 hours, the mixture was quenched at $-78^{\circ} \mathrm{C}$ with sat. aq. $\mathrm{NH}_{4} \mathrm{Cl}(15 \mathrm{~mL})$ and diluted with EtOAc $(15 \mathrm{~mL})$. The layers were separated and the aqueous phase was extracted with EtOAc $(2 \times 15 \mathrm{~mL})$. The combined organic extracts were dried over $\mathrm{MgSO}_{4}$ and concentrated in vacuo. The crude residue was dry loaded onto approximately $1 \mathrm{~g}$ of $\mathrm{SiO}_{2}$ and purified by column chromatography $\left(\mathrm{SiO}_{2}, 1: 1\right.$ then 1:4 hexanes:EtOAc) to afford 15 as a pale yellow viscous oil (561 mg, $84 \%$ yield) and (surprisingly!) as an inconsequential 10:1 mixture of diastereomers. Note: signals are reported for the major diastereomer only.

${ }^{1} \mathrm{H}$ NMR $\left(500 \mathrm{MHz}, \mathrm{CDCl}_{3}\right) \delta 5.02(\mathrm{~s}, 1 \mathrm{H}), 5.01(\mathrm{~s}, 1 \mathrm{H}), 4.24$ (ddq, J = 12.5, 8.7, 7.1 Hz, 4H), $4.06-3.99(\mathrm{~m}, 1 \mathrm{H}), 3.92(\mathrm{~d}, \mathrm{~J}=9.6 \mathrm{~Hz}, 1 \mathrm{H}), 3.44-3.35(\mathrm{~m}, 1 \mathrm{H}), 3.33(\mathrm{~s}, 3 \mathrm{H}), 3.26$ (s, 3H), $1.90-1.84(\mathrm{~m}, 2 \mathrm{H}), 1.81(\mathrm{~s}, 3 \mathrm{H}), 1.38(\mathrm{t}, \mathrm{J}=7.1 \mathrm{~Hz}, 6 \mathrm{H}), 1.30(\mathrm{~s}, 3 \mathrm{H}), 1.29(\mathrm{~s}$, $3 \mathrm{H})$.

${ }^{13} \mathrm{C}$ NMR $\left(126 \mathrm{MHz}, \mathrm{CDCl}_{3}\right) \delta 141.3,117.0,116.1(\mathrm{~d}, \mathrm{~J}=9.2 \mathrm{~Hz}), 99.1(2 \mathrm{C}), 76.6,65.8$, 65.7, $64.3(\mathrm{~d}, \mathrm{~J}=6.8 \mathrm{~Hz}), 63.9(\mathrm{~d}, \mathrm{~J}=6.9 \mathrm{~Hz}), 48.6,48.1,28.6(\mathrm{~d}, \mathrm{~J}=3.6 \mathrm{~Hz}), 26.0(\mathrm{~d}, \mathrm{~J}$ $=146.1 \mathrm{~Hz}), 18.0,17.8(2 \mathrm{C}), 16.5(\mathrm{~d}, \mathrm{~J}=5.8 \mathrm{~Hz})$.

Note that these carbon assignments are tentative and they are assigned to the best of our abilities given the ${ }^{31} \mathrm{P}$ splitting and that this compound is a mixture of diastereomers. HRMS (ES+) $\mathrm{m} / \mathrm{z}$ calc'd for $\mathrm{C}_{18} \mathrm{H}_{32} \mathrm{NO}_{7} \mathrm{P}[\mathrm{M}+\mathrm{Na}]^{+}:$428.1814, found 428.1823 . $[\alpha]^{22.8} \mathrm{D}+90.4\left(\mathrm{c}=1.00, \mathrm{CHCl}_{3}\right)$.

TLC: $\mathrm{R}_{f}=0.20$ (1:1 hexanes:EtOAc, visualized by $\left.\mathrm{KMnO}_{4}\right)$. 
NOTE: both ${ }^{1} \mathrm{H}$ and ${ }^{13} \mathrm{C}$ NMR data reported for this compound were obtained on ent-15 (derived from Sharpless $A D$ with $(D H Q)_{2} P H A L$ as ligand). The optical rotation was measured on 15 as shown in the above scheme.

\section{Cyclization study:}

Note that this cyclization study was performed with material (ent-15) derived from Sharpless $A D$ with $(D H Q)_{2} P H A L$ as ligand.

\section{Synthesis of cyclization substrates:}<smiles>C=C1O[C@](C)(OC)C(C)(OC)OC1CC(C)P(=O)(OCC)OCC</smiles><smiles>COc1cc(CCC=O)cc(OC)c1</smiles>

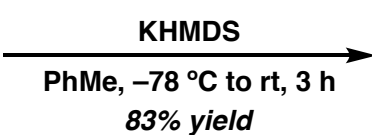

$83 \%$ yield

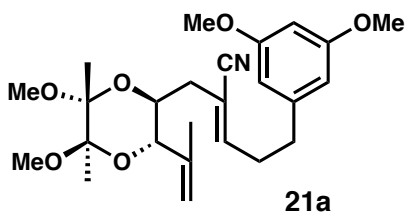

To a solution of cyanophosphonate (ent-15) $(248 \mathrm{mg}, 0.611 \mathrm{mmol}, 1.0$ equiv.; azeotroped from PhMe $2 \times 3 \mathrm{~mL}$ prior to use) in dry PhMe $(4.1 \mathrm{~mL})$ at $-78{ }^{\circ} \mathrm{C}$ was added KHMDS (1.22 mL, $0.5 \mathrm{M}$ in PhMe, $0.611 \mathrm{mmol}, 1.0$ equiv.) dropwise and the mixture was stirred at this temperature for 30 minutes. A solution of $\mathbf{S 5}^{4}(131 \mathrm{mg}, 0.673 \mathrm{mmol}, 1.1$ equiv.; azeotroped from PhMe $2 \times 3 \mathrm{~mL}$ prior to use) in PhMe $(2.0 \mathrm{~mL})$ was then added dropwise slowly at $-78^{\circ} \mathrm{C}$ to the cyanophosphonate/KHMDS mixture. After 1.5 hours at $-78{ }^{\circ} \mathrm{C}$, the cooling bath was removed and the reaction was allowed to warm to room temperature over an hour. TLC analysis indicated consumption of the phosphonate and formation of a less polar, UV active compound. The contents of the reaction flask were poured into a separatory funnel containing sat. aq. $\mathrm{NH}_{4} \mathrm{Cl}(15 \mathrm{~mL})$ and the mixture was extracted with EtOAc $(3 \times 10 \mathrm{~mL})$. The combined organic extracts were dried over $\mathrm{MgSO}_{4}$, filtered, and concentrated in vacuo. The crude residue was purified by column chromatography $\left(\mathrm{SiO}_{2}\right.$, $5: 1$ hexanes:EtOAc) to afford $\mathbf{2 1 a}(225 \mathrm{mg}, 83 \%$ yield, $\sim 10: 1 \mathrm{Z:E})$ as a colorless viscous oil.

${ }^{1} \mathrm{H}$ NMR $\left(500 \mathrm{MHz}, \mathrm{CDCl}_{3}\right) \delta 6.35(\mathrm{~d}, \mathrm{~J}=2.2 \mathrm{~Hz}, 1 \mathrm{H}), 6.32(\mathrm{~d}, \mathrm{~J}=2.4 \mathrm{~Hz}, 1 \mathrm{H}), 6.26(\mathrm{t}, \mathrm{J}$ $=7.0 \mathrm{~Hz}, 1 \mathrm{H}), 5.00(\mathrm{~s}, 2 \mathrm{H}), 3.99-3.87(\mathrm{~m}, 2 \mathrm{H}$, overlapping signals $), 3.79(\mathrm{~s}, 3 \mathrm{H}), 3.26$ (s, 3H), $3.21(\mathrm{~s}, 3 \mathrm{H}), 2.78-2.59(\mathrm{~m}, 2 \mathrm{H}), 2.26(\mathrm{~d}, \mathrm{~J}=14.4 \mathrm{~Hz}, 1 \mathrm{H}), 2.18(\mathrm{dd}, \mathrm{J}=14.4$, $9.2 \mathrm{~Hz}, 1 \mathrm{H}), 1.80(\mathrm{~s}, 3 \mathrm{H}), 1.28(\mathrm{~s}, 3 \mathrm{H}), 1.26(\mathrm{~s}, 3 \mathrm{H})$.

${ }^{13} \mathrm{C}$ NMR $\left(126 \mathrm{MHz}, \mathrm{CDCl}_{3}\right) \delta 161.0,149.4,142.6,141.8,117.5,116.8,111.6,106.5$, 99.1, 99.0, 98.4, 76.5, 67.2, 55.4, 48.2, 48.0, 35.9, 35.1, 32.8, 18.1, 17.8, 17.7 .

HRMS (ES+) $\mathrm{m} / \mathrm{z}$ calc'd for $\mathrm{C}_{25} \mathrm{H}_{35} \mathrm{NO}_{6}[\mathrm{M}+\mathrm{Na}]^{+}: 468.2362$, found 468.2359 .

TLC: $\mathrm{R}_{f}=0.50$ (2:1 hexanes:EtOAc, visualized by $\mathrm{KMnO}_{4}$ ). 


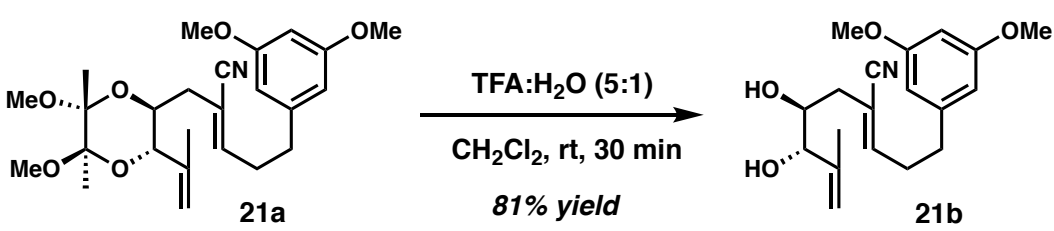

To a solution of polyene $21 \mathrm{a}$ (120 mg, $0.269 \mathrm{mmol}, 1.0$ equiv.) in $\mathrm{CH}_{2} \mathrm{Cl}_{2}(5.4 \mathrm{~mL}$ ) at room temperature and open to air was added a mixture of TFA: $\mathrm{H}_{2} \mathrm{O}(5: 1,400 \mu \mathrm{L})$. After 20 minutes, a dark blue solution formed, and the reaction was stopped by adding sat. aq. $\mathrm{NaHCO}_{3}(10 \mathrm{~mL})$. The biphasic mixture was transferred to a separatory funnel, the layers were separated and the aqueous phase was extracted with $\mathrm{CH}_{2} \mathrm{Cl}_{2}(2 \times 10 \mathrm{~mL})$. The combined organic extracts were dried over $\mathrm{MgSO}_{4}$, filtered, and concentrated in vacuo. The resulting crude residue was purified by column chromatography $\left(\mathrm{SiO}_{2}, 2: 1\right.$ then $2: 3$ hexanes:EtOAc) to afford 21b as a colorless viscous oil ( $72.8 \mathrm{mg}, 81 \%$ yield).

${ }^{1} \mathrm{H}$ NMR $\left(500 \mathrm{MHz}, \mathrm{CDCl}_{3}\right) \delta 6.35(\mathrm{~d}, \mathrm{~J}=2.3 \mathrm{~Hz}, 1 \mathrm{H}), 6.32(\mathrm{~d}, \mathrm{~J}=2.3 \mathrm{~Hz}, 1 \mathrm{H}), 6.26(\mathrm{t}, \mathrm{J}$ $=7.0 \mathrm{~Hz}, 1 \mathrm{H}), 5.04(\mathrm{~s}, 1 \mathrm{H}), 5.01(\mathrm{~s}, 1 \mathrm{H}), 3.81-3.79(\mathrm{~m}, 7 \mathrm{H}$, overlapping signals $), 3.75$ $(\mathrm{d}, \mathrm{J}=4.9 \mathrm{~Hz}, 1 \mathrm{H}), 2.74(\mathrm{~m}, 4 \mathrm{H}), 2.41(\mathrm{dd}, \mathrm{J}=14.3,4.8 \mathrm{~Hz}, 1 \mathrm{H}), 2.34(\mathrm{dd}, \mathrm{J}=14.5,8.1$ $\mathrm{Hz}, 1 \mathrm{H}), 2.16$ (brs, 2H), $1.74(\mathrm{~s}, 3 \mathrm{H})$.

${ }^{13} \mathrm{C}$ NMR $\left(126 \mathrm{MHz}, \mathrm{CDCl}_{3}\right) \delta 161.0,149.6,144.5,142.6,117.5,113.8,112.0,106.8$, 106.7, 98.4, 70.0, 55.5, 38.2, 34.9, 33.0, 18.5 .

HRMS (ES+) $\mathrm{m} / \mathrm{z}$ calc'd for $\mathrm{C}_{19} \mathrm{H}_{25} \mathrm{NO}_{4}[\mathrm{M}+\mathrm{Na}]^{+}: 354.1681$, found 354.1671 .

TLC: $R_{f}=0.44$ (1:1 hexanes:EtOAc, visualized by CAM).
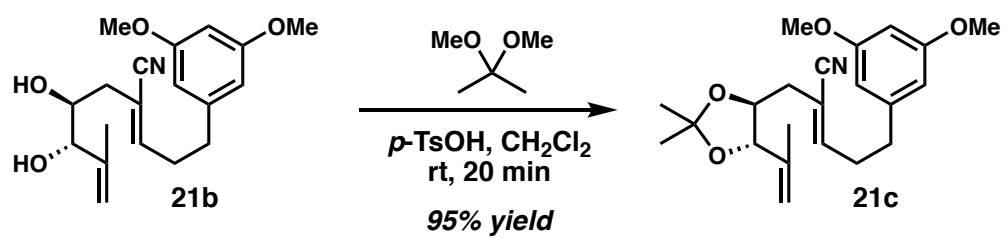

To a $20 \mathrm{~mL}$ vial containing 21b (72.8 $\mathrm{mg}, 0.219 \mathrm{mmol}, 1.0$ equiv) was added 2,2dimethoxypropane $\left(0.27 \mathrm{~mL}, 2.19 \mathrm{mmol}, 10.0\right.$ equiv.) and $\mathrm{CH}_{2} \mathrm{Cl}_{2}(2.2 \mathrm{~mL})$ open to air. Next, $p$-TsOH monohydrate $(4.1 \mathrm{mg}, 0.022 \mathrm{mmol}, 0.1$ equiv.) was added in one portion, the vial was capped and allowed to react at room temperature. After 20 minutes, TLC indicated consumption of the diol and formation of a new, less polar compound. The reaction mixture was diluted with EtOAc $(20 \mathrm{~mL})$ and transferred to a separatory funnel. The mixture was washed with sat. aq. $\mathrm{NaHCO}_{3}(2 \times 10 \mathrm{~mL})$, dried over $\mathrm{MgSO}_{4}$, and concentrated in vacuo. The crude residue was purified by column chromatography $\left(\mathrm{SiO}_{2}\right.$, 5:1 hexanes:EtOAc) to afford $\mathbf{2 1 c}$ as a colorless oil $(79.1 \mathrm{mg}, 95 \%$ yield).

${ }^{1} \mathrm{H}$ NMR $\left(600 \mathrm{MHz}, \mathrm{CDCl}_{3}\right) \delta 6.35(\mathrm{~d}, \mathrm{~J}=2.2 \mathrm{~Hz}, 1 \mathrm{H}), 6.33(\mathrm{~d}, \mathrm{~J}=2.5 \mathrm{~Hz}, 1 \mathrm{H}), 6.27(\mathrm{t}, \mathrm{J}$ $=7.5 \mathrm{~Hz}, 1 \mathrm{H}), 5.02(\mathrm{~s}, 1 \mathrm{H}), 5.00(\mathrm{~s}, 1 \mathrm{H}), 4.05(\mathrm{~d}, \mathrm{~J}=8.4 \mathrm{~Hz}, 1 \mathrm{H}), 3.92(\mathrm{td}, \mathrm{J}=8.1,3.6$ $\mathrm{Hz}, 1 \mathrm{H}), 3.79(\mathrm{~s}, 3 \mathrm{H}), 2.72(\mathrm{~m}, 4 \mathrm{H}), 2.47(\mathrm{dd}, \mathrm{J}=14.9,3.6 \mathrm{~Hz}, 1 \mathrm{H}), 2.38(\mathrm{dd}, \mathrm{J}=14.8,7.7$ $\mathrm{Hz}, 1 \mathrm{H}), 1.78(\mathrm{~s}, 3 \mathrm{H}), 1.43(\mathrm{~s}, 3 \mathrm{H}), 1.41(\mathrm{~s}, 3 \mathrm{H})$. 
${ }^{13} \mathrm{C}$ NMR $\left(150 \mathrm{MHz}, \mathrm{CDCl}_{3}\right) \delta 161.0,149.5,142.6,141.1,117.5,115.5,111.3,109.3$, 106.6, 98.4, 84.4, 76.4, 55.4, 36.9, 35.0, 32.9, 27.4, 27.0, 17.3.

HRMS (ES+) $m / z$ calc'd for $\mathrm{C}_{22} \mathrm{H}_{29} \mathrm{NO}_{4}[\mathrm{M}+\mathrm{Na}]^{+}: 394.1994$, found 394.1994 .

TLC: $R_{f}=0.21$ (9:1 hexanes:EtOAc, visualized by CAM).

\section{General procedure for cobalt-catalyzed bicyclizations}

A 1-dram vial was charged with a magnetic stirring bar, cyclization substrate (1.0 equiv.), cobalt(II) precatalyst (0.1 equiv.), and 1-fluoro-2,4,6-trimethylpyridinium tetrafluoroborate (3.0 equiv.) open to air. The vial was capped with a rubber septum and the reagents were dissolved in HFIP (ca. $0.1 \mathrm{M}$, an additional $\sim 0.2 \mathrm{~mL}$ was added to account for solvent lost due to sparging; a dark forest green solution forms immediately). A balloon with a syringe needle was used to bubble argon through solution for 10 minutes (a syringe needle was used as an outlet). The vial was sealed from the atmosphere and 1,1,3,3tetramethyldisiloxane (3.0 equiv.) was added dropwise slowly (approximately 1 drop per 3 seconds). The resulting solution gradually changed from dark green to a tan-brown or red color. After 4 hours, the reaction mixture was exposed to the atmosphere and volatiles were removed in vacuo. The crude residue was directly purified by flash column chromatography on silica gel to afford pure cyclization products. Note: if a ${ }^{1} H$ NMR spectrum of the crude reaction mixture is desired, the crude residue should be passed through a short silica plug (using EtOAc as eluent) to remove paramagnetic cobalt complexes.

X-ray quality crystals were grown by slow evaporation from a mixture of hexanes: $\mathrm{CH}_{2} \mathrm{Cl}_{2}$ (ca. 4:1) over several days. The setup for crystallization is as follows: the purified cyclization products were transferred to a 1-dram vial and dissolved in a minimum amount of the solvent mixture. This vial was then placed in a $20 \mathrm{~mL}$ scintillation vial containing hexanes (ca. 5-8 mL), which was capped. Slow diffusion of $\mathrm{CH}_{2} \mathrm{Cl}_{2}$ out of the inner vial over $>24$ hours produced crystals of suitable quality for X-ray diffraction studies.
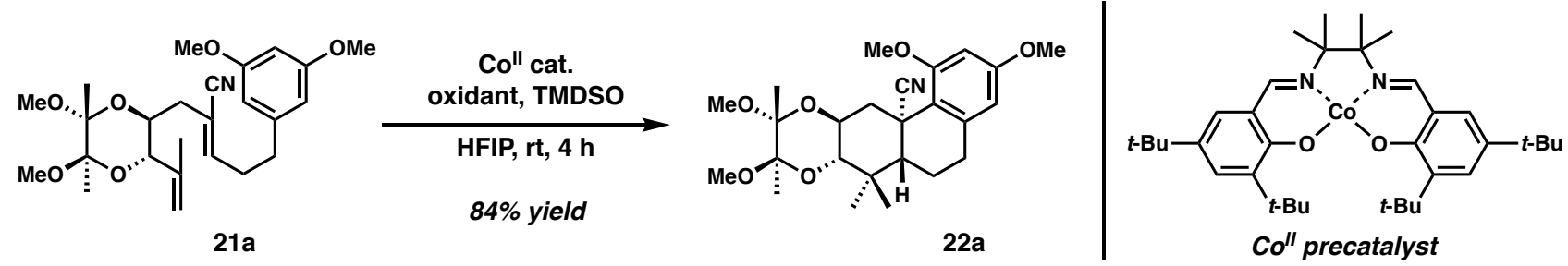

Prepared according to the general procedure using butanedione protected substrate 21a (38.9 $\mathrm{mg}, 0.087 \mathrm{mmol}$ ), cobalt(II) precatalyst $(5.2 \mathrm{mg}, 0.0087 \mathrm{mmol}), 1$-fluoro-2,4,6trimethylpyridinium tetrafluoroborate $(59.4 \mathrm{mg}, 0.262 \mathrm{mmol}), \mathrm{TMDSO}(46 \mu \mathrm{L}, 0.262$ $\mathrm{mmol}$ ), and HFIP (1.1 mL). Yield of 22a: $84 \%$ (32.9 mg, colorless solid); purified on silica gel using 5:1 hexanes:EtOAc.

${ }^{1} \mathrm{H}$ NMR $\left(500 \mathrm{MHz}, \mathrm{CDCl}_{3}\right) \delta 6.32(\mathrm{~d}, \mathrm{~J}=2.6 \mathrm{~Hz}, 1 \mathrm{H}), 6.24(\mathrm{~d}, \mathrm{~J}=2.5 \mathrm{~Hz}, 1 \mathrm{H}), 4.15$ (ddd, $\mathrm{J}=12.3,10.1,4.1 \mathrm{~Hz}, 1 \mathrm{H}), 3.86(\mathrm{~s}, 3 \mathrm{H}), 3.79(\mathrm{~s}, 3 \mathrm{H}), 3.61(\mathrm{dd}, \mathrm{J}=12.9,4.1 \mathrm{~Hz}, 1 \mathrm{H}), 3.35$ 
(s, 3H), $3.26-3.23(\mathrm{~m}, 4 \mathrm{H}$, overlapping signals), $2.93-2.83(\mathrm{~m}, 2 \mathrm{H}), 2.01(\mathrm{dd}, \mathrm{J}=12.1$, $4.4 \mathrm{~Hz}, 1 \mathrm{H}), 1.75(\mathrm{dtd}, \mathrm{J}=13.1,11.5,6.3 \mathrm{~Hz}, 1 \mathrm{H}), 1.43-1.35(\mathrm{~m}, 2 \mathrm{H}), 1.32(\mathrm{~s}, 3 \mathrm{H}), 1.31$ $(\mathrm{s}, 3 \mathrm{H}), 1.21(\mathrm{~s}, 3 \mathrm{H}), 1.13(\mathrm{~s}, 3 \mathrm{H})$.

${ }^{13} \mathrm{C}$ NMR $\left(126 \mathrm{MHz}, \mathrm{CDCl}_{3}\right) \delta 160.1,160.0,139.6,122.4,118.1,105.4,99.8,99.4,97.7$, 77.6, 65.1, 55.8, 55.4, 52.6, 48.2, 47.8, 38.9, 38.3, 37.7, 32.8, 27.5, 20.7, 17.98, 17.97, 15.7.

HRMS (ES+) $\mathrm{m} / \mathrm{z}$ calc'd for $\mathrm{C}_{25} \mathrm{H}_{35} \mathrm{NO}_{6}[\mathrm{M}+\mathrm{Na}]^{+}: 468.2362$, found 468.2383 .

TLC: $\mathrm{R}_{f}=0.31$ (3:1 hexanes:EtOAc, visualized by $\mathrm{KMnO}_{4}$ ).
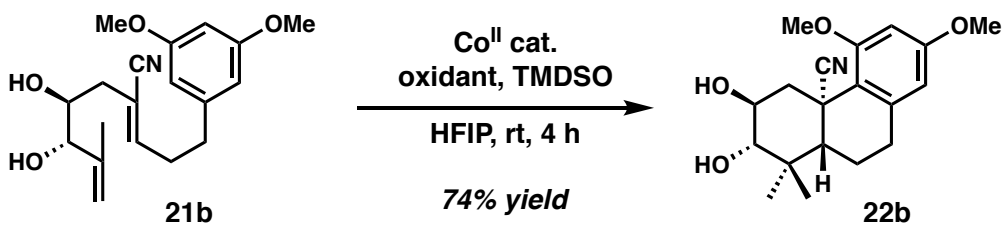

Prepared according to the general procedure using diol $21 \mathrm{~b}(20.7 \mathrm{mg}, 0.062 \mathrm{mmol})$, cobalt(II) precatalyst (3.7 $\mathrm{mg}, 0.0062 \mathrm{mmol}$ ), 1-fluoro-2,4,6-trimethylpyridinium tetrafluoroborate (42.2 $\mathrm{mg}, 0.186 \mathrm{mmol}), \operatorname{TMDSO}(33 \mu \mathrm{L}, 0.186 \mathrm{mmol})$, and HFIP (1.0 $\mathrm{mL}$ ). Yield of 22b: $74 \%$ (15.3 mg, colorless solid); purified on silica gel using 1:1 then 3:7 hexanes:EtOAc.

${ }^{1} \mathrm{H}$ NMR $\left(500 \mathrm{MHz}, \mathrm{CDCl}_{3}\right) \delta 6.33(\mathrm{~d}, \mathrm{~J}=2.5 \mathrm{~Hz}, 1 \mathrm{H}), 6.25$ (d, J = 2.5 Hz, 1H), 4.07 (ddd, $\mathrm{J}=11.7,9.6,4.2 \mathrm{~Hz}, 1 \mathrm{H}), 3.87(\mathrm{~s}, 3 \mathrm{H}), 3.79(\mathrm{~s}, 3 \mathrm{H}), 3.12(\mathrm{~d}, \mathrm{~J}=9.6 \mathrm{~Hz}, 1 \mathrm{H}), 3.00-2.69$ $(\mathrm{m}, 2 \mathrm{H}), 2.25-2.20(\mathrm{~m}, 1 \mathrm{H}), 2.05-1.93(\mathrm{~m}, 1 \mathrm{H}), 1.75(\mathrm{dtd}, \mathrm{J}=13.3,11.5,6.2 \mathrm{~Hz}, 1 \mathrm{H})$, $1.48-1.38(\mathrm{~m}, 1 \mathrm{H}), 1.32(\mathrm{t}, \mathrm{J}=12.5 \mathrm{~Hz}, 1 \mathrm{H}), 1.17(\mathrm{~s}, 3 \mathrm{H}), 1.16(\mathrm{~s}, 3 \mathrm{H})$.

${ }^{13} \mathrm{C}$ NMR $\left(126 \mathrm{MHz}, \mathrm{CDCl}_{3}\right) \delta 160.2,159.9,139.6,122.4,117.9,105.4,97.8,82.5,69.2$, $55.7,55.4,52.3,39.9,39.5,38.6,32.7,28.2,21.0,15.5$.

HRMS (ES+) $\mathrm{m} / \mathrm{z}$ calc'd for $\mathrm{C}_{19} \mathrm{H}_{25} \mathrm{NO}_{4}[\mathrm{M}+\mathrm{Na}]^{+}: 354.1681$, found 354.1695 .

TLC: $\mathrm{R}_{f}=0.36$ (3:2 EtOAc:PhH, visualized by $\mathrm{KMnO}_{4}$ ).
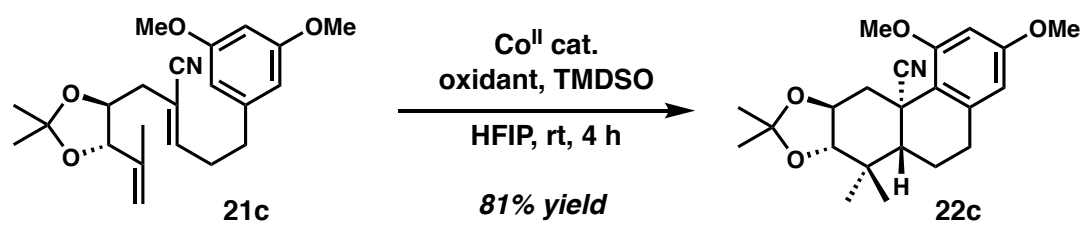

Prepared according to the general procedure using acetonide $21 \mathrm{c}(27.9 \mathrm{mg}, 0.075 \mathrm{mmol})$, cobalt(II) precatalyst $(4.5 \mathrm{mg}, 0.0075 \mathrm{mmol})$, 1-fluoro-2,4,6-trimethylpyridinium tetrafluoroborate $(51.0 \mathrm{mg}, 0.225 \mathrm{mmol})$, TMDSO (40 $\mu \mathrm{L}, 0.225 \mathrm{mmol})$, and HFIP (1.0 $\mathrm{mL}$ ). Yield of 22c: $82 \%$ (22.8 $\mathrm{mg}$, colorless foam); purified on silica gel using 9:1 hexanes:EtOAc.

1'H NMR (500 MHz, CDCl $) \delta 6.34(\mathrm{~d}, \mathrm{~J}=2.6 \mathrm{~Hz}, 1 \mathrm{H}), 6.26(\mathrm{~d}, \mathrm{~J}=2.5 \mathrm{~Hz}, 1 \mathrm{H}), 4.10$ (ddd, $\mathrm{J}=12.0,9.4,3.7 \mathrm{~Hz}, 1 \mathrm{H}), 4.03(\mathrm{dd}, \mathrm{J}=12.3,3.7 \mathrm{~Hz}, 1 \mathrm{H}), 3.85(\mathrm{~s}, 3 \mathrm{H}), 3.79(\mathrm{~s}, 3 \mathrm{H}), 3.16$ 
$(\mathrm{d}, \mathrm{J}=9.4 \mathrm{~Hz}, 1 \mathrm{H}), 2.97-2.84(\mathrm{~m}, 2 \mathrm{H}), 2.04(\mathrm{dd}, \mathrm{J}=13.3,5.0 \mathrm{~Hz}, 1 \mathrm{H}), 1.80(\mathrm{dtd}, \mathrm{J}=$ 13.6, 11.8, $5.9 \mathrm{~Hz}, 1 \mathrm{H}), 1.49(\mathrm{~s}, 3 \mathrm{H}), 1.45(\mathrm{~s}, 3 \mathrm{H}), 1.46-1.39(\mathrm{~m}, 2 \mathrm{H}), 1.22(\mathrm{~s}, 3 \mathrm{H}), 1.18$ (s, 3H).

${ }^{13} \mathrm{C}$ NMR $\left(126 \mathrm{MHz}, \mathrm{CDCl}_{3}\right) \delta 160.3,160.1,139.7,122.3,117.9,109.8,105.6,97.9,87.7$, 72.7, 55.6, 55.4, 53.0, 39.9, 38.0, 36.6, 33.1, 28.3, 27.2, 27.0, 20.3, 15.0.

HRMS (ES+) $\mathrm{m} / \mathrm{z}$ calc'd for $\mathrm{C}_{22} \mathrm{H}_{29} \mathrm{NO}_{4}[\mathrm{M}+\mathrm{H}]^{+}: 372.2175$., found 372.2162 .

TLC: $\mathrm{R}_{f}=0.25$ (5:1 hexanes:EtOAc, visualized by $\mathrm{KMnO}_{4}$ ).

\section{Synthesis of Plebeianiol A:}

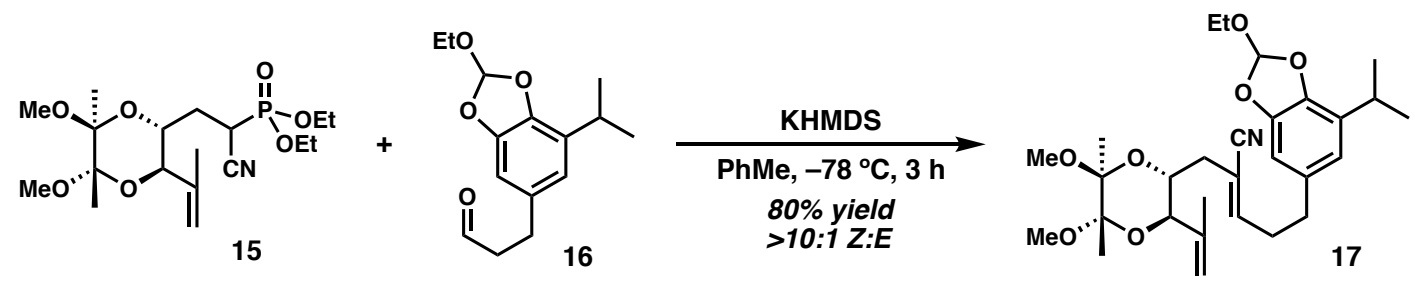

A solution of cyanophosphonate 15 (509 mg, $1.26 \mathrm{mmol}, 1.0$ equiv.) in dry $\mathrm{PhMe}$ (14.0 $\mathrm{mL}$ ) was cooled to $-78^{\circ} \mathrm{C}$. KHMDS (0.5 M in PhMe, $2.5 \mathrm{~mL}, 1.26 \mathrm{mmol}$, 1.0 equiv.), was added dropwise by syringe over 5 minutes. The resulting orange solution was placed in an ice bath to stir at $0{ }^{\circ} \mathrm{C}$ for 5 minutes and then cooled once more to $-78{ }^{\circ} \mathrm{C}$. A solution of $16^{5}$ in dry $\mathrm{PhMe}(3.0 \mathrm{~mL})$ was added dropwise by syringe and the reaction mixture stirred for 3 hours. The reaction was quenched with the addition of saturated aqueous ammonium chloride $(15 \mathrm{~mL})$ and then diluted with deionized water $(5 \mathrm{~mL})$. The biphasic solution was transferred to a separatory funnel and the layers were separated. The aqueous layer was extracted with $\mathrm{Et}_{2} \mathrm{O}(3 \times 15 \mathrm{~mL})$. The combined organic extracts were washed with brine $(15 \mathrm{~mL})$, dried over sodium sulfate, filtered, and concentrated under reduced pressure. The crude residue was purified by flash column chromatography $\left(\mathrm{SiO}_{2}\right.$, 94:6 hexanes:EtOAc), providing diene 17 (518 mg, $1.01 \mathrm{mmol}, 80 \%,>10: 1 \mathrm{Z:E,} \mathrm{\sim 95 \%}$ purity) as a faint yellow oil.

${ }^{1} \mathrm{H}$ NMR $\left(500 \mathrm{MHz}, \mathrm{CDCl}_{3}\right) \delta 6.81(\mathrm{~s}, 1 \mathrm{H}), 6.53(\mathrm{app} \mathrm{d}, J=9.1 \mathrm{~Hz}, 2 \mathrm{H}), 6.27-6.20(\mathrm{~m}$, $1 \mathrm{H}$ ), 4.97 (app d, $J=6.5,2 \mathrm{H}$ ), $3.96-3.86(\mathrm{~m}, 2 \mathrm{H}), 3.67(\mathrm{q}, J=7.1 \mathrm{~Hz}, 2 \mathrm{H}), 3.29-3.14$ $(\mathrm{m}, 6 \mathrm{H}), 2.99(\mathrm{~h}, J=7.0 \mathrm{~Hz}, 1 \mathrm{H}), 2.72-2.54(\mathrm{~m}, 4 \mathrm{H}), 2.23(\mathrm{~d}, J=14.3 \mathrm{~Hz}, 1 \mathrm{H}), 2.16(\mathrm{dd}$, $J=14.4,8.7 \mathrm{~Hz}, 1 \mathrm{H}), 1.78(\mathrm{bs}, 3 \mathrm{H}), 1.32-1.15(\mathrm{~m}, 15 \mathrm{H})$.

${ }^{13} \mathrm{C}$ NMR $\left(151 \mathrm{MHz}, \mathrm{CDCl}_{3}\right) \delta 149.3,149.3,145.8,145.8,141.8,141.6,133.7,133.7$, 129.4, 119.3, 118.6, 117.3, 116.6, 111.3, 105.9, 105.8, 98.9, 98.9, 77.4, 76.3, 67.0, 58.9, 48.0, 48.0, 47.9, 35.8, 34.7, 34.7, 33.4, 33.3, 29.2, 22.2, 22.1, 17.9, 17.6, 17.6, 14.9.

HRMS (ES+) $\mathrm{m} / \mathrm{z}$ calc'd for $\mathrm{C}_{29} \mathrm{H}_{41} \mathrm{NO}_{7}[\mathrm{M}+\mathrm{Na}]^{+}: 538.2781$, found 538.2773.

TLC: $R_{f}=0.20$ (9:1 hexanes:EtOAc, visualized by CAM) 


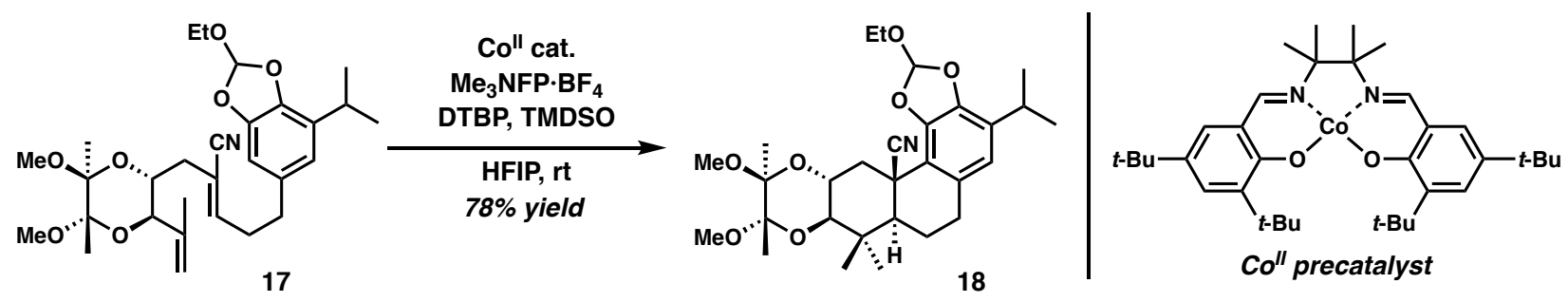

Diene 17 (208 mg, $0.40 \mathrm{mmol}, 1.0$ equiv.), cobalt(II) precatalyst $(24.5 \mathrm{mg}, 0.04 \mathrm{mmol}, 0.1$ equiv.), 1-fluoro-2,4,6-trimethylpyridinium tetrafluoroborate (275 mg, $1.21 \mathrm{mmol}, 3.0$ equiv.), and 2,6-di-tert-butylpyridine $(0.2 \mathrm{~mL}, 0.81 \mathrm{mmol}, 2.0$ equiv.) were placed in a vial open to air and dissolved in HFIP $(9.0 \mathrm{~mL})$. The vial was capped with a rubber septum and sealed with parafilm. The septum was vented with a syringe needle and the solution was sparged under positive pressure with an Ar balloon for 15 minutes. The vent was removed from the septum to leave the solution under Ar and TMDSO $(0.2 \mathrm{~mL}, 1.21 \mathrm{mmol}$, 3.0 equiv.) was added dropwise by syringe over 5 minutes. The reaction mixture was allowed to stir for 4 hours. The mixture was concentrated under reduced pressure. The crude residue was purified by flash column chromatography $\left(\mathrm{SiO}_{2}, 4 \%\right.$ EtOAc in hexanes), providing nitrile 18 (163 $\mathrm{mg}, 0.32 \mathrm{mmol}, 78 \%)$ as a white foam. It was isolated as a mixture of diastereomers at the orthoester stereogenic center. Surprisingly, these isomers could be separated $\left(\mathrm{SiO}_{2}, 98: 2\right.$ then 96:4 hexanes:EtOAc) but assignment of configuration at that center could not be made. To make characterization of subsequent intermediates more facile, initially the more less diastereomer was carried through the rest of the sequence to plebeianiol A. However, the mixture of diastereomers performed identically throughout this sequence.

More polar diastereomer 18a (relative configuration unassigned):

${ }^{1} \mathrm{H}$ NMR $\left(600 \mathrm{MHz}, \mathrm{CDCl}_{3}\right) \delta 6.91(\mathrm{~s}, 1 \mathrm{H}), 6.51(\mathrm{~s}, 1 \mathrm{H}), 4.15$ (ddd, $J=12.2,10.1,4.1 \mathrm{~Hz}$, $1 \mathrm{H}$ ), 3.68 (dq, $J=9.4,7.2 \mathrm{~Hz}, 1 \mathrm{H}$ ), $3.58(\mathrm{dq}, J=9.4,7.1 \mathrm{~Hz}, 1 \mathrm{H}), 3.41$ (dd, $J=13.0,4.1$ $\mathrm{Hz}, 1 \mathrm{H}$ ), $3.35(\mathrm{~s}, 3 \mathrm{H}), 3.26-3.22(\mathrm{~m}, 4 \mathrm{H}), 3.00(\mathrm{~h}, J=7.0 \mathrm{~Hz}, 1 \mathrm{H}), 2.93$ (dd, $J=16.6,4.3$ $\mathrm{Hz}, 1 \mathrm{H}$ ), 2.80 (ddd, $J=17.0,12.6,5.9 \mathrm{~Hz}, 1 \mathrm{H}), 2.07-2.00(\mathrm{~m}, 1 \mathrm{H}), 1.81$ (qd, $J=12.7$, $5.2 \mathrm{~Hz}, 1 \mathrm{H}), 1.63(\mathrm{t}, J=12.6 \mathrm{~Hz}, 1 \mathrm{H}), 1.41(\mathrm{dd}, J=12.0,1.6 \mathrm{~Hz}, 1 \mathrm{H}), 1.31(\mathrm{~s}, 6 \mathrm{H}), 1.26$ $-1.22(\mathrm{~m}, 9 \mathrm{H}), 1.18(\mathrm{~s}, 3 \mathrm{H}), 1.12(\mathrm{~s}, 3 \mathrm{H})$.

${ }^{13} \mathrm{C}$ NMR $\left(151 \mathrm{MHz}, \mathrm{CDCl}_{3}\right) \delta 143.4,142.2,129.9,129.5,121.7,120.3,118.5,116.3$, $99.8,99.4,77.4,64.8,57.9,51.0,48.3,47.8,38.6,38.2,37.4,30.8,28.9,27.1,22.2$, $22.0,21.3,17.9,15.3,15.0$.

HRMS (ES+) $\mathrm{m} / \mathrm{z}$ calc'd for $\mathrm{C}_{29} \mathrm{H}_{41} \mathrm{NO}_{7}[\mathrm{M}+\mathrm{Na}]^{+}: 538.2781$, found 538.2799.

TLC: $R_{f}=0.23$ ( $9: 1$ hexanes:EtOAc, visualized by CAM)

Less polar diastereomer $\mathbf{1 8 b}$ (relative configuration unassigned):

${ }^{1} \mathrm{H}$ NMR $\left(600 \mathrm{MHz}, \mathrm{CDCl}_{3}\right) \delta 6.92(\mathrm{~s}, 1 \mathrm{H}), 6.51$ (s, $\left.1 \mathrm{H}\right), 4.15$ (ddd, $J=12.2,10.1,4.1 \mathrm{~Hz}$, $1 \mathrm{H}), 3.62(\mathrm{qd}, J=7.0,5.1 \mathrm{~Hz}, 2 \mathrm{H}), 3.41(\mathrm{dd}, J=13.0,4.1 \mathrm{~Hz}, 1 \mathrm{H}), 3.34(\mathrm{~s}, 3 \mathrm{H}), 3.26-$ $3.23(\mathrm{~m}, 4 \mathrm{H}), 3.00(\mathrm{~h}, J=7.0 \mathrm{~Hz}, 1 \mathrm{H}), 2.95(\mathrm{dd}, J=16.6,3.9 \mathrm{~Hz}, 1 \mathrm{H}), 2.84-2.75(\mathrm{~m}$, $1 \mathrm{H}), 2.06-1.99(\mathrm{~m}, 1 \mathrm{H}), 1.82(\mathrm{qd}, J=12.9,5.2 \mathrm{~Hz}, 1 \mathrm{H}), 1.63(\mathrm{t}, J=12.6 \mathrm{~Hz}, 1 \mathrm{H}), 1.41$ 
(dd, $J=12.0,1.6 \mathrm{~Hz}, 1 \mathrm{H}), 1.32(\mathrm{~s}, 3 \mathrm{H}), 1.31(\mathrm{~s}, 3 \mathrm{H}), 1.26-1.23(\mathrm{~m}, 9 \mathrm{H}), 1.18(\mathrm{~s}, 3 \mathrm{H})$, $1.11(\mathrm{~s}, 3 \mathrm{H})$.

${ }^{13} \mathrm{C}$ NMR $\left(151 \mathrm{MHz}, \mathrm{CDCl}_{3}\right) \delta 143.5,142.3,129.9,129.4,121.9,120.4,118.7,116.1$, $99.8,99.4,77.4,64.7,58.5,51.0,48.2,47.9,38.7,38.3,37.2,30.8,28.9,27.1,22.1$, $22.1,21.4,17.9,17.9,15.3,15.0$.

HRMS (ES+) $\mathrm{m} / \mathrm{z}$ calc'd for $\mathrm{C}_{29} \mathrm{H}_{41} \mathrm{NO}_{7}[\mathrm{M}+\mathrm{Na}]^{+}: 538.2781$, found 538.2790.

TLC: $R_{f}=0.25$ (9:1 hexanes:EtOAc, visualized by CAM)

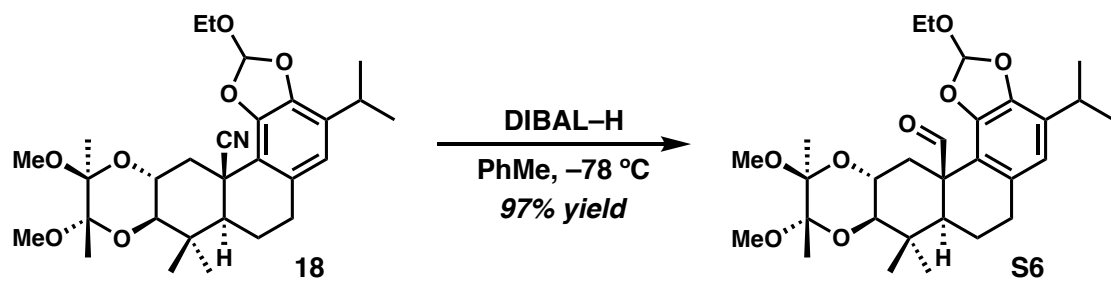

Nitrile 18 (29 mg, $0.058 \mathrm{mmol}, 1.0$ equiv.) was dissolved in dry $\mathrm{PhMe}(3.0 \mathrm{~mL})$ under $\mathrm{Ar}$ and cooled to $-78{ }^{\circ} \mathrm{C}$. DIBAL-H (0.5 M in PhMe, $0.93 \mathrm{~mL}, 0.47 \mathrm{mmol}, 8.0$ equiv.) was added dropwise by syringe. The resulting suspension was stirred at $-78{ }^{\circ} \mathrm{C}$ for 3 hours. The reaction mixture was warmed to $0^{\circ} \mathrm{C}$ and diluted with $\mathrm{PhMe}(3 \mathrm{~mL})$. The reaction was quenched with the addition of saturated aqueous Rochelle salt $(4 \mathrm{~mL})$. The biphasic solution was allowed to stir vigorously for 12 hours at room temperature. The organic layer was separated, acidified with $\mathrm{HCO}_{2} \mathrm{H}(1.0 \mathrm{~mL})$, and diluted with deionized water (1.0 $\mathrm{mL}$ ). The resulting biphasic solution was allowed to stir vigorously for 30 minutes. The organic layer was collected and washed with brine $(10 \mathrm{~mL})$, dried over sodium sulfate, and concentrated under reduced pressure to afford aldehyde $\mathbf{S 6}(28 \mathrm{mg}, 0.054 \mathrm{mmol}$, $97 \%$ ) as a white foam, which was used without further purification.

NMR data for this compound are reported for the compound derived from the less polar diastereomer $\mathbf{1 8 b}$ (at the orthoester).

${ }^{1} \mathrm{H}$ NMR $\left(500 \mathrm{MHz}, \mathrm{CDCl}_{3}\right) \delta 9.80(\mathrm{~s}, 1 \mathrm{H}), 6.81(\mathrm{~s}, 1 \mathrm{H}), 6.57(\mathrm{~s}, 1 \mathrm{H}), 3.93(\mathrm{td}, J=11.5$, $4.3 \mathrm{~Hz}, 1 \mathrm{H}), 3.59(\mathrm{q}, J=7.1 \mathrm{~Hz}, 2 \mathrm{H}), 3.51(\mathrm{dd}, J=12.7,4.4 \mathrm{~Hz}, 1 \mathrm{H}), 3.35(\mathrm{~s}, 3 \mathrm{H}), 3.27-$ $3.21(\mathrm{~m}, 4 \mathrm{H}), 3.06-2.95(\mathrm{~m}, 2 \mathrm{H}), 2.95-2.85(\mathrm{~m}, 1 \mathrm{H}), 2.12(\mathrm{qd}, J=12.9,5.3 \mathrm{~Hz}, 1 \mathrm{H})$, 1.97 (dd, $J=13.7,6.2 \mathrm{~Hz}, 1 \mathrm{H}), 1.68(\mathrm{~d}, J=12.5 \mathrm{~Hz}, 1 \mathrm{H}), 1.39(\mathrm{~d}, J=10.3 \mathrm{~Hz}, 2 \mathrm{H}), 1.31$ $(\mathrm{s}, 3 \mathrm{H}), 1.29(\mathrm{~s}, 3 \mathrm{H}), 1.27-1.19(\mathrm{~m}, 10 \mathrm{H}), 1.10(\mathrm{~s}, 3 \mathrm{H}), 0.86(\mathrm{~s}, 3 \mathrm{H})$.

${ }^{13} \mathrm{C}$ NMR $\left(151 \mathrm{MHz}, \mathrm{CDCl}_{3}\right) \delta 196.7,144.8,142.3,131.3,129.5,120.4,118.3,113.5$, $99.8,99.3,77.8,64.3,58.3,52.5,52.2,48.1,47.8,38.2,33.0,31.1,28.8,26.4,22.2,22.1$, 18.1 (2 C), 17.9, 16.3, 15.0.

HRMS (ES+) $m / z$ calc'd for $\mathrm{C}_{29} \mathrm{H}_{42} \mathrm{O}_{8}[\mathrm{M}+\mathrm{Na}]^{+}: 541.2777$, found 541.2792 .

TLC: $R_{f}=0.23$ (9:1 hexanes:EtOAc, visualized by CAM) 


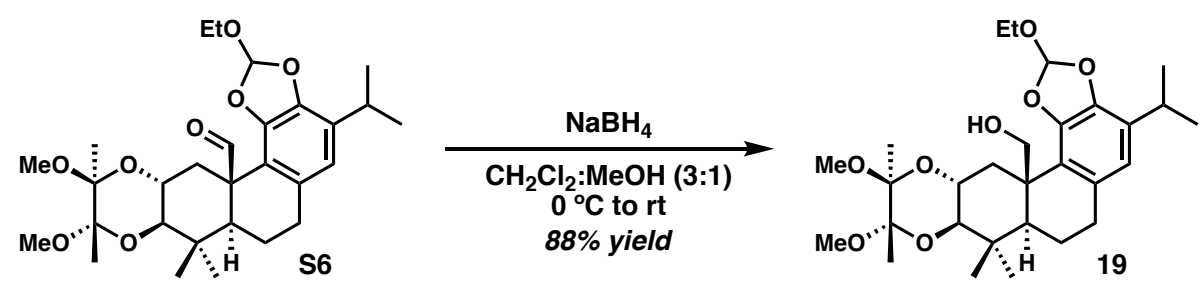

Aldehyde $\mathbf{S 6}$ (90 mg, $0.17 \mathrm{mmol}, 1.0$ equiv.) was dissolved in $\mathrm{CH}_{2} \mathrm{Cl}_{2}: \mathrm{MeOH}(3: 1,4.3 \mathrm{~mL}$ ) and cooled to $0^{\circ} \mathrm{C}$ in an ice bath. Sodium borohydride (33 mg, $0.87 \mathrm{mmol}, 5.0$ equiv.) was added in one portion and the resulting mixture allowed to stir for 3 hours. The reaction was quenched with the addition of EtOAc $(3 \mathrm{~mL})$ and saturated aqueous ammonium chloride $(5 \mathrm{~mL})$. The biphasic solution was stirred vigorously for 12 hours and then diluted with EtOAc $(12 \mathrm{~mL})$ and deionized water $(15 \mathrm{~mL})$. The organic layer was separated and washed with brine $(20 \mathrm{~mL})$, dried over sodium sulfate, and concentrated under reduced pressure. The crude residue was purified by flash column chromatography $\left(\mathrm{SiO}_{2}, 4: 1\right.$ hexanes:EtOAc), providing carbinol 19 (79 $\mathrm{mg}, 0.15 \mathrm{mmol}, 88 \%)$ as a translucent foam.

NMR data for this compound are reported for the compound derived from the less polar diastereomer $\mathbf{1 8 b}$ (at the orthoester).

${ }^{1} \mathrm{H}$ NMR $\left(600 \mathrm{MHz}, \mathrm{CDCl}_{3}\right) \delta 6.83(\mathrm{~s}, 1 \mathrm{H}), 6.53(\mathrm{~s}, 1 \mathrm{H}), 6.57(\mathrm{~s}, 1 \mathrm{H}), 4.00(\mathrm{~d}, J=11.4 \mathrm{~Hz}$, $1 \mathrm{H}$ ), $3.93(\mathrm{~d}, J=11.4 \mathrm{~Hz}, 1 \mathrm{H}), 3.93-3.87(\mathrm{~m}, 1 \mathrm{H}), 3.61$ (tdd, $J=19.6,9.0,6.6 \mathrm{~Hz}, 2 \mathrm{H}$ ), $3.30(\mathrm{~s}, 3 \mathrm{H}), 3.27-3.21(\mathrm{~m}, 4 \mathrm{H}), 3.11(\mathrm{dd}, J=13.1,4.1 \mathrm{~Hz}, 1 \mathrm{H}), 3.00(\mathrm{~h}, J=7.0 \mathrm{~Hz}, 1 \mathrm{H})$, $2.94(\mathrm{dd}, J=16.9,6.2 \mathrm{~Hz}, 1 \mathrm{H}), 2.87-2.79(\mathrm{~m}, 1 \mathrm{H}), 1.94(\mathrm{qd}, J=12.6,6.6 \mathrm{~Hz}, 1 \mathrm{H}), 1.8$ $(\mathrm{dd}, J=13.3,7.7 \mathrm{~Hz}, 1 \mathrm{H}), 1.57-1.50(\mathrm{~m}, 2 \mathrm{H}), 1.31(\mathrm{~s}, 3 \mathrm{H}), 1.30(\mathrm{~s}, 3 \mathrm{H}), 1.27-1.20(\mathrm{~m}$, $9 \mathrm{H}), 1.09(\mathrm{~s}, 3 \mathrm{H}), 1.01(\mathrm{~s}, 3 \mathrm{H})$.

${ }^{13} \mathrm{C}$ NMR $\left(151 \mathrm{MHz}, \mathrm{CDCl}_{3}\right) \delta 143.3,141.4,130.5,128.2,122.4,120.4,117.7,99.7,99.3$, $78.6,66.9,64.4,58.2,51.2,48.1,47.8,44.4,38.1,35.9,30.5,28.8,28.5,22.3,22.1,18.4$, $18.1,18.0,17.6,15.1$.

HRMS (ES+) $m / z$ calc'd for $\mathrm{C}_{29} \mathrm{H}_{44} \mathrm{O}_{8}[\mathrm{M}+\mathrm{Na}]^{+}: 543.2934$, found 543.2932.

TLC: $\mathrm{R}_{f}=0.21\left(96: 4 \mathrm{CH}_{2} \mathrm{Cl}_{2}:\right.$ EtOAc, visualized by CAM)

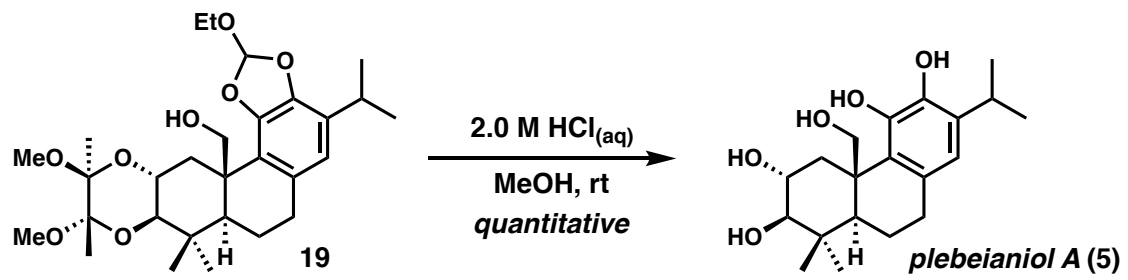

Carbinol 19 (9.2 mg, $18 \mu \mathrm{mol}, 1.0$ equiv.) was dissolved in $\mathrm{MeOH}(1.5 \mathrm{~mL}) . \mathrm{HCl}(2.0 \mathrm{M}$ aqueous, $1.5 \mathrm{~mL}$ ) was added. The reaction mixture was stirred at room temperature for 3 hours, producing a clear solution that was slowly concentrated under reduced pressure to afford plebeianiol A (5) $(6.2 \mathrm{mg}, 18 \mu \mathrm{mol},>99 \%)$ as a white film.

${ }^{1} \mathrm{H}$ NMR (500 MHz, DMSO-d d $\delta 9.63$ (br s, 1H), 7.54 (br s, 1H), 6.57 (br s, 1H), 6.36 (s, $1 \mathrm{H}), 4.11(\mathrm{~d}, J=10.0 \mathrm{~Hz}, 1 \mathrm{H}), 3.71(\mathrm{~d}, J=10.0 \mathrm{~Hz}, 1 \mathrm{H}), 3.58(\mathrm{ddd}, J=11.7,9.4,3.9 \mathrm{~Hz}$, 
1H), 3.34 (dd, $J=13.4,4.0 \mathrm{~Hz}, 1 \mathrm{H}), 3.11(\mathrm{~h}, J=7.0 \mathrm{~Hz}, 1 \mathrm{H}), 2.85(\mathrm{~d}, J=9.4 \mathrm{~Hz}, 1 \mathrm{H})$, $2.78-2.68(\mathrm{~m}, 2 \mathrm{H}), 1.71-1.59(\mathrm{~m}, 1 \mathrm{H}), 1.51(\mathrm{qd}, J=12.1,7.7 \mathrm{~Hz}, 1 \mathrm{H}), 1.28(\mathrm{~d}, J=12.0$ $\mathrm{Hz}, 1 \mathrm{H}), 1.12(\mathrm{~d}, J=6.8 \mathrm{~Hz}, 3 \mathrm{H}), 1.11(\mathrm{~d}, J=6.8 \mathrm{~Hz}, 3 \mathrm{H}), 1.01(\mathrm{~s}, 3 \mathrm{H}), 0.99-0.93(\mathrm{~m}, 1$ $\mathrm{H}), 0.78(\mathrm{~s}, 3 \mathrm{H})$.

${ }^{13} \mathrm{C}$ NMR $\left(151 \mathrm{MHz}\right.$, DMSO- $\left.d_{6}\right) \delta 143.0,141.7,132.1,130.2,126.3,117.4,81.9,67.0$, 65.4, 52.3, 44.3, 38.9, 38.1, 31.8, 29.7, 26.4, 22.5, 22.4, 18.2 (2 C).

HRMS (ES+) $\mathrm{m} / \mathrm{z}$ calc'd for $\mathrm{C}_{20} \mathrm{H}_{30} \mathrm{O}_{5}[\mathrm{M}+\mathrm{Na}]^{+}: 373.1991$, found 373.1991

TLC: $\mathrm{R}_{f}=0.20\left(92: 8 \mathrm{CH}_{2} \mathrm{Cl}_{2}: \mathrm{MeOH}\right.$, visualized by CAM)

$[\alpha]^{22.8} \mathrm{D}+26.2(\mathrm{c}=1.0, \mathrm{MeOH})$ [observed]

$[\alpha]^{27} \mathrm{D}+25.8(\mathrm{c}=0.1, \mathrm{MeOH})[$ literature 6$]$

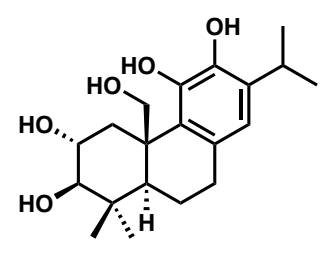

plebeianiol A (5)

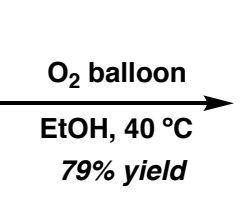

$2 a, 3 \beta, 11,12-t e t r a h y d r o x y-7 \beta$, 20-epoxy-8,11,13-abietatriene (6)

Plebeianiol A (5) (1.6 mg, $0.0046 \mathrm{mmol}, 1.0$ equiv.) was dissolved in $\mathrm{EtOH}(1.0 \mathrm{~mL})$. The vial was capped with a rubber septum. The septum was vented with a syringe needle and the solution was sparged under positive pressure with an $\mathrm{O}_{2}$ balloon for 5 minutes. The cap was replaced and the vial was sealed with electrical tape. The solution was placed in a pre-heated oil bath and stirred at $40{ }^{\circ} \mathrm{C}$ for 4 hours. The reaction mixture was concentrated under reduced pressure and purified by flash column chromatography $\left(\mathrm{SiO}_{2}, 1: 9 \mathrm{EtOH}: \mathrm{PhH}\right.$ with $\left.2 \% \mathrm{AcOH}\right)$ to give $6(1.1 \mathrm{mg}, 0.003 \mathrm{mmol}, 79 \%)$ as an orange oil.

The spectral data for 6 were in good accord with those previously published. ${ }^{7}$

${ }^{1} \mathrm{H}$ NMR $\left(600 \mathrm{MHz}, \mathrm{CDCl}_{3}\right) \delta 6.52(\mathrm{~s}, 1 \mathrm{H}), 4.61(\mathrm{~d}, J=3.5 \mathrm{~Hz}, 1 \mathrm{H}), 4.06(\mathrm{~d}, J=8.5 \mathrm{~Hz}$, $1 \mathrm{H}$ ), 3.49 (ddd, $J=11.9,9.3,4.2 \mathrm{~Hz}, 1 \mathrm{H}$ ), $3.23(\mathrm{~h}, J=6.8 \mathrm{~Hz}, 1 \mathrm{H}), 2.88$ (dd, $J=9.1,3.4$ $\mathrm{Hz}, 2 \mathrm{H}$ ), 2.68 (dd, $J=14.3,11.9 \mathrm{~Hz}, 1 \mathrm{H}$ ), 2.11 (dd, $J=14.2,4.3 \mathrm{~Hz}, 1 \mathrm{H}), 1.92$ (dd, $J=$ 7.6, 3.8 Hz, $1 \mathrm{H}), 1.43-1.36(\mathrm{~m}, 2 \mathrm{H}), 1.13(\mathrm{~d}, J=6.8 \mathrm{~Hz}, 3 \mathrm{H}), 1.11(\mathrm{~d}, J=6.8 \mathrm{~Hz}, 3 \mathrm{H})$, $0.98(\mathrm{~s}, 3 \mathrm{H}), 0.86(\mathrm{~s}, 3 \mathrm{H})$.

${ }^{13} \mathrm{C}$ NMR $\left(151 \mathrm{MHz}, \mathrm{CDCl}_{3}\right) \delta 142.3,140.7,133.2,132.0,128.1,111.2,81.6,69.6,68.6$, 67.0, 43.0, 39.9, 39.3, 37.2, 29.4, 28.9, 26.2, 22.9, 22.8, 15.8.

HRMS (ES-) $m / z$ calc'd for $\mathrm{C}_{20} \mathrm{H}_{28} \mathrm{O}_{5}[\mathrm{M}-\mathrm{H}]^{-}: 347.1859$, found 347.1866.

TLC: $\mathrm{R}_{f}=0.20$ (1:9 EtOH:PhH with $2 \% \mathrm{AcOH}$, visualized by CAM)

$[\alpha]^{22} \mathrm{D}-19.4(\mathrm{c}=0.5, i-\mathrm{PrOH})$

Note: There is no optical rotation data for this compound reported in the literature. 


\section{Method for Predicting ${ }^{13} \mathrm{C}$ Shifts}

All calculations were performed using Spartan $18^{8}$ on structures $\mathbf{4}$ and $\mathbf{5}$ in the gas phase. First, a distribution of conformers was calculated with Molecular Mechanics MMFF. All conformers exceeding $13 \mathrm{kcal} / \mathrm{mol}$ were discarded. The remaining conformers were subjected to an equilibrium geometry calculation using HF/3-21G and then assigned an alignment score. Any duplicates (conformers that shared an align score and relative energy) were discarded as well as any conformers exceeding $5 \mathrm{kcal} / \mathrm{mol}$. All remaining conformers were refined using a single point energy calculation with $\omega B$ 97X-D/6-31G* and all conformers exceeding $3 \mathrm{kcal} / \mathrm{mol}$ were discarded. [For example for 5]: At this point 9 conformers remained, and they were subjected to a final energy refinement with $\omega$ B97X$\mathrm{D} / 6-311+\mathrm{G}(2 \mathrm{df}, 2 \mathrm{p})\left[6-311 \mathrm{G}^{*}\right]$ with NMR prediction using $\omega \mathrm{B} 97 \mathrm{X}-\mathrm{D} / 6-31 \mathrm{G}^{*}$. Each of the 9 conformers were assigned a Boltzmann weight and the Boltzmann averaged ${ }^{13} \mathrm{C}$ shifts are reported for $\mathbf{5}$ in Table $\mathbf{S 2}$.

\section{References}

1. Glover, S.; Hammond, G.; Harman, D.; Mills, J.; Rowbottom, C. Aust. J. Chem. 1993, 46, 1213-1228.

2. Kolb, H. C.; VanNieuwenhze, M. S.; Sharpless, K. B. Chem. Rev. 1994, 94, 2483-2547.

3. Stowers, K. J.; Fortner, K. C.; Sanford, M. S. J. Am. Chem. Soc. 2011, 133, 6541-6544.

4. Nikas, S. P.; Thakur, G. P.; Makriyannis, A. Synth-Commun. 2002, 32, 1751-1756.

5. Vrubliauskas, D.; Gross, B. M.; Vanderwal, C. D. J. Am. Chem. Soc. 2021, 143, 2944-2952.

6. Zhang, B.-B.; He, B.-Q.; Sun, J.-B.; Zeng, B.; Shi, X.-J.; Zhou, Y.; Niu, Y.; Nie, S.-Q.; Feng, F.; Liang, Y.; Wu, F.-H. Molecules 2015, 20, 14879-14888.

7. Xiong, J.; Qu, W.; Sun, J.; Wang, M.; Liang, J. Phytochemistry Letters 2013, 6, 457-460.

8. (a) Spartan 18, Wavefunction Inc., Irvine CA. (b) Shao, Y.; Gan, Z.; Epifanovsky, E.; Gilbert, A. T. B.; Wormit, M.; Kussmann, J.; Lange, A. W.; Behn, A.; Deng, J.; Feng, X.; Ghosh, D.; Goldey, M.; Horn, P. R.; Jacobson, L. D.; Kaliman, I.; Khaliullin, R. Z.; Kuś, T.; Landau, A.; Liu, J.; Proynov, E. I.; Rhee, Y. M.; Richard, R. M.; Rohrdanz, M. A.; Steele, R. P.; Sundstrom, E. J.; Woodcock, H. L., Ill; Zimmerman, P. M.; Zuev, D.; Albrecht, B.; Alguire, E.; Austin, B.; Beran, G. J. O.; Bernard, Y. A.; Berquist, E.; Brandhorst, K.; Bravaya, K. B.; Brown, S. T.; Casanova, D.; Chang, C.-M.; Chen, Y.; Chien, S. H.; Closser, K. D.; Crittenden, D. L.; Diedenhofen, M.; DiStasio, R. A., Jr.; Do, H.; Dutoi, A. D.; Edgar, R. G.; Fatehi, S.; FustiMolnar, L.; Ghysels, A.; Golubeva-Zadorozhnaya, A.; Gomes, J.; Hanson-Heine, M. W. D.; Harbach, P. H. P.; Hauser, A. W.; Hohenstein, E. G.; Holden, Z. C.; Jagau, T.-C.; Ji, H.; Kaduk, B.; Khistyaev, K.; Kim, J.; Kim, J.; King, R. A.; Klunzinger, P.; Kosenkov, D.; Kowalczyk, T.; Krauter, C. M.; Lao, K. U.; Laurent, A. D.; Lawler, K. V.; Levchenko, S. V.; Lin, C. Y.; Liu, F.; Livshits, E.; Lochan, R. C.; Luenser, A.; Manohar, P.; Manzer, S. F.; Mao, S.-P.; Mardirossian, N.; Marenich, A. V.; Maurer, S. A.; Mayhall, N. J.; Neuscamman, E.; Oana, C. M.; OlivaresAmaya, R.; O'Neill, D. P.; Parkhill, J. A.; Perrine, T. M.; Peverati, R.; Prociuk, A.; Rehn, D. R.; Rosta, E.; Russ, N. J.; Sharada, S. M.; Sharma, S.; Small, D. W.; Sodt, A.; Stein, T.; Stück, D.; Su, Y.-C.; Thom, A. J. W.; Tsuchimochi, T.; Vanovschi, V.; Vogt, L.; Vydrov, O.; Wang, T.; Watson, M. A.; Wenzel, J.; White, A.; Williams, C. F.; Yang, J.; Yeganeh, S.; Yost, S. R.; You, Z.-Q.; Zhang, I. Y.; Zhang, X.; Zhao, Y.; Brooks, B. R.; Chan, G. K. L.; Chipman, D. M.; Cramer, C. J.; Goddard lii, W. A.; Gordon, M. S.; Hehre, W. J.; Klamt, A.; Schaefer, H. F., III; 
Schmidt, M. W.; Sherrill, C. D.; Truhlar, D. G.; Warshel, A.; Xu, X.; Aspuru-Guzik, A.; Baer, R.; Bell, A. T.; Besley, N. A.; Chai, J.-D.; Dreuw, A.; Dunietz, B. D.; Furlani, T. R.; Gwaltney, S. R.; Hsu, C.-P.; Jung, Y.; Kong, J.; Lambrecht, D. S.; Liang, W.; Ochsenfeld, C.; Rassolov, V. A.; Slipchenko, L. V.; Subotnik, J. E.; Van Voorhis, T.; Herbert, J. M.; Krylov, A. I.; Gill, P. M. W.; Head-Gordon, M. Advances in Molecular Quantum Chemistry Contained in the QChem 4 Program Package. Molecular Physics 2014, 113, 184-215. 


\section{Experimental Data}

\section{$\underline{X-r a y ~ D a t a ~ f o r ~ S u b s t r a t e s ~ 22 a-c}$}

X-ray Data Collection, Structure Solution and Refinement for BDA protected 22a (cdv87).

A colorless crystal of approximate dimensions $0.067 \times 0.089 \times 0.237 \mathrm{~mm}$ was mounted in a cryoloop and transferred to a Bruker Prospector APEX II diffractometer system. The APEX $3^{1}$ program package was used to determine the unit-cell parameters and for data collection (3-6 sec/frame scan time). The raw frame data was processed using SAINT2 and $\mathrm{SADABS}^{3}$ to yield the reflection data file. Subsequent calculations were carried out using the SHELXTL ${ }^{4}$ program package. The diffraction symmetry was $\mathrm{mmm}$ and the systematic absences were consistent with the orthorhombic space group $P 2{ }_{1} 2{ }_{1}{ }_{1}$ that was later determined to be correct.

The structure was solved by direct methods and refined on $\mathrm{F}^{2}$ by full-matrix least-squares techniques. The analytical scattering factors 5 for neutral atoms were used throughout the analysis. Hydrogen atoms were included using a riding model. There were two molecules of the formula-unit present.

Least squares analysis yielded $w R 2=0.0733$ and Goof $=1.065$ for 593 variables refined against 8832 data $(0.83 \AA)$, R1 $=0.0297$ for those 8294 data with $I>2.0 \sigma(I)$. The absolute structure was assigned by refinement of the Flack parameter ${ }^{6}$ and was in agreement with the synthetic method employed.

References.

1. APEX2 Version 2017.3-0, Bruker AXS, Inc.; Madison, WI 2017.

2. SAINT Version 8.38a, Bruker AXS, Inc.; Madison, WI 2013.

3. Sheldrick, G. M. SADABS, Version 2014/5, Bruker AXS, Inc.; Madison, WI 2014.

4. Sheldrick, G. M. SHELXTL, Version 2014/7, Bruker AXS, Inc.; Madison, WI 2014.

5. International Tables for Crystallography 1992, Vol. C., Dordrecht: Kluwer Academic Publishers.

6. Parsons, S., Flack, H. D., Wagner, T. Acta Cryst. B 2013, 69, 249-259. 


\section{Definitions:}

$\mathrm{wR} 2=\left[\Sigma\left[\mathrm{w}\left(\mathrm{F}_{\mathrm{o}}^{2}-\mathrm{F}_{\mathrm{c}}^{2}\right)^{2}\right] / \Sigma\left[\mathrm{w}\left(\mathrm{F}_{\mathrm{o}}^{2}\right)^{2}\right]\right]^{1 / 2}$

$\mathrm{R} 1=\Sigma|| F_{\mathrm{o}}|-| \mathrm{F}_{\mathrm{c}} \| / \Sigma\left|\mathrm{F}_{\mathrm{o}}\right|$

Goof $=S=\left[\Sigma\left[w\left(F_{o}^{2}-F_{c}^{2}\right)^{2}\right] /(n-p)\right]^{1 / 2}$ where $n$ is the number of reflections and $p$ is the total number of parameters refined.

The thermal ellipsoid plot is shown at the $50 \%$ probability level. 

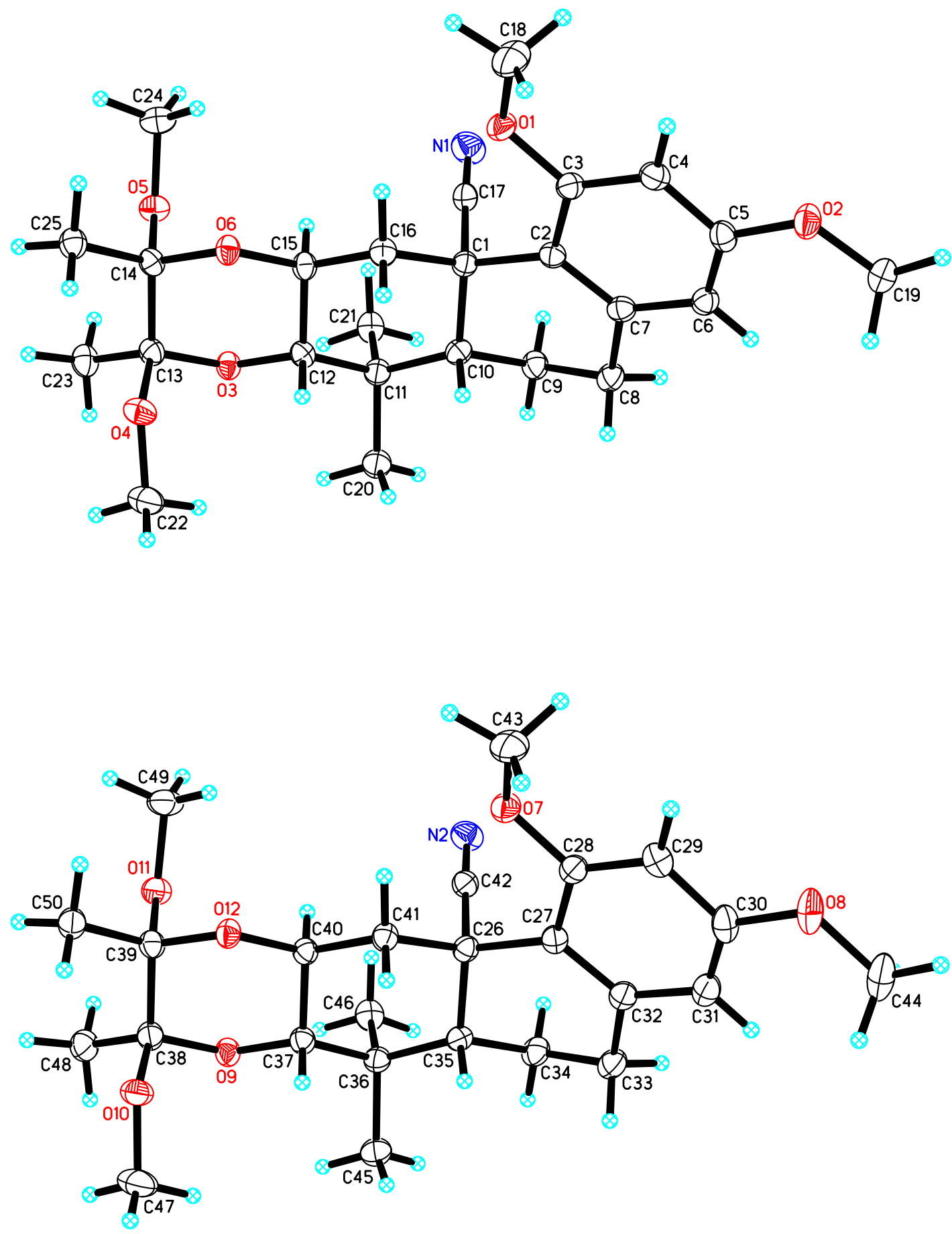
Table 1. Crystal data and structure refinement for $\operatorname{cdv} 87$.

Identification code

Empirical formula

Formula weight

Temperature

Wavelength

Crystal system

Space group

Unit cell dimensions

Volume

Z

Density (calculated)

Absorption coefficient

$\mathrm{F}(000)$

Crystal color

Crystal size

Theta range for data collection

Index ranges

Reflections collected

Independent reflections

Completeness to theta $=67.679^{\circ}$

Absorption correction

Max. and min. transmission

Refinement method

Data / restraints / parameters

Goodness-of-fit on $\mathrm{F}^{2}$

Final $\mathrm{R}$ indices $[\mathrm{I}>2 \operatorname{sigma}(\mathrm{I})=8294$ data $]$

$\mathrm{R}$ indices (all data, $0.83 \AA$ )

Absolute structure parameter

Largest diff. peak and hole $\operatorname{cdv} 87$ (Scott Niman)

$\mathrm{C}_{25} \mathrm{H}_{35} \mathrm{~N} \mathrm{O}_{6}$

445.54

92(2) K

$1.54178 \AA$

Orthorhombic

$P 2{ }_{1} 2_{1} 2_{1}$

$\mathrm{a}=7.0159(6) \AA \quad \alpha=90^{\circ}$.

$\mathrm{b}=16.4284(14) \AA \quad \beta=90^{\circ}$.

$\mathrm{c}=41.274(4) \AA \quad \gamma=90^{\circ}$.

4757.3(7) $\AA^{3}$

8

$1.244 \mathrm{Mg} / \mathrm{m}^{3}$

$0.718 \mathrm{~mm}^{-1}$

1920

colorless

$0.237 \times 0.089 \times 0.067 \mathrm{~mm}^{3}$

2.895 to $68.961^{\circ}$

$-8 \leq h \leq 8,-19 \leq k \leq 19,-47 \leq l \leq 49$

188983

$8832[\mathrm{R}(\mathrm{int})=0.0673]$

$100.0 \%$

Semi-empirical from equivalents

0.8643 and 0.8048

Full-matrix least-squares on $\mathrm{F}^{2}$

8832 / 0 / 593

1.065

$\mathrm{R} 1=0.0297, \mathrm{wR} 2=0.0719$

$\mathrm{R} 1=0.0326, \mathrm{wR} 2=0.0733$

$0.04(5)$

0.134 and -0.199 e. $\AA^{-3}$ 
Table 2. Atomic coordinates $\left(\mathrm{x} 10^{4}\right)$ and equivalent isotropic displacement parameters $\left(\AA^{2} \times 10^{3}\right)$ for $\operatorname{cdv} 87 . \mathrm{U}(\mathrm{eq})$ is defined as one third of the trace of the orthogonalized $\mathrm{U}^{\mathrm{ij}}$ tensor.

\begin{tabular}{|c|c|c|c|c|}
\hline & $\mathrm{x}$ & $\mathrm{y}$ & $\mathrm{z}$ & $\mathrm{U}(\mathrm{eq})$ \\
\hline $\mathrm{N}(1)$ & 2857(3) & $3477(1)$ & $3413(1)$ & $31(1)$ \\
\hline $\mathrm{O}(1)$ & $6210(2)$ & $2816(1)$ & $4078(1)$ & $22(1)$ \\
\hline $\mathrm{O}(2)$ & $8170(2)$ & $5184(1)$ & $4633(1)$ & $23(1)$ \\
\hline $\mathrm{O}(3)$ & $7680(2)$ & $2247(1)$ & $2543(1)$ & $18(1)$ \\
\hline $\mathrm{O}(4)$ & $10274(2)$ & $1367(1)$ & $2609(1)$ & $22(1)$ \\
\hline $\mathrm{O}(5)$ & $5367(2)$ & $954(1)$ & $2758(1)$ & $20(1)$ \\
\hline $\mathrm{O}(6)$ & $7635(2)$ & 1401(1) & $3131(1)$ & $18(1)$ \\
\hline $\mathrm{C}(1)$ & $6618(3)$ & $3501(1)$ & $3445(1)$ & $17(1)$ \\
\hline$C(2)$ & 7061(3) & $3973(1)$ & $3762(1)$ & $17(1)$ \\
\hline$C(3)$ & $6845(3)$ & $3607(1)$ & $4072(1)$ & $19(1)$ \\
\hline$C(4)$ & $7218(3)$ & $4030(1)$ & $4353(1)$ & $20(1)$ \\
\hline$C(5)$ & 7798(3) & $4839(1)$ & $4339(1)$ & $20(1)$ \\
\hline$C(6)$ & 7951(3) & $5224(1)$ & $4043(1)$ & 19(1) \\
\hline$C(7)$ & 7582(3) & 4794(1) & $3757(1)$ & $19(1)$ \\
\hline $\mathrm{C}(8)$ & 7815(3) & $5260(1)$ & $3444(1)$ & $22(1)$ \\
\hline $\mathrm{C}(9)$ & $6870(3)$ & $4849(1)$ & $3156(1)$ & $22(1)$ \\
\hline $\mathrm{C}(10)$ & $7510(3)$ & $3961(1)$ & $3150(1)$ & $18(1)$ \\
\hline $\mathrm{C}(11)$ & $7322(3)$ & $3552(1)$ & $2810(1)$ & $20(1)$ \\
\hline$C(12)$ & 7979(3) & $2666(1)$ & $2846(1)$ & $17(1)$ \\
\hline$C(13)$ & $8284(3)$ & $1422(1)$ & $2550(1)$ & $18(1)$ \\
\hline$C(14)$ & $7335(3)$ & $965(1)$ & $2838(1)$ & $17(1)$ \\
\hline$C(15)$ & 6972(3) & $2226(1)$ & $3115(1)$ & $17(1)$ \\
\hline$C(16)$ & 7388(3) & $2617(1)$ & $3438(1)$ & $19(1)$ \\
\hline$C(17)$ & 4497(3) & $3476(1)$ & $3423(1)$ & $20(1)$ \\
\hline$C(18)$ & $6277(4)$ & $2394(1)$ & $4380(1)$ & $33(1)$ \\
\hline$C(19)$ & $8603(3)$ & $6034(1)$ & $4638(1)$ & $23(1)$ \\
\hline$C(20)$ & $8735(3)$ & $3976(1)$ & $2580(1)$ & $25(1)$ \\
\hline$C(21)$ & $5308(3)$ & $3613(1)$ & $2663(1)$ & $23(1)$ \\
\hline$C(22)$ & $11475(3)$ & 1812(1) & 2392(1) & $28(1)$ \\
\hline$C(23)$ & $7740(3)$ & $1067(1)$ & $2223(1)$ & $24(1)$ \\
\hline$C(24)$ & $4147(3)$ & $585(1)$ & 2994(1) & $26(1)$ \\
\hline
\end{tabular}




\begin{tabular}{|c|c|c|c|c|}
\hline$C(25)$ & $8137(3)$ & $123(1)$ & $2898(1)$ & $20(1)$ \\
\hline $\mathrm{N}(2)$ & $7578(2)$ & 7643(1) & $4139(1)$ & $27(1)$ \\
\hline $\mathrm{O}(7)$ & $4271(2)$ & $6843(1)$ & $4777(1)$ & $22(1)$ \\
\hline $\mathrm{O}(8)$ & $2505(2)$ & $4137(1)$ & $4519(1)$ & $30(1)$ \\
\hline $\mathrm{O}(9)$ & $2455(2)$ & $9874(1)$ & $3820(1)$ & $20(1)$ \\
\hline $\mathrm{O}(10)$ & $-232(2)$ & $10338(1)$ & $4102(1)$ & $24(1)$ \\
\hline $\mathrm{O}(11)$ & $4612(2)$ & $10478(1)$ & $4349(1)$ & $22(1)$ \\
\hline $\mathrm{O}(12)$ & $2405(2)$ & $9466(1)$ & $4478(1)$ & $18(1)$ \\
\hline$C(26)$ & $3849(3)$ & $7442(1)$ & $4134(1)$ & $18(1)$ \\
\hline $\mathrm{C}(27)$ & $3526(3)$ & $6539(1)$ & $4230(1)$ & $18(1)$ \\
\hline$C(28)$ & $3710(3)$ & $6270(1)$ & $4554(1)$ & $19(1)$ \\
\hline$C(29)$ & $3378(3)$ & $5472(1)$ & $4642(1)$ & $22(1)$ \\
\hline$C(30)$ & 2859(3) & 4906(1) & $4406(1)$ & $23(1)$ \\
\hline$C(31)$ & 2757(3) & $5137(1)$ & $4086(1)$ & $23(1)$ \\
\hline$C(32)$ & $3108(3)$ & $5944(1)$ & 3998(1) & $20(1)$ \\
\hline$C(33)$ & 2984(3) & $6140(1)$ & $3640(1)$ & $24(1)$ \\
\hline$C(34)$ & $3833(3)$ & 6962(1) & $3555(1)$ & $23(1)$ \\
\hline$C(35)$ & $3006(3)$ & 7593(1) & $3788(1)$ & $18(1)$ \\
\hline$C(36)$ & $3069(3)$ & $8482(1)$ & $3657(1)$ & $20(1)$ \\
\hline$C(37)$ & 2251(3) & $9040(1)$ & $3922(1)$ & $19(1)$ \\
\hline $\mathrm{C}(38)$ & 1741(3) & $10447(1)$ & 4049(1) & $21(1)$ \\
\hline C(39) & $2646(3)$ & $10296(1)$ & $4389(1)$ & $19(1)$ \\
\hline$C(40)$ & $3205(3)$ & $8917(1)$ & $4243(1)$ & $17(1)$ \\
\hline$C(41)$ & 2891(3) & $8060(1)$ & $4365(1)$ & $19(1)$ \\
\hline$C(42)$ & $5950(3)$ & $7568(1)$ & $4136(1)$ & $20(1)$ \\
\hline$C(43)$ & 3912(3) & $6670(1)$ & $5111(1)$ & $30(1)$ \\
\hline$C(44)$ & 1946(3) & $3541(1)$ & $4283(1)$ & $33(1)$ \\
\hline$C(45)$ & $1727(3)$ & $8539(1)$ & $3363(1)$ & $28(1)$ \\
\hline$C(46)$ & $5072(3)$ & $8752(1)$ & $3552(1)$ & $25(1)$ \\
\hline$C(47)$ & $-1424(3)$ & $10396(2)$ & $3823(1)$ & $33(1)$ \\
\hline$C(48)$ & $2225(3)$ & $11276(1)$ & $3907(1)$ & $26(1)$ \\
\hline$C(49)$ & $5776(3)$ & 10322(1) & $4628(1)$ & $28(1)$ \\
\hline$C(50)$ & $1730(3)$ & $10793(1)$ & $4657(1)$ & $23(1)$ \\
\hline
\end{tabular}


Table 3. Bond lengths $[\AA]$ and angles $\left[{ }^{\circ}\right]$ for $\operatorname{cdv} 87$.

\begin{tabular}{|c|c|}
\hline $\mathrm{N}(1)-\mathrm{C}(17)$ & $1.151(3)$ \\
\hline $\mathrm{O}(1)-\mathrm{C}(3)$ & $1.374(2)$ \\
\hline $\mathrm{O}(1)-\mathrm{C}(18)$ & $1.426(2)$ \\
\hline $\mathrm{O}(2)-\mathrm{C}(5)$ & $1.365(2)$ \\
\hline $\mathrm{O}(2)-\mathrm{C}(19)$ & $1.429(2)$ \\
\hline $\mathrm{O}(3)-\mathrm{C}(13)$ & $1.421(2)$ \\
\hline $\mathrm{O}(3)-\mathrm{C}(12)$ & $1.442(2)$ \\
\hline $\mathrm{O}(4)-\mathrm{C}(13)$ & $1.420(2)$ \\
\hline $\mathrm{O}(4)-\mathrm{C}(22)$ & $1.432(2)$ \\
\hline $\mathrm{O}(5)-\mathrm{C}(14)$ & $1.420(2)$ \\
\hline $\mathrm{O}(5)-\mathrm{C}(24)$ & $1.432(2)$ \\
\hline $\mathrm{O}(6)-\mathrm{C}(14)$ & $1.423(2)$ \\
\hline $\mathrm{O}(6)-\mathrm{C}(15)$ & $1.434(2)$ \\
\hline $\mathrm{C}(1)-\mathrm{C}(17)$ & $1.492(3)$ \\
\hline$C(1)-C(16)$ & $1.550(3)$ \\
\hline $\mathrm{C}(1)-\mathrm{C}(2)$ & $1.553(3)$ \\
\hline$C(1)-C(10)$ & $1.563(3)$ \\
\hline$C(2)-C(7)$ & $1.398(3)$ \\
\hline $\mathrm{C}(2)-\mathrm{C}(3)$ & $1.420(3)$ \\
\hline$C(3)-C(4)$ & $1.378(3)$ \\
\hline$C(4)-C(5)$ & $1.392(3)$ \\
\hline$C(5)-C(6)$ & $1.379(3)$ \\
\hline$C(6)-C(7)$ & $1.401(3)$ \\
\hline$C(7)-C(8)$ & $1.512(3)$ \\
\hline $\mathrm{C}(8)-\mathrm{C}(9)$ & $1.516(3)$ \\
\hline$C(9)-C(10)$ & $1.527(3)$ \\
\hline$C(10)-C(11)$ & $1.561(3)$ \\
\hline$C(11)-C(12)$ & $1.532(3)$ \\
\hline$C(11)-C(20)$ & $1.540(3)$ \\
\hline$C(11)-C(21)$ & $1.541(3)$ \\
\hline$C(12)-C(15)$ & $1.503(2)$ \\
\hline$C(13)-C(23)$ & $1.517(3)$ \\
\hline$C(13)-C(14)$ & $1.554(3)$ \\
\hline$C(14)-C(25)$ & $1.514(3)$ \\
\hline
\end{tabular}




\begin{tabular}{|c|c|}
\hline$C(15)-C(16)$ & $1.511(2)$ \\
\hline $\mathrm{N}(2)-\mathrm{C}(42)$ & $1.149(3)$ \\
\hline $\mathrm{O}(7)-\mathrm{C}(28)$ & $1.374(2)$ \\
\hline $\mathrm{O}(7)-\mathrm{C}(43)$ & $1.429(2)$ \\
\hline $\mathrm{O}(8)-\mathrm{C}(30)$ & $1.369(2)$ \\
\hline $\mathrm{O}(8)-\mathrm{C}(44)$ & $1.435(3)$ \\
\hline $\mathrm{O}(9)-\mathrm{C}(38)$ & $1.426(2)$ \\
\hline $\mathrm{O}(9)-\mathrm{C}(37)$ & $1.439(2)$ \\
\hline $\mathrm{O}(10)-\mathrm{C}(38)$ & $1.413(2)$ \\
\hline $\mathrm{O}(10)-\mathrm{C}(47)$ & $1.428(2)$ \\
\hline $\mathrm{O}(11)-\mathrm{C}(39)$ & $1.420(2)$ \\
\hline $\mathrm{O}(11)-\mathrm{C}(49)$ & $1.435(2)$ \\
\hline $\mathrm{O}(12)-\mathrm{C}(39)$ & $1.424(2)$ \\
\hline $\mathrm{O}(12)-\mathrm{C}(40)$ & $1.439(2)$ \\
\hline$C(26)-C(42)$ & $1.489(3)$ \\
\hline$C(26)-C(41)$ & $1.546(3)$ \\
\hline$C(26)-C(27)$ & $1.553(3)$ \\
\hline$C(26)-C(35)$ & $1.566(2)$ \\
\hline$C(27)-C(32)$ & $1.399(3)$ \\
\hline$C(27)-C(28)$ & $1.413(3)$ \\
\hline$C(28)-C(29)$ & $1.381(3)$ \\
\hline$C(29)-C(30)$ & $1.394(3)$ \\
\hline$C(30)-C(31)$ & $1.377(3)$ \\
\hline$C(31)-C(32)$ & $1.397(3)$ \\
\hline$C(32)-C(33)$ & $1.512(3)$ \\
\hline$C(33)-C(34)$ & $1.516(3)$ \\
\hline$C(34)-C(35)$ & $1.527(3)$ \\
\hline$C(35)-C(36)$ & $1.557(3)$ \\
\hline$C(36)-C(46)$ & $1.536(3)$ \\
\hline$C(36)-C(37)$ & $1.538(3)$ \\
\hline$C(36)-C(45)$ & $1.539(3)$ \\
\hline$C(37)-C(40)$ & $1.500(3)$ \\
\hline$C(38)-C(48)$ & $1.521(3)$ \\
\hline$C(38)-C(39)$ & $1.560(3)$ \\
\hline$C(39)-C(50)$ & $1.519(3)$ \\
\hline $\mathrm{C}(40)-\mathrm{C}(41)$ & $1.511(3)$ \\
\hline
\end{tabular}




\begin{tabular}{|c|c|}
\hline $\mathrm{C}(3)-\mathrm{O}(1)-\mathrm{C}(18)$ & $117.76(15)$ \\
\hline $\mathrm{C}(5)-\mathrm{O}(2)-\mathrm{C}(19)$ & $117.36(15)$ \\
\hline $\mathrm{C}(13)-\mathrm{O}(3)-\mathrm{C}(12)$ & $113.22(13)$ \\
\hline $\mathrm{C}(13)-\mathrm{O}(4)-\mathrm{C}(22)$ & $115.94(15)$ \\
\hline $\mathrm{C}(14)-\mathrm{O}(5)-\mathrm{C}(24)$ & $115.38(14)$ \\
\hline $\mathrm{C}(14)-\mathrm{O}(6)-\mathrm{C}(15)$ & $112.72(13)$ \\
\hline$C(17)-C(1)-C(16)$ & $108.75(16)$ \\
\hline $\mathrm{C}(17)-\mathrm{C}(1)-\mathrm{C}(2)$ & $105.36(15)$ \\
\hline$C(16)-C(1)-C(2)$ & $114.36(15)$ \\
\hline $\mathrm{C}(17)-\mathrm{C}(1)-\mathrm{C}(10)$ & $111.39(16)$ \\
\hline$C(16)-C(1)-C(10)$ & $107.44(15)$ \\
\hline $\mathrm{C}(2)-\mathrm{C}(1)-\mathrm{C}(10)$ & $109.59(15)$ \\
\hline$C(7)-C(2)-C(3)$ & $116.74(17)$ \\
\hline$C(7)-C(2)-C(1)$ & $121.42(16)$ \\
\hline $\mathrm{C}(3)-\mathrm{C}(2)-\mathrm{C}(1)$ & $121.73(16)$ \\
\hline $\mathrm{O}(1)-\mathrm{C}(3)-\mathrm{C}(4)$ & $121.50(17)$ \\
\hline $\mathrm{O}(1)-\mathrm{C}(3)-\mathrm{C}(2)$ & $116.91(16)$ \\
\hline $\mathrm{C}(4)-\mathrm{C}(3)-\mathrm{C}(2)$ & $121.57(17)$ \\
\hline$C(3)-C(4)-C(5)$ & $120.20(18)$ \\
\hline $\mathrm{O}(2)-\mathrm{C}(5)-\mathrm{C}(6)$ & $125.56(18)$ \\
\hline $\mathrm{O}(2)-\mathrm{C}(5)-\mathrm{C}(4)$ & $114.59(17)$ \\
\hline$C(6)-C(5)-C(4)$ & $119.85(17)$ \\
\hline$C(5)-C(6)-C(7)$ & $120.02(18)$ \\
\hline$C(2)-C(7)-C(6)$ & $121.53(17)$ \\
\hline$C(2)-C(7)-C(8)$ & $121.96(17)$ \\
\hline$C(6)-C(7)-C(8)$ & $116.50(17)$ \\
\hline $\mathrm{C}(7)-\mathrm{C}(8)-\mathrm{C}(9)$ & $113.36(16)$ \\
\hline C(8)-C(9)-C(10) & $108.08(16)$ \\
\hline$C(9)-C(10)-C(11)$ & $113.74(15)$ \\
\hline $\mathrm{C}(9)-\mathrm{C}(10)-\mathrm{C}(1)$ & $109.27(15)$ \\
\hline$C(11)-C(10)-C(1)$ & $117.24(15)$ \\
\hline$C(12)-C(11)-C(20)$ & $107.16(16)$ \\
\hline$C(12)-C(11)-C(21)$ & $112.05(16)$ \\
\hline$C(20)-C(11)-C(21)$ & $108.50(16)$ \\
\hline$C(12)-C(11)-C(10)$ & $107.34(14)$ \\
\hline
\end{tabular}




\begin{tabular}{|c|c|}
\hline$C(20)-C(11)-C(10)$ & $107.75(16)$ \\
\hline$C(21)-C(11)-C(10)$ & $113.76(16)$ \\
\hline $\mathrm{O}(3)-\mathrm{C}(12)-\mathrm{C}(15)$ & $110.00(15)$ \\
\hline $\mathrm{O}(3)-\mathrm{C}(12)-\mathrm{C}(11)$ & $109.07(14)$ \\
\hline$C(15)-C(12)-C(11)$ & $112.70(15)$ \\
\hline $\mathrm{O}(4)-\mathrm{C}(13)-\mathrm{O}(3)$ & $110.92(15)$ \\
\hline $\mathrm{O}(4)-\mathrm{C}(13)-\mathrm{C}(23)$ & $112.13(16)$ \\
\hline $\mathrm{O}(3)-\mathrm{C}(13)-\mathrm{C}(23)$ & $105.85(15)$ \\
\hline $\mathrm{O}(4)-\mathrm{C}(13)-\mathrm{C}(14)$ & $104.96(15)$ \\
\hline $\mathrm{O}(3)-\mathrm{C}(13)-\mathrm{C}(14)$ & $110.38(15)$ \\
\hline$C(23)-C(13)-C(14)$ & $112.71(16)$ \\
\hline $\mathrm{O}(5)-\mathrm{C}(14)-\mathrm{O}(6)$ & $110.40(15)$ \\
\hline $\mathrm{O}(5)-\mathrm{C}(14)-\mathrm{C}(25)$ & $112.81(16)$ \\
\hline $\mathrm{O}(6)-\mathrm{C}(14)-\mathrm{C}(25)$ & $105.30(14)$ \\
\hline $\mathrm{O}(5)-\mathrm{C}(14)-\mathrm{C}(13)$ & $104.18(14)$ \\
\hline $\mathrm{O}(6)-\mathrm{C}(14)-\mathrm{C}(13)$ & $110.18(15)$ \\
\hline $\mathrm{C}(25)-\mathrm{C}(14)-\mathrm{C}(13)$ & $114.06(16)$ \\
\hline $\mathrm{O}(6)-\mathrm{C}(15)-\mathrm{C}(12)$ & $109.75(15)$ \\
\hline $\mathrm{O}(6)-\mathrm{C}(15)-\mathrm{C}(16)$ & $107.27(14)$ \\
\hline$C(12)-C(15)-C(16)$ & $110.96(15)$ \\
\hline$C(15)-C(16)-C(1)$ & $110.29(15)$ \\
\hline $\mathrm{N}(1)-\mathrm{C}(17)-\mathrm{C}(1)$ & $177.8(2)$ \\
\hline $\mathrm{C}(28)-\mathrm{O}(7)-\mathrm{C}(43)$ & $117.37(15)$ \\
\hline $\mathrm{C}(30)-\mathrm{O}(8)-\mathrm{C}(44)$ & $116.62(17)$ \\
\hline $\mathrm{C}(38)-\mathrm{O}(9)-\mathrm{C}(37)$ & $113.53(14)$ \\
\hline $\mathrm{C}(38)-\mathrm{O}(10)-\mathrm{C}(47)$ & $116.02(15)$ \\
\hline $\mathrm{C}(39)-\mathrm{O}(11)-\mathrm{C}(49)$ & $115.02(15)$ \\
\hline $\mathrm{C}(39)-\mathrm{O}(12)-\mathrm{C}(40)$ & $112.24(14)$ \\
\hline $\mathrm{C}(42)-\mathrm{C}(26)-\mathrm{C}(41)$ & $109.53(16)$ \\
\hline $\mathrm{C}(42)-\mathrm{C}(26)-\mathrm{C}(27)$ & $105.99(15)$ \\
\hline $\mathrm{C}(41)-\mathrm{C}(26)-\mathrm{C}(27)$ & $113.99(15)$ \\
\hline $\mathrm{C}(42)-\mathrm{C}(26)-\mathrm{C}(35)$ & $110.99(15)$ \\
\hline $\mathrm{C}(41)-\mathrm{C}(26)-\mathrm{C}(35)$ & $107.09(15)$ \\
\hline $\mathrm{C}(27)-\mathrm{C}(26)-\mathrm{C}(35)$ & $109.30(15)$ \\
\hline $\mathrm{C}(32)-\mathrm{C}(27)-\mathrm{C}(28)$ & $116.71(17)$ \\
\hline$C(32)-C(27)-C(26)$ & $121.43(17)$ \\
\hline
\end{tabular}




\begin{tabular}{|c|c|}
\hline$C(28)-C(27)-C(26)$ & $121.82(16)$ \\
\hline $\mathrm{O}(7)-\mathrm{C}(28)-\mathrm{C}(29)$ & $121.51(17)$ \\
\hline $\mathrm{O}(7)-\mathrm{C}(28)-\mathrm{C}(27)$ & $116.48(16)$ \\
\hline $\mathrm{C}(29)-\mathrm{C}(28)-\mathrm{C}(27)$ & $122.00(18)$ \\
\hline $\mathrm{C}(28)-\mathrm{C}(29)-\mathrm{C}(30)$ & $119.56(19)$ \\
\hline $\mathrm{O}(8)-\mathrm{C}(30)-\mathrm{C}(31)$ & $124.86(19)$ \\
\hline $\mathrm{O}(8)-\mathrm{C}(30)-\mathrm{C}(29)$ & $115.12(18)$ \\
\hline $\mathrm{C}(31)-\mathrm{C}(30)-\mathrm{C}(29)$ & $120.02(19)$ \\
\hline $\mathrm{C}(30)-\mathrm{C}(31)-\mathrm{C}(32)$ & $120.15(19)$ \\
\hline $\mathrm{C}(31)-\mathrm{C}(32)-\mathrm{C}(27)$ & $121.38(18)$ \\
\hline $\mathrm{C}(31)-\mathrm{C}(32)-\mathrm{C}(33)$ & $116.48(17)$ \\
\hline $\mathrm{C}(27)-\mathrm{C}(32)-\mathrm{C}(33)$ & $122.13(17)$ \\
\hline $\mathrm{C}(32)-\mathrm{C}(33)-\mathrm{C}(34)$ & $113.13(16)$ \\
\hline $\mathrm{C}(33)-\mathrm{C}(34)-\mathrm{C}(35)$ & $108.06(16)$ \\
\hline $\mathrm{C}(34)-\mathrm{C}(35)-\mathrm{C}(36)$ & $114.13(15)$ \\
\hline $\mathrm{C}(34)-\mathrm{C}(35)-\mathrm{C}(26)$ & $108.73(15)$ \\
\hline $\mathrm{C}(36)-\mathrm{C}(35)-\mathrm{C}(26)$ & $117.02(15)$ \\
\hline $\mathrm{C}(46)-\mathrm{C}(36)-\mathrm{C}(37)$ & $111.64(16)$ \\
\hline$C(46)-C(36)-C(45)$ & $108.69(16)$ \\
\hline $\mathrm{C}(37)-\mathrm{C}(36)-\mathrm{C}(45)$ & $107.18(16)$ \\
\hline $\mathrm{C}(46)-\mathrm{C}(36)-\mathrm{C}(35)$ & $113.23(16)$ \\
\hline $\mathrm{C}(37)-\mathrm{C}(36)-\mathrm{C}(35)$ & $107.61(15)$ \\
\hline $\mathrm{C}(45)-\mathrm{C}(36)-\mathrm{C}(35)$ & $108.27(16)$ \\
\hline $\mathrm{O}(9)-\mathrm{C}(37)-\mathrm{C}(40)$ & $110.01(15)$ \\
\hline $\mathrm{O}(9)-\mathrm{C}(37)-\mathrm{C}(36)$ & $108.82(14)$ \\
\hline $\mathrm{C}(40)-\mathrm{C}(37)-\mathrm{C}(36)$ & $112.42(15)$ \\
\hline $\mathrm{O}(10)-\mathrm{C}(38)-\mathrm{O}(9)$ & $111.39(15)$ \\
\hline $\mathrm{O}(10)-\mathrm{C}(38)-\mathrm{C}(48)$ & $113.09(17)$ \\
\hline $\mathrm{O}(9)-\mathrm{C}(38)-\mathrm{C}(48)$ & $104.91(15)$ \\
\hline $\mathrm{O}(10)-\mathrm{C}(38)-\mathrm{C}(39)$ & $103.77(15)$ \\
\hline $\mathrm{O}(9)-\mathrm{C}(38)-\mathrm{C}(39)$ & $110.42(15)$ \\
\hline $\mathrm{C}(48)-\mathrm{C}(38)-\mathrm{C}(39)$ & $113.42(16)$ \\
\hline $\mathrm{O}(11)-\mathrm{C}(39)-\mathrm{O}(12)$ & $110.30(15)$ \\
\hline $\mathrm{O}(11)-\mathrm{C}(39)-\mathrm{C}(50)$ & $112.46(16)$ \\
\hline $\mathrm{O}(12)-\mathrm{C}(39)-\mathrm{C}(50)$ & $105.96(15)$ \\
\hline $\mathrm{O}(11)-\mathrm{C}(39)-\mathrm{C}(38)$ & $105.00(15)$ \\
\hline
\end{tabular}




$\begin{array}{ll}\mathrm{O}(12)-\mathrm{C}(39)-\mathrm{C}(38) & 109.69(15) \\ \mathrm{C}(50)-\mathrm{C}(39)-\mathrm{C}(38) & 113.48(16) \\ \mathrm{O}(12)-\mathrm{C}(40)-\mathrm{C}(37) & 109.76(15) \\ \mathrm{O}(12)-\mathrm{C}(40)-\mathrm{C}(41) & 107.63(14) \\ \mathrm{C}(37)-\mathrm{C}(40)-\mathrm{C}(41) & 110.73(15) \\ \mathrm{C}(40)-\mathrm{C}(41)-\mathrm{C}(26) & 110.15(15) \\ \mathrm{N}(2)-\mathrm{C}(42)-\mathrm{C}(26) & 178.1(2)\end{array}$


Table 4. Anisotropic displacement parameters $\left(\AA^{2} \times 10^{3}\right)$ for cdv87. The anisotropic displacement factor exponent takes the form: $-2 \pi^{2}\left[h^{2} a^{* 2} U^{11}+\ldots+2 h k a^{*} b^{*} U^{12}\right]$

\begin{tabular}{|c|c|c|c|c|c|c|}
\hline & $\mathrm{U}^{11}$ & $\mathrm{U}^{22}$ & $\mathrm{U}^{33}$ & $\mathrm{U}^{23}$ & $\mathrm{U}^{13}$ & $\mathrm{U}^{12}$ \\
\hline $\mathrm{N}(1)$ & $22(1)$ & $40(1)$ & $31(1)$ & $-4(1)$ & $0(1)$ & $-1(1)$ \\
\hline $\mathrm{O}(1)$ & $30(1)$ & $18(1)$ & $18(1)$ & $1(1)$ & $1(1)$ & $-6(1)$ \\
\hline $\mathrm{O}(2)$ & $31(1)$ & $20(1)$ & $19(1)$ & $-3(1)$ & $-3(1)$ & $-2(1)$ \\
\hline $\mathrm{O}(3)$ & $22(1)$ & $17(1)$ & $15(1)$ & $-1(1)$ & $-1(1)$ & $1(1)$ \\
\hline $\mathrm{O}(4)$ & $18(1)$ & $26(1)$ & $22(1)$ & $4(1)$ & $2(1)$ & $2(1)$ \\
\hline $\mathrm{O}(5)$ & $18(1)$ & $24(1)$ & $20(1)$ & $2(1)$ & $-2(1)$ & $-1(1)$ \\
\hline $\mathrm{O}(6)$ & $22(1)$ & $16(1)$ & $15(1)$ & $-1(1)$ & $-2(1)$ & $2(1)$ \\
\hline $\mathrm{C}(1)$ & $15(1)$ & $17(1)$ & $20(1)$ & $-1(1)$ & $0(1)$ & $0(1)$ \\
\hline$C(2)$ & $15(1)$ & $18(1)$ & $19(1)$ & $-1(1)$ & $1(1)$ & $0(1)$ \\
\hline $\mathrm{C}(3)$ & $18(1)$ & $18(1)$ & $21(1)$ & $0(1)$ & $2(1)$ & $-1(1)$ \\
\hline$C(4)$ & $20(1)$ & $23(1)$ & $18(1)$ & 1(1) & $2(1)$ & $-1(1)$ \\
\hline $\mathrm{C}(5)$ & $17(1)$ & $23(1)$ & $19(1)$ & $-4(1)$ & $-1(1)$ & $2(1)$ \\
\hline$C(6)$ & $17(1)$ & $18(1)$ & $23(1)$ & $-2(1)$ & $1(1)$ & $-1(1)$ \\
\hline$C(7)$ & $16(1)$ & $20(1)$ & $21(1)$ & $1(1)$ & $1(1)$ & $0(1)$ \\
\hline $\mathrm{C}(8)$ & $27(1)$ & $17(1)$ & $23(1)$ & 1(1) & $1(1)$ & $-2(1)$ \\
\hline $\mathrm{C}(9)$ & $26(1)$ & $18(1)$ & $20(1)$ & $1(1)$ & $0(1)$ & 1(1) \\
\hline$C(10)$ & $19(1)$ & $17(1)$ & $18(1)$ & $2(1)$ & $-1(1)$ & $-1(1)$ \\
\hline$C(11)$ & $23(1)$ & $19(1)$ & $17(1)$ & $1(1)$ & $0(1)$ & $0(1)$ \\
\hline $\mathrm{C}(12)$ & $17(1)$ & $19(1)$ & $14(1)$ & $-1(1)$ & $-2(1)$ & $1(1)$ \\
\hline$C(13)$ & $18(1)$ & $18(1)$ & $20(1)$ & $0(1)$ & $0(1)$ & $3(1)$ \\
\hline$C(14)$ & $17(1)$ & $20(1)$ & $15(1)$ & $-2(1)$ & $-2(1)$ & $1(1)$ \\
\hline$C(15)$ & $19(1)$ & $15(1)$ & $18(1)$ & $0(1)$ & $-1(1)$ & $3(1)$ \\
\hline$C(16)$ & $22(1)$ & $18(1)$ & $16(1)$ & $1(1)$ & $-1(1)$ & $1(1)$ \\
\hline$C(17)$ & $23(1)$ & $19(1)$ & $19(1)$ & $-3(1)$ & $0(1)$ & $-1(1)$ \\
\hline$C(18)$ & $52(2)$ & $26(1)$ & $22(1)$ & $6(1)$ & $-3(1)$ & $-10(1)$ \\
\hline$C(19)$ & $25(1)$ & $20(1)$ & $26(1)$ & $-3(1)$ & $-3(1)$ & $-2(1)$ \\
\hline $\mathrm{C}(20)$ & $32(1)$ & $22(1)$ & $20(1)$ & $2(1)$ & $3(1)$ & $-2(1)$ \\
\hline$C(21)$ & $27(1)$ & $22(1)$ & $20(1)$ & $0(1)$ & $-5(1)$ & $3(1)$ \\
\hline $\mathrm{C}(22)$ & $24(1)$ & $31(1)$ & $28(1)$ & $6(1)$ & $7(1)$ & $1(1)$ \\
\hline$C(23)$ & $32(1)$ & $22(1)$ & $17(1)$ & $0(1)$ & $-1(1)$ & $3(1)$ \\
\hline$C(24)$ & $20(1)$ & $30(1)$ & $28(1)$ & $6(1)$ & $1(1)$ & $-4(1)$ \\
\hline
\end{tabular}




\begin{tabular}{|c|c|c|c|c|c|c|}
\hline$C(25)$ & $23(1)$ & $19(1)$ & $19(1)$ & $0(1)$ & $-1(1)$ & $2(1)$ \\
\hline $\mathrm{N}(2)$ & $22(1)$ & $28(1)$ & $30(1)$ & $5(1)$ & $-2(1)$ & $-2(1)$ \\
\hline $\mathrm{O}(7)$ & $29(1)$ & $20(1)$ & $17(1)$ & $0(1)$ & $-1(1)$ & $-1(1)$ \\
\hline $\mathrm{O}(8)$ & $33(1)$ & $15(1)$ & $41(1)$ & $0(1)$ & $9(1)$ & $-3(1)$ \\
\hline $\mathrm{O}(9)$ & $26(1)$ & $16(1)$ & $18(1)$ & $1(1)$ & $0(1)$ & $1(1)$ \\
\hline $\mathrm{O}(10)$ & $21(1)$ & $28(1)$ & $21(1)$ & $0(1)$ & $-5(1)$ & $3(1)$ \\
\hline $\mathrm{O}(11)$ & $22(1)$ & $21(1)$ & $23(1)$ & $3(1)$ & $-2(1)$ & $-3(1)$ \\
\hline $\mathrm{O}(12)$ & $23(1)$ & $14(1)$ & $17(1)$ & $0(1)$ & $1(1)$ & $0(1)$ \\
\hline$C(26)$ & $16(1)$ & $18(1)$ & $19(1)$ & $-1(1)$ & $1(1)$ & $-1(1)$ \\
\hline$C(27)$ & $15(1)$ & $17(1)$ & $22(1)$ & $0(1)$ & $2(1)$ & $-1(1)$ \\
\hline$C(28)$ & $16(1)$ & $20(1)$ & $21(1)$ & $-3(1)$ & $1(1)$ & $1(1)$ \\
\hline$C(29)$ & $21(1)$ & $21(1)$ & $24(1)$ & $3(1)$ & $4(1)$ & $2(1)$ \\
\hline$C(30)$ & $17(1)$ & $16(1)$ & $36(1)$ & $1(1)$ & $6(1)$ & $-1(1)$ \\
\hline $\mathrm{C}(31)$ & $17(1)$ & $21(1)$ & $31(1)$ & $-5(1)$ & $2(1)$ & $0(1)$ \\
\hline$C(32)$ & $13(1)$ & $21(1)$ & $26(1)$ & $-3(1)$ & $1(1)$ & $0(1)$ \\
\hline$C(33)$ & $27(1)$ & $23(1)$ & $23(1)$ & $-5(1)$ & $-1(1)$ & $-1(1)$ \\
\hline$C(34)$ & $27(1)$ & $23(1)$ & $19(1)$ & $-5(1)$ & $1(1)$ & $0(1)$ \\
\hline$C(35)$ & $18(1)$ & $19(1)$ & $18(1)$ & $-1(1)$ & $-2(1)$ & $-1(1)$ \\
\hline$C(36)$ & $24(1)$ & $20(1)$ & $17(1)$ & $0(1)$ & $-2(1)$ & $0(1)$ \\
\hline$C(37)$ & $21(1)$ & $17(1)$ & $19(1)$ & $1(1)$ & $0(1)$ & $0(1)$ \\
\hline C(38) & $24(1)$ & $18(1)$ & $19(1)$ & $0(1)$ & $0(1)$ & $2(1)$ \\
\hline$C(39)$ & $21(1)$ & $15(1)$ & $22(1)$ & $2(1)$ & $-2(1)$ & $-1(1)$ \\
\hline $\mathrm{C}(40)$ & $19(1)$ & $17(1)$ & $16(1)$ & $-1(1)$ & $1(1)$ & $2(1)$ \\
\hline $\mathrm{C}(41)$ & $22(1)$ & $17(1)$ & $17(1)$ & $-1(1)$ & $1(1)$ & $0(1)$ \\
\hline$C(42)$ & $24(1)$ & $17(1)$ & $18(1)$ & $3(1)$ & $-1(1)$ & $-2(1)$ \\
\hline$C(43)$ & $40(1)$ & $32(1)$ & $18(1)$ & $3(1)$ & $-4(1)$ & $-3(1)$ \\
\hline$C(44)$ & $30(1)$ & $18(1)$ & $51(1)$ & $-6(1)$ & 10(1) & $-4(1)$ \\
\hline$C(45)$ & $36(1)$ & $28(1)$ & $19(1)$ & $-2(1)$ & $-7(1)$ & $2(1)$ \\
\hline$C(46)$ & $29(1)$ & $24(1)$ & $21(1)$ & $2(1)$ & $4(1)$ & $0(1)$ \\
\hline$C(47)$ & $28(1)$ & $44(1)$ & $28(1)$ & $2(1)$ & $-9(1)$ & $7(1)$ \\
\hline$C(48)$ & $36(1)$ & $19(1)$ & $25(1)$ & $3(1)$ & $-3(1)$ & $0(1)$ \\
\hline C(49) & $25(1)$ & $32(1)$ & $28(1)$ & $4(1)$ & $-9(1)$ & $-4(1)$ \\
\hline$C(50)$ & $29(1)$ & $19(1)$ & 21(1) & $-2(1)$ & $-1(1)$ & $3(1)$ \\
\hline
\end{tabular}


Table 5. Hydrogen coordinates $\left(\times 10^{4}\right)$ and isotropic displacement parameters $\left(\AA^{2} \times 10^{3}\right)$ for cdv87.

\begin{tabular}{|c|c|c|c|c|}
\hline & $\mathrm{x}$ & $\mathrm{y}$ & z & $\mathrm{U}(\mathrm{eq})$ \\
\hline $\mathrm{H}(4 \mathrm{~A})$ & 7078 & 3769 & 4557 & 24 \\
\hline $\mathrm{H}(6 \mathrm{~A})$ & 8308 & 5782 & 4034 & 23 \\
\hline $\mathrm{H}(8 \mathrm{~A})$ & 9191 & 5324 & 3397 & 27 \\
\hline $\mathrm{H}(8 \mathrm{~B})$ & 7264 & 5810 & 3471 & 27 \\
\hline $\mathrm{H}(9 \mathrm{~A})$ & 7245 & 5124 & 2953 & 26 \\
\hline $\mathrm{H}(9 \mathrm{~B})$ & 5466 & 4880 & 3178 & 26 \\
\hline $\mathrm{H}(10 \mathrm{~A})$ & 8910 & 3976 & 3195 & 22 \\
\hline $\mathrm{H}(12 \mathrm{~A})$ & 9377 & 2666 & 2894 & 20 \\
\hline $\mathrm{H}(15 \mathrm{~A})$ & 5567 & 2230 & 3074 & 21 \\
\hline $\mathrm{H}(16 \mathrm{~A})$ & 8781 & 2621 & 3477 & 22 \\
\hline $\mathrm{H}(16 \mathrm{~B})$ & 6780 & 2297 & 3613 & 22 \\
\hline $\mathrm{H}(18 \mathrm{~A})$ & 5900 & 1825 & 4347 & 50 \\
\hline $\mathrm{H}(18 \mathrm{~B})$ & 5400 & 2653 & 4534 & 50 \\
\hline $\mathrm{H}(18 \mathrm{C})$ & 7577 & 2414 & 4467 & 50 \\
\hline $\mathrm{H}(19 \mathrm{~A})$ & 8793 & 6212 & 4862 & 35 \\
\hline $\mathrm{H}(19 \mathrm{~B})$ & 7544 & 6340 & 4542 & 35 \\
\hline $\mathrm{H}(19 \mathrm{C})$ & 9768 & 6134 & 4513 & 35 \\
\hline $\mathrm{H}(20 \mathrm{~A})$ & 9994 & 4002 & 2682 & 37 \\
\hline $\mathrm{H}(20 \mathrm{~B})$ & 8286 & 4529 & 2534 & 37 \\
\hline $\mathrm{H}(20 \mathrm{C})$ & 8822 & 3668 & 2377 & 37 \\
\hline $\mathrm{H}(21 \mathrm{~A})$ & 5308 & 3371 & 2446 & 35 \\
\hline $\mathrm{H}(21 \mathrm{~B})$ & 4934 & 4186 & 2649 & 35 \\
\hline $\mathrm{H}(21 \mathrm{C})$ & 4402 & 3321 & 2802 & 35 \\
\hline $\mathrm{H}(22 \mathrm{~A})$ & 12784 & 1813 & 2474 & 42 \\
\hline $\mathrm{H}(22 \mathrm{~B})$ & 11016 & 2374 & 2374 & 42 \\
\hline $\mathrm{H}(22 \mathrm{C})$ & 11447 & 1555 & 2178 & 42 \\
\hline $\mathrm{H}(23 \mathrm{~A})$ & 8343 & 1386 & 2050 & 35 \\
\hline $\mathrm{H}(23 \mathrm{~B})$ & 6352 & 1087 & 2198 & 35 \\
\hline $\mathrm{H}(23 \mathrm{C})$ & 8174 & 501 & 2211 & 35 \\
\hline $\mathrm{H}(24 \mathrm{~A})$ & 2832 & 766 & 2958 & 39 \\
\hline
\end{tabular}




\begin{tabular}{|c|c|c|c|c|}
\hline $\mathrm{H}(24 \mathrm{~B})$ & 4560 & 746 & 3211 & 39 \\
\hline $\mathrm{H}(24 \mathrm{C})$ & 4215 & -8 & 2973 & 39 \\
\hline $\mathrm{H}(25 \mathrm{~A})$ & 7525 & -114 & 3089 & 31 \\
\hline $\mathrm{H}(25 \mathrm{~B})$ & 9515 & 161 & 2935 & 31 \\
\hline $\mathrm{H}(25 \mathrm{C})$ & 7891 & -223 & 2709 & 31 \\
\hline $\mathrm{H}(29 \mathrm{~A})$ & 3501 & 5309 & 4862 & 26 \\
\hline $\mathrm{H}(31 \mathrm{~A})$ & 2448 & 4746 & 3924 & 27 \\
\hline $\mathrm{H}(33 \mathrm{~A})$ & 1630 & 6132 & 3573 & 29 \\
\hline $\mathrm{H}(33 \mathrm{~B})$ & 3659 & 5712 & 3517 & 29 \\
\hline $\mathrm{H}(34 \mathrm{~A})$ & 3515 & 7106 & 3329 & 28 \\
\hline $\mathrm{H}(34 \mathrm{~B})$ & 5238 & 6943 & 3577 & 28 \\
\hline $\mathrm{H}(35 \mathrm{~A})$ & 1621 & 7457 & 3805 & 22 \\
\hline $\mathrm{H}(37 \mathrm{~A})$ & 862 & 8919 & 3946 & 22 \\
\hline $\mathrm{H}(40 \mathrm{~A})$ & 4603 & 9023 & 4221 & 21 \\
\hline $\mathrm{H}(41 \mathrm{~A})$ & 1507 & 7947 & 4378 & 22 \\
\hline $\mathrm{H}(41 \mathrm{~B})$ & 3436 & 8003 & 4585 & 22 \\
\hline $\mathrm{H}(43 \mathrm{~A})$ & 4127 & 7163 & 5240 & 44 \\
\hline $\mathrm{H}(43 \mathrm{~B})$ & 4773 & 6239 & 5184 & 44 \\
\hline $\mathrm{H}(43 \mathrm{C})$ & 2588 & 6492 & 5137 & 44 \\
\hline $\mathrm{H}(44 \mathrm{~A})$ & 1636 & 3029 & 4393 & 49 \\
\hline $\mathrm{H}(44 \mathrm{~B})$ & 2997 & 3451 & 4131 & 49 \\
\hline $\mathrm{H}(44 \mathrm{C})$ & 825 & 3737 & 4165 & 49 \\
\hline $\mathrm{H}(45 \mathrm{~A})$ & 1591 & 9110 & 3298 & 42 \\
\hline $\mathrm{H}(45 \mathrm{~B})$ & 474 & 8319 & 3421 & 42 \\
\hline $\mathrm{H}(45 \mathrm{C})$ & 2260 & 8226 & 3182 & 42 \\
\hline $\mathrm{H}(46 \mathrm{~A})$ & 5028 & 9318 & 3478 & 37 \\
\hline $\mathrm{H}(46 \mathrm{~B})$ & 5521 & 8402 & 3376 & 37 \\
\hline $\mathrm{H}(46 \mathrm{C})$ & 5947 & 8708 & 3736 & 37 \\
\hline $\mathrm{H}(47 \mathrm{~A})$ & -2748 & 10287 & 3885 & 50 \\
\hline $\mathrm{H}(47 \mathrm{~B})$ & -1012 & 9996 & 3661 & 50 \\
\hline $\mathrm{H}(47 \mathrm{C})$ & -1329 & 10945 & 3731 & 50 \\
\hline $\mathrm{H}(48 \mathrm{~A})$ & 1701 & 11316 & 3687 & 40 \\
\hline $\mathrm{H}(48 \mathrm{~B})$ & 3612 & 11341 & 3899 & 40 \\
\hline $\mathrm{H}(48 \mathrm{C})$ & 1673 & 11704 & 4043 & 40 \\
\hline $\mathrm{H}(49 \mathrm{~A})$ & 7122 & 10322 & 4565 & 42 \\
\hline $\mathrm{H}(49 \mathrm{~B})$ & 5441 & 9791 & 4720 & 42 \\
\hline
\end{tabular}




\begin{tabular}{lrlll}
$\mathrm{H}(49 \mathrm{C})$ & 5560 & 10748 & 4790 & 42 \\
$\mathrm{H}(50 \mathrm{~A})$ & 2240 & 10619 & 4867 & 35 \\
$\mathrm{H}(50 \mathrm{~B})$ & 347 & 10709 & 4654 & 35 \\
$\mathrm{H}(50 \mathrm{C})$ & 2011 & 11372 & 4624 & 35 \\
\hline
\end{tabular}


Table 6. Torsion angles $\left[{ }^{\circ}\right]$ for $\operatorname{cdv} 87$.

\begin{tabular}{|c|c|}
\hline $\mathrm{C}(17)-\mathrm{C}(1)-\mathrm{C}(2)-\mathrm{C}(7)$ & $-100.3(2)$ \\
\hline $\mathrm{C}(16)-\mathrm{C}(1)-\mathrm{C}(2)-\mathrm{C}(7)$ & $140.29(18)$ \\
\hline $\mathrm{C}(10)-\mathrm{C}(1)-\mathrm{C}(2)-\mathrm{C}(7)$ & $19.6(2)$ \\
\hline $\mathrm{C}(17)-\mathrm{C}(1)-\mathrm{C}(2)-\mathrm{C}(3)$ & $75.7(2)$ \\
\hline$C(16)-C(1)-C(2)-C(3)$ & $-43.6(2)$ \\
\hline $\mathrm{C}(10)-\mathrm{C}(1)-\mathrm{C}(2)-\mathrm{C}(3)$ & $-164.31(17)$ \\
\hline $\mathrm{C}(18)-\mathrm{O}(1)-\mathrm{C}(3)-\mathrm{C}(4)$ & $-11.3(3)$ \\
\hline $\mathrm{C}(18)-\mathrm{O}(1)-\mathrm{C}(3)-\mathrm{C}(2)$ & $170.02(18)$ \\
\hline $\mathrm{C}(7)-\mathrm{C}(2)-\mathrm{C}(3)-\mathrm{O}(1)$ & $175.77(17)$ \\
\hline $\mathrm{C}(1)-\mathrm{C}(2)-\mathrm{C}(3)-\mathrm{O}(1)$ & $-0.5(3)$ \\
\hline$C(7)-C(2)-C(3)-C(4)$ & $-3.0(3)$ \\
\hline $\mathrm{C}(1)-\mathrm{C}(2)-\mathrm{C}(3)-\mathrm{C}(4)$ & $-179.22(18)$ \\
\hline $\mathrm{O}(1)-\mathrm{C}(3)-\mathrm{C}(4)-\mathrm{C}(5)$ & $-177.82(18)$ \\
\hline $\mathrm{C}(2)-\mathrm{C}(3)-\mathrm{C}(4)-\mathrm{C}(5)$ & $0.8(3)$ \\
\hline $\mathrm{C}(19)-\mathrm{O}(2)-\mathrm{C}(5)-\mathrm{C}(6)$ & $5.5(3)$ \\
\hline $\mathrm{C}(19)-\mathrm{O}(2)-\mathrm{C}(5)-\mathrm{C}(4)$ & $-174.42(17)$ \\
\hline $\mathrm{C}(3)-\mathrm{C}(4)-\mathrm{C}(5)-\mathrm{O}(2)$ & $-178.35(17)$ \\
\hline$C(3)-C(4)-C(5)-C(6)$ & $1.7(3)$ \\
\hline $\mathrm{O}(2)-\mathrm{C}(5)-\mathrm{C}(6)-\mathrm{C}(7)$ & $178.05(18)$ \\
\hline$C(4)-C(5)-C(6)-C(7)$ & $-2.0(3)$ \\
\hline$C(3)-C(2)-C(7)-C(6)$ & $2.6(3)$ \\
\hline $\mathrm{C}(1)-\mathrm{C}(2)-\mathrm{C}(7)-\mathrm{C}(6)$ & $178.92(17)$ \\
\hline$C(3)-C(2)-C(7)-C(8)$ & $-178.60(18)$ \\
\hline $\mathrm{C}(1)-\mathrm{C}(2)-\mathrm{C}(7)-\mathrm{C}(8)$ & $-2.3(3)$ \\
\hline$C(5)-C(6)-C(7)-C(2)$ & $-0.2(3)$ \\
\hline$C(5)-C(6)-C(7)-C(8)$ & $-179.07(18)$ \\
\hline $\mathrm{C}(2)-\mathrm{C}(7)-\mathrm{C}(8)-\mathrm{C}(9)$ & $17.7(3)$ \\
\hline $\mathrm{C}(6)-\mathrm{C}(7)-\mathrm{C}(8)-\mathrm{C}(9)$ & $-163.46(17)$ \\
\hline$C(7)-C(8)-C(9)-C(10)$ & $-50.1(2)$ \\
\hline $\mathrm{C}(8)-\mathrm{C}(9)-\mathrm{C}(10)-\mathrm{C}(11)$ & $-157.84(16)$ \\
\hline$C(8)-C(9)-C(10)-C(1)$ & $69.1(2)$ \\
\hline $\mathrm{C}(17)-\mathrm{C}(1)-\mathrm{C}(10)-\mathrm{C}(9)$ & $63.9(2)$ \\
\hline$C(16)-C(1)-C(10)-C(9)$ & $-177.14(15)$ \\
\hline$C(2)-C(1)-C(10)-C(9)$ & $-52.3(2)$ \\
\hline
\end{tabular}




\begin{tabular}{|c|c|}
\hline$C(17)-C(1)-C(10)-C(11)$ & $-67.4(2)$ \\
\hline $\mathrm{C}(16)-\mathrm{C}(1)-\mathrm{C}(10)-\mathrm{C}(11)$ & $51.6(2)$ \\
\hline$C(2)-C(1)-C(10)-C(11)$ & $176.40(16)$ \\
\hline $\mathrm{C}(9)-\mathrm{C}(10)-\mathrm{C}(11)-\mathrm{C}(12)$ & $-178.95(16)$ \\
\hline$C(1)-C(10)-C(11)-C(12)$ & $-49.8(2)$ \\
\hline $\mathrm{C}(9)-\mathrm{C}(10)-\mathrm{C}(11)-\mathrm{C}(20)$ & $65.9(2)$ \\
\hline$C(1)-C(10)-C(11)-C(20)$ & $-164.88(16)$ \\
\hline $\mathrm{C}(9)-\mathrm{C}(10)-\mathrm{C}(11)-\mathrm{C}(21)$ & $-54.4(2)$ \\
\hline$C(1)-C(10)-C(11)-C(21)$ & $74.8(2)$ \\
\hline $\mathrm{C}(13)-\mathrm{O}(3)-\mathrm{C}(12)-\mathrm{C}(15)$ & $-57.07(19)$ \\
\hline $\mathrm{C}(13)-\mathrm{O}(3)-\mathrm{C}(12)-\mathrm{C}(11)$ & $178.85(15)$ \\
\hline $\mathrm{C}(20)-\mathrm{C}(11)-\mathrm{C}(12)-\mathrm{O}(3)$ & $-69.17(19)$ \\
\hline $\mathrm{C}(21)-\mathrm{C}(11)-\mathrm{C}(12)-\mathrm{O}(3)$ & $49.7(2)$ \\
\hline $\mathrm{C}(10)-\mathrm{C}(11)-\mathrm{C}(12)-\mathrm{O}(3)$ & $175.32(15)$ \\
\hline$C(20)-C(11)-C(12)-C(15)$ & $168.35(15)$ \\
\hline $\mathrm{C}(21)-\mathrm{C}(11)-\mathrm{C}(12)-\mathrm{C}(15)$ & $-72.74(19)$ \\
\hline$C(10)-C(11)-C(12)-C(15)$ & $52.8(2)$ \\
\hline $\mathrm{C}(22)-\mathrm{O}(4)-\mathrm{C}(13)-\mathrm{O}(3)$ & $-55.1(2)$ \\
\hline $\mathrm{C}(22)-\mathrm{O}(4)-\mathrm{C}(13)-\mathrm{C}(23)$ & $63.0(2)$ \\
\hline $\mathrm{C}(22)-\mathrm{O}(4)-\mathrm{C}(13)-\mathrm{C}(14)$ & $-174.30(15)$ \\
\hline $\mathrm{C}(12)-\mathrm{O}(3)-\mathrm{C}(13)-\mathrm{O}(4)$ & $-61.94(19)$ \\
\hline $\mathrm{C}(12)-\mathrm{O}(3)-\mathrm{C}(13)-\mathrm{C}(23)$ & $176.21(15)$ \\
\hline $\mathrm{C}(12)-\mathrm{O}(3)-\mathrm{C}(13)-\mathrm{C}(14)$ & $53.96(19)$ \\
\hline $\mathrm{C}(24)-\mathrm{O}(5)-\mathrm{C}(14)-\mathrm{O}(6)$ & $-59.7(2)$ \\
\hline $\mathrm{C}(24)-\mathrm{O}(5)-\mathrm{C}(14)-\mathrm{C}(25)$ & $57.8(2)$ \\
\hline $\mathrm{C}(24)-\mathrm{O}(5)-\mathrm{C}(14)-\mathrm{C}(13)$ & $-177.93(15)$ \\
\hline $\mathrm{C}(15)-\mathrm{O}(6)-\mathrm{C}(14)-\mathrm{O}(5)$ & $-58.70(19)$ \\
\hline $\mathrm{C}(15)-\mathrm{O}(6)-\mathrm{C}(14)-\mathrm{C}(25)$ & $179.25(15)$ \\
\hline $\mathrm{C}(15)-\mathrm{O}(6)-\mathrm{C}(14)-\mathrm{C}(13)$ & $55.83(19)$ \\
\hline $\mathrm{O}(4)-\mathrm{C}(13)-\mathrm{C}(14)-\mathrm{O}(5)$ & $-174.29(14)$ \\
\hline $\mathrm{O}(3)-\mathrm{C}(13)-\mathrm{C}(14)-\mathrm{O}(5)$ & $66.14(18)$ \\
\hline $\mathrm{C}(23)-\mathrm{C}(13)-\mathrm{C}(14)-\mathrm{O}(5)$ & $-52.0(2)$ \\
\hline $\mathrm{O}(4)-\mathrm{C}(13)-\mathrm{C}(14)-\mathrm{O}(6)$ & $67.29(18)$ \\
\hline $\mathrm{O}(3)-\mathrm{C}(13)-\mathrm{C}(14)-\mathrm{O}(6)$ & $-52.28(19)$ \\
\hline $\mathrm{C}(23)-\mathrm{C}(13)-\mathrm{C}(14)-\mathrm{O}(6)$ & $-170.40(15)$ \\
\hline $\mathrm{O}(4)-\mathrm{C}(13)-\mathrm{C}(14)-\mathrm{C}(25)$ & $-50.9(2)$ \\
\hline
\end{tabular}




\begin{tabular}{|c|c|}
\hline $\mathrm{O}(3)-\mathrm{C}(13)-\mathrm{C}(14)-\mathrm{C}(25)$ & $-170.44(15)$ \\
\hline$C(23)-C(13)-C(14)-C(25)$ & $71.4(2)$ \\
\hline $\mathrm{C}(14)-\mathrm{O}(6)-\mathrm{C}(15)-\mathrm{C}(12)$ & $-58.92(19)$ \\
\hline $\mathrm{C}(14)-\mathrm{O}(6)-\mathrm{C}(15)-\mathrm{C}(16)$ & $-179.56(15)$ \\
\hline $\mathrm{O}(3)-\mathrm{C}(12)-\mathrm{C}(15)-\mathrm{O}(6)$ & $57.31(19)$ \\
\hline $\mathrm{C}(11)-\mathrm{C}(12)-\mathrm{C}(15)-\mathrm{O}(6)$ & $179.26(14)$ \\
\hline $\mathrm{O}(3)-\mathrm{C}(12)-\mathrm{C}(15)-\mathrm{C}(16)$ & $175.69(15)$ \\
\hline $\mathrm{C}(11)-\mathrm{C}(12)-\mathrm{C}(15)-\mathrm{C}(16)$ & $-62.4(2)$ \\
\hline $\mathrm{O}(6)-\mathrm{C}(15)-\mathrm{C}(16)-\mathrm{C}(1)$ & $-177.23(15)$ \\
\hline $\mathrm{C}(12)-\mathrm{C}(15)-\mathrm{C}(16)-\mathrm{C}(1)$ & $62.9(2)$ \\
\hline$C(17)-C(1)-C(16)-C(15)$ & $65.4(2)$ \\
\hline$C(2)-C(1)-C(16)-C(15)$ & $-177.17(15)$ \\
\hline$C(10)-C(1)-C(16)-C(15)$ & $-55.3(2)$ \\
\hline$C(42)-C(26)-C(27)-C(32)$ & $-101.7(2)$ \\
\hline$C(41)-C(26)-C(27)-C(32)$ & $137.78(18)$ \\
\hline$C(35)-C(26)-C(27)-C(32)$ & $18.0(2)$ \\
\hline $\mathrm{C}(42)-\mathrm{C}(26)-\mathrm{C}(27)-\mathrm{C}(28)$ & $75.9(2)$ \\
\hline $\mathrm{C}(41)-\mathrm{C}(26)-\mathrm{C}(27)-\mathrm{C}(28)$ & $-44.6(2)$ \\
\hline$C(35)-C(26)-C(27)-C(28)$ & $-164.40(16)$ \\
\hline $\mathrm{C}(43)-\mathrm{O}(7)-\mathrm{C}(28)-\mathrm{C}(29)$ & $-19.9(3)$ \\
\hline $\mathrm{C}(43)-\mathrm{O}(7)-\mathrm{C}(28)-\mathrm{C}(27)$ & $161.22(18)$ \\
\hline $\mathrm{C}(32)-\mathrm{C}(27)-\mathrm{C}(28)-\mathrm{O}(7)$ & $174.93(16)$ \\
\hline $\mathrm{C}(26)-\mathrm{C}(27)-\mathrm{C}(28)-\mathrm{O}(7)$ & $-2.8(3)$ \\
\hline $\mathrm{C}(32)-\mathrm{C}(27)-\mathrm{C}(28)-\mathrm{C}(29)$ & $-4.0(3)$ \\
\hline$C(26)-C(27)-C(28)-C(29)$ & $178.34(18)$ \\
\hline $\mathrm{O}(7)-\mathrm{C}(28)-\mathrm{C}(29)-\mathrm{C}(30)$ & $-178.30(17)$ \\
\hline $\mathrm{C}(27)-\mathrm{C}(28)-\mathrm{C}(29)-\mathrm{C}(30)$ & $0.5(3)$ \\
\hline $\mathrm{C}(44)-\mathrm{O}(8)-\mathrm{C}(30)-\mathrm{C}(31)$ & $-1.5(3)$ \\
\hline $\mathrm{C}(44)-\mathrm{O}(8)-\mathrm{C}(30)-\mathrm{C}(29)$ & $179.31(17)$ \\
\hline $\mathrm{C}(28)-\mathrm{C}(29)-\mathrm{C}(30)-\mathrm{O}(8)$ & $-178.08(18)$ \\
\hline $\mathrm{C}(28)-\mathrm{C}(29)-\mathrm{C}(30)-\mathrm{C}(31)$ & $2.6(3)$ \\
\hline $\mathrm{O}(8)-\mathrm{C}(30)-\mathrm{C}(31)-\mathrm{C}(32)$ & $178.58(18)$ \\
\hline $\mathrm{C}(29)-\mathrm{C}(30)-\mathrm{C}(31)-\mathrm{C}(32)$ & $-2.2(3)$ \\
\hline $\mathrm{C}(30)-\mathrm{C}(31)-\mathrm{C}(32)-\mathrm{C}(27)$ & $-1.4(3)$ \\
\hline $\mathrm{C}(30)-\mathrm{C}(31)-\mathrm{C}(32)-\mathrm{C}(33)$ & $179.37(18)$ \\
\hline$C(28)-C(27)-C(32)-C(31)$ & $4.4(3)$ \\
\hline
\end{tabular}




\begin{tabular}{|c|c|}
\hline$C(26)-C(27)-C(32)-C(31)$ & $-177.92(17)$ \\
\hline$C(28)-C(27)-C(32)-C(33)$ & $-176.44(17)$ \\
\hline$C(26)-C(27)-C(32)-C(33)$ & $1.3(3)$ \\
\hline $\mathrm{C}(31)-\mathrm{C}(32)-\mathrm{C}(33)-\mathrm{C}(34)$ & $-166.12(18)$ \\
\hline$C(27)-C(32)-C(33)-C(34)$ & $14.7(3)$ \\
\hline $\mathrm{C}(32)-\mathrm{C}(33)-\mathrm{C}(34)-\mathrm{C}(35)$ & $-49.5(2)$ \\
\hline$C(33)-C(34)-C(35)-C(36)$ & $-157.07(16)$ \\
\hline$C(33)-C(34)-C(35)-C(26)$ & $70.3(2)$ \\
\hline$C(42)-C(26)-C(35)-C(34)$ & $63.6(2)$ \\
\hline$C(41)-C(26)-C(35)-C(34)$ & $-176.84(15)$ \\
\hline$C(27)-C(26)-C(35)-C(34)$ & $-52.90(19)$ \\
\hline$C(42)-C(26)-C(35)-C(36)$ & $-67.4(2)$ \\
\hline$C(41)-C(26)-C(35)-C(36)$ & $52.1(2)$ \\
\hline$C(27)-C(26)-C(35)-C(36)$ & $176.00(16)$ \\
\hline$C(34)-C(35)-C(36)-C(46)$ & $-54.3(2)$ \\
\hline $\mathrm{C}(26)-\mathrm{C}(35)-\mathrm{C}(36)-\mathrm{C}(46)$ & $74.2(2)$ \\
\hline$C(34)-C(35)-C(36)-C(37)$ & $-178.18(16)$ \\
\hline$C(26)-C(35)-C(36)-C(37)$ & $-49.6(2)$ \\
\hline $\mathrm{C}(34)-\mathrm{C}(35)-\mathrm{C}(36)-\mathrm{C}(45)$ & $66.3(2)$ \\
\hline$C(26)-C(35)-C(36)-C(45)$ & $-165.17(16)$ \\
\hline $\mathrm{C}(38)-\mathrm{O}(9)-\mathrm{C}(37)-\mathrm{C}(40)$ & $-56.3(2)$ \\
\hline $\mathrm{C}(38)-\mathrm{O}(9)-\mathrm{C}(37)-\mathrm{C}(36)$ & $-179.91(15)$ \\
\hline $\mathrm{C}(46)-\mathrm{C}(36)-\mathrm{C}(37)-\mathrm{O}(9)$ & $49.9(2)$ \\
\hline $\mathrm{C}(45)-\mathrm{C}(36)-\mathrm{C}(37)-\mathrm{O}(9)$ & $-69.06(19)$ \\
\hline $\mathrm{C}(35)-\mathrm{C}(36)-\mathrm{C}(37)-\mathrm{O}(9)$ & $174.69(15)$ \\
\hline $\mathrm{C}(46)-\mathrm{C}(36)-\mathrm{C}(37)-\mathrm{C}(40)$ & $-72.3(2)$ \\
\hline $\mathrm{C}(45)-\mathrm{C}(36)-\mathrm{C}(37)-\mathrm{C}(40)$ & $168.82(16)$ \\
\hline $\mathrm{C}(35)-\mathrm{C}(36)-\mathrm{C}(37)-\mathrm{C}(40)$ & $52.6(2)$ \\
\hline $\mathrm{C}(47)-\mathrm{O}(10)-\mathrm{C}(38)-\mathrm{O}(9)$ & $-57.8(2)$ \\
\hline $\mathrm{C}(47)-\mathrm{O}(10)-\mathrm{C}(38)-\mathrm{C}(48)$ & $60.1(2)$ \\
\hline $\mathrm{C}(47)-\mathrm{O}(10)-\mathrm{C}(38)-\mathrm{C}(39)$ & $-176.58(16)$ \\
\hline $\mathrm{C}(37)-\mathrm{O}(9)-\mathrm{C}(38)-\mathrm{O}(10)$ & $-61.36(19)$ \\
\hline $\mathrm{C}(37)-\mathrm{O}(9)-\mathrm{C}(38)-\mathrm{C}(48)$ & $175.95(16)$ \\
\hline $\mathrm{C}(37)-\mathrm{O}(9)-\mathrm{C}(38)-\mathrm{C}(39)$ & $53.4(2)$ \\
\hline $\mathrm{C}(49)-\mathrm{O}(11)-\mathrm{C}(39)-\mathrm{O}(12)$ & $-57.4(2)$ \\
\hline $\mathrm{C}(49)-\mathrm{O}(11)-\mathrm{C}(39)-\mathrm{C}(50)$ & $60.7(2)$ \\
\hline
\end{tabular}




\begin{tabular}{lc}
$\mathrm{C}(49)-\mathrm{O}(11)-\mathrm{C}(39)-\mathrm{C}(38)$ & $-175.48(15)$ \\
$\mathrm{C}(40)-\mathrm{O}(12)-\mathrm{C}(39)-\mathrm{O}(11)$ & $-58.29(19)$ \\
$\mathrm{C}(40)-\mathrm{O}(12)-\mathrm{C}(39)-\mathrm{C}(50)$ & $179.74(15)$ \\
$\mathrm{C}(40)-\mathrm{O}(12)-\mathrm{C}(39)-\mathrm{C}(38)$ & $56.9(2)$ \\
$\mathrm{O}(10)-\mathrm{C}(38)-\mathrm{C}(39)-\mathrm{O}(11)$ & $-174.58(14)$ \\
$\mathrm{O}(9)-\mathrm{C}(38)-\mathrm{C}(39)-\mathrm{O}(11)$ & $65.94(18)$ \\
$\mathrm{C}(48)-\mathrm{C}(38)-\mathrm{C}(39)-\mathrm{O}(11)$ & $-51.5(2)$ \\
$\mathrm{O}(10)-\mathrm{C}(38)-\mathrm{C}(39)-\mathrm{O}(12)$ & $66.90(18)$ \\
$\mathrm{O}(9)-\mathrm{C}(38)-\mathrm{C}(39)-\mathrm{O}(12)$ & $-52.6(2)$ \\
$\mathrm{C}(48)-\mathrm{C}(38)-\mathrm{C}(39)-\mathrm{O}(12)$ & $-169.98(16)$ \\
$\mathrm{O}(10)-\mathrm{C}(38)-\mathrm{C}(39)-\mathrm{C}(50)$ & $-51.4(2)$ \\
$\mathrm{O}(9)-\mathrm{C}(38)-\mathrm{C}(39)-\mathrm{C}(50)$ & $-170.85(16)$ \\
$\mathrm{C}(48)-\mathrm{C}(38)-\mathrm{C}(39)-\mathrm{C}(50)$ & $71.7(2)$ \\
$\mathrm{C}(39)-\mathrm{O}(12)-\mathrm{C}(40)-\mathrm{C}(37)$ & $-60.15(19)$ \\
$\mathrm{C}(39)-\mathrm{O}(12)-\mathrm{C}(40)-\mathrm{C}(41)$ & $179.27(15)$ \\
$\mathrm{O}(9)-\mathrm{C}(37)-\mathrm{C}(40)-\mathrm{O}(12)$ & $57.49(19)$ \\
$\mathrm{C}(36)-\mathrm{C}(37)-\mathrm{C}(40)-\mathrm{O}(12)$ & $178.94(15)$ \\
$\mathrm{O}(9)-\mathrm{C}(37)-\mathrm{C}(40)-\mathrm{C}(41)$ & $176.18(15)$ \\
$\mathrm{C}(36)-\mathrm{C}(37)-\mathrm{C}(40)-\mathrm{C}(41)$ & $-62.4(2)$ \\
$\mathrm{O}(12)-\mathrm{C}(40)-\mathrm{C}(41)-\mathrm{C}(26)$ & $-176.00(15)$ \\
$\mathrm{C}(37)-\mathrm{C}(40)-\mathrm{C}(41)-\mathrm{C}(26)$ & $64.0(2)$ \\
$\mathrm{C}(42)-\mathrm{C}(26)-\mathrm{C}(41)-\mathrm{C}(40)$ & $-177.40(15)$ \\
$\mathrm{C}(27)-\mathrm{C}(26)-\mathrm{C}(41)-\mathrm{C}(40)$ & $-56.4(2)$ \\
$\mathrm{C}(35)-\mathrm{C}(26)-\mathrm{C}(41)-\mathrm{C}(40)$ & \\
& \\
\hline & \\
& \\
& \\
&
\end{tabular}


X-ray Data Collection, Structure Solution and Refinement for diol 22b (cdv86).

A colorless crystal of approximate dimensions $0.122 \times 0.267 \times 0.288 \mathrm{~mm}$ was mounted in a cryoloop and transferred to a Bruker Prospector APEX II diffractometer system. The APEX $3^{1}$ program package was used to determine the unit-cell parameters and for data collection (4 sec/frame scan time). The raw frame data was processed using SAINT ${ }^{2}$ and $\mathrm{SADABS}^{3}$ to yield the reflection data file. Subsequent calculations were carried out using the SHELXTL ${ }^{4}$ program package. The diffraction symmetry was $\mathrm{mmm}$ and the systematic absences were consistent with the orthorhombic space group $P 2{ }_{1} 2_{12}{ }_{1}$ that was later determined to be correct.

The structure was solved by direct methods and refined on $\mathrm{F}^{2}$ by full-matrix least-squares techniques. The analytical scattering factors 5 for neutral atoms were used throughout the analysis. Hydrogen atoms $\mathrm{H}(3)$ and $\mathrm{H}(4)$ were located from a difference-Fourier map and refined $\left(x, y, z\right.$ and $\left.U_{\text {iso }}\right)$. The remaining hydrogen atoms were included using a riding model.

Least squares analysis yielded $w R 2=0.0931$ and Goof $=1.078$ for 231 variables refined against 3117 data $(0.83 \AA), R 1=0.0364$ for those 2959 data with $I>2.0 \sigma(I)$. The structure was refined as an inversion twin. The absolute structure assignment was based on the synthetic method employed.

References.

1. APEX3 Version 2017.3-0, Bruker AXS, Inc.; Madison, WI 2017.

2. SAINT Version 8.38a, Bruker AXS, Inc.; Madison, WI 2013.

3. Sheldrick, G. M. SADABS, Version 2014/5, Bruker AXS, Inc.; Madison, WI 2014.

4. Sheldrick, G. M. SHELXTL, Version 2014/7, Bruker AXS, Inc.; Madison, WI 2014.

5. International Tables for Crystallography 1992, Vol. C., Dordrecht: Kluwer Academic Publishers. 


\section{Definitions:}

$w R 2=\left[\Sigma\left[w\left(F_{o}^{2}-F_{c}^{2}\right)^{2}\right] / \Sigma\left[w\left(F_{o}^{2}\right)^{2}\right]\right]^{1 / 2}$

$\mathrm{R} 1=\Sigma|| \mathrm{F}_{\mathrm{o}} \mathrm{l}-\mathrm{I} \mathrm{F}_{\mathrm{c}} \mid / \Sigma \mathrm{F}_{\mathrm{o}} \mathrm{l}$

Goof $=S=\left[\Sigma\left[w\left(F_{o}^{2}-F_{c}^{2}\right)^{2}\right] /(n-p)\right]^{1 / 2}$ where $n$ is the number of reflections and $p$ is the total number of parameters refined.

The thermal ellipsoid plot is shown at the $50 \%$ probability level.

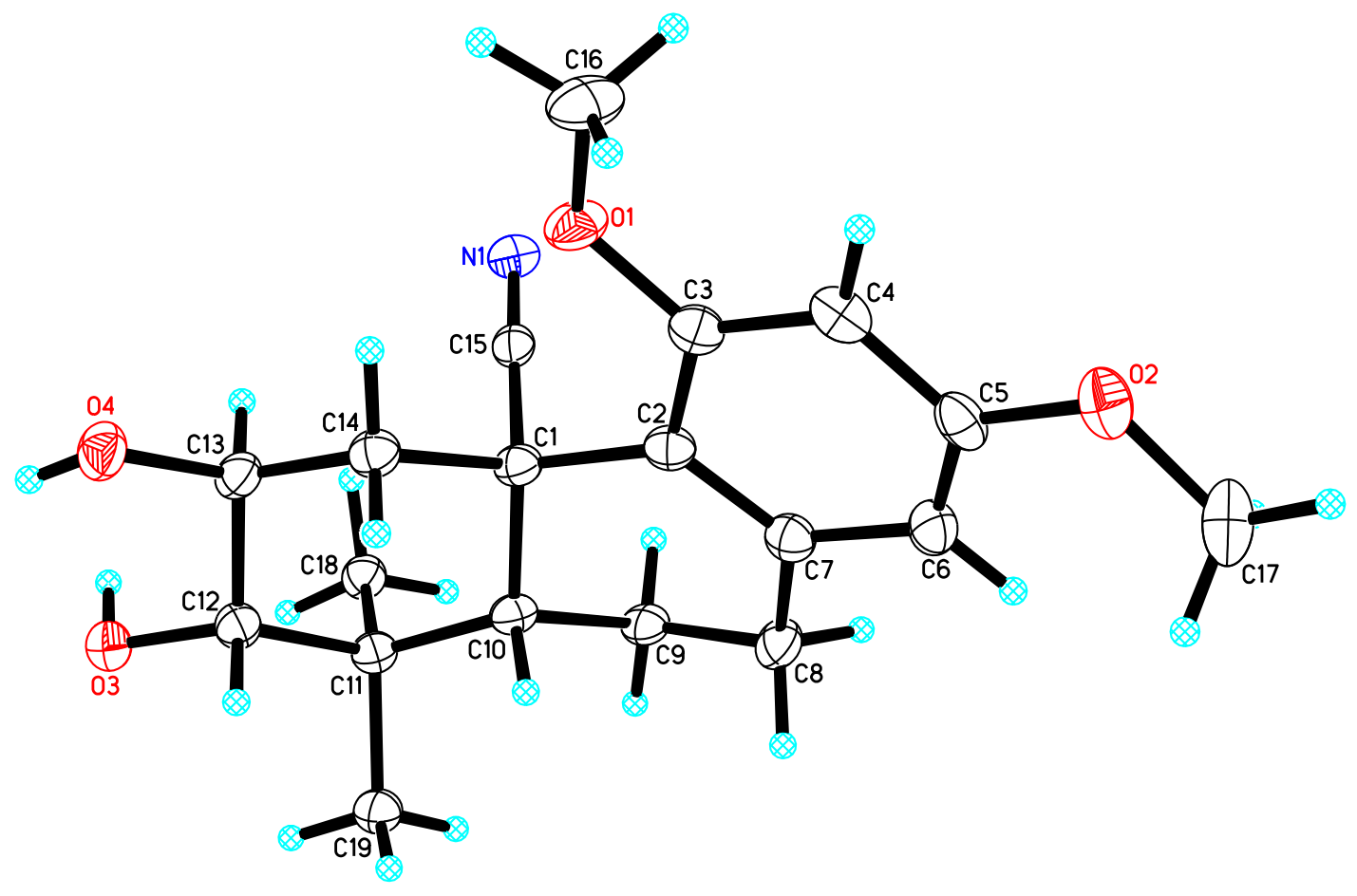


Table 1. Crystal data and structure refinement for cdv86.

Identification code

Empirical formula

Formula weight

Temperature

Wavelength

Crystal system

Space group

Unit cell dimensions

Volume

Z

Density (calculated)

Absorption coefficient

$\mathrm{F}(000)$

Crystal color

Crystal size

Theta range for data collection

Index ranges

Reflections collected

Independent reflections

Completeness to theta $=67.679^{\circ}$

Absorption correction

Max. and min. transmission

Refinement method

Data / restraints / parameters

Goodness-of-fit on $\mathrm{F}^{2}$

Final $\mathrm{R}$ indices $[\mathrm{I}>2 \operatorname{sigma}(\mathrm{I})=2959$ data $]$

$\mathrm{R}$ indices (all data, $0.83 \AA$ )

Absolute structure parameter

Extinction coefficient

Largest diff. peak and hole $\operatorname{cdv} 86$ (Scott Niman)

$\mathrm{C}_{19} \mathrm{H}_{25} \mathrm{~N} \mathrm{O}_{4}$

331.40

92(2) K

\section{$1.54178 \AA$}

Orthorhombic

$P 22_{12} 2_{1}$

$\mathrm{a}=7.1064(2) \AA \quad \alpha=90^{\circ}$.

$\mathrm{b}=7.9071(2) \AA \quad \beta=90^{\circ}$.

$\mathrm{c}=30.0229(7) \AA \quad \gamma=90^{\circ}$.

1687.02(7) $\AA^{3}$

4

$1.305 \mathrm{Mg} / \mathrm{m}^{3}$

$0.738 \mathrm{~mm}^{-1}$

712

colorless

$0.288 \times 0.267 \times 0.122 \mathrm{~mm}^{3}$

2.944 to $68.944^{\circ}$

$-8 \leq h \leq 8,-9 \leq k \leq 9,-36 \leq l \leq 35$

14056

$3117[\mathrm{R}(\mathrm{int})=0.0413]$

$99.9 \%$

Semi-empirical from equivalents

0.8643 and 0.7931

Full-matrix least-squares on $\mathrm{F}^{2}$

3117 / 0 / 231

1.078

$\mathrm{R} 1=0.0364, \mathrm{wR} 2=0.0918$

$\mathrm{R} 1=0.0385, \mathrm{wR} 2=0.0931$

$0.1(3)$

$0.0017(5)$

0.217 and -0.186 e. $\AA^{-3}$ 
Table 2. Atomic coordinates $\left(\times 10^{4}\right)$ and equivalent isotropic displacement parameters $\left(\AA^{2} \times 10^{3}\right)$ for $\operatorname{cdv} 86 . \mathrm{U}(\mathrm{eq})$ is defined as one third of the trace of the orthogonalized $\mathrm{U}^{\mathrm{ij}}$ tensor.

\begin{tabular}{|c|c|c|c|c|}
\hline & $\mathrm{x}$ & $\mathrm{y}$ & $\mathrm{z}$ & $\mathrm{U}(\mathrm{eq})$ \\
\hline $\mathrm{O}(1)$ & 4149(3) & 2929(2) & $3748(1)$ & $26(1)$ \\
\hline $\mathrm{O}(2)$ & $7785(3)$ & $-188(2)$ & $4782(1)$ & $28(1)$ \\
\hline $\mathrm{O}(3)$ & 7835(3) & $8402(2)$ & $2399(1)$ & $23(1)$ \\
\hline $\mathrm{O}(4)$ & $5217(3)$ & $5739(2)$ & $2416(1)$ & $25(1)$ \\
\hline $\mathrm{N}(1)$ & $4426(3)$ & 7471(3) & $3959(1)$ & $22(1)$ \\
\hline $\mathrm{C}(1)$ & 6930(4) & $5432(3)$ & $3598(1)$ & $18(1)$ \\
\hline$C(2)$ & 7163(3) & $3970(3)$ & $3938(1)$ & 19(1) \\
\hline$C(3)$ & 5764(4) & $2706(3)$ & 3989(1) & $20(1)$ \\
\hline$C(4)$ & 6005(4) & $1346(3)$ & $4273(1)$ & $22(1)$ \\
\hline$C(5)$ & $7640(4)$ & $1235(3)$ & $4527(1)$ & $22(1)$ \\
\hline$C(6)$ & $8955(4)$ & $2522(3)$ & $4512(1)$ & $24(1)$ \\
\hline$C(7)$ & $8716(4)$ & $3899(3)$ & $4220(1)$ & $20(1)$ \\
\hline $\mathrm{C}(8)$ & 10203(4) & $5260(3)$ & $4234(1)$ & $23(1)$ \\
\hline$C(9)$ & $9648(3)$ & $6842(3)$ & $3979(1)$ & $20(1)$ \\
\hline$C(10)$ & $8862(3)$ & $6316(3)$ & $3524(1)$ & $18(1)$ \\
\hline $\mathrm{C}(11)$ & $8955(3)$ & 7723(3) & $3164(1)$ & $18(1)$ \\
\hline $\mathrm{C}(12)$ & 7965(4) & $7114(3)$ & $2732(1)$ & $19(1)$ \\
\hline$C(13)$ & 6053(4) & $6353(3)$ & $2821(1)$ & $20(1)$ \\
\hline$C(14)$ & $6188(4)$ & 4863(3) & $3135(1)$ & $20(1)$ \\
\hline$C(15)$ & $5523(3)$ & $6602(3)$ & $3796(1)$ & $18(1)$ \\
\hline$C(16)$ & 2803(4) & $1600(3)$ & $3742(1)$ & $31(1)$ \\
\hline$C(17)$ & $9535(5)$ & $-455(4)$ & $5010(1)$ & $36(1)$ \\
\hline$C(18)$ & $8152(4)$ & $9420(3)$ & $3323(1)$ & $21(1)$ \\
\hline$C(19)$ & $11045(4)$ & 7971(3) & $3043(1)$ & $22(1)$ \\
\hline
\end{tabular}


Table 3. Bond lengths $[\AA]$ and angles $\left[{ }^{\circ}\right]$ for $\operatorname{cdv} 86$.

\begin{tabular}{|c|c|}
\hline $\mathrm{O}(1)-\mathrm{C}(3)$ & $1.368(3)$ \\
\hline $\mathrm{O}(1)-\mathrm{C}(16)$ & $1.421(3)$ \\
\hline $\mathrm{O}(2)-\mathrm{C}(5)$ & $1.364(3)$ \\
\hline $\mathrm{O}(2)-\mathrm{C}(17)$ & $1.436(4)$ \\
\hline $\mathrm{O}(3)-\mathrm{C}(12)$ & $1.430(3)$ \\
\hline $\mathrm{O}(4)-\mathrm{C}(13)$ & $1.440(3)$ \\
\hline $\mathrm{N}(1)-\mathrm{C}(15)$ & $1.149(3)$ \\
\hline$C(1)-C(15)$ & $1.486(3)$ \\
\hline $\mathrm{C}(1)-\mathrm{C}(2)$ & $1.551(3)$ \\
\hline$C(1)-C(14)$ & $1.553(3)$ \\
\hline$C(1)-C(10)$ & $1.556(3)$ \\
\hline $\mathrm{C}(2)-\mathrm{C}(7)$ & $1.393(3)$ \\
\hline$C(2)-C(3)$ & $1.418(4)$ \\
\hline $\mathrm{C}(3)-\mathrm{C}(4)$ & $1.384(3)$ \\
\hline$C(4)-C(5)$ & $1.392(4)$ \\
\hline $\mathrm{C}(5)-\mathrm{C}(6)$ & $1.382(4)$ \\
\hline $\mathrm{C}(6)-\mathrm{C}(7)$ & $1.408(3)$ \\
\hline $\mathrm{C}(7)-\mathrm{C}(8)$ & $1.509(4)$ \\
\hline $\mathrm{C}(8)-\mathrm{C}(9)$ & $1.518(3)$ \\
\hline$C(9)-C(10)$ & $1.532(3)$ \\
\hline$C(10)-C(11)$ & $1.554(3)$ \\
\hline$C(11)-C(18)$ & $1.535(3)$ \\
\hline$C(11)-C(19)$ & $1.541(4)$ \\
\hline$C(11)-C(12)$ & $1.550(3)$ \\
\hline$C(12)-C(13)$ & $1.510(3)$ \\
\hline$C(13)-C(14)$ & $1.510(3)$ \\
\hline $\mathrm{C}(3)-\mathrm{O}(1)-\mathrm{C}(16)$ & $118.46(19)$ \\
\hline $\mathrm{C}(5)-\mathrm{O}(2)-\mathrm{C}(17)$ & $117.0(2)$ \\
\hline$C(15)-C(1)-C(2)$ & $105.84(19)$ \\
\hline$C(15)-C(1)-C(14)$ & $108.0(2)$ \\
\hline$C(2)-C(1)-C(14)$ & $114.16(19)$ \\
\hline$C(15)-C(1)-C(10)$ & $111.74(19)$ \\
\hline$C(2)-C(1)-C(10)$ & $109.49(19)$ \\
\hline
\end{tabular}




$\begin{array}{ll}\mathrm{C}(14)-\mathrm{C}(1)-\mathrm{C}(10) & 107.63(19) \\ \mathrm{C}(7)-\mathrm{C}(2)-\mathrm{C}(3) & 117.4(2) \\ \mathrm{C}(7)-\mathrm{C}(2)-\mathrm{C}(1) & 121.0(2) \\ \mathrm{C}(3)-\mathrm{C}(2)-\mathrm{C}(1) & 121.5(2) \\ \mathrm{O}(1)-\mathrm{C}(3)-\mathrm{C}(4) & 122.0(2) \\ \mathrm{O}(1)-\mathrm{C}(3)-\mathrm{C}(2) & 116.1(2) \\ \mathrm{C}(4)-\mathrm{C}(3)-\mathrm{C}(2) & 121.9(2) \\ \mathrm{C}(3)-\mathrm{C}(4)-\mathrm{C}(5) & 119.3(2) \\ \mathrm{O}(2)-\mathrm{C}(5)-\mathrm{C}(6) & 125.1(2) \\ \mathrm{O}(2)-\mathrm{C}(5)-\mathrm{C}(4) & 114.9(2) \\ \mathrm{C}(6)-\mathrm{C}(5)-\mathrm{C}(4) & 120.0(2) \\ \mathrm{C}(5)-\mathrm{C}(6)-\mathrm{C}(7) & 120.5(2) \\ \mathrm{C}(2)-\mathrm{C}(7)-\mathrm{C}(6) & 120.4(2) \\ \mathrm{C}(2)-\mathrm{C}(7)-\mathrm{C}(8) & 122.8(2) \\ \mathrm{C}(6)-\mathrm{C}(7)-\mathrm{C}(8) & 116.8(2) \\ \mathrm{C}(7)-\mathrm{C}(8)-\mathrm{C}(9) & 113.0(2) \\ \mathrm{C}(8)-\mathrm{C}(9)-\mathrm{C}(10) & 108.70(19) \\ \mathrm{C}(9)-\mathrm{C}(10)-\mathrm{C}(11) & 114.29(19) \\ \mathrm{C}(9)-\mathrm{C}(10)-\mathrm{C}(1) & 108.50(18) \\ \mathrm{C}(11)-\mathrm{C}(10)-\mathrm{C}(1) & 117.24(19) \\ \mathrm{C}(18)-\mathrm{C}(11)-\mathrm{C}(19) & 108.7(2) \\ \mathrm{C}(18)-\mathrm{C}(11)-\mathrm{C}(12) & 111.3(2) \\ \mathrm{C}(19)-\mathrm{C}(11)-\mathrm{C}(12) & 106.32(19) \\ \mathrm{C}(18)-\mathrm{C}(11)-\mathrm{C}(10) & 113.07(19) \\ \mathrm{C}(19)-\mathrm{C}(11)-\mathrm{C}(10) & 107.22(19) \\ \mathrm{C}(12)-\mathrm{C}(11)-\mathrm{C}(10) & 109.91(18) \\ \mathrm{O}(3)-\mathrm{C}(12)-\mathrm{C}(13) & 110.46(19) \\ \mathrm{O}(3)-\mathrm{C}(12)-\mathrm{C}(11) & 113.08(19) \\ \mathrm{C}(13)-\mathrm{C}(12)-\mathrm{C}(11) & 112.63(19) \\ \mathrm{O}(4)-\mathrm{C}(13)-\mathrm{C}(12) & 110.83(19) \\ \mathrm{O}(4)-\mathrm{C}(13)-\mathrm{C}(14) & 106.90(19) \\ \mathrm{C}(12)-\mathrm{C}(13)-\mathrm{C}(14) & \\ \mathrm{C}(13)-\mathrm{C}(14)-\mathrm{C}(1) & \\ \mathrm{N}(1)-\mathrm{C}(15)-\mathrm{C}(1) & \\ & \\ & \end{array}$


Table 4. Anisotropic displacement parameters $\left(\AA^{2} \times 10^{3}\right)$ for cdv86. The anisotropic displacement factor exponent takes the form: $-2 \pi^{2}\left[h^{2} a^{* 2} U^{11}+\ldots+2 h k a^{*} b^{*} U^{12}\right]$

\begin{tabular}{lcccccc}
\hline & $\mathrm{U}^{11}$ & $\mathrm{U}^{22}$ & $\mathrm{U}^{33}$ & $\mathrm{U}^{23}$ & $\mathrm{U}^{13}$ & $\mathrm{U}^{12}$ \\
\hline $\mathrm{O}(1)$ & $17(1)$ & $24(1)$ & $36(1)$ & $4(1)$ & $-3(1)$ & $-5(1)$ \\
$\mathrm{O}(2)$ & $38(1)$ & $24(1)$ & $24(1)$ & $5(1)$ & $0(1)$ & $1(1)$ \\
$\mathrm{O}(3)$ & $23(1)$ & $26(1)$ & $21(1)$ & $3(1)$ & $-1(1)$ & $0(1)$ \\
$\mathrm{O}(4)$ & $25(1)$ & $28(1)$ & $21(1)$ & $-1(1)$ & $-6(1)$ & $-4(1)$ \\
$\mathrm{N}(1)$ & $18(1)$ & $23(1)$ & $26(1)$ & $0(1)$ & $1(1)$ & $0(1)$ \\
$\mathrm{C}(1)$ & $15(1)$ & $20(1)$ & $20(1)$ & $-2(1)$ & $-1(1)$ & $-1(1)$ \\
$\mathrm{C}(2)$ & $18(1)$ & $19(1)$ & $20(1)$ & $-2(1)$ & $2(1)$ & $1(1)$ \\
$\mathrm{C}(3)$ & $21(1)$ & $19(1)$ & $21(1)$ & $-3(1)$ & $3(1)$ & $1(1)$ \\
$\mathrm{C}(4)$ & $27(1)$ & $18(1)$ & $22(1)$ & $-3(1)$ & $6(1)$ & $-3(1)$ \\
$\mathrm{C}(5)$ & $30(2)$ & $20(1)$ & $17(1)$ & $0(1)$ & $6(1)$ & $2(1)$ \\
$\mathrm{C}(6)$ & $25(1)$ & $28(1)$ & $18(1)$ & $0(1)$ & $-1(1)$ & $2(1)$ \\
$\mathrm{C}(7)$ & $21(1)$ & $21(1)$ & $19(1)$ & $-2(1)$ & $1(1)$ & $-1(1)$ \\
$\mathrm{C}(8)$ & $20(1)$ & $27(1)$ & $23(1)$ & $2(1)$ & $-6(1)$ & $-2(1)$ \\
$\mathrm{C}(9)$ & $15(1)$ & $21(1)$ & $23(1)$ & $0(1)$ & $-1(1)$ & $-4(1)$ \\
$\mathrm{C}(10)$ & $14(1)$ & $18(1)$ & $22(1)$ & $0(1)$ & $0(1)$ & $-1(1)$ \\
$\mathrm{C}(11)$ & $16(1)$ & $19(1)$ & $20(1)$ & $0(1)$ & $0(1)$ & $-1(1)$ \\
$\mathrm{C}(12)$ & $19(1)$ & $19(1)$ & $19(1)$ & $0(1)$ & $0(1)$ & $2(1)$ \\
$\mathrm{C}(13)$ & $17(1)$ & $23(1)$ & $19(1)$ & $-3(1)$ & $-3(1)$ & $1(1)$ \\
$\mathrm{C}(14)$ & $18(1)$ & $21(1)$ & $22(1)$ & $-2(1)$ & $-1(1)$ & $-1(1)$ \\
$\mathrm{C}(15)$ & $16(1)$ & $21(1)$ & $19(1)$ & $3(1)$ & $-3(1)$ & $-5(1)$ \\
$\mathrm{C}(16)$ & $21(1)$ & $24(1)$ & $47(2)$ & $1(1)$ & $-1(1)$ & $-6(1)$ \\
$\mathrm{C}(17)$ & $50(2)$ & $31(2)$ & $27(1)$ & $7(1)$ & $-10(1)$ & $1(1)$ \\
$\mathrm{C}(18)$ & $20(1)$ & $21(1)$ & $20(1)$ & $-1(1)$ & $-1(1)$ & $0(1)$ \\
$\mathrm{C}(19)$ & $18(1)$ & $25(1)$ & $24(1)$ & $3(1)$ & $1(1)$ & $-2(1)$ \\
& & & & & & \\
\hline
\end{tabular}


Table 5. Hydrogen coordinates $\left(\times 10^{4}\right)$ and isotropic displacement parameters $\left(\AA^{2} \times 10^{3}\right)$ for cdv86.

\begin{tabular}{|c|c|c|c|c|}
\hline & $\mathrm{x}$ & $\mathrm{y}$ & $\mathrm{z}$ & $\mathrm{U}(\mathrm{eq})$ \\
\hline $\mathrm{H}(3)$ & $7110(60)$ & $9180(50)$ & $2497(11)$ & $41(10)$ \\
\hline $\mathrm{H}(4)$ & $5330(50)$ & $6520(50)$ & $2225(12)$ & $45(10)$ \\
\hline $\mathrm{H}(4 \mathrm{~A})$ & 5065 & 496 & 4295 & 27 \\
\hline $\mathrm{H}(6 \mathrm{~A})$ & 10029 & 2476 & 4700 & 28 \\
\hline $\mathrm{H}(8 \mathrm{~A})$ & 11386 & 4806 & 4107 & 28 \\
\hline $\mathrm{H}(8 \mathrm{~B})$ & 10451 & 5567 & 4548 & 28 \\
\hline $\mathrm{H}(9 \mathrm{~A})$ & 10759 & 7581 & 3938 & 24 \\
\hline $\mathrm{H}(9 \mathrm{~B})$ & 8683 & 7479 & 4148 & 24 \\
\hline $\mathrm{H}(10 \mathrm{~A})$ & 9723 & 5405 & 3416 & 22 \\
\hline $\mathrm{H}(12 \mathrm{~A})$ & 8764 & 6192 & 2605 & 23 \\
\hline $\mathrm{H}(13 \mathrm{~A})$ & 5215 & 7233 & 2955 & 24 \\
\hline $\mathrm{H}(14 \mathrm{~A})$ & 7048 & 4005 & 3008 & 24 \\
\hline $\mathrm{H}(14 \mathrm{~B})$ & 4931 & 4339 & 3169 & 24 \\
\hline $\mathrm{H}(16 \mathrm{~A})$ & 1809 & 1870 & 3527 & 46 \\
\hline $\mathrm{H}(16 \mathrm{~B})$ & 2252 & 1473 & 4039 & 46 \\
\hline $\mathrm{H}(16 \mathrm{C})$ & 3420 & 542 & 3655 & 46 \\
\hline $\mathrm{H}(17 \mathrm{~A})$ & 9506 & -1553 & 5162 & 54 \\
\hline $\mathrm{H}(17 \mathrm{~B})$ & 9722 & 445 & 5230 & 54 \\
\hline $\mathrm{H}(17 \mathrm{C})$ & 10571 & -439 & 4795 & 54 \\
\hline $\mathrm{H}(18 \mathrm{~A})$ & 8396 & 10291 & 3098 & 31 \\
\hline $\mathrm{H}(18 \mathrm{~B})$ & 8755 & 9742 & 3604 & 31 \\
\hline $\mathrm{H}(18 \mathrm{C})$ & 6792 & 9311 & 3369 & 31 \\
\hline $\mathrm{H}(19 \mathrm{~A})$ & 11149 & 8782 & 2797 & 34 \\
\hline $\mathrm{H}(19 \mathrm{~B})$ & 11588 & 6885 & 2952 & 34 \\
\hline $\mathrm{H}(19 \mathrm{C})$ & 11726 & 8404 & 3303 & 34 \\
\hline
\end{tabular}


Table 6. Torsion angles $\left[{ }^{\circ}\right]$ for $\operatorname{cdv} 86$.

\begin{tabular}{|c|c|}
\hline$C(15)-C(1)-C(2)-C(7)$ & $-102.2(3)$ \\
\hline $\mathrm{C}(14)-\mathrm{C}(1)-\mathrm{C}(2)-\mathrm{C}(7)$ & $139.1(2)$ \\
\hline$C(10)-C(1)-C(2)-C(7)$ & $18.4(3)$ \\
\hline $\mathrm{C}(15)-\mathrm{C}(1)-\mathrm{C}(2)-\mathrm{C}(3)$ & $74.5(3)$ \\
\hline$C(14)-C(1)-C(2)-C(3)$ & $-44.1(3)$ \\
\hline $\mathrm{C}(10)-\mathrm{C}(1)-\mathrm{C}(2)-\mathrm{C}(3)$ & $-164.9(2)$ \\
\hline $\mathrm{C}(16)-\mathrm{O}(1)-\mathrm{C}(3)-\mathrm{C}(4)$ & $-9.2(3)$ \\
\hline $\mathrm{C}(16)-\mathrm{O}(1)-\mathrm{C}(3)-\mathrm{C}(2)$ & $172.1(2)$ \\
\hline $\mathrm{C}(7)-\mathrm{C}(2)-\mathrm{C}(3)-\mathrm{O}(1)$ & $171.9(2)$ \\
\hline $\mathrm{C}(1)-\mathrm{C}(2)-\mathrm{C}(3)-\mathrm{O}(1)$ & $-4.9(3)$ \\
\hline$C(7)-C(2)-C(3)-C(4)$ & $-6.8(3)$ \\
\hline$C(1)-C(2)-C(3)-C(4)$ & $176.3(2)$ \\
\hline $\mathrm{O}(1)-\mathrm{C}(3)-\mathrm{C}(4)-\mathrm{C}(5)$ & $-176.7(2)$ \\
\hline$C(2)-C(3)-C(4)-C(5)$ & $2.0(3)$ \\
\hline $\mathrm{C}(17)-\mathrm{O}(2)-\mathrm{C}(5)-\mathrm{C}(6)$ & $-7.6(3)$ \\
\hline $\mathrm{C}(17)-\mathrm{O}(2)-\mathrm{C}(5)-\mathrm{C}(4)$ & $173.1(2)$ \\
\hline $\mathrm{C}(3)-\mathrm{C}(4)-\mathrm{C}(5)-\mathrm{O}(2)$ & $-177.2(2)$ \\
\hline$C(3)-C(4)-C(5)-C(6)$ & $3.5(3)$ \\
\hline $\mathrm{O}(2)-\mathrm{C}(5)-\mathrm{C}(6)-\mathrm{C}(7)$ & $176.7(2)$ \\
\hline$C(4)-C(5)-C(6)-C(7)$ & $-4.0(4)$ \\
\hline $\mathrm{C}(3)-\mathrm{C}(2)-\mathrm{C}(7)-\mathrm{C}(6)$ & $6.2(3)$ \\
\hline $\mathrm{C}(1)-\mathrm{C}(2)-\mathrm{C}(7)-\mathrm{C}(6)$ & $-176.9(2)$ \\
\hline$C(3)-C(2)-C(7)-C(8)$ & $-173.5(2)$ \\
\hline $\mathrm{C}(1)-\mathrm{C}(2)-\mathrm{C}(7)-\mathrm{C}(8)$ & $3.3(3)$ \\
\hline$C(5)-C(6)-C(7)-C(2)$ & $-1.0(4)$ \\
\hline $\mathrm{C}(5)-\mathrm{C}(6)-\mathrm{C}(7)-\mathrm{C}(8)$ & $178.8(2)$ \\
\hline$C(2)-C(7)-C(8)-C(9)$ & $11.0(3)$ \\
\hline$C(6)-C(7)-C(8)-C(9)$ & $-168.8(2)$ \\
\hline$C(7)-C(8)-C(9)-C(10)$ & $-46.6(3)$ \\
\hline $\mathrm{C}(8)-\mathrm{C}(9)-\mathrm{C}(10)-\mathrm{C}(11)$ & $-157.5(2)$ \\
\hline$C(8)-C(9)-C(10)-C(1)$ & $69.6(2)$ \\
\hline $\mathrm{C}(15)-\mathrm{C}(1)-\mathrm{C}(10)-\mathrm{C}(9)$ & $63.2(2)$ \\
\hline $\mathrm{C}(2)-\mathrm{C}(1)-\mathrm{C}(10)-\mathrm{C}(9)$ & $-53.7(2)$ \\
\hline$C(14)-C(1)-C(10)-C(9)$ & $-178.36(19)$ \\
\hline
\end{tabular}




\begin{tabular}{lc}
$\mathrm{C}(15)-\mathrm{C}(1)-\mathrm{C}(10)-\mathrm{C}(11)$ & $-68.1(3)$ \\
$\mathrm{C}(2)-\mathrm{C}(1)-\mathrm{C}(10)-\mathrm{C}(11)$ & $174.95(19)$ \\
$\mathrm{C}(14)-\mathrm{C}(1)-\mathrm{C}(10)-\mathrm{C}(11)$ & $50.3(3)$ \\
$\mathrm{C}(9)-\mathrm{C}(10)-\mathrm{C}(11)-\mathrm{C}(18)$ & $-49.8(3)$ \\
$\mathrm{C}(1)-\mathrm{C}(10)-\mathrm{C}(11)-\mathrm{C}(18)$ & $78.8(3)$ \\
$\mathrm{C}(9)-\mathrm{C}(10)-\mathrm{C}(11)-\mathrm{C}(19)$ & $70.0(2)$ \\
$\mathrm{C}(1)-\mathrm{C}(10)-\mathrm{C}(11)-\mathrm{C}(19)$ & $-161.4(2)$ \\
$\mathrm{C}(9)-\mathrm{C}(10)-\mathrm{C}(11)-\mathrm{C}(12)$ & $-174.88(19)$ \\
$\mathrm{C}(1)-\mathrm{C}(10)-\mathrm{C}(11)-\mathrm{C}(12)$ & $-46.3(3)$ \\
$\mathrm{C}(18)-\mathrm{C}(11)-\mathrm{C}(12)-\mathrm{O}(3)$ & $48.3(3)$ \\
$\mathrm{C}(19)-\mathrm{C}(11)-\mathrm{C}(12)-\mathrm{O}(3)$ & $-69.9(2)$ \\
$\mathrm{C}(10)-\mathrm{C}(11)-\mathrm{C}(12)-\mathrm{O}(3)$ & $174.35(19)$ \\
$\mathrm{C}(18)-\mathrm{C}(11)-\mathrm{C}(12)-\mathrm{C}(13)$ & $-77.8(2)$ \\
$\mathrm{C}(19)-\mathrm{C}(11)-\mathrm{C}(12)-\mathrm{C}(13)$ & $164.0(2)$ \\
$\mathrm{C}(10)-\mathrm{C}(11)-\mathrm{C}(12)-\mathrm{C}(13)$ & $48.2(3)$ \\
$\mathrm{O}(3)-\mathrm{C}(12)-\mathrm{C}(13)-\mathrm{O}(4)$ & $55.0(3)$ \\
$\mathrm{C}(11)-\mathrm{C}(12)-\mathrm{C}(13)-\mathrm{O}(4)$ & $-177.54(19)$ \\
$\mathrm{O}(3)-\mathrm{C}(12)-\mathrm{C}(13)-\mathrm{C}(14)$ & $173.81(18)$ \\
$\mathrm{C}(11)-\mathrm{C}(12)-\mathrm{C}(13)-\mathrm{C}(14)$ & $-58.7(3)$ \\
$\mathrm{O}(4)-\mathrm{C}(13)-\mathrm{C}(14)-\mathrm{C}(1)$ & $-175.50(19)$ \\
$\mathrm{C}(12)-\mathrm{C}(13)-\mathrm{C}(14)-\mathrm{C}(1)$ & $63.3(3)$ \\
$\mathrm{C}(15)-\mathrm{C}(1)-\mathrm{C}(14)-\mathrm{C}(13)$ & $-178.5(2)$ \\
$\mathrm{C}(2)-\mathrm{C}(1)-\mathrm{C}(14)-\mathrm{C}(13)$ & $-56.7(3)$ \\
$\mathrm{C}(10)-\mathrm{C}(1)-\mathrm{C}(14)-\mathrm{C}(13)$ & \\
& \\
\hline & \\
& \\
&
\end{tabular}


Table 7. Hydrogen bonds for $\operatorname{cdv} 86$ [ $\AA$ and $\left.{ }^{\circ}\right]$.

\begin{tabular}{lllll}
\hline D-H...A & d(D-H) & d(H...A & $d(D \ldots A)$ & $<($ DHA $)$ \\
\hline $\mathrm{O}(3)-\mathrm{H}(3) \ldots \mathrm{O}(4) \# 1$ & $0.85(4)$ & $2.08(4)$ & $2.904(3)$ & $162(3)$ \\
$\mathrm{O}(4)-\mathrm{H}(4) \ldots \mathrm{O}(3)$ & $0.84(4)$ & $2.38(4)$ & $2.810(3)$ & $112(3)$ \\
& & & & \\
\hline
\end{tabular}

Symmetry transformations used to generate equivalent atoms:

$\# 1-x+1, y+1 / 2,-z+1 / 2$ 
$X$-ray Data Collection, Structure Solution and Refinement for acetonide 22c (cdv85).

A colorless crystal of approximate dimensions $0.132 \times 0.218 \times 0.258 \mathrm{~mm}$ was mounted in a cryoloop and transferred to a Bruker Prospector APEX II diffractometer system. The APEX $3^{1}$ program package was used to determine the unit-cell parameters and for data collection (1-2 sec/frame scan time). The raw frame data was processed using SAINT2 and $\operatorname{SADABS}^{3}$ to yield the reflection data file. Subsequent calculations were carried out using the SHELXTL ${ }^{4}$ program package. The diffraction symmetry was $2 / m$ and the systematic absences were consistent with the monoclinic space groups $\mathrm{C2}, \mathrm{Cm}$ and $\mathrm{C} 2 / \mathrm{m}$. It was later determined that space group $C 2$ was correct.

The structure was solved by direct methods and refined on $\mathrm{F}^{2}$ by full-matrix least-squares techniques. The analytical scattering factors 5 for neutral atoms were used throughout the analysis. Hydrogen atoms were included using a riding model.

Least-squares analysis yielded wR2 $=0.0919$ and Goof $=1.047$ for 250 variables refined against 3617 data $(0.83 \AA), R 1=0.0361$ for those 3361 data with $I>2.0 \sigma(I)$. The absolute structure was assigned by refinement of the Flack parameter ${ }^{6}$ and was in agreement the synthetic method employed.

References.

1. APEX3 Version 2017.3-0, Bruker AXS, Inc.; Madison, WI 2017.

2. SAINT Version 8.38a, Bruker AXS, Inc.; Madison, WI 2013.

3. Sheldrick, G. M. SADABS, Version 2014/5, Bruker AXS, Inc.; Madison, WI 2014.

4. Sheldrick, G. M. SHELXTL, Version 2014/7, Bruker AXS, Inc.; Madison, WI 2014.

5. International Tables for Crystallography 1992, Vol. C., Dordrecht: Kluwer Academic Publishers.

6. Parsons, S., Flack, H. D., Wagner, T. Acta Cryst. B 2013, 69, 249-259. 


\section{Definitions:}

$\mathrm{wR} 2=\left[\Sigma\left[\mathrm{w}\left(\mathrm{F}_{\mathrm{o}}^{2}-\mathrm{F}_{\mathrm{c}}^{2}\right)^{2}\right] / \Sigma\left[\mathrm{w}\left(\mathrm{F}_{\mathrm{o}}^{2}\right)^{2}\right]\right]^{1 / 2}$

$\mathrm{R} 1=\Sigma|| F_{\mathrm{o}}|-| \mathrm{F}_{\mathrm{c}} \| / \Sigma\left|\mathrm{F}_{\mathrm{o}}\right|$

Goof $=S=\left[\Sigma\left[w\left(F_{o}^{2}-F_{c}^{2}\right)^{2}\right] /(n-p)\right]^{1 / 2}$ where $n$ is the number of reflections and $p$ is the total number of parameters refined.

The thermal ellipsoid plot is shown at the $50 \%$ probability level.

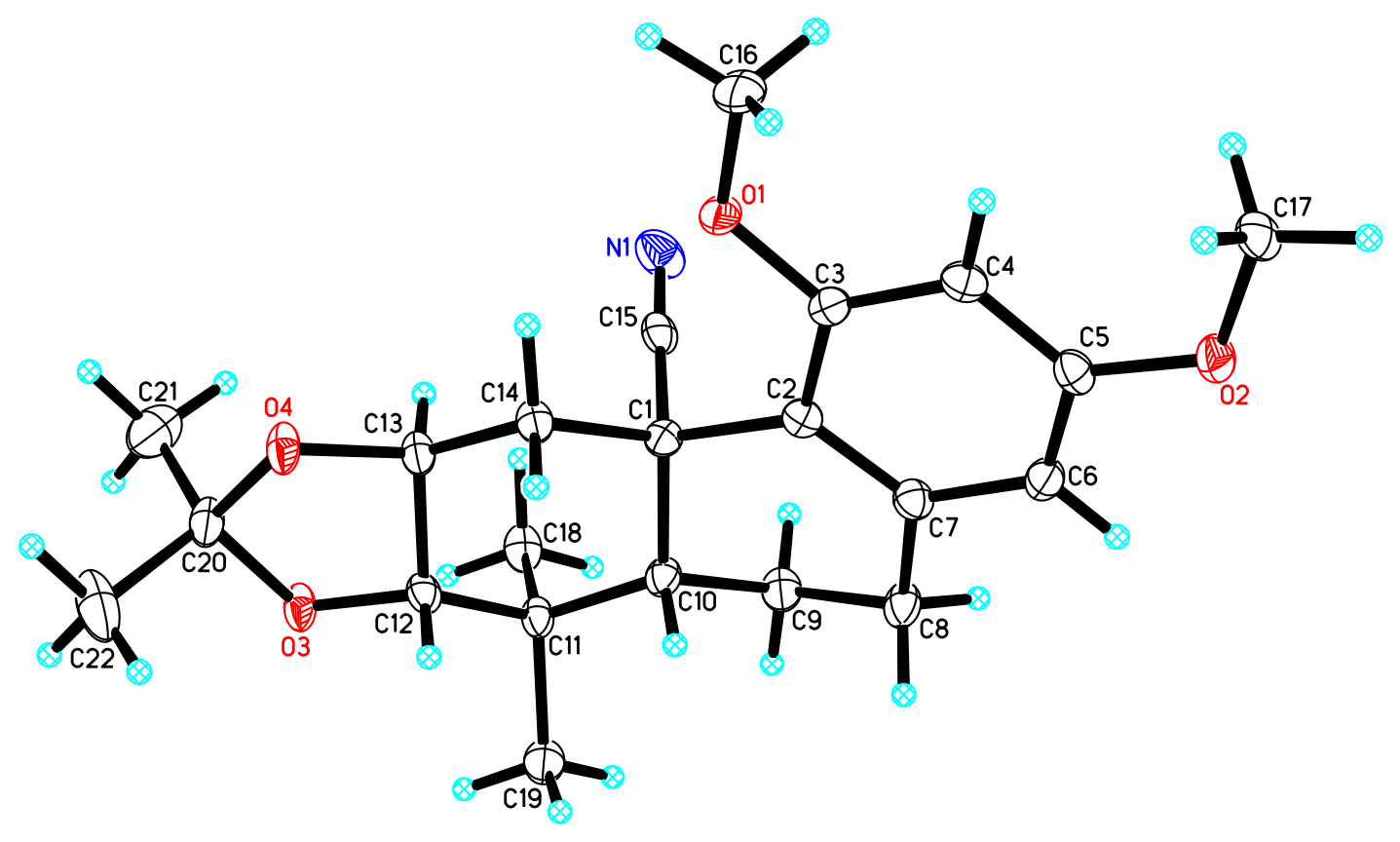


Table 1. Crystal data and structure refinement for cdv85.

Identification code

Empirical formula

Formula weight

Temperature

Wavelength

Crystal system

Space group

Unit cell dimensions

Volume

Z

Density (calculated)

Absorption coefficient

$\mathrm{F}(000)$

Crystal color

Crystal size

Theta range for data collection

Index ranges

Reflections collected

Independent reflections

Completeness to theta $=67.679^{\circ}$

Absorption correction

Max. and min. transmission

Refinement method

Data / restraints / parameters

Goodness-of-fit on $\mathrm{F}^{2}$

Final $\mathrm{R}$ indices $[\mathrm{I}>2 \operatorname{sigma}(\mathrm{I})=3361$ data $]$

$\mathrm{R}$ indices (all data, $0.83 \AA$ )

Absolute structure parameter

Largest diff. peak and hole $\operatorname{cdv} 85$ (Scott Niman)

$\mathrm{C}_{22} \mathrm{H}_{29} \mathrm{~N} \mathrm{O}_{4}$

371.46

93(2) K

\section{$1.54178 \AA$}

Monoclinic

$C 2$

$$
\begin{array}{ll}
\mathrm{a}=13.4079(5) \AA & \alpha=90^{\circ} . \\
\mathrm{b}=6.8378(3) \AA & \beta=107.187(2)^{\circ} . \\
\mathrm{c}=22.5584(9) \AA & \gamma=90^{\circ} .
\end{array}
$$

1975.82(14) $\AA^{3}$

4

$1.249 \mathrm{Mg} / \mathrm{m}^{3}$

$0.685 \mathrm{~mm}^{-1}$

800

colorless

$0.258 \times 0.218 \times 0.132 \mathrm{~mm}^{3}$

4.102 to $69.048^{\circ}$

$-16 \leq h \leq 16,-8 \leq k \leq 8,-27 \leq l \leq 27$

11051

$3617[\mathrm{R}(\mathrm{int})=0.0419]$

$99.6 \%$

Semi-empirical from equivalents

0.8643 and 0.7701

Full-matrix least-squares on $\mathrm{F}^{2}$

3617 / 1 / 250

1.047

$\mathrm{R} 1=0.0361, \mathrm{wR} 2=0.0895$

$\mathrm{R} 1=0.0395, \mathrm{wR} 2=0.0919$

0.05(16)

0.156 and -0.211 e. $\AA^{-3}$ 
Table 2. Atomic coordinates $\left(\times 10^{4}\right)$ and equivalent isotropic displacement parameters $\left(\AA^{2} \times 10^{3}\right)$ for $\operatorname{cdv} 85 . \mathrm{U}(\mathrm{eq})$ is defined as one third of the trace of the orthogonalized $\mathrm{U}^{\mathrm{ij}}$ tensor.

\begin{tabular}{|c|c|c|c|c|}
\hline & $\mathrm{x}$ & $\mathrm{y}$ & z & $\mathrm{U}(\mathrm{eq})$ \\
\hline $\mathrm{O}(1)$ & $1778(1)$ & $4679(3)$ & $6349(1)$ & $19(1)$ \\
\hline $\mathrm{O}(2)$ & $3159(1)$ & 4994(3) & $4597(1)$ & $21(1)$ \\
\hline $\mathrm{O}(3)$ & $4627(1)$ & $6618(3)$ & 9111(1) & $20(1)$ \\
\hline $\mathrm{O}(4)$ & 2893(1) & $6924(3)$ & $8535(1)$ & $21(1)$ \\
\hline $\mathrm{N}(1)$ & $3385(2)$ & $1078(3)$ & $7409(1)$ & $27(1)$ \\
\hline $\mathrm{C}(1)$ & $3802(2)$ & 4754(4) & $7233(1)$ & $15(1)$ \\
\hline$C(2)$ & $3602(2)$ & 4892(4) & $6518(1)$ & $15(1)$ \\
\hline$C(3)$ & $2587(2)$ & 4854(3) & $6097(1)$ & $15(1)$ \\
\hline$C(4)$ & $2401(2)$ & 4918(4) & $5455(1)$ & $17(1)$ \\
\hline$C(5)$ & $3244(2)$ & 4961(4) & $5214(1)$ & $17(1)$ \\
\hline$C(6)$ & $4253(2)$ & 4924(4) & $5617(1)$ & $18(1)$ \\
\hline$C(7)$ & $4438(2)$ & 4871(4) & $6259(1)$ & $17(1)$ \\
\hline $\mathrm{C}(8)$ & $5573(2)$ & 4719(4) & $6644(1)$ & $21(1)$ \\
\hline $\mathrm{C}(9)$ & $5690(2)$ & $4045(4)$ & $7300(1)$ & $20(1)$ \\
\hline $\mathrm{C}(10)$ & 4979(2) & $5284(3)$ & $7563(1)$ & $16(1)$ \\
\hline $\mathrm{C}(11)$ & $5344(2)$ & $5365(4)$ & $8289(1)$ & $17(1)$ \\
\hline $\mathrm{C}(12)$ & $4525(2)$ & $6573(4)$ & $8462(1)$ & $16(1)$ \\
\hline$C(13)$ & $3439(2)$ & 5811(4) & 8193(1) & $16(1)$ \\
\hline$C(14)$ & $3073(2)$ & $6057(3)$ & $7493(1)$ & $16(1)$ \\
\hline$C(15)$ & $3582(2)$ & $2676(4)$ & $7347(1)$ & $17(1)$ \\
\hline$C(16)$ & $736(2)$ & $4675(5)$ & $5945(1)$ & $25(1)$ \\
\hline$C(17)$ & $2129(2)$ & 4944(4) & $4168(1)$ & $19(1)$ \\
\hline $\mathrm{C}(18)$ & $5495(2)$ & $3337(4)$ & $8596(1)$ & $21(1)$ \\
\hline$C(19)$ & $6378(2)$ & $6511(4)$ & $8495(1)$ & $24(1)$ \\
\hline $\mathrm{C}(20)$ & $3591(2)$ & $6935(4)$ & $9165(1)$ & $22(1)$ \\
\hline$C(21)$ & $3337(2)$ & $5283(5)$ & $9535(1)$ & $37(1)$ \\
\hline$C(22)$ & $3544(2)$ & $8912(5)$ & $9447(1)$ & $36(1)$ \\
\hline
\end{tabular}


Table 3. Bond lengths $[\AA]$ and angles $\left[{ }^{\circ}\right]$ for $\operatorname{cdv} 85$.

\begin{tabular}{|c|c|}
\hline $\mathrm{O}(1)-\mathrm{C}(3)$ & $1.371(3)$ \\
\hline $\mathrm{O}(1)-\mathrm{C}(16)$ & $1.424(3)$ \\
\hline $\mathrm{O}(2)-\mathrm{C}(5)$ & $1.363(3)$ \\
\hline $\mathrm{O}(2)-\mathrm{C}(17)$ & $1.434(3)$ \\
\hline $\mathrm{O}(3)-\mathrm{C}(12)$ & $1.429(2)$ \\
\hline $\mathrm{O}(3)-\mathrm{C}(20)$ & $1.446(3)$ \\
\hline $\mathrm{O}(4)-\mathrm{C}(13)$ & $1.431(3)$ \\
\hline $\mathrm{O}(4)-\mathrm{C}(20)$ & $1.451(3)$ \\
\hline $\mathrm{N}(1)-\mathrm{C}(15)$ & $1.142(3)$ \\
\hline$C(1)-C(15)$ & $1.489(3)$ \\
\hline $\mathrm{C}(1)-\mathrm{C}(2)$ & $1.557(3)$ \\
\hline$C(1)-C(14)$ & $1.560(3)$ \\
\hline$C(1)-C(10)$ & $1.576(3)$ \\
\hline$C(2)-C(7)$ & $1.410(3)$ \\
\hline$C(2)-C(3)$ & $1.411(3)$ \\
\hline $\mathrm{C}(3)-\mathrm{C}(4)$ & $1.394(3)$ \\
\hline$C(4)-C(5)$ & $1.392(3)$ \\
\hline $\mathrm{C}(4)-\mathrm{H}(4 \mathrm{~A})$ & 0.9500 \\
\hline$C(5)-C(6)$ & $1.390(3)$ \\
\hline$C(6)-C(7)$ & $1.394(3)$ \\
\hline $\mathrm{C}(6)-\mathrm{H}(6 \mathrm{~A})$ & 0.9500 \\
\hline$C(7)-C(8)$ & $1.517(3)$ \\
\hline $\mathrm{C}(8)-\mathrm{C}(9)$ & $1.514(3)$ \\
\hline $\mathrm{C}(8)-\mathrm{H}(8 \mathrm{~A})$ & 0.9900 \\
\hline $\mathrm{C}(8)-\mathrm{H}(8 \mathrm{~B})$ & 0.9900 \\
\hline$C(9)-C(10)$ & $1.521(3)$ \\
\hline $\mathrm{C}(9)-\mathrm{H}(9 \mathrm{~A})$ & 0.9900 \\
\hline $\mathrm{C}(9)-\mathrm{H}(9 \mathrm{~B})$ & 0.9900 \\
\hline$C(10)-C(11)$ & $1.566(3)$ \\
\hline $\mathrm{C}(10)-\mathrm{H}(10 \mathrm{~A})$ & 1.0000 \\
\hline$C(11)-C(12)$ & $1.515(3)$ \\
\hline$C(11)-C(18)$ & $1.537(3)$ \\
\hline$C(11)-C(19)$ & $1.540(3)$ \\
\hline$C(12)-C(13)$ & $1.495(3)$ \\
\hline
\end{tabular}




\begin{tabular}{|c|c|}
\hline $\mathrm{C}(12)-\mathrm{H}(12 \mathrm{~A})$ & 1.0000 \\
\hline$C(13)-C(14)$ & $1.517(3)$ \\
\hline$C(13)-H(13 A)$ & 1.0000 \\
\hline $\mathrm{C}(14)-\mathrm{H}(14 \mathrm{~A})$ & 0.9900 \\
\hline $\mathrm{C}(14)-\mathrm{H}(14 \mathrm{~B})$ & 0.9900 \\
\hline $\mathrm{C}(16)-\mathrm{H}(16 \mathrm{~A})$ & 0.9800 \\
\hline $\mathrm{C}(16)-\mathrm{H}(16 \mathrm{~B})$ & 0.9800 \\
\hline $\mathrm{C}(16)-\mathrm{H}(16 \mathrm{C})$ & 0.9800 \\
\hline $\mathrm{C}(17)-\mathrm{H}(17 \mathrm{~A})$ & 0.9800 \\
\hline $\mathrm{C}(17)-\mathrm{H}(17 \mathrm{~B})$ & 0.9800 \\
\hline $\mathrm{C}(17)-\mathrm{H}(17 \mathrm{C})$ & 0.9800 \\
\hline $\mathrm{C}(18)-\mathrm{H}(18 \mathrm{~A})$ & 0.9800 \\
\hline $\mathrm{C}(18)-\mathrm{H}(18 \mathrm{~B})$ & 0.9800 \\
\hline $\mathrm{C}(18)-\mathrm{H}(18 \mathrm{C})$ & 0.9800 \\
\hline $\mathrm{C}(19)-\mathrm{H}(19 \mathrm{~A})$ & 0.9800 \\
\hline $\mathrm{C}(19)-\mathrm{H}(19 \mathrm{~B})$ & 0.9800 \\
\hline C(19)-H(19C) & 0.9800 \\
\hline$C(20)-C(21)$ & $1.502(4)$ \\
\hline$C(20)-C(22)$ & $1.503(4)$ \\
\hline $\mathrm{C}(21)-\mathrm{H}(21 \mathrm{C})$ & 0.9800 \\
\hline $\mathrm{C}(21)-\mathrm{H}(21 \mathrm{D})$ & 0.9800 \\
\hline $\mathrm{C}(21)-\mathrm{H}(21 \mathrm{~A})$ & 0.9800 \\
\hline $\mathrm{C}(22)-\mathrm{H}(22 \mathrm{D})$ & 0.9800 \\
\hline $\mathrm{C}(22)-\mathrm{H}(22 \mathrm{~A})$ & 0.9800 \\
\hline $\mathrm{C}(22)-\mathrm{H}(22 \mathrm{~B})$ & 0.9800 \\
\hline $\mathrm{C}(3)-\mathrm{O}(1)-\mathrm{C}(16)$ & $118.78(17)$ \\
\hline $\mathrm{C}(5)-\mathrm{O}(2)-\mathrm{C}(17)$ & $117.62(18)$ \\
\hline $\mathrm{C}(12)-\mathrm{O}(3)-\mathrm{C}(20)$ & $106.55(15)$ \\
\hline $\mathrm{C}(13)-\mathrm{O}(4)-\mathrm{C}(20)$ & $104.28(16)$ \\
\hline$C(15)-C(1)-C(2)$ & $104.77(19)$ \\
\hline$C(15)-C(1)-C(14)$ & $107.58(19)$ \\
\hline $\mathrm{C}(2)-\mathrm{C}(1)-\mathrm{C}(14)$ & $114.81(18)$ \\
\hline$C(15)-C(1)-C(10)$ & $111.08(19)$ \\
\hline $\mathrm{C}(2)-\mathrm{C}(1)-\mathrm{C}(10)$ & $108.47(17)$ \\
\hline$C(14)-C(1)-C(10)$ & $110.04(18)$ \\
\hline
\end{tabular}




\begin{tabular}{|c|c|}
\hline$C(7)-C(2)-C(3)$ & $116.5(2)$ \\
\hline$C(7)-C(2)-C(1)$ & $121.02(19)$ \\
\hline$C(3)-C(2)-C(1)$ & $122.23(19)$ \\
\hline $\mathrm{O}(1)-\mathrm{C}(3)-\mathrm{C}(4)$ & $120.77(18)$ \\
\hline $\mathrm{O}(1)-\mathrm{C}(3)-\mathrm{C}(2)$ & $116.46(19)$ \\
\hline$C(4)-C(3)-C(2)$ & $122.7(2)$ \\
\hline$C(5)-C(4)-C(3)$ & $119.23(19)$ \\
\hline $\mathrm{C}(5)-\mathrm{C}(4)-\mathrm{H}(4 \mathrm{~A})$ & 120.4 \\
\hline $\mathrm{C}(3)-\mathrm{C}(4)-\mathrm{H}(4 \mathrm{~A})$ & 120.4 \\
\hline $\mathrm{O}(2)-\mathrm{C}(5)-\mathrm{C}(6)$ & $116.1(2)$ \\
\hline $\mathrm{O}(2)-\mathrm{C}(5)-\mathrm{C}(4)$ & $124.5(2)$ \\
\hline$C(6)-C(5)-C(4)$ & $119.4(2)$ \\
\hline$C(5)-C(6)-C(7)$ & $121.2(2)$ \\
\hline $\mathrm{C}(5)-\mathrm{C}(6)-\mathrm{H}(6 \mathrm{~A})$ & 119.4 \\
\hline$C(7)-C(6)-H(6 A)$ & 119.4 \\
\hline$C(6)-C(7)-C(2)$ & $120.8(2)$ \\
\hline$C(6)-C(7)-C(8)$ & $115.9(2)$ \\
\hline $\mathrm{C}(2)-\mathrm{C}(7)-\mathrm{C}(8)$ & $123.3(2)$ \\
\hline$C(9)-C(8)-C(7)$ & $112.17(19)$ \\
\hline $\mathrm{C}(9)-\mathrm{C}(8)-\mathrm{H}(8 \mathrm{~A})$ & 109.2 \\
\hline $\mathrm{C}(7)-\mathrm{C}(8)-\mathrm{H}(8 \mathrm{~A})$ & 109.2 \\
\hline $\mathrm{C}(9)-\mathrm{C}(8)-\mathrm{H}(8 \mathrm{~B})$ & 109.2 \\
\hline $\mathrm{C}(7)-\mathrm{C}(8)-\mathrm{H}(8 \mathrm{~B})$ & 109.2 \\
\hline $\mathrm{H}(8 \mathrm{~A})-\mathrm{C}(8)-\mathrm{H}(8 \mathrm{~B})$ & 107.9 \\
\hline$C(8)-C(9)-C(10)$ & 108.46(19) \\
\hline $\mathrm{C}(8)-\mathrm{C}(9)-\mathrm{H}(9 \mathrm{~A})$ & 110.0 \\
\hline $\mathrm{C}(10)-\mathrm{C}(9)-\mathrm{H}(9 \mathrm{~A})$ & 110.0 \\
\hline $\mathrm{C}(8)-\mathrm{C}(9)-\mathrm{H}(9 \mathrm{~B})$ & 110.0 \\
\hline $\mathrm{C}(10)-\mathrm{C}(9)-\mathrm{H}(9 \mathrm{~B})$ & 110.0 \\
\hline $\mathrm{H}(9 \mathrm{~A})-\mathrm{C}(9)-\mathrm{H}(9 \mathrm{~B})$ & 108.4 \\
\hline$C(9)-C(10)-C(11)$ & $112.95(19)$ \\
\hline $\mathrm{C}(9)-\mathrm{C}(10)-\mathrm{C}(1)$ & $110.10(19)$ \\
\hline $\mathrm{C}(11)-\mathrm{C}(10)-\mathrm{C}(1)$ & $117.52(18)$ \\
\hline $\mathrm{C}(9)-\mathrm{C}(10)-\mathrm{H}(10 \mathrm{~A})$ & 105.0 \\
\hline $\mathrm{C}(11)-\mathrm{C}(10)-\mathrm{H}(10 \mathrm{~A})$ & 105.0 \\
\hline $\mathrm{C}(1)-\mathrm{C}(10)-\mathrm{H}(10 \mathrm{~A})$ & 105.0 \\
\hline
\end{tabular}




\begin{tabular}{|c|c|}
\hline $\mathrm{C}(12)-\mathrm{C}(11)-\mathrm{C}(18)$ & $112.6(2)$ \\
\hline $\mathrm{C}(12)-\mathrm{C}(11)-\mathrm{C}(19)$ & $107.6(2)$ \\
\hline $\mathrm{C}(18)-\mathrm{C}(11)-\mathrm{C}(19)$ & $109.67(19)$ \\
\hline$C(12)-C(11)-C(10)$ & $105.23(17)$ \\
\hline $\mathrm{C}(18)-\mathrm{C}(11)-\mathrm{C}(10)$ & $113.44(19)$ \\
\hline $\mathrm{C}(19)-\mathrm{C}(11)-\mathrm{C}(10)$ & $107.98(19)$ \\
\hline $\mathrm{O}(3)-\mathrm{C}(12)-\mathrm{C}(13)$ & $102.21(17)$ \\
\hline $\mathrm{O}(3)-\mathrm{C}(12)-\mathrm{C}(11)$ & $114.72(17)$ \\
\hline $\mathrm{C}(13)-\mathrm{C}(12)-\mathrm{C}(11)$ & $113.44(19)$ \\
\hline $\mathrm{O}(3)-\mathrm{C}(12)-\mathrm{H}(12 \mathrm{~A})$ & 108.7 \\
\hline $\mathrm{C}(13)-\mathrm{C}(12)-\mathrm{H}(12 \mathrm{~A})$ & 108.7 \\
\hline $\mathrm{C}(11)-\mathrm{C}(12)-\mathrm{H}(12 \mathrm{~A})$ & 108.7 \\
\hline $\mathrm{O}(4)-\mathrm{C}(13)-\mathrm{C}(12)$ & $100.75(17)$ \\
\hline $\mathrm{O}(4)-\mathrm{C}(13)-\mathrm{C}(14)$ & $116.25(18)$ \\
\hline$C(12)-C(13)-C(14)$ & $111.20(19)$ \\
\hline $\mathrm{O}(4)-\mathrm{C}(13)-\mathrm{H}(13 \mathrm{~A})$ & 109.4 \\
\hline $\mathrm{C}(12)-\mathrm{C}(13)-\mathrm{H}(13 \mathrm{~A})$ & 109.4 \\
\hline $\mathrm{C}(14)-\mathrm{C}(13)-\mathrm{H}(13 \mathrm{~A})$ & 109.4 \\
\hline$C(13)-C(14)-C(1)$ & $106.33(17)$ \\
\hline $\mathrm{C}(13)-\mathrm{C}(14)-\mathrm{H}(14 \mathrm{~A})$ & 110.5 \\
\hline $\mathrm{C}(1)-\mathrm{C}(14)-\mathrm{H}(14 \mathrm{~A})$ & 110.5 \\
\hline $\mathrm{C}(13)-\mathrm{C}(14)-\mathrm{H}(14 \mathrm{~B})$ & 110.5 \\
\hline $\mathrm{C}(1)-\mathrm{C}(14)-\mathrm{H}(14 \mathrm{~B})$ & 110.5 \\
\hline $\mathrm{H}(14 \mathrm{~A})-\mathrm{C}(14)-\mathrm{H}(14 \mathrm{~B})$ & 108.7 \\
\hline $\mathrm{N}(1)-\mathrm{C}(15)-\mathrm{C}(1)$ & $177.0(3)$ \\
\hline $\mathrm{O}(1)-\mathrm{C}(16)-\mathrm{H}(16 \mathrm{~A})$ & 109.5 \\
\hline $\mathrm{O}(1)-\mathrm{C}(16)-\mathrm{H}(16 \mathrm{~B})$ & 109.5 \\
\hline $\mathrm{H}(16 \mathrm{~A})-\mathrm{C}(16)-\mathrm{H}(16 \mathrm{~B})$ & 109.5 \\
\hline $\mathrm{O}(1)-\mathrm{C}(16)-\mathrm{H}(16 \mathrm{C})$ & 109.5 \\
\hline $\mathrm{H}(16 \mathrm{~A})-\mathrm{C}(16)-\mathrm{H}(16 \mathrm{C})$ & 109.5 \\
\hline $\mathrm{H}(16 \mathrm{~B})-\mathrm{C}(16)-\mathrm{H}(16 \mathrm{C})$ & 109.5 \\
\hline $\mathrm{O}(2)-\mathrm{C}(17)-\mathrm{H}(17 \mathrm{~A})$ & 109.5 \\
\hline $\mathrm{O}(2)-\mathrm{C}(17)-\mathrm{H}(17 \mathrm{~B})$ & 109.5 \\
\hline $\mathrm{H}(17 \mathrm{~A})-\mathrm{C}(17)-\mathrm{H}(17 \mathrm{~B})$ & 109.5 \\
\hline $\mathrm{O}(2)-\mathrm{C}(17)-\mathrm{H}(17 \mathrm{C})$ & 109.5 \\
\hline $\mathrm{H}(17 \mathrm{~A})-\mathrm{C}(17)-\mathrm{H}(17 \mathrm{C})$ & 109.5 \\
\hline
\end{tabular}




$\begin{array}{ll}\mathrm{H}(17 \mathrm{~B})-\mathrm{C}(17)-\mathrm{H}(17 \mathrm{C}) & 109.5 \\ \mathrm{C}(11)-\mathrm{C}(18)-\mathrm{H}(18 \mathrm{~A}) & 109.5 \\ \mathrm{C}(11)-\mathrm{C}(18)-\mathrm{H}(18 \mathrm{~B}) & 109.5 \\ \mathrm{H}(18 \mathrm{~A})-\mathrm{C}(18)-\mathrm{H}(18 \mathrm{~B}) & 109.5 \\ \mathrm{C}(11)-\mathrm{C}(18)-\mathrm{H}(18 \mathrm{C}) & 109.5 \\ \mathrm{H}(18 \mathrm{~A})-\mathrm{C}(18)-\mathrm{H}(18 \mathrm{C}) & 109.5 \\ \mathrm{H}(18 \mathrm{~B})-\mathrm{C}(18)-\mathrm{H}(18 \mathrm{C}) & 109.5 \\ \mathrm{C}(11)-\mathrm{C}(19)-\mathrm{H}(19 A) & 109.5 \\ \mathrm{C}(11)-\mathrm{C}(19)-\mathrm{H}(19 B) & 109.5 \\ \mathrm{H}(19 \mathrm{~A})-\mathrm{C}(19)-\mathrm{H}(19 \mathrm{~B}) & 109.5 \\ \mathrm{C}(11)-\mathrm{C}(19)-\mathrm{H}(19 \mathrm{C}) & 109.5 \\ \mathrm{H}(19 \mathrm{~A})-\mathrm{C}(19)-\mathrm{H}(19 \mathrm{C}) & 109.5 \\ \mathrm{H}(19 \mathrm{~B})-\mathrm{C}(19)-\mathrm{H}(19 \mathrm{C}) & 109.5 \\ \mathrm{O}(3)-\mathrm{C}(20)-\mathrm{O}(4) & 105.94(17) \\ \mathrm{O}(3)-\mathrm{C}(20)-\mathrm{C}(21) & 108.4(2) \\ \mathrm{O}(4)-\mathrm{C}(20)-\mathrm{C}(21) & 110.7(2) \\ \mathrm{O}(3)-\mathrm{C}(20)-\mathrm{C}(22) & 109.3(2) \\ \mathrm{O}(4)-\mathrm{C}(20)-\mathrm{C}(22) & 108.9(2) \\ \mathrm{C}(21)-\mathrm{C}(20)-\mathrm{C}(22) & 113.3(2) \\ \mathrm{C}(20)-\mathrm{C}(21)-\mathrm{H}(21 \mathrm{C}) & 109.5 \\ \mathrm{C}(20)-\mathrm{C}(21)-\mathrm{H}(21 \mathrm{D}) & 109.5 \\ \mathrm{H}(21 \mathrm{C})-\mathrm{C}(21)-\mathrm{H}(21 \mathrm{D}) & 109.5 \\ \mathrm{C}(20)-\mathrm{C}(21)-\mathrm{H}(21 \mathrm{~A}) & 109.5 \\ \mathrm{H}(21 \mathrm{C})-\mathrm{C}(21)-\mathrm{H}(21 \mathrm{~A}) & 109.5 \\ \mathrm{H}(21 \mathrm{D})-\mathrm{C}(21)-\mathrm{H}(21 \mathrm{~A}) & 109.5 \\ \mathrm{C}(20)-\mathrm{C}(22)-\mathrm{H}(22 \mathrm{D}) & 109.5 \\ \mathrm{C}(20)-\mathrm{C}(22)-\mathrm{H}(22 \mathrm{~A}) & 109.5 \\ \mathrm{H}(22 \mathrm{D})-\mathrm{C}(22)-\mathrm{H}(22 \mathrm{~A}) & 109.5 \\ \mathrm{C}(20)-\mathrm{C}(22)-\mathrm{H}(22 \mathrm{~B}) & 109.5 \\ \mathrm{H}(22 \mathrm{D})-\mathrm{C}(22)-\mathrm{H}(22 \mathrm{~B}) & 109.5 \\ \mathrm{H}(22 \mathrm{~A})-\mathrm{C}(22)-\mathrm{H}(22 \mathrm{~B}) & 109.5 \\ & \end{array}$


Table 4. Anisotropic displacement parameters $\left(\AA^{2} \times 10^{3}\right)$ for cdv85. The anisotropic displacement factor exponent takes the form: $-2 \pi^{2}\left[h^{2} a^{* 2} U^{11}+\ldots+2 h k a^{*} b^{*} U^{12}\right]$

\begin{tabular}{|c|c|c|c|c|c|c|}
\hline & $\mathrm{U}^{11}$ & $\mathrm{U}^{22}$ & $\mathrm{U}^{33}$ & $\mathrm{U}^{23}$ & $\mathrm{U}^{13}$ & $\mathrm{U}^{12}$ \\
\hline $\mathrm{O}(1)$ & $11(1)$ & $32(1)$ & $14(1)$ & $-1(1)$ & $2(1)$ & $-3(1)$ \\
\hline $\mathrm{O}(2)$ & $20(1)$ & $29(1)$ & $12(1)$ & $0(1)$ & $4(1)$ & $-2(1)$ \\
\hline $\mathrm{O}(3)$ & $12(1)$ & $33(1)$ & $13(1)$ & $-5(1)$ & $2(1)$ & $5(1)$ \\
\hline $\mathrm{O}(4)$ & $12(1)$ & $36(1)$ & $12(1)$ & $-5(1)$ & $2(1)$ & $7(1)$ \\
\hline $\mathrm{N}(1)$ & $39(1)$ & $20(1)$ & $21(1)$ & $0(1)$ & $5(1)$ & $-5(1)$ \\
\hline $\mathrm{C}(1)$ & $13(1)$ & $16(1)$ & $14(1)$ & $1(1)$ & $4(1)$ & $1(1)$ \\
\hline$C(2)$ & $16(1)$ & $13(1)$ & $16(1)$ & $1(1)$ & $4(1)$ & $1(1)$ \\
\hline$C(3)$ & $14(1)$ & $13(1)$ & $19(1)$ & $0(1)$ & $6(1)$ & $1(1)$ \\
\hline $\mathrm{C}(4)$ & $16(1)$ & $17(1)$ & $17(1)$ & $0(1)$ & $2(1)$ & $0(1)$ \\
\hline$C(5)$ & $21(1)$ & $16(1)$ & $13(1)$ & $0(1)$ & $3(1)$ & $0(1)$ \\
\hline$C(6)$ & $17(1)$ & $21(1)$ & $17(1)$ & $0(1)$ & $7(1)$ & $-1(1)$ \\
\hline$C(7)$ & $16(1)$ & $18(1)$ & $15(1)$ & $0(1)$ & $3(1)$ & $1(1)$ \\
\hline $\mathrm{C}(8)$ & $14(1)$ & $32(1)$ & $18(1)$ & $-2(1)$ & $6(1)$ & $2(1)$ \\
\hline $\mathrm{C}(9)$ & $14(1)$ & $27(1)$ & $17(1)$ & $-1(1)$ & $3(1)$ & $4(1)$ \\
\hline$C(10)$ & $13(1)$ & $22(1)$ & $15(1)$ & $0(1)$ & $4(1)$ & $1(1)$ \\
\hline $\mathrm{C}(11)$ & $13(1)$ & $25(1)$ & $12(1)$ & $-3(1)$ & $3(1)$ & $2(1)$ \\
\hline$C(12)$ & $15(1)$ & $20(1)$ & $11(1)$ & $-2(1)$ & $2(1)$ & $1(1)$ \\
\hline$C(13)$ & $14(1)$ & $22(1)$ & $12(1)$ & $-2(1)$ & $3(1)$ & $4(1)$ \\
\hline$C(14)$ & $13(1)$ & $20(1)$ & $13(1)$ & $-2(1)$ & $2(1)$ & $4(1)$ \\
\hline$C(15)$ & $18(1)$ & $21(1)$ & $11(1)$ & $-1(1)$ & $2(1)$ & $1(1)$ \\
\hline$C(16)$ & $12(1)$ & $41(2)$ & $21(1)$ & $-1(1)$ & $2(1)$ & $-3(1)$ \\
\hline$C(17)$ & $20(1)$ & $22(1)$ & $13(1)$ & $2(1)$ & $1(1)$ & $0(1)$ \\
\hline$C(18)$ & $18(1)$ & $27(1)$ & $15(1)$ & $1(1)$ & $3(1)$ & $7(1)$ \\
\hline C(19) & $14(1)$ & $38(2)$ & $18(1)$ & $-5(1)$ & $3(1)$ & $-2(1)$ \\
\hline$C(20)$ & $12(1)$ & $40(2)$ & $13(1)$ & $-5(1)$ & $2(1)$ & $4(1)$ \\
\hline$C(21)$ & $24(1)$ & $66(2)$ & $17(1)$ & $4(1)$ & $2(1)$ & $-9(1)$ \\
\hline$C(22)$ & $26(1)$ & $53(2)$ & $25(1)$ & $-16(1)$ & $-1(1)$ & $16(1)$ \\
\hline
\end{tabular}


Table 5. Hydrogen coordinates $\left(\times 10^{4}\right)$ and isotropic displacement parameters $\left(\AA^{2} \times 10^{3}\right)$ for cdv85.

\begin{tabular}{|c|c|c|c|c|}
\hline & $\mathrm{x}$ & $\mathrm{y}$ & $\mathrm{z}$ & $\mathrm{U}(\mathrm{eq})$ \\
\hline $\mathrm{H}(4 \mathrm{~A})$ & 1706 & 4933 & 5186 & 21 \\
\hline $\mathrm{H}(6 \mathrm{~A})$ & 4829 & 4935 & 5452 & 22 \\
\hline $\mathrm{H}(8 \mathrm{~A})$ & 5936 & 3785 & 6443 & 25 \\
\hline $\mathrm{H}(8 \mathrm{~B})$ & 5911 & 6012 & 6657 & 25 \\
\hline $\mathrm{H}(9 \mathrm{~A})$ & 6424 & 4194 & 7558 & 24 \\
\hline $\mathrm{H}(9 \mathrm{~B})$ & 5496 & 2648 & 7300 & 24 \\
\hline $\mathrm{H}(10 \mathrm{~A})$ & 5064 & 6650 & 7427 & 20 \\
\hline $\mathrm{H}(12 \mathrm{~A})$ & 4554 & 7944 & 8314 & 19 \\
\hline $\mathrm{H}(13 \mathrm{~A})$ & 3419 & 4394 & 8298 & 20 \\
\hline $\mathrm{H}(14 \mathrm{~A})$ & 3125 & 7444 & 7380 & 19 \\
\hline $\mathrm{H}(14 \mathrm{~B})$ & 2337 & 5632 & 7323 & 19 \\
\hline $\mathrm{H}(16 \mathrm{~A})$ & 245 & 4600 & 6191 & 38 \\
\hline $\mathrm{H}(16 \mathrm{~B})$ & 636 & 3544 & 5667 & 38 \\
\hline $\mathrm{H}(16 \mathrm{C})$ & 610 & 5880 & 5699 & 38 \\
\hline $\mathrm{H}(17 \mathrm{~A})$ & 2176 & 4989 & 3742 & 29 \\
\hline $\mathrm{H}(17 \mathrm{~B})$ & 1730 & 6073 & 4240 & 29 \\
\hline $\mathrm{H}(17 \mathrm{C})$ & 1777 & 3735 & 4227 & 29 \\
\hline $\mathrm{H}(18 \mathrm{~A})$ & 5708 & 3487 & 9048 & 31 \\
\hline $\mathrm{H}(18 \mathrm{~B})$ & 6035 & 2621 & 8473 & 31 \\
\hline $\mathrm{H}(18 \mathrm{C})$ & 4836 & 2609 & 8462 & 31 \\
\hline $\mathrm{H}(19 \mathrm{~A})$ & 6574 & 6737 & 8944 & 36 \\
\hline $\mathrm{H}(19 \mathrm{~B})$ & 6292 & 7770 & 8278 & 36 \\
\hline $\mathrm{H}(19 \mathrm{C})$ & 6928 & 5755 & 8394 & 36 \\
\hline $\mathrm{H}(21 \mathrm{C})$ & 3358 & 4043 & 9322 & 55 \\
\hline $\mathrm{H}(21 \mathrm{D})$ & 2637 & 5478 & 9578 & 55 \\
\hline $\mathrm{H}(21 \mathrm{~A})$ & 3850 & 5252 & 9947 & 55 \\
\hline $\mathrm{H}(22 \mathrm{D})$ & 3749 & 9918 & 9196 & 54 \\
\hline $\mathrm{H}(22 \mathrm{~A})$ & 4022 & 8941 & 9870 & 54 \\
\hline $\mathrm{H}(22 \mathrm{~B})$ & 2831 & 9165 & 9458 & 54 \\
\hline
\end{tabular}


Table 6. Torsion angles $\left[{ }^{\circ}\right]$ for $\operatorname{cdv} 85$.

\begin{tabular}{|c|c|}
\hline$C(15)-C(1)-C(2)-C(7)$ & $-100.6(3)$ \\
\hline $\mathrm{C}(14)-\mathrm{C}(1)-\mathrm{C}(2)-\mathrm{C}(7)$ & $141.6(2)$ \\
\hline$C(10)-C(1)-C(2)-C(7)$ & $18.0(3)$ \\
\hline $\mathrm{C}(15)-\mathrm{C}(1)-\mathrm{C}(2)-\mathrm{C}(3)$ & $73.1(3)$ \\
\hline $\mathrm{C}(14)-\mathrm{C}(1)-\mathrm{C}(2)-\mathrm{C}(3)$ & $-44.7(3)$ \\
\hline $\mathrm{C}(10)-\mathrm{C}(1)-\mathrm{C}(2)-\mathrm{C}(3)$ & $-168.2(2)$ \\
\hline $\mathrm{C}(16)-\mathrm{O}(1)-\mathrm{C}(3)-\mathrm{C}(4)$ & $-3.5(3)$ \\
\hline $\mathrm{C}(16)-\mathrm{O}(1)-\mathrm{C}(3)-\mathrm{C}(2)$ & $178.7(2)$ \\
\hline $\mathrm{C}(7)-\mathrm{C}(2)-\mathrm{C}(3)-\mathrm{O}(1)$ & $173.7(2)$ \\
\hline $\mathrm{C}(1)-\mathrm{C}(2)-\mathrm{C}(3)-\mathrm{O}(1)$ & $-0.3(3)$ \\
\hline$C(7)-C(2)-C(3)-C(4)$ & $-4.0(3)$ \\
\hline $\mathrm{C}(1)-\mathrm{C}(2)-\mathrm{C}(3)-\mathrm{C}(4)$ & $-178.0(2)$ \\
\hline $\mathrm{O}(1)-\mathrm{C}(3)-\mathrm{C}(4)-\mathrm{C}(5)$ & $-175.6(2)$ \\
\hline$C(2)-C(3)-C(4)-C(5)$ & $2.1(4)$ \\
\hline $\mathrm{C}(17)-\mathrm{O}(2)-\mathrm{C}(5)-\mathrm{C}(6)$ & $177.3(2)$ \\
\hline $\mathrm{C}(17)-\mathrm{O}(2)-\mathrm{C}(5)-\mathrm{C}(4)$ & $-1.2(4)$ \\
\hline $\mathrm{C}(3)-\mathrm{C}(4)-\mathrm{C}(5)-\mathrm{O}(2)$ & $178.9(2)$ \\
\hline$C(3)-C(4)-C(5)-C(6)$ & $0.5(4)$ \\
\hline $\mathrm{O}(2)-\mathrm{C}(5)-\mathrm{C}(6)-\mathrm{C}(7)$ & $-179.4(2)$ \\
\hline$C(4)-C(5)-C(6)-C(7)$ & $-0.9(4)$ \\
\hline$C(5)-C(6)-C(7)-C(2)$ & $-1.3(4)$ \\
\hline$C(5)-C(6)-C(7)-C(8)$ & $176.7(2)$ \\
\hline$C(3)-C(2)-C(7)-C(6)$ & $3.6(4)$ \\
\hline$C(1)-C(2)-C(7)-C(6)$ & $177.7(2)$ \\
\hline$C(3)-C(2)-C(7)-C(8)$ & $-174.2(2)$ \\
\hline $\mathrm{C}(1)-\mathrm{C}(2)-\mathrm{C}(7)-\mathrm{C}(8)$ & $-0.1(4)$ \\
\hline $\mathrm{C}(6)-\mathrm{C}(7)-\mathrm{C}(8)-\mathrm{C}(9)$ & $-162.2(2)$ \\
\hline $\mathrm{C}(2)-\mathrm{C}(7)-\mathrm{C}(8)-\mathrm{C}(9)$ & $15.7(4)$ \\
\hline$C(7)-C(8)-C(9)-C(10)$ & $-49.3(3)$ \\
\hline $\mathrm{C}(8)-\mathrm{C}(9)-\mathrm{C}(10)-\mathrm{C}(11)$ & $-156.2(2)$ \\
\hline$C(8)-C(9)-C(10)-C(1)$ & $70.3(2)$ \\
\hline $\mathrm{C}(15)-\mathrm{C}(1)-\mathrm{C}(10)-\mathrm{C}(9)$ & $62.2(2)$ \\
\hline$C(2)-C(1)-C(10)-C(9)$ & $-52.4(2)$ \\
\hline $\mathrm{C}(14)-\mathrm{C}(1)-\mathrm{C}(10)-\mathrm{C}(9)$ & $-178.75(19)$ \\
\hline
\end{tabular}




\begin{tabular}{|c|c|}
\hline$C(15)-C(1)-C(10)-C(11)$ & $-69.0(3)$ \\
\hline$C(2)-C(1)-C(10)-C(11)$ & $176.4(2)$ \\
\hline$C(14)-C(1)-C(10)-C(11)$ & $50.0(3)$ \\
\hline$C(9)-C(10)-C(11)-C(12)$ & $-177.6(2)$ \\
\hline$C(1)-C(10)-C(11)-C(12)$ & $-47.7(3)$ \\
\hline $\mathrm{C}(9)-\mathrm{C}(10)-\mathrm{C}(11)-\mathrm{C}(18)$ & $-54.0(3)$ \\
\hline$C(1)-C(10)-C(11)-C(18)$ & $75.9(3)$ \\
\hline$C(9)-C(10)-C(11)-C(19)$ & $67.7(3)$ \\
\hline$C(1)-C(10)-C(11)-C(19)$ & $-162.4(2)$ \\
\hline $\mathrm{C}(20)-\mathrm{O}(3)-\mathrm{C}(12)-\mathrm{C}(13)$ & $-29.2(2)$ \\
\hline $\mathrm{C}(20)-\mathrm{O}(3)-\mathrm{C}(12)-\mathrm{C}(11)$ & $-152.4(2)$ \\
\hline $\mathrm{C}(18)-\mathrm{C}(11)-\mathrm{C}(12)-\mathrm{O}(3)$ & $47.9(3)$ \\
\hline $\mathrm{C}(19)-\mathrm{C}(11)-\mathrm{C}(12)-\mathrm{O}(3)$ & $-73.1(2)$ \\
\hline $\mathrm{C}(10)-\mathrm{C}(11)-\mathrm{C}(12)-\mathrm{O}(3)$ & $171.93(19)$ \\
\hline $\mathrm{C}(18)-\mathrm{C}(11)-\mathrm{C}(12)-\mathrm{C}(13)$ & $-69.1(2)$ \\
\hline$C(19)-C(11)-C(12)-C(13)$ & $169.9(2)$ \\
\hline $\mathrm{C}(10)-\mathrm{C}(11)-\mathrm{C}(12)-\mathrm{C}(13)$ & $55.0(3)$ \\
\hline $\mathrm{C}(20)-\mathrm{O}(4)-\mathrm{C}(13)-\mathrm{C}(12)$ & $-41.4(2)$ \\
\hline $\mathrm{C}(20)-\mathrm{O}(4)-\mathrm{C}(13)-\mathrm{C}(14)$ & $-161.7(2)$ \\
\hline $\mathrm{O}(3)-\mathrm{C}(12)-\mathrm{C}(13)-\mathrm{O}(4)$ & $43.7(2)$ \\
\hline $\mathrm{C}(11)-\mathrm{C}(12)-\mathrm{C}(13)-\mathrm{O}(4)$ & $167.75(18)$ \\
\hline $\mathrm{O}(3)-\mathrm{C}(12)-\mathrm{C}(13)-\mathrm{C}(14)$ & $167.50(18)$ \\
\hline $\mathrm{C}(11)-\mathrm{C}(12)-\mathrm{C}(13)-\mathrm{C}(14)$ & $-68.4(3)$ \\
\hline $\mathrm{O}(4)-\mathrm{C}(13)-\mathrm{C}(14)-\mathrm{C}(1)$ & $178.5(2)$ \\
\hline $\mathrm{C}(12)-\mathrm{C}(13)-\mathrm{C}(14)-\mathrm{C}(1)$ & $64.0(2)$ \\
\hline $\mathrm{C}(15)-\mathrm{C}(1)-\mathrm{C}(14)-\mathrm{C}(13)$ & $67.4(2)$ \\
\hline$C(2)-C(1)-C(14)-C(13)$ & $-176.40(19)$ \\
\hline $\mathrm{C}(10)-\mathrm{C}(1)-\mathrm{C}(14)-\mathrm{C}(13)$ & $-53.7(2)$ \\
\hline $\mathrm{C}(12)-\mathrm{O}(3)-\mathrm{C}(20)-\mathrm{O}(4)$ & $4.0(3)$ \\
\hline $\mathrm{C}(12)-\mathrm{O}(3)-\mathrm{C}(20)-\mathrm{C}(21)$ & $122.9(2)$ \\
\hline $\mathrm{C}(12)-\mathrm{O}(3)-\mathrm{C}(20)-\mathrm{C}(22)$ & $-113.1(2)$ \\
\hline $\mathrm{C}(13)-\mathrm{O}(4)-\mathrm{C}(20)-\mathrm{O}(3)$ & $24.2(3)$ \\
\hline $\mathrm{C}(13)-\mathrm{O}(4)-\mathrm{C}(20)-\mathrm{C}(21)$ & $-93.1(2)$ \\
\hline $\mathrm{C}(13)-\mathrm{O}(4)-\mathrm{C}(20)-\mathrm{C}(22)$ & $141.7(2)$ \\
\hline
\end{tabular}


1D and 2D NMR Spectra 


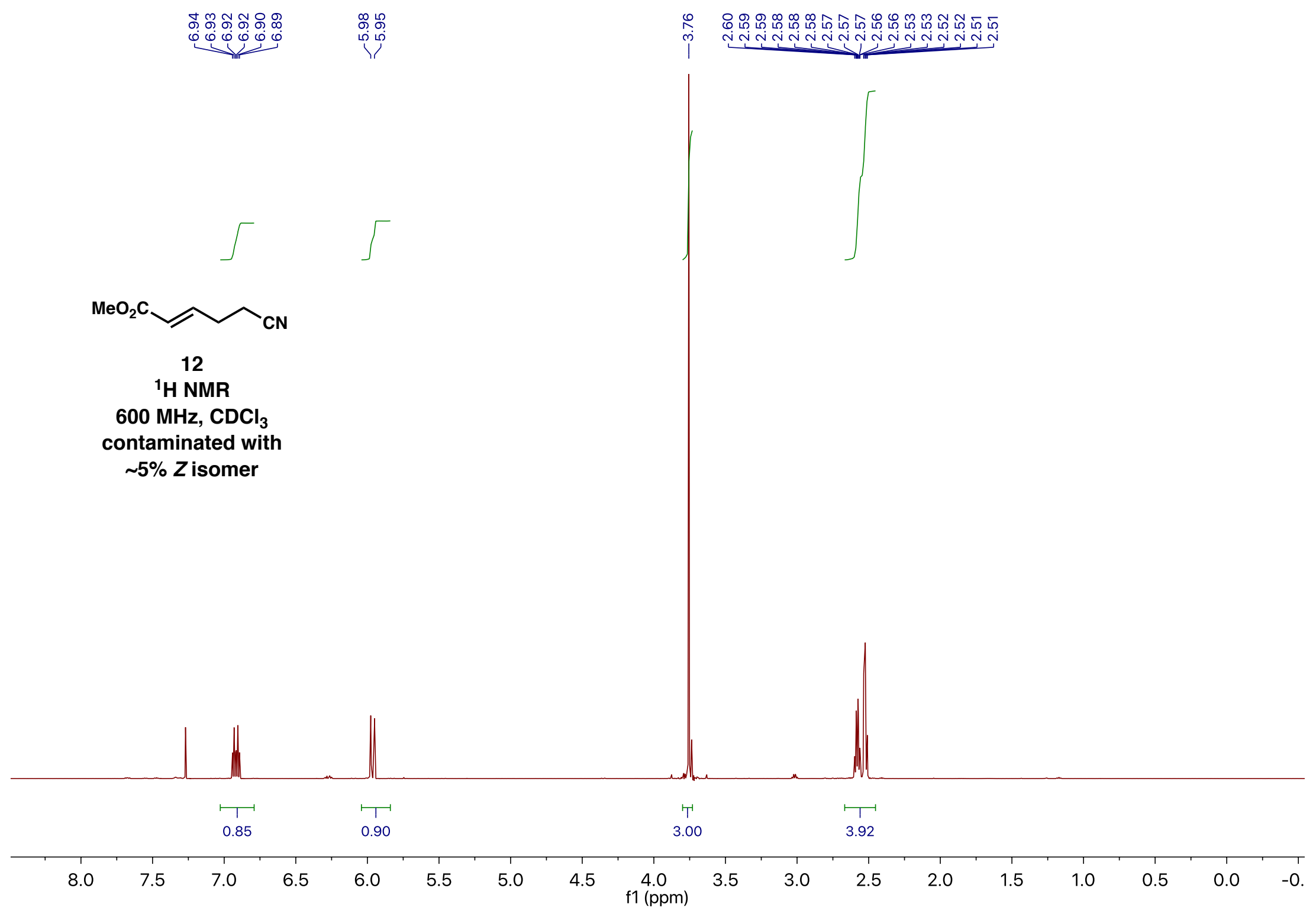




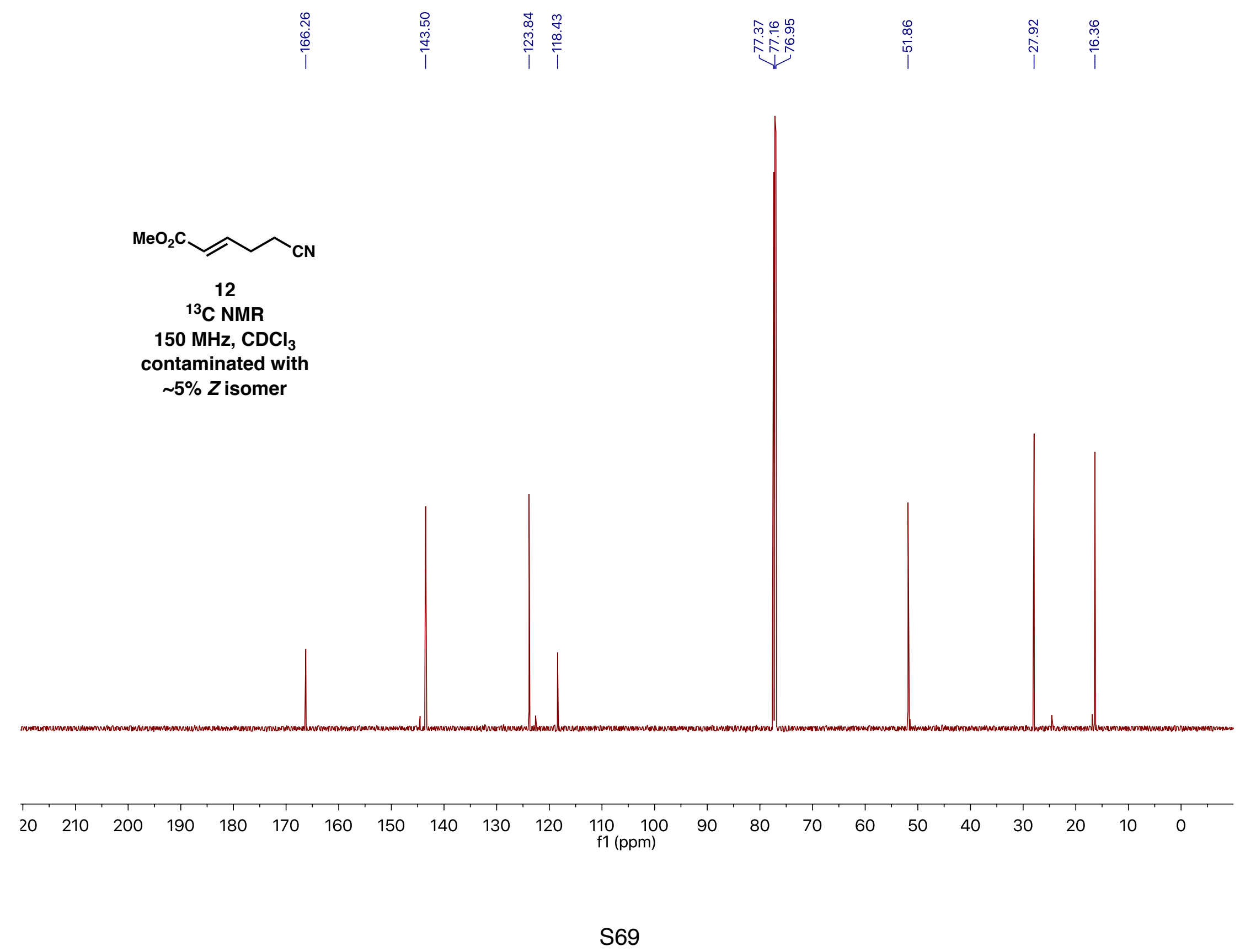




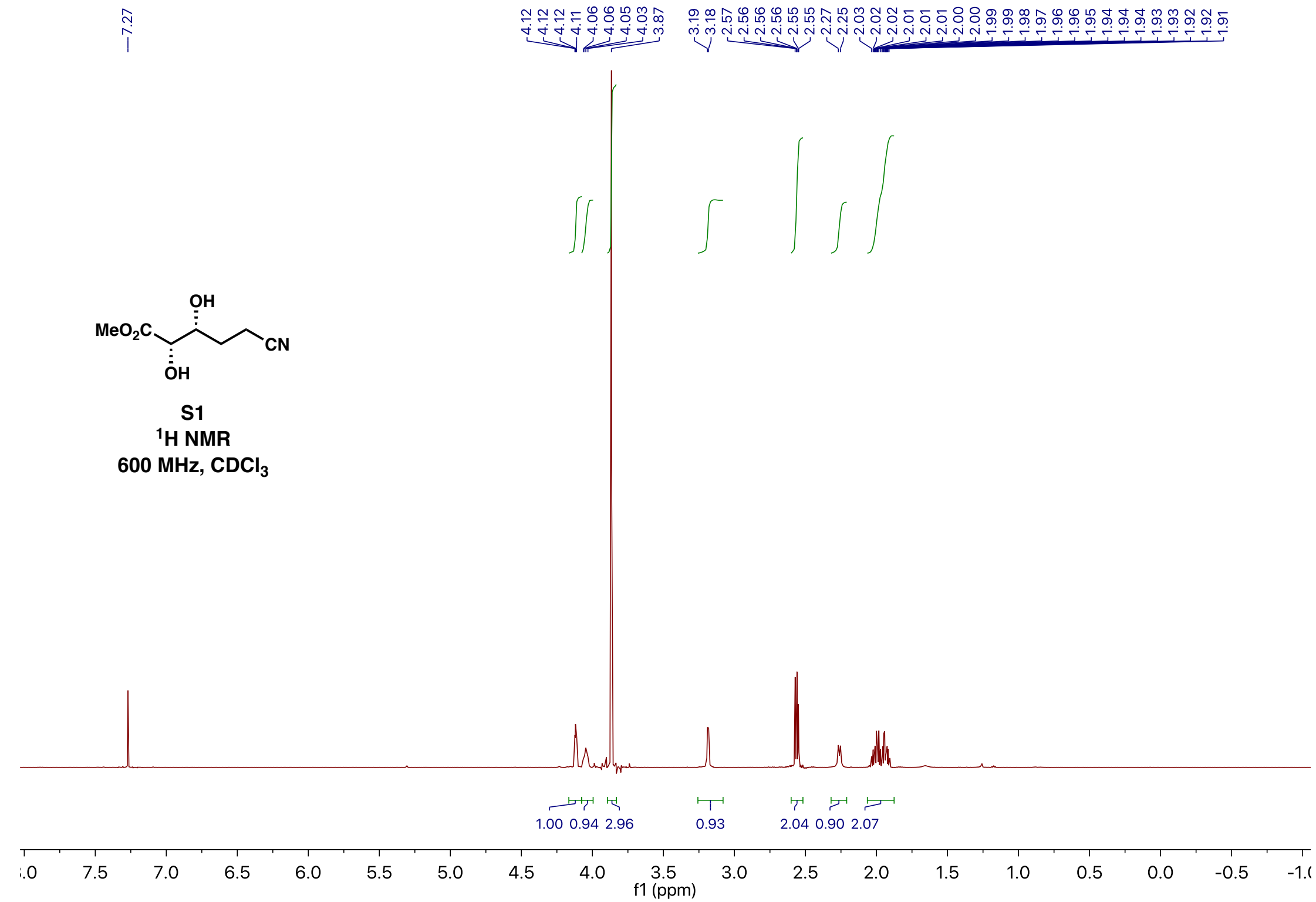




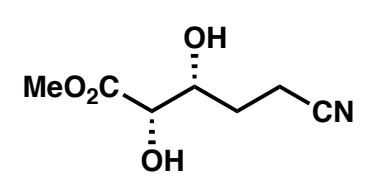

S1

${ }^{13} \mathrm{C}$ NMR

$150 \mathrm{MHz}, \mathrm{CDCl}_{3}$

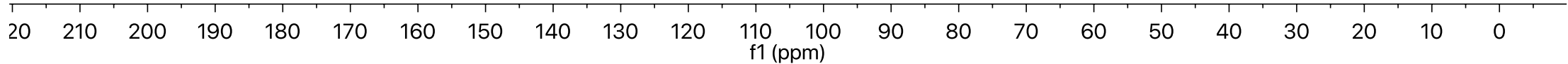




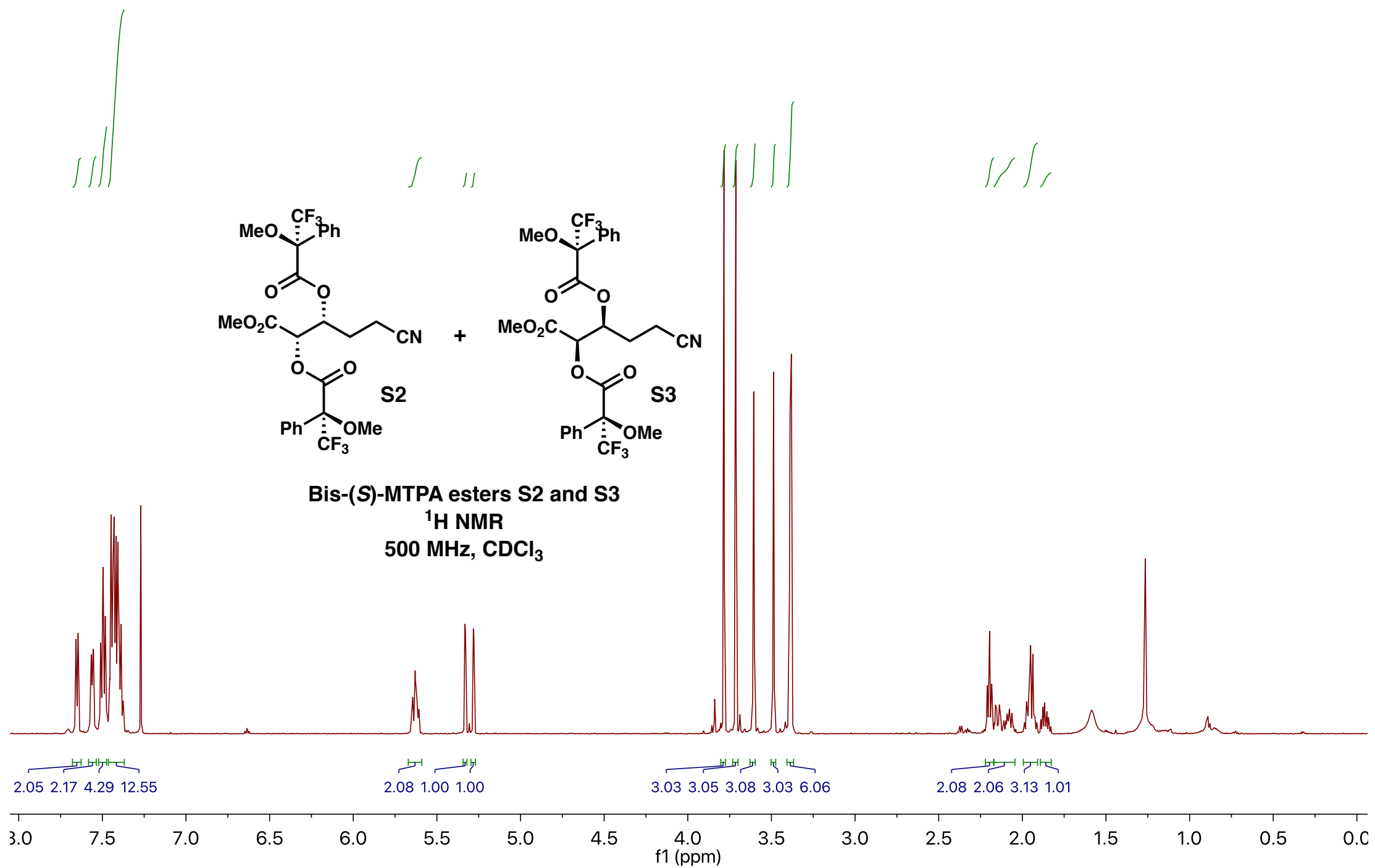




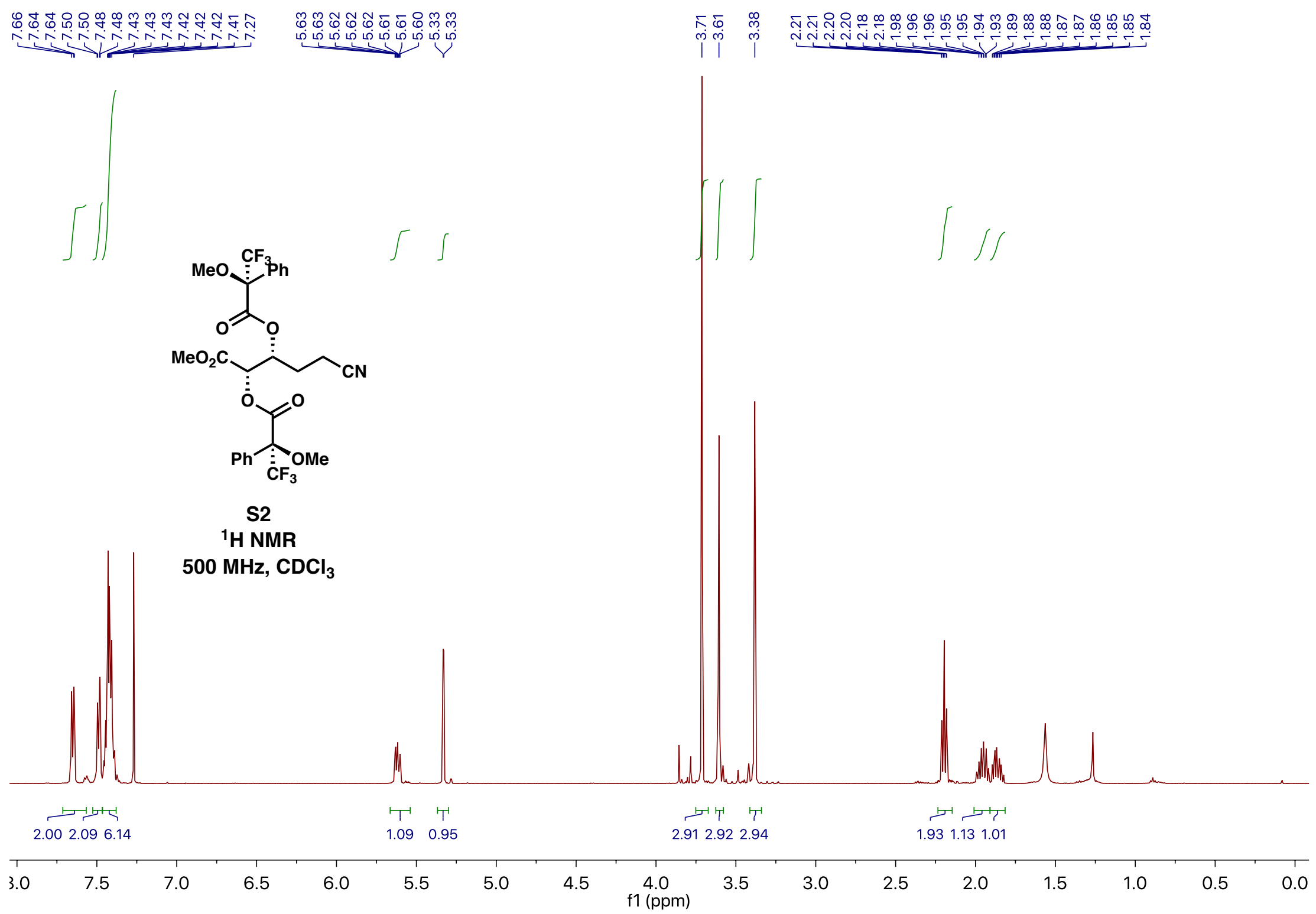




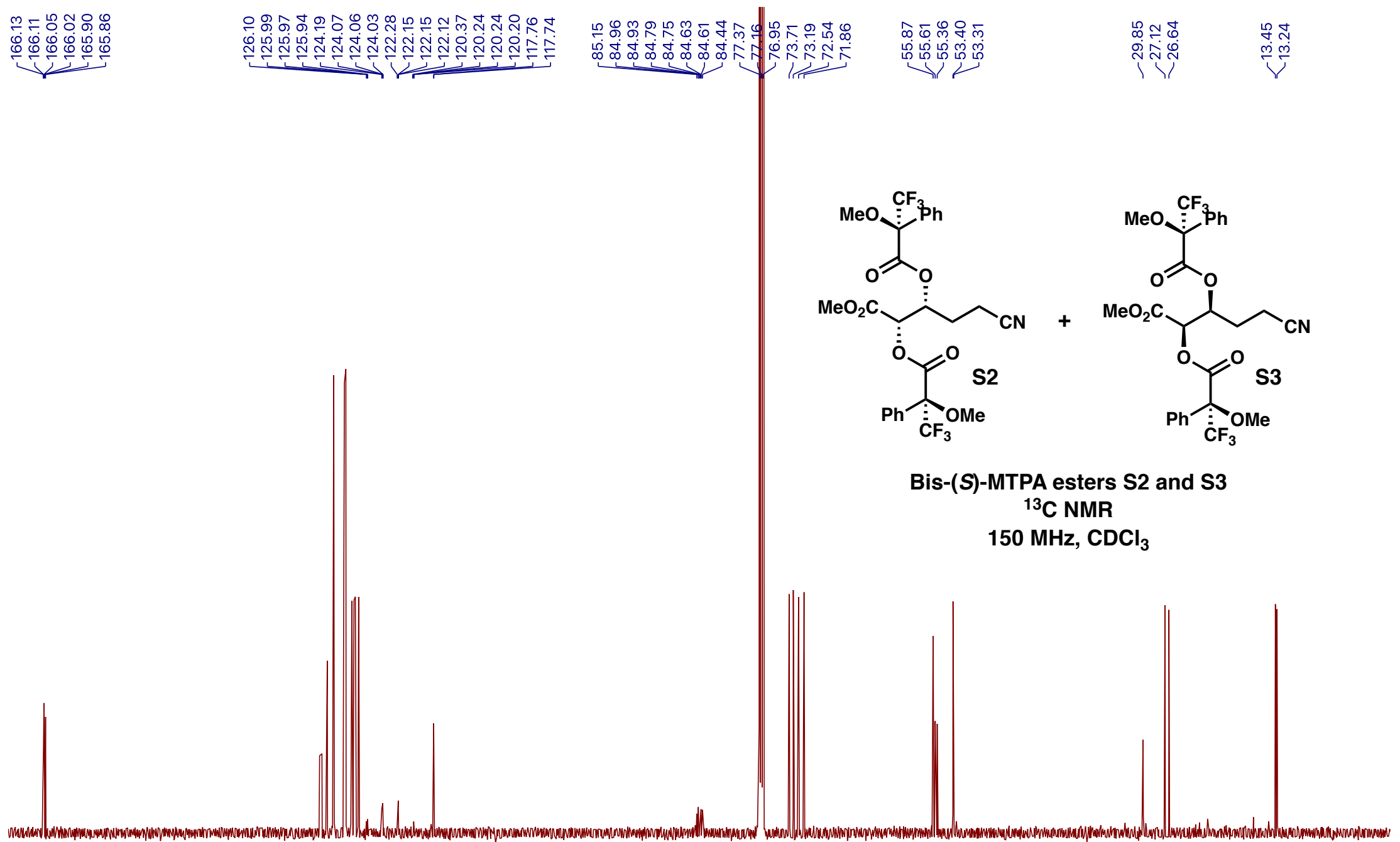

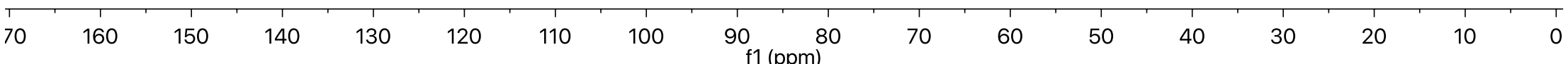




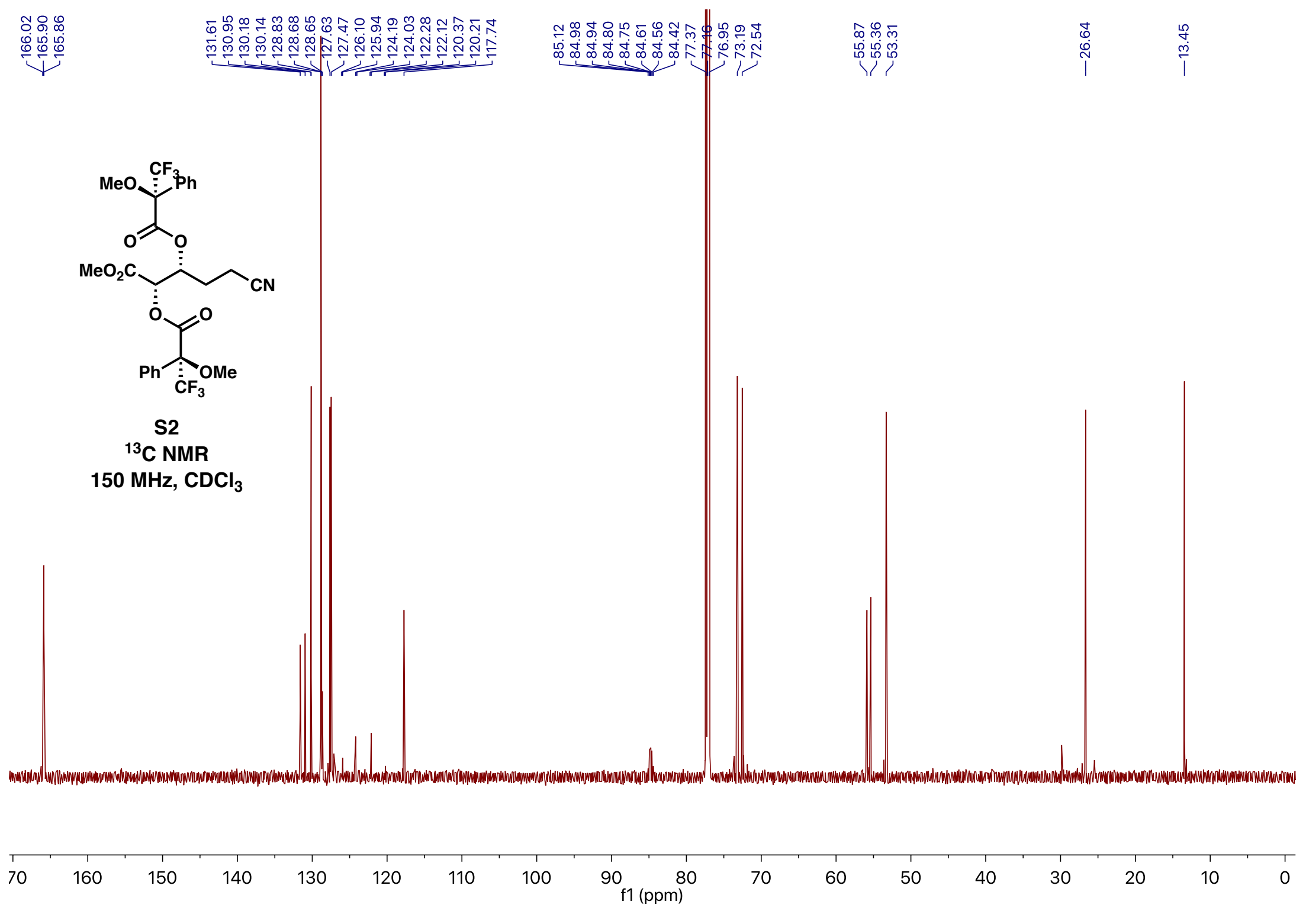




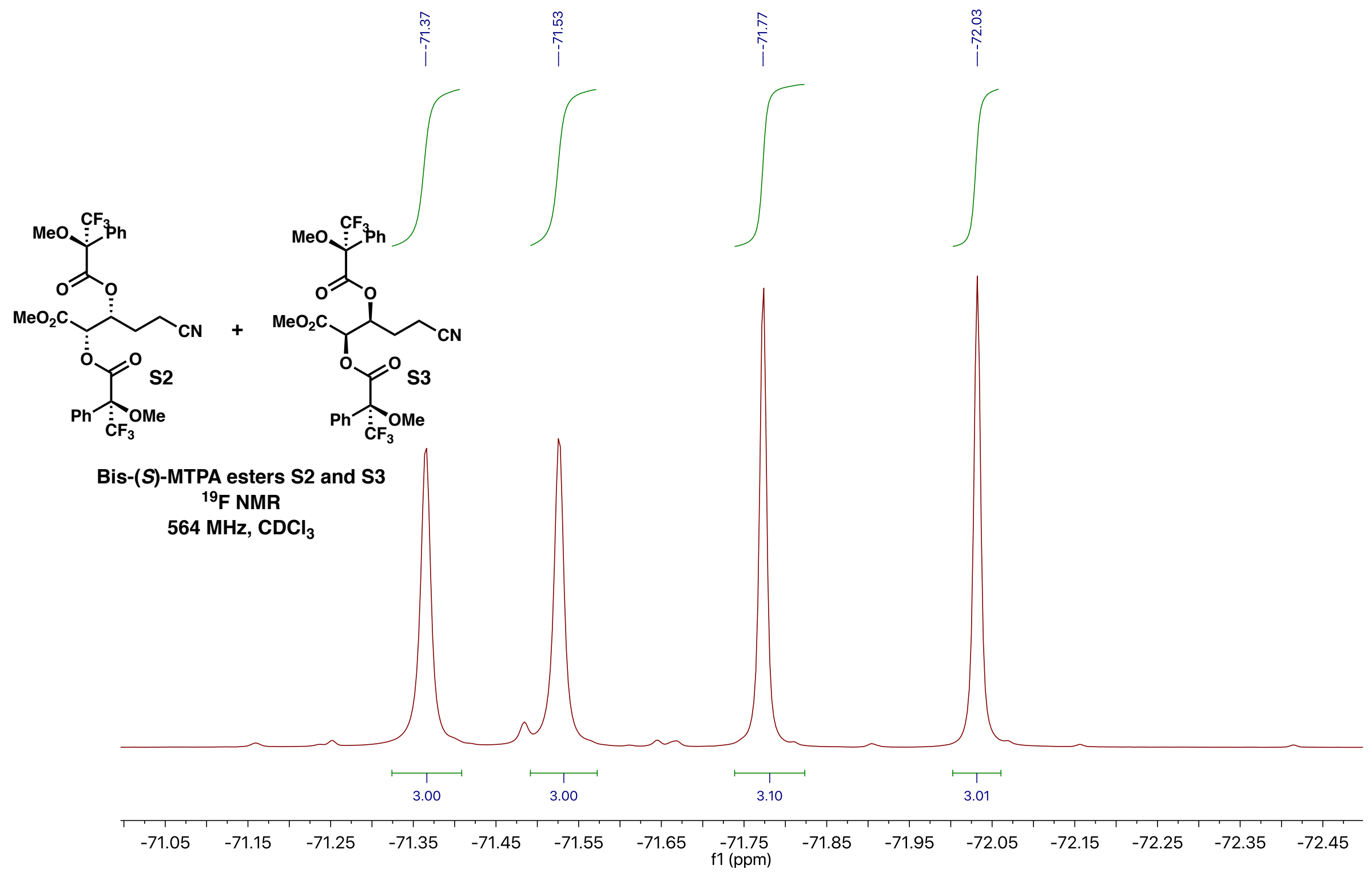




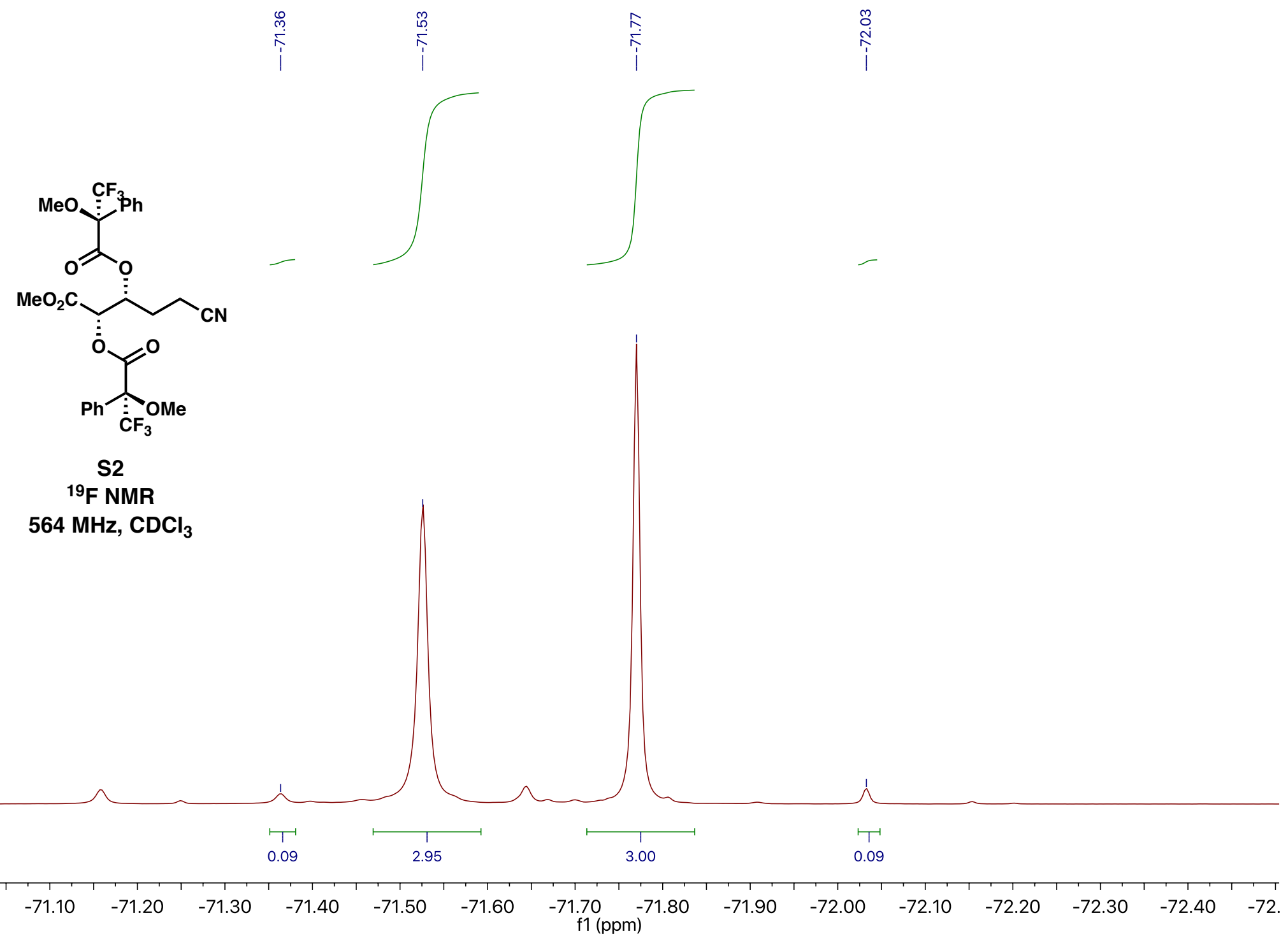




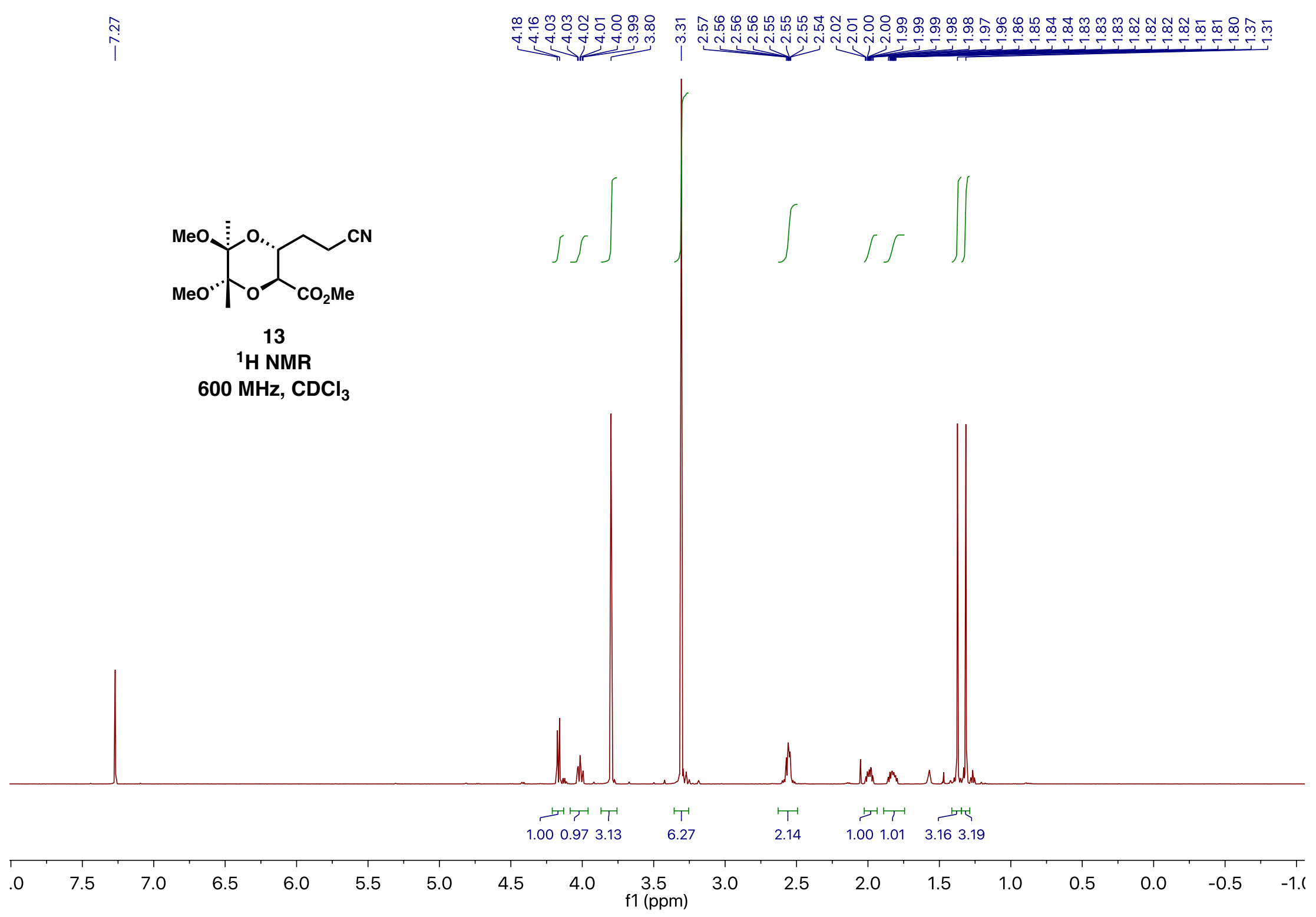




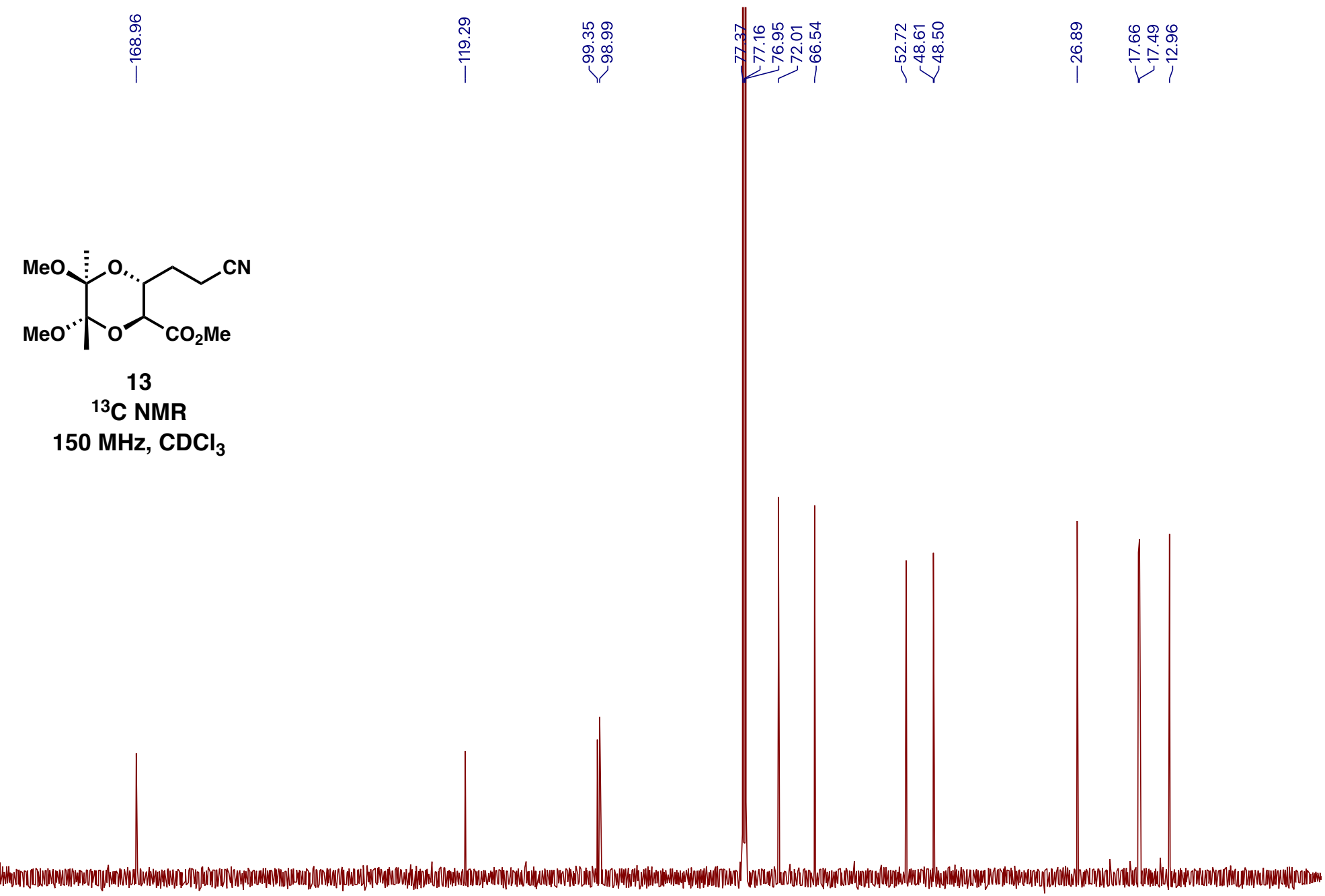

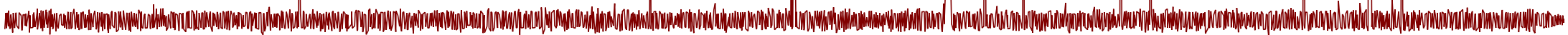

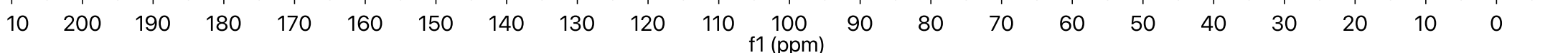




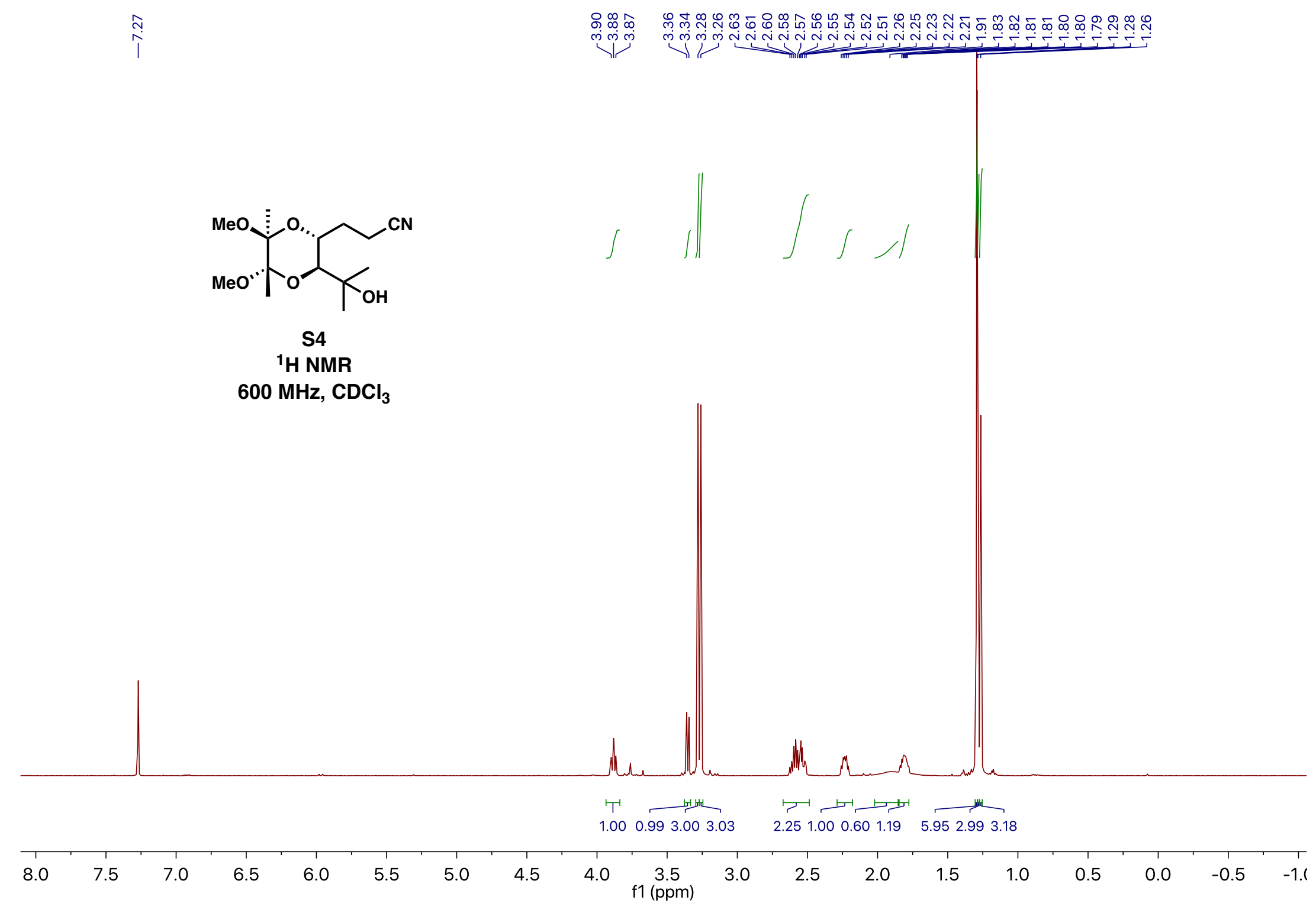




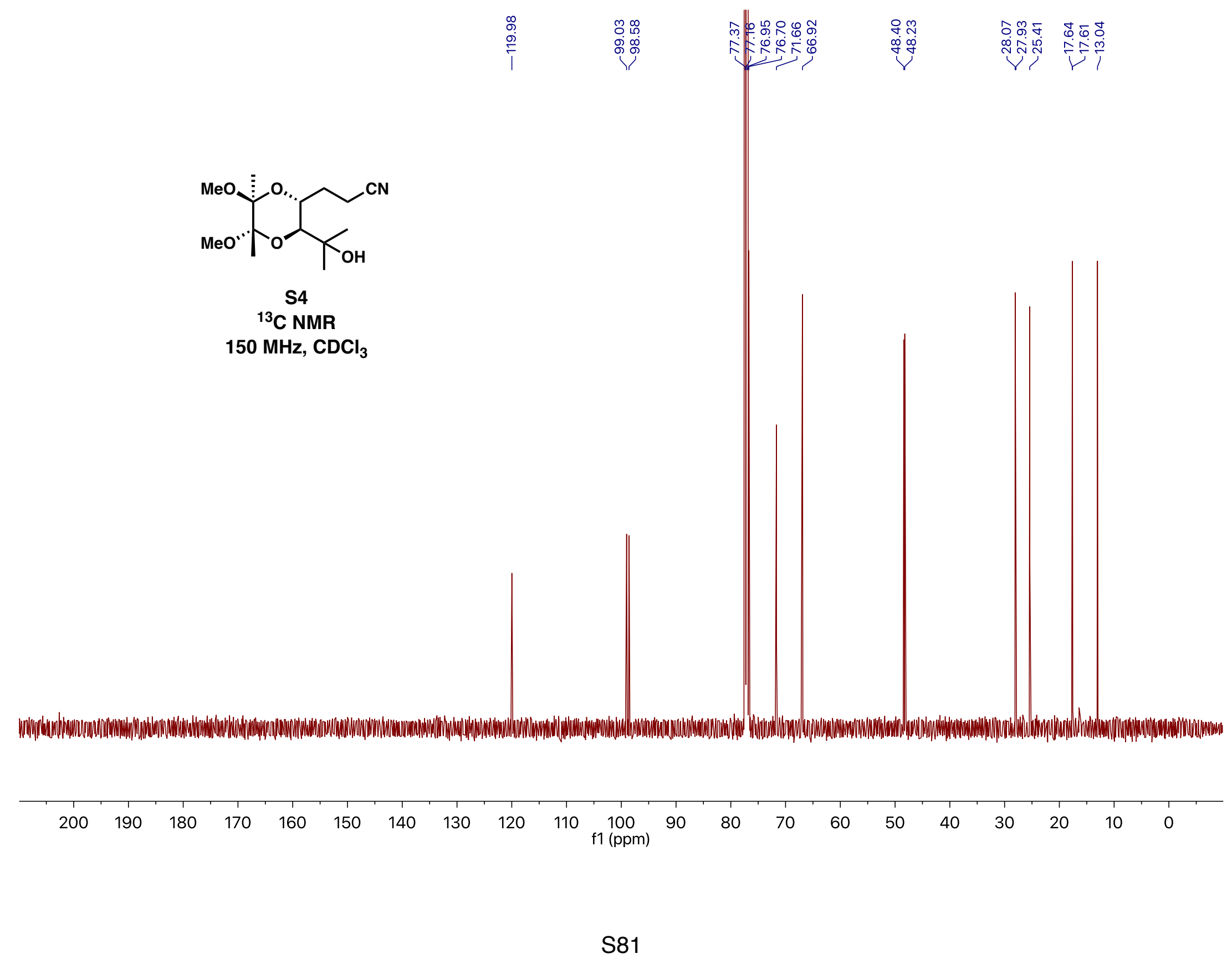




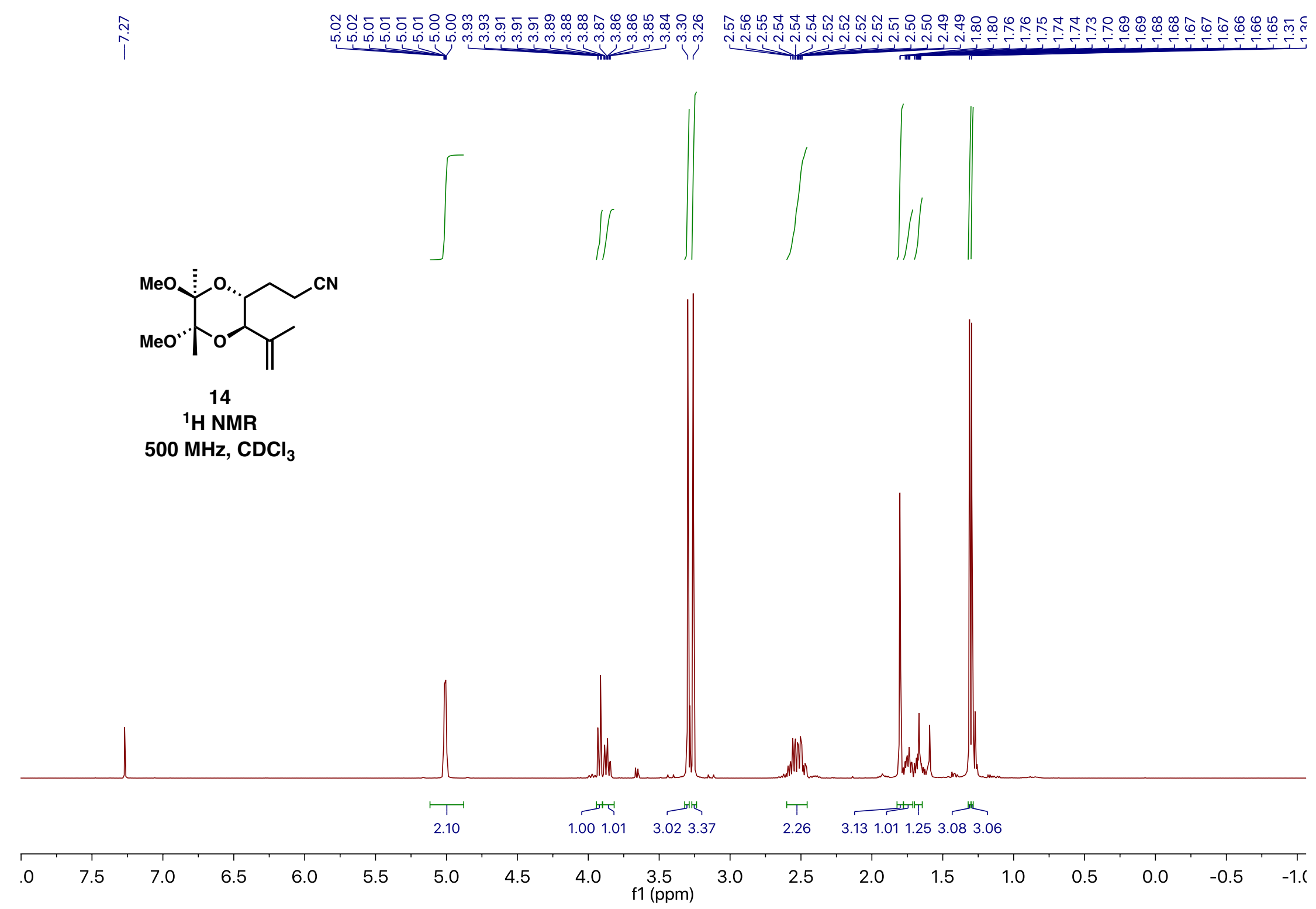




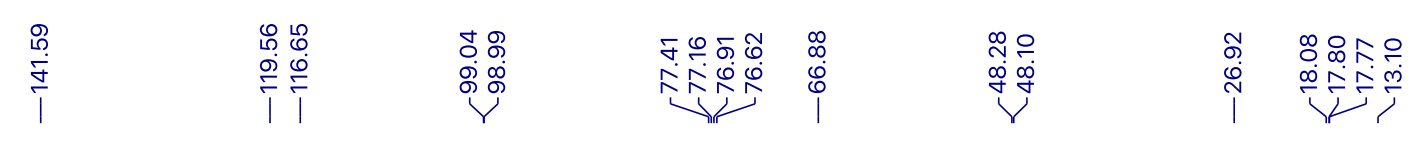

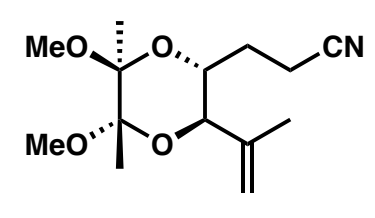

14

${ }^{13} \mathrm{C}$ NMR

$125 \mathrm{MHz}, \mathrm{CDCl}_{3}$

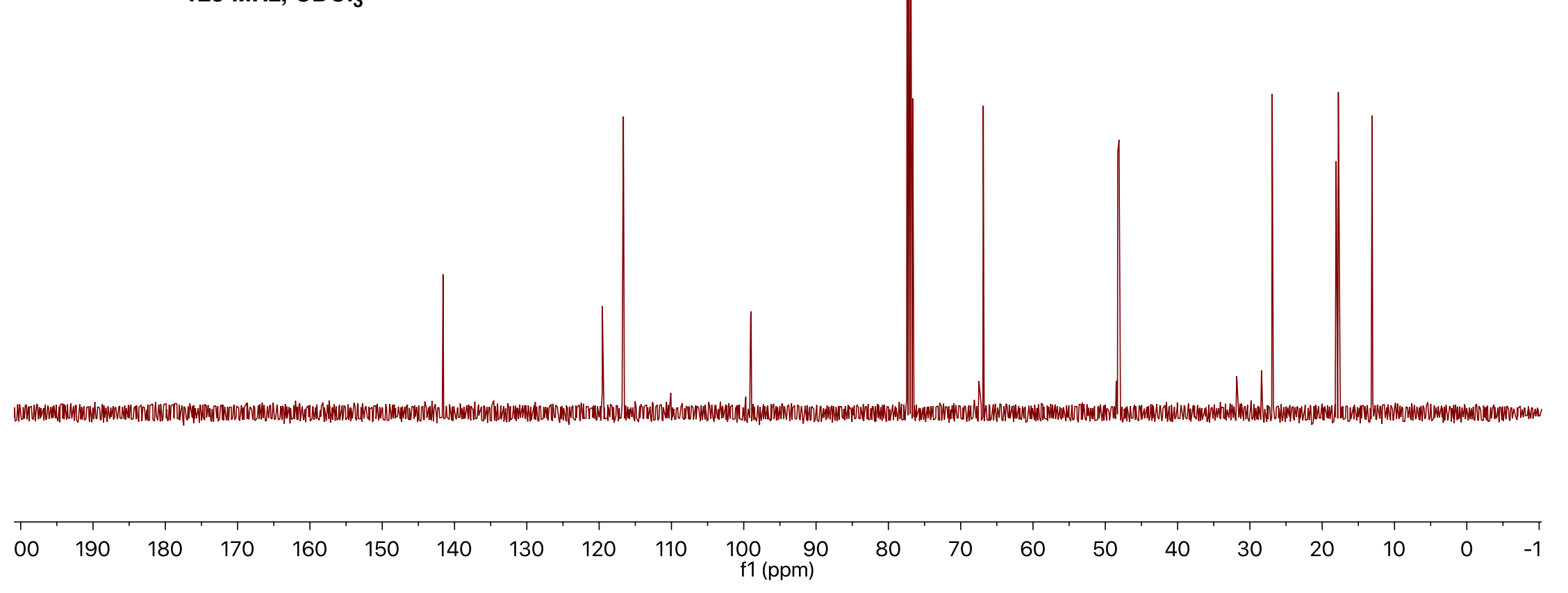




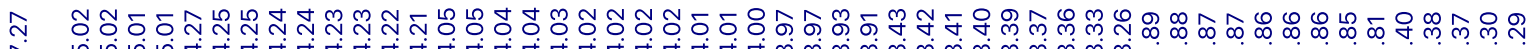

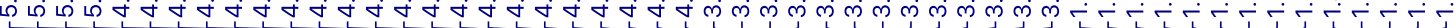

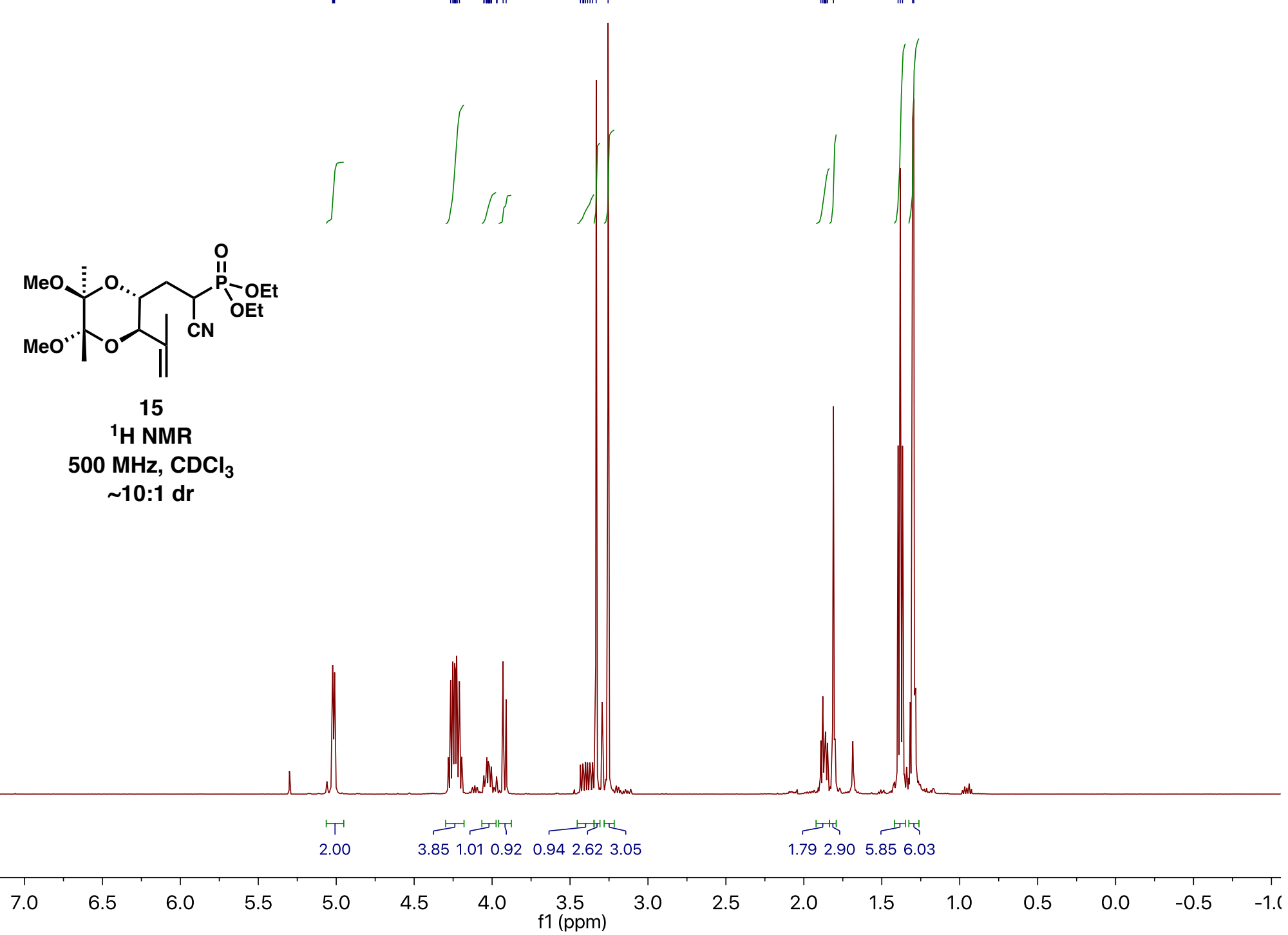




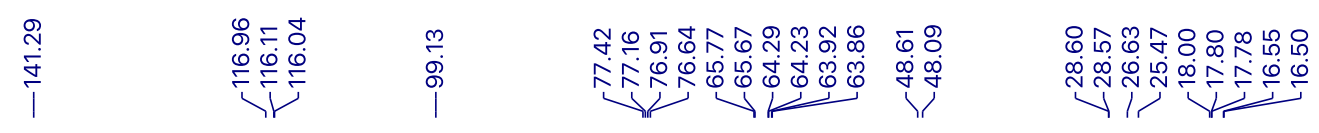

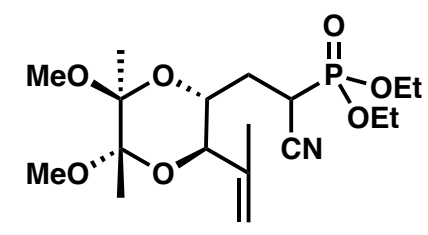

15

${ }^{13} \mathrm{C}$ NMR

$125 \mathrm{MHz}, \mathrm{CDCl}_{3}$

$\sim 10: 1 \mathrm{dr}$

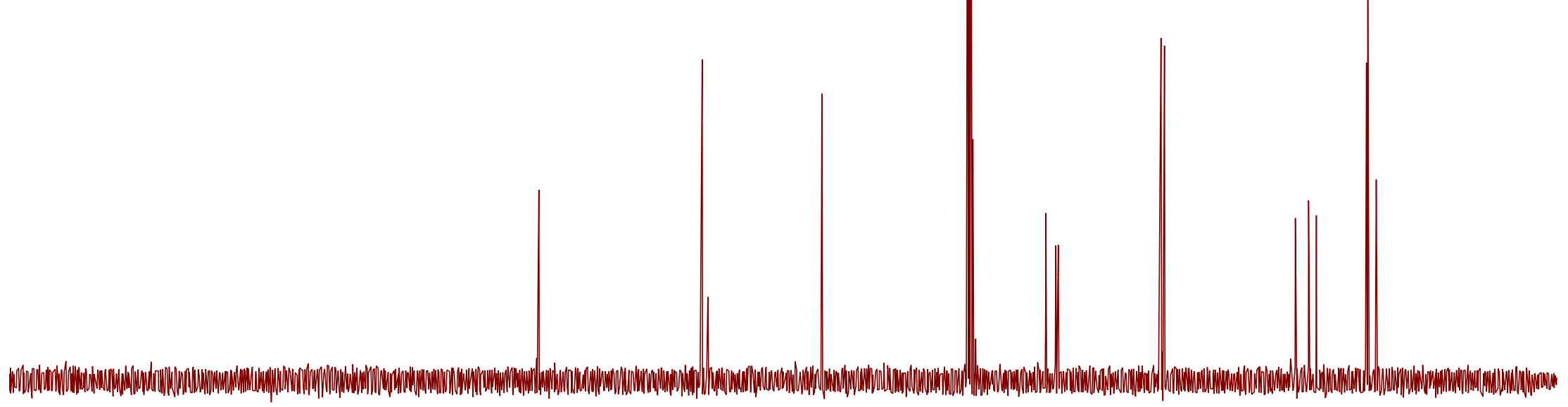

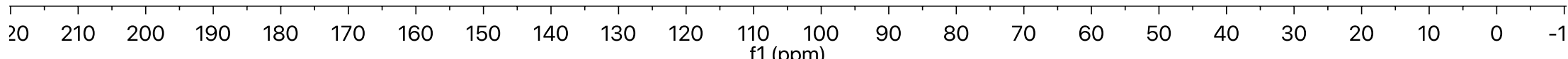




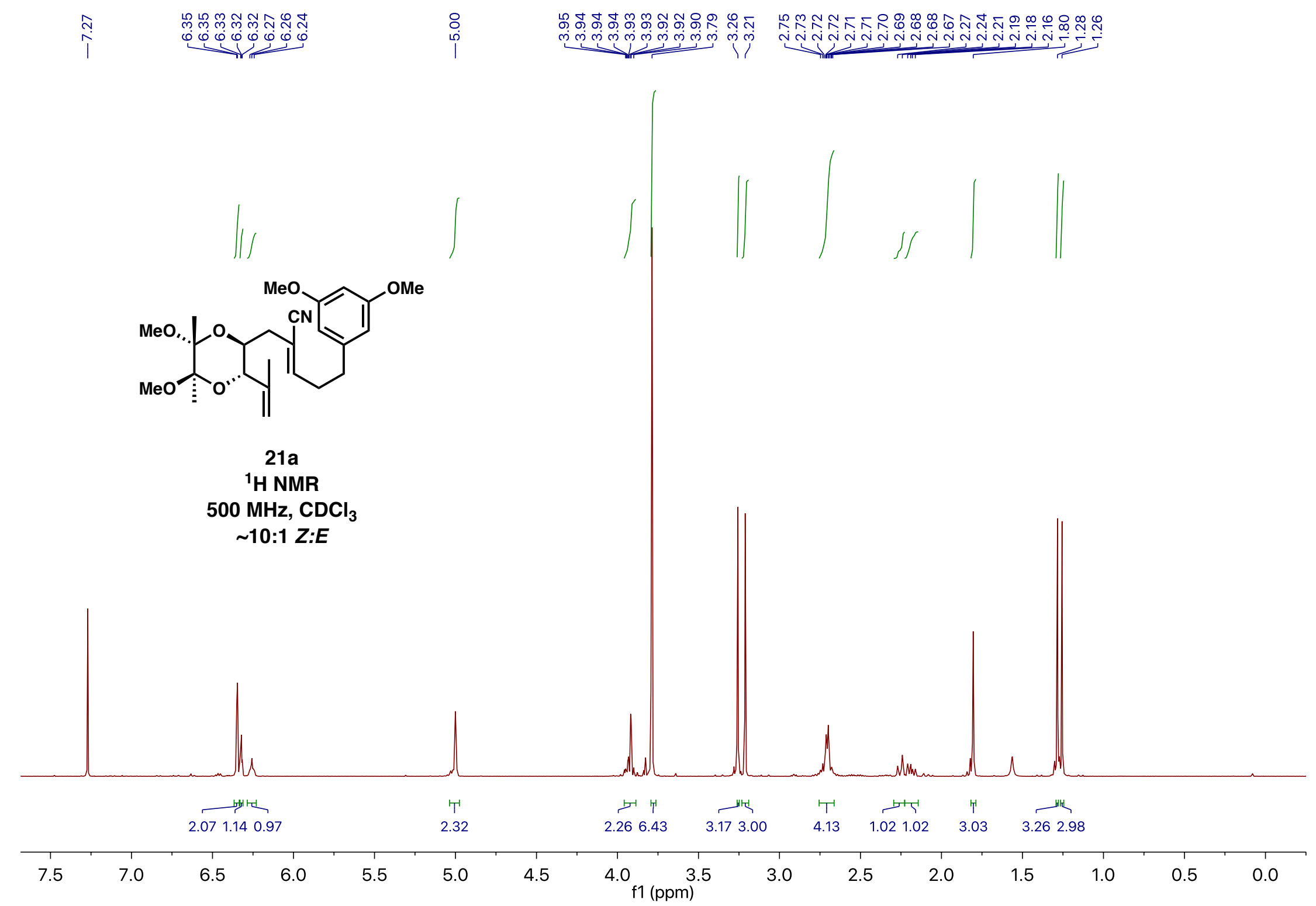




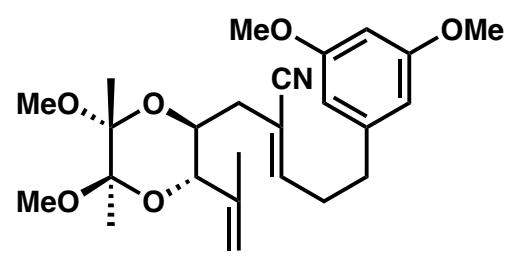

21a

${ }^{13} \mathrm{C}$ NMR

$125 \mathrm{MHz}, \mathrm{CDCl}_{3}$

$\sim 10: 1 \mathrm{Z:E}$

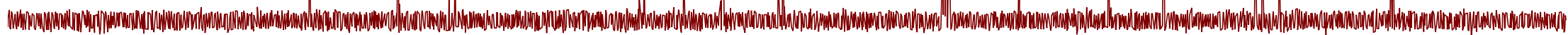

\begin{tabular}{lllllllllllllllllllllll}
\hline 00 & 190 & 180 & 170 & 160 & 150 & 140 & 130 & 120 & 110 & $\begin{array}{c}100 \\
\mathrm{f} 1(\mathrm{ppm})\end{array}$ & 90 & 80 & 70 & 60 & 50 & 40 & 30 & 20 & 10 & 0
\end{tabular}




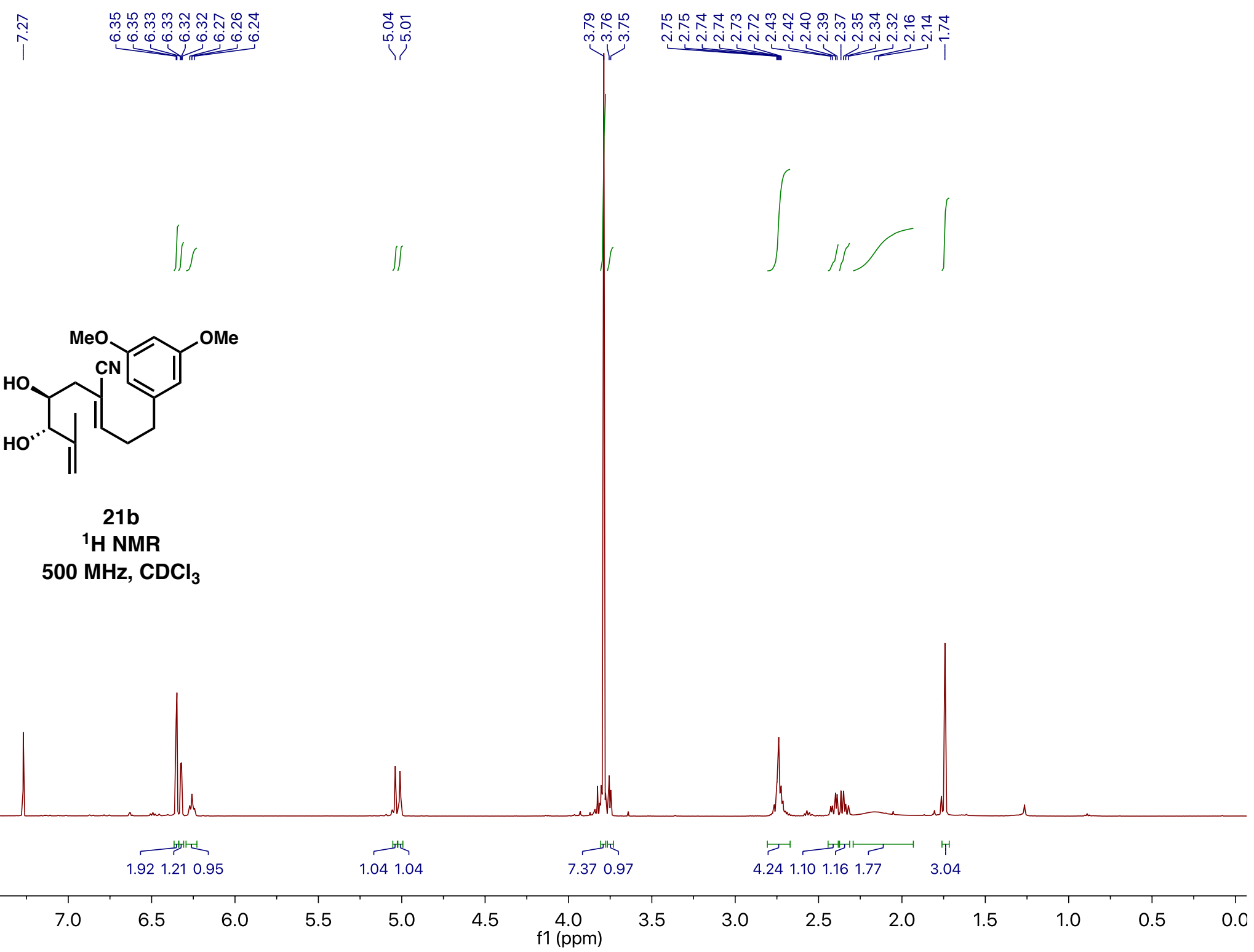




\begin{tabular}{|c|c|c|c|c|c|c|}
\hline 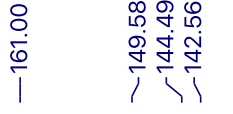 & 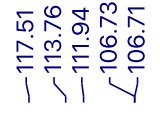 & $\begin{array}{c}\infty \\
\infty \\
\infty \\
\infty \\
\infty\end{array}$ & $\stackrel{0}{\stackrel{i}{i}}$ & $\begin{array}{l}\text { \% } \\
\dot{0} \\
0 \\
1\end{array}$ & 号 & 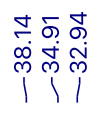 \\
\hline
\end{tabular}

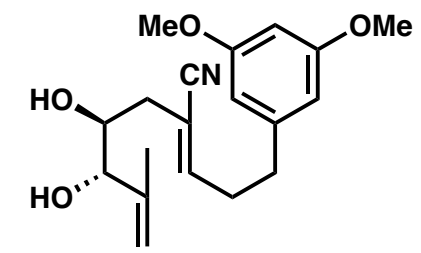

21b

${ }^{13} \mathrm{C}$ NMR

$125 \mathrm{MHz}, \mathrm{CDCl}_{3}$

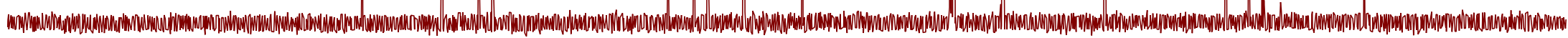

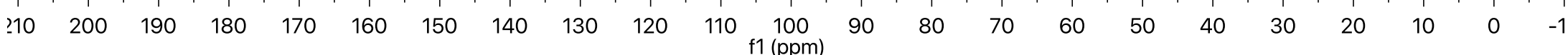




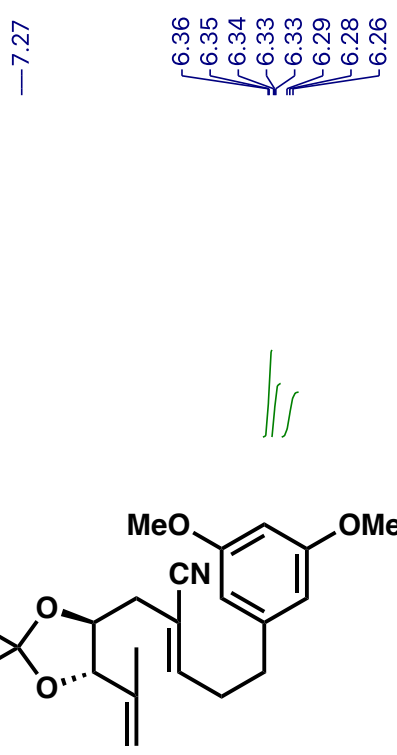

21c

${ }^{1} \mathrm{H}$ NMR

$600 \mathrm{MHz}, \mathrm{CDCl}_{3}$

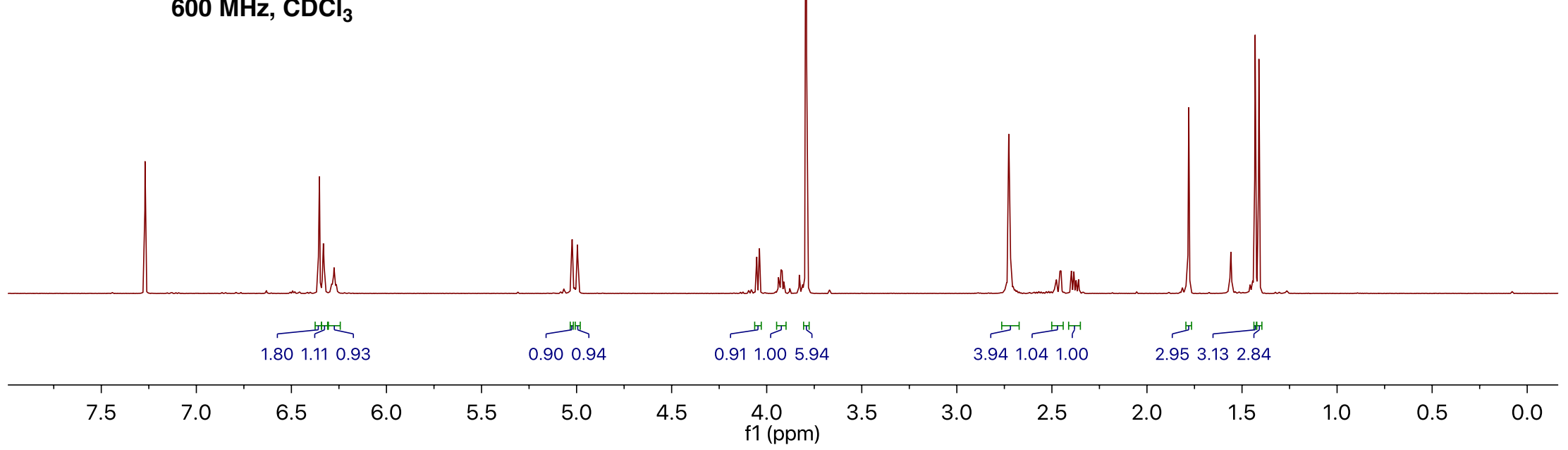



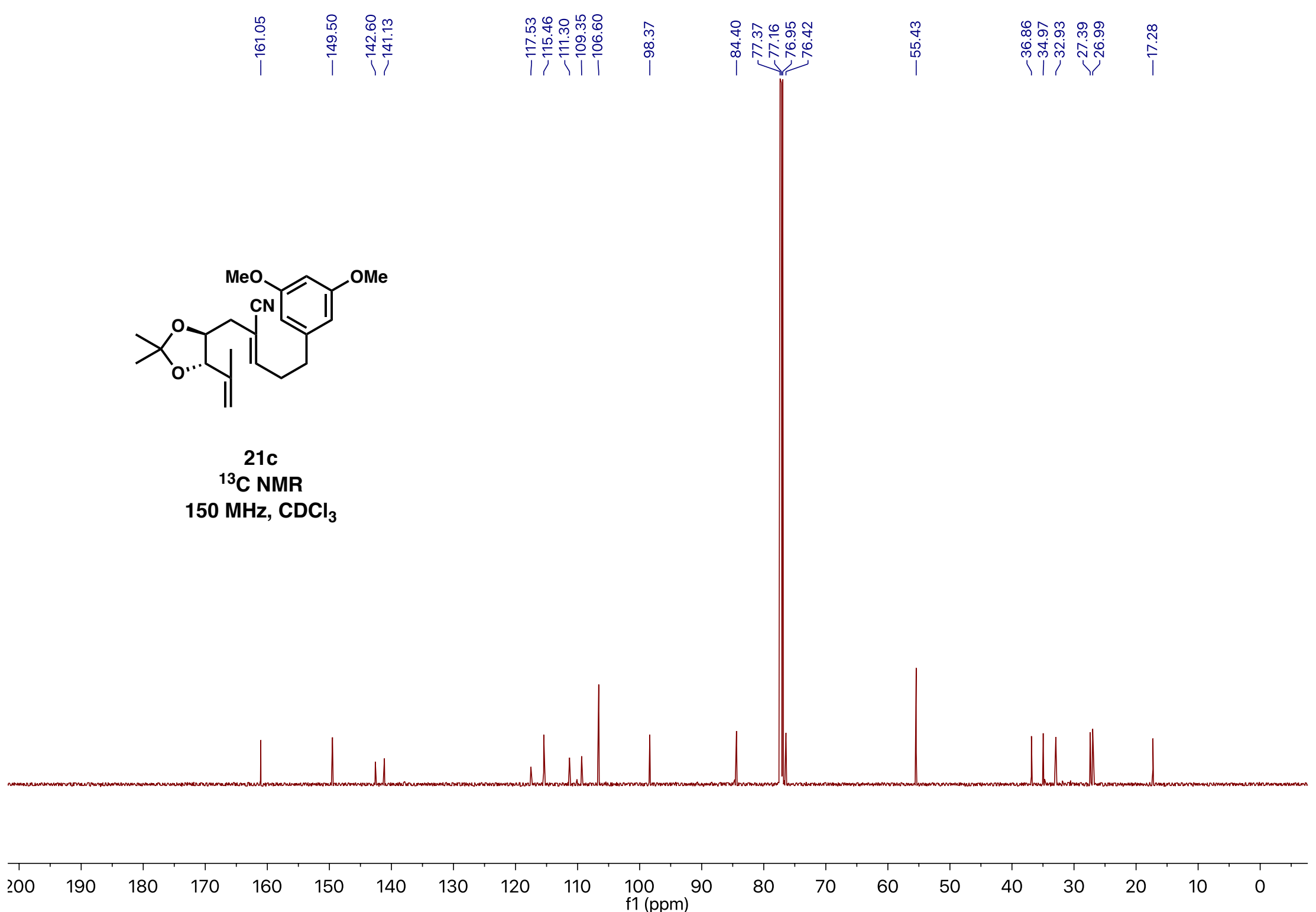


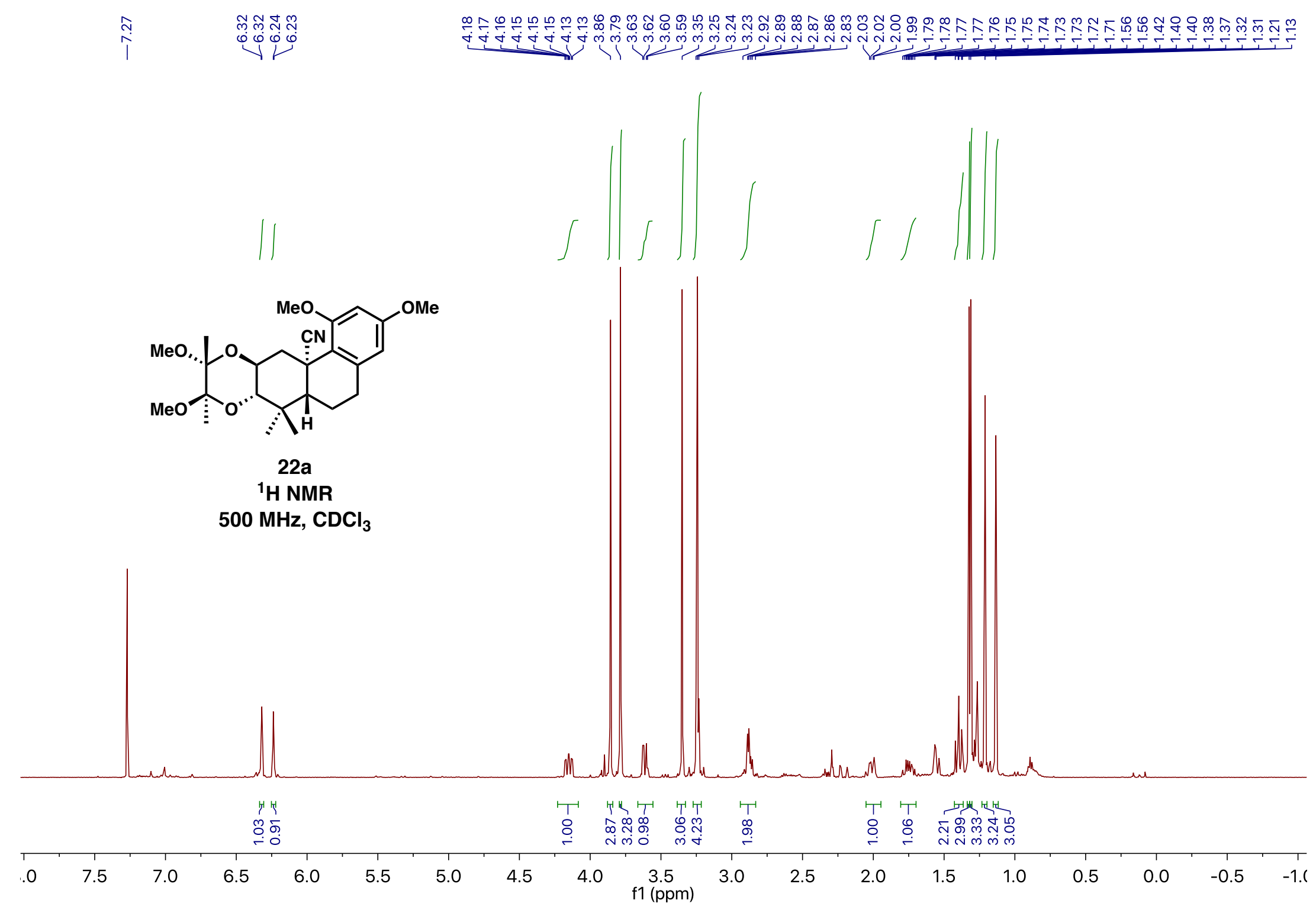




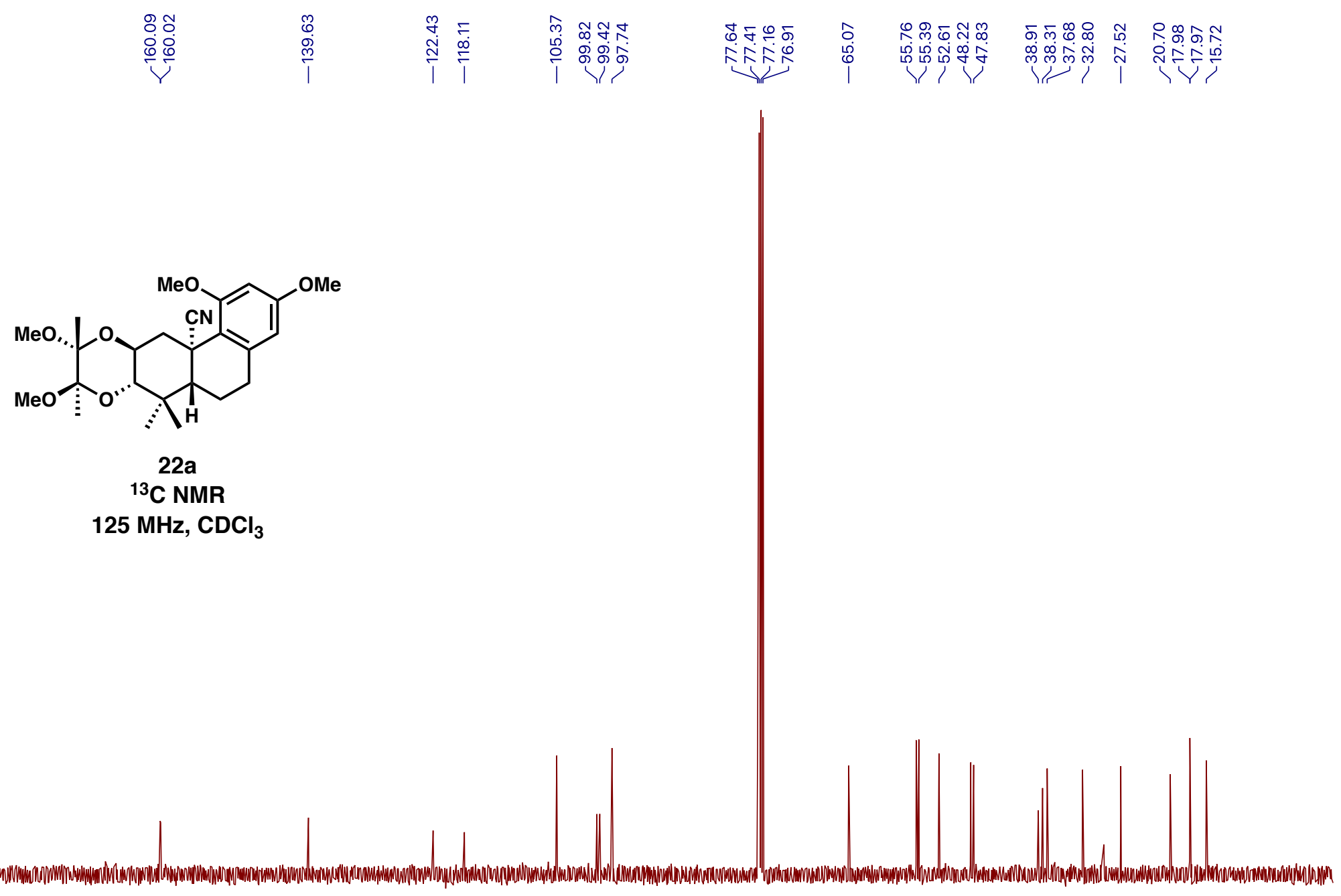

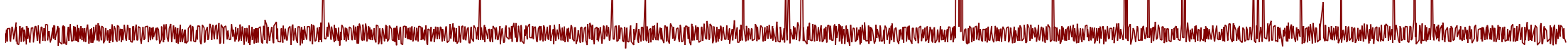

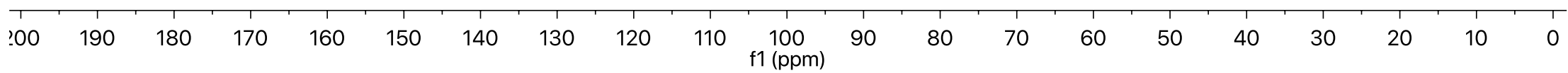




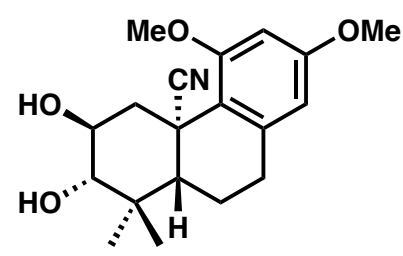

$22 b$

${ }^{1} \mathrm{H}$ NMR $500 \mathrm{MHz}, \mathrm{CDCl}_{3}$

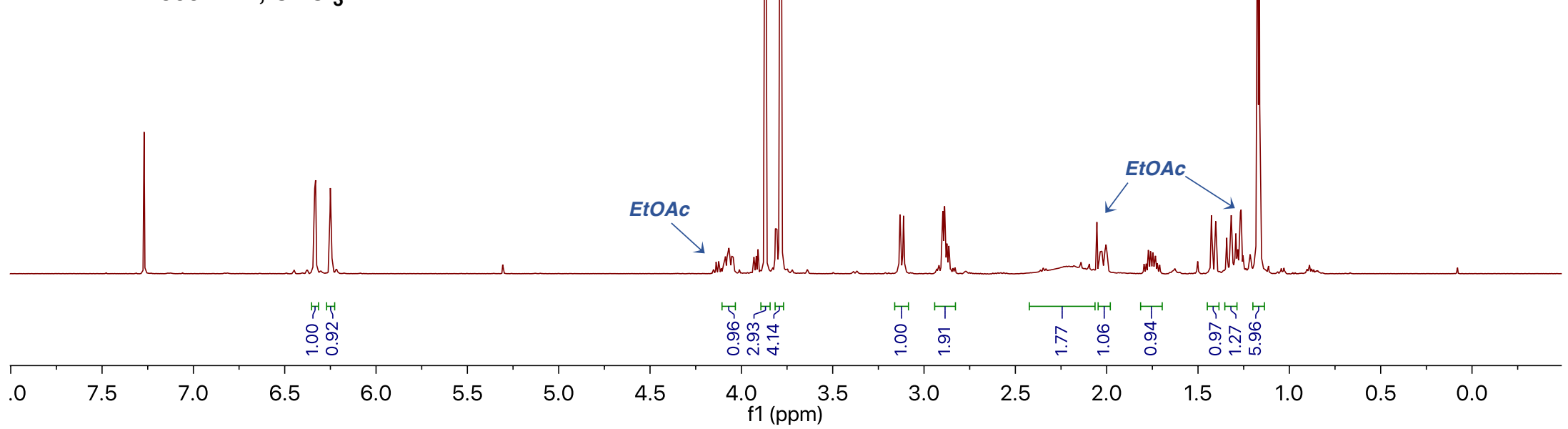




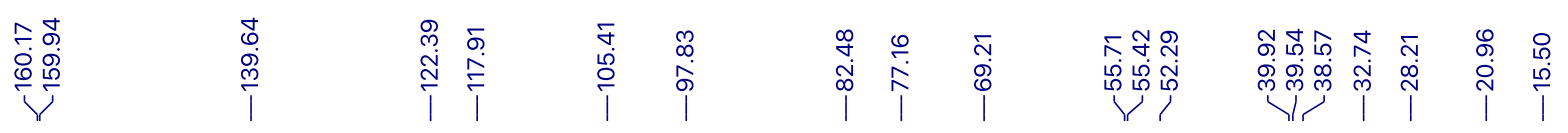

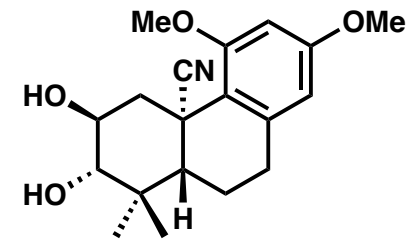

22b

${ }^{13} \mathrm{C}$ NMR

$125 \mathrm{MHz}, \mathrm{CDCl}_{3}$

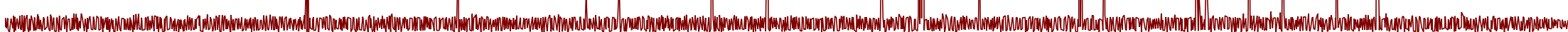

\begin{tabular}{lllllllllllllllllllllll}
\hline 00 & 190 & 180 & 170 & 160 & 150 & 140 & 130 & 120 & 110 & $\underset{f 1}{100}(\mathrm{ppm})$ & 90 & 80 & 70 & 60 & 50 & 40 & 30 & 20 & 10 & 0 & -1
\end{tabular}



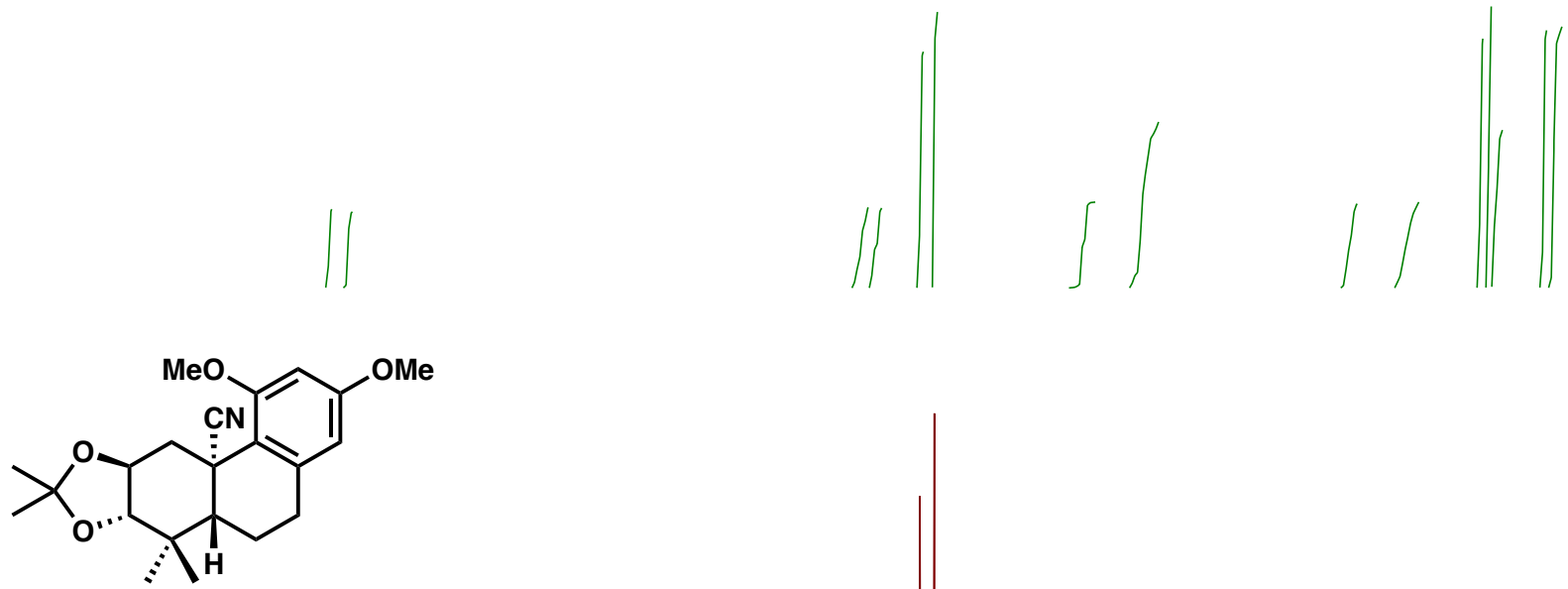

22c

${ }^{1} \mathrm{H}$ NMR $500 \mathrm{MHz}, \mathrm{CDCl}_{3}$ ca. $90 \%$ pure

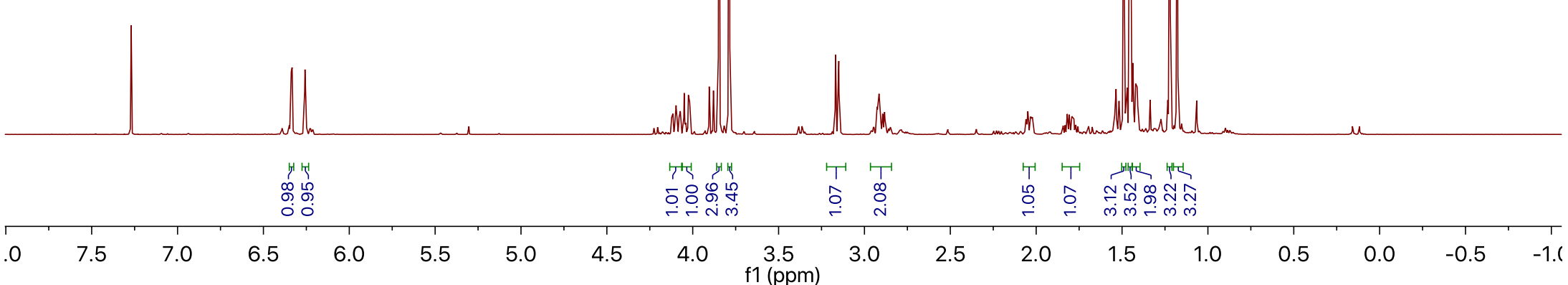




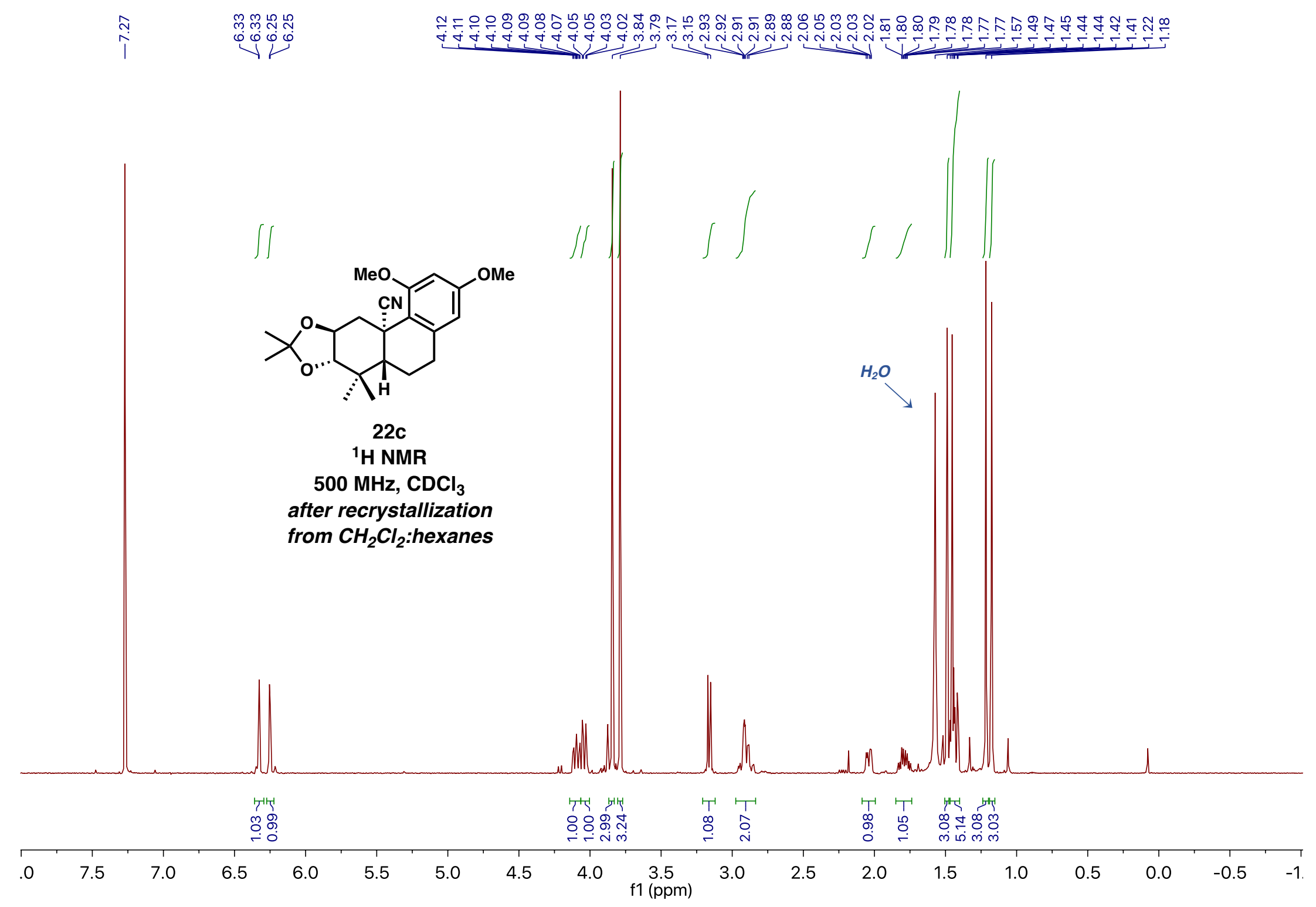




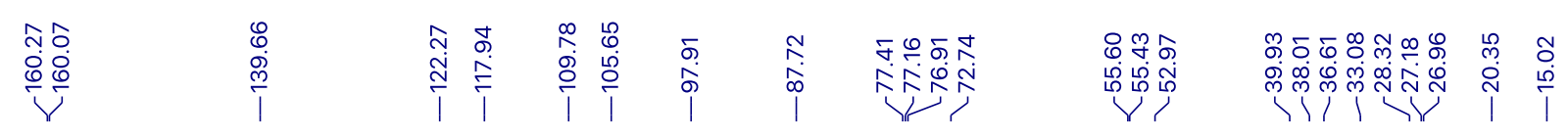

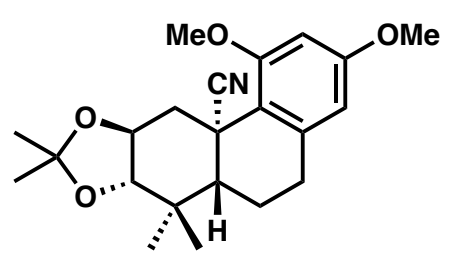

22c

${ }^{13} \mathrm{C}$ NMR

$125 \mathrm{MHz}, \mathrm{CDCl}_{3}$

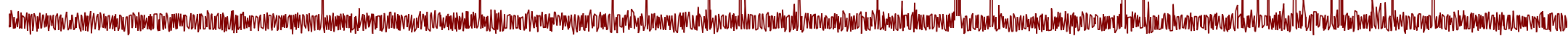

140

130

120

100

1 (ppm)

60

50

40

30

20

$10 \quad 0$ 


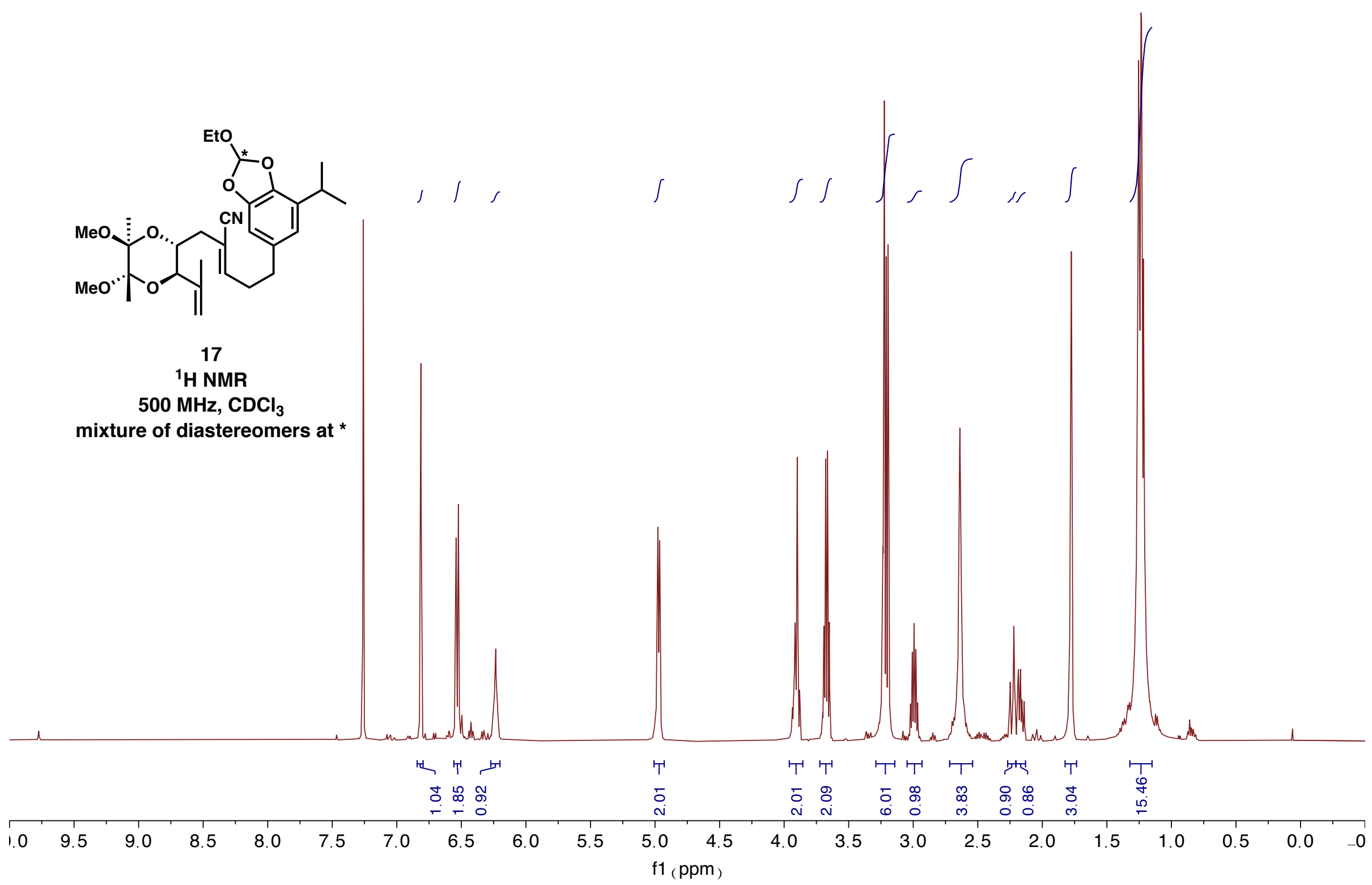


ำ 유

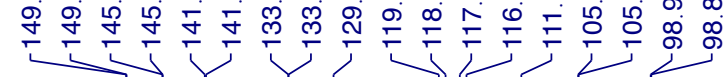

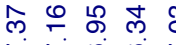
全企是
万 $8 \%$ \&

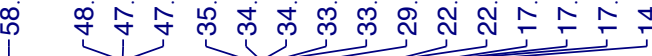

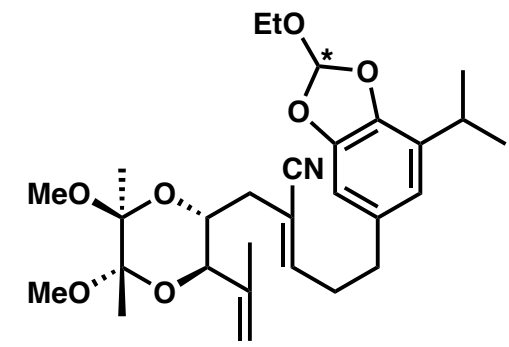

17

${ }^{13} \mathrm{C}$ NMR

$151 \mathrm{MHz}, \mathrm{CDCl}_{3}$

mixture of diastereomers at * 


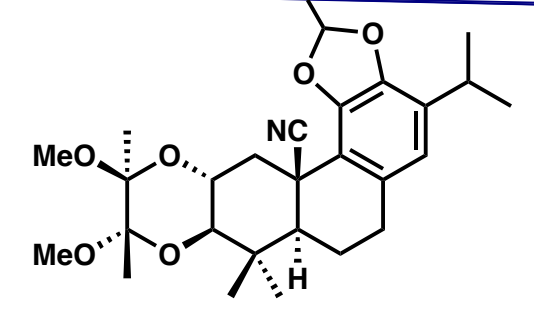

$18 \mathrm{a}$

${ }^{1} \mathrm{H}$ NMR

$600 \mathrm{MHz}, \mathrm{CDCl}_{3}$

more polar diastereomer
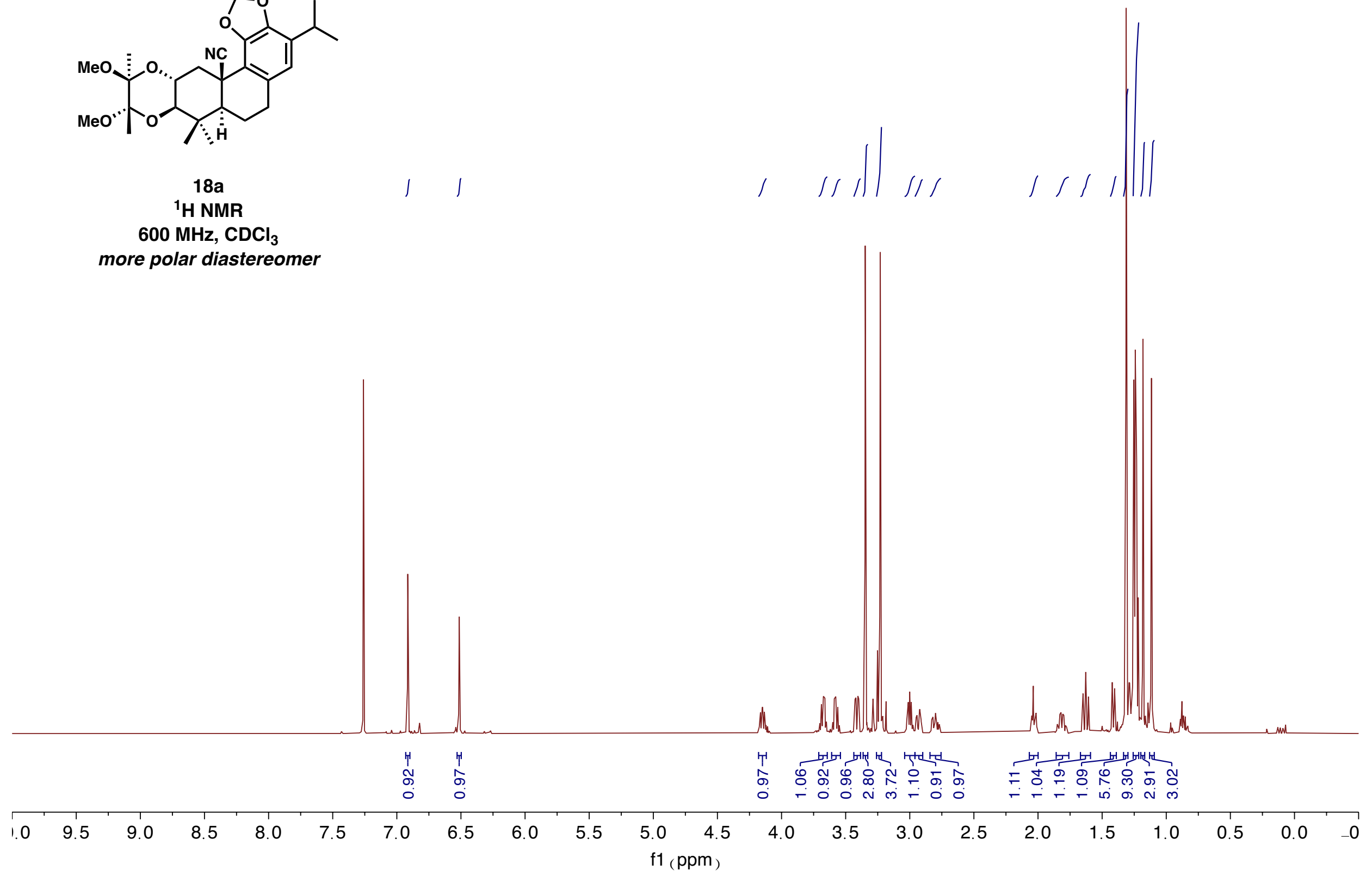

S101 


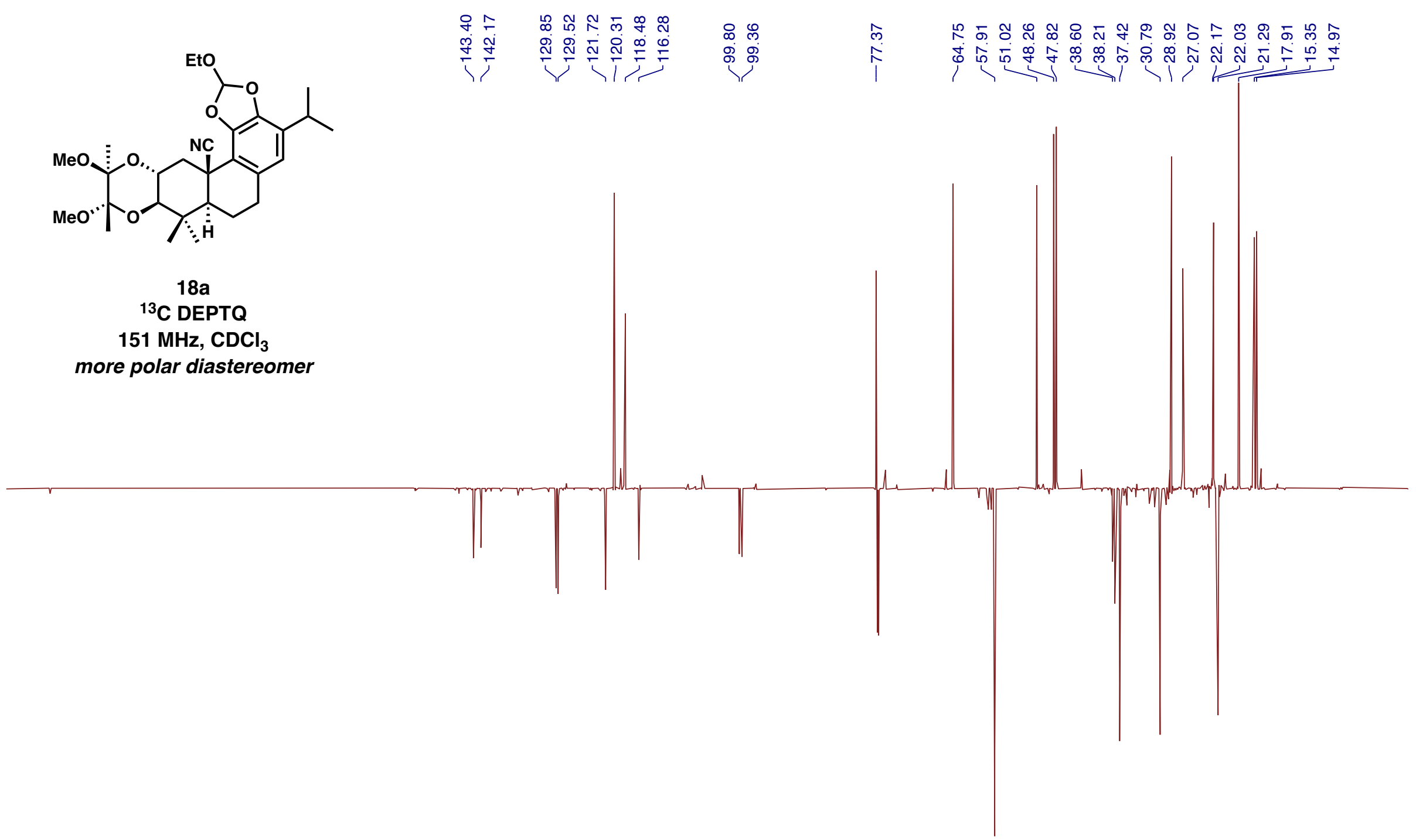

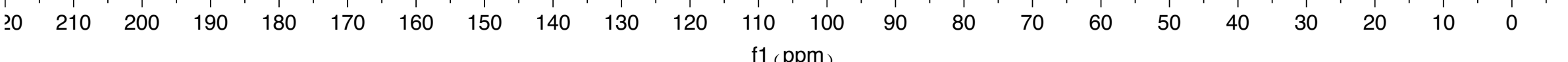


๙ ธ

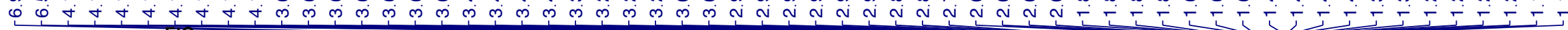

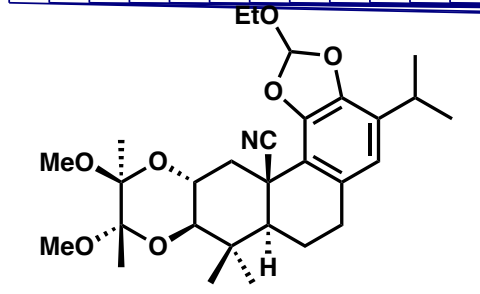

$18 b$

${ }^{1} \mathrm{H}$ NMR

$600 \mathrm{MHz}, \mathrm{CDCl}_{3}$ less polar diastereomer
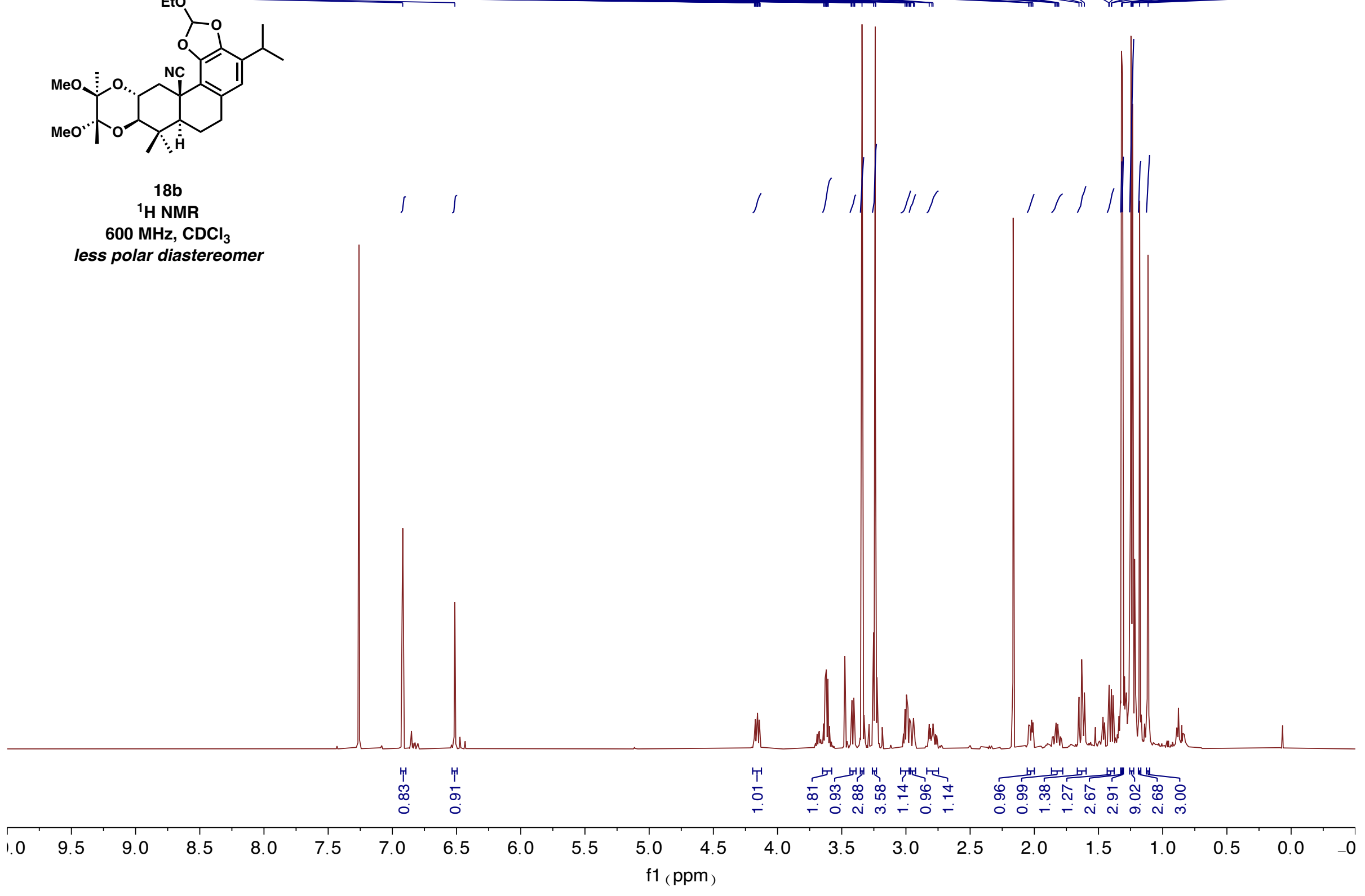

S103 


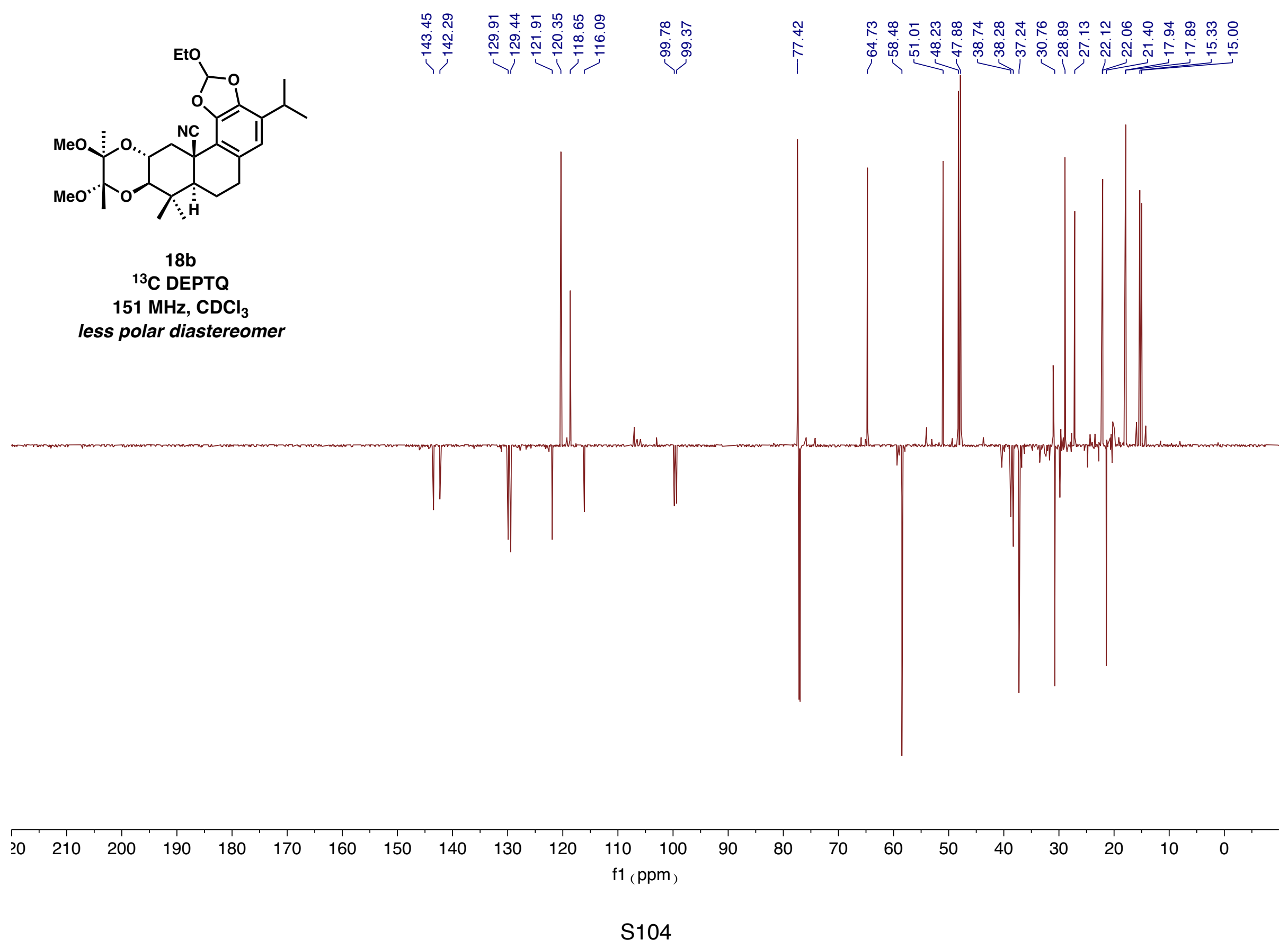




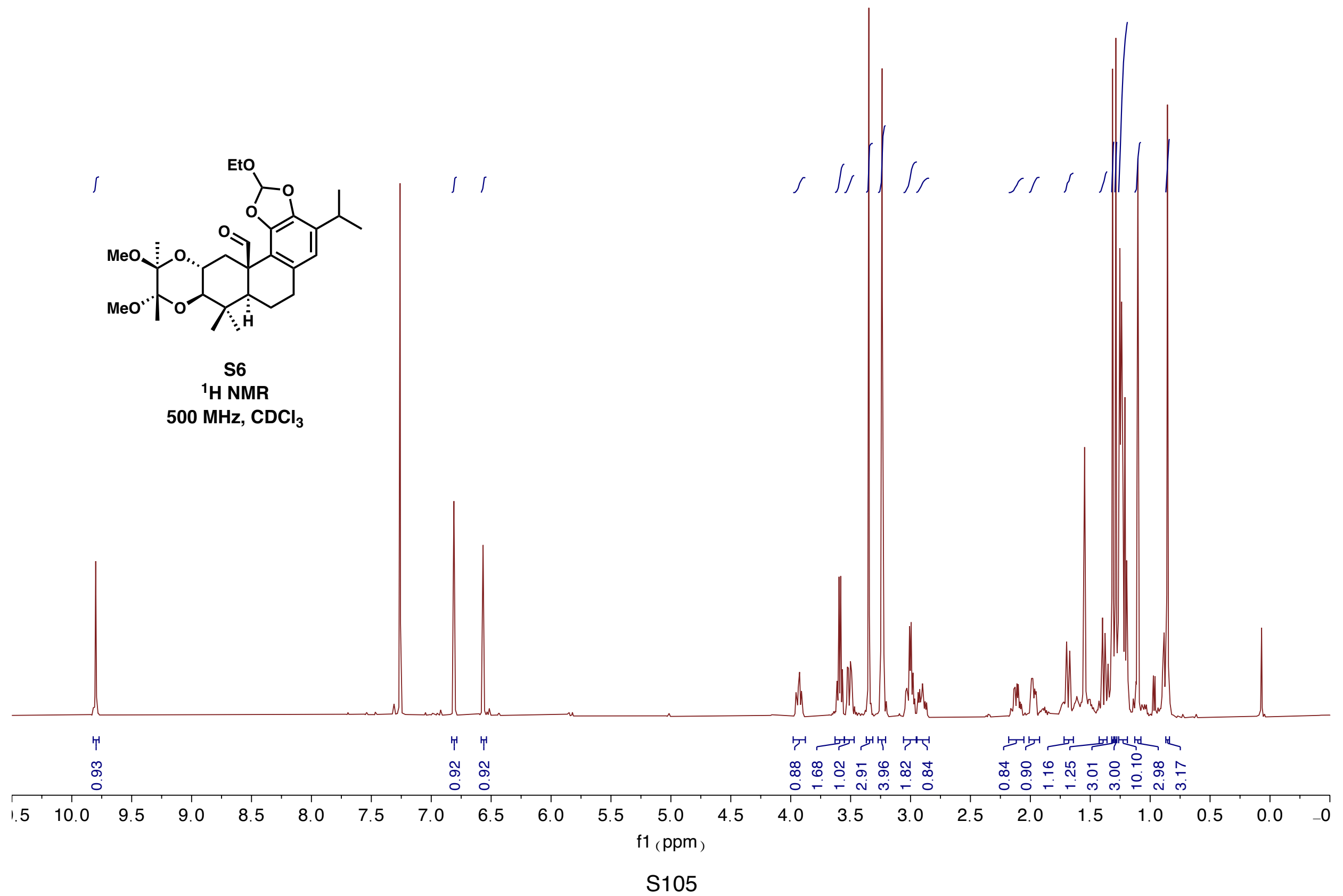




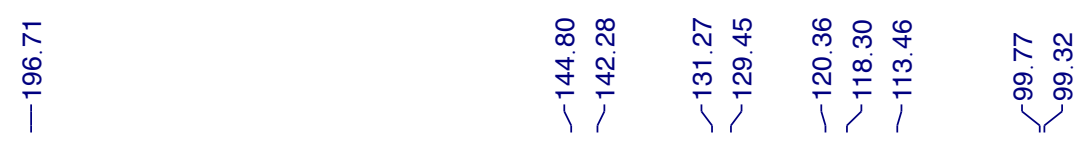

N

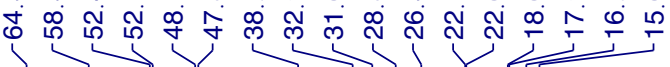

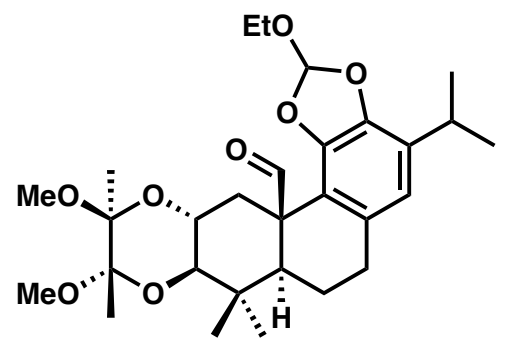

S6

${ }^{13} \mathrm{C}$ NMR

$151 \mathrm{MHz}, \mathrm{CDCl}_{3}$

$\begin{array}{lllllllllllll}20 & 210 & 200 & 190 & 180 & 170 & 160 & 150 & 140 & 130 & 120 & 110 & 100\end{array}$

f1 ( $p p m$ )

S106 
ஹ טं

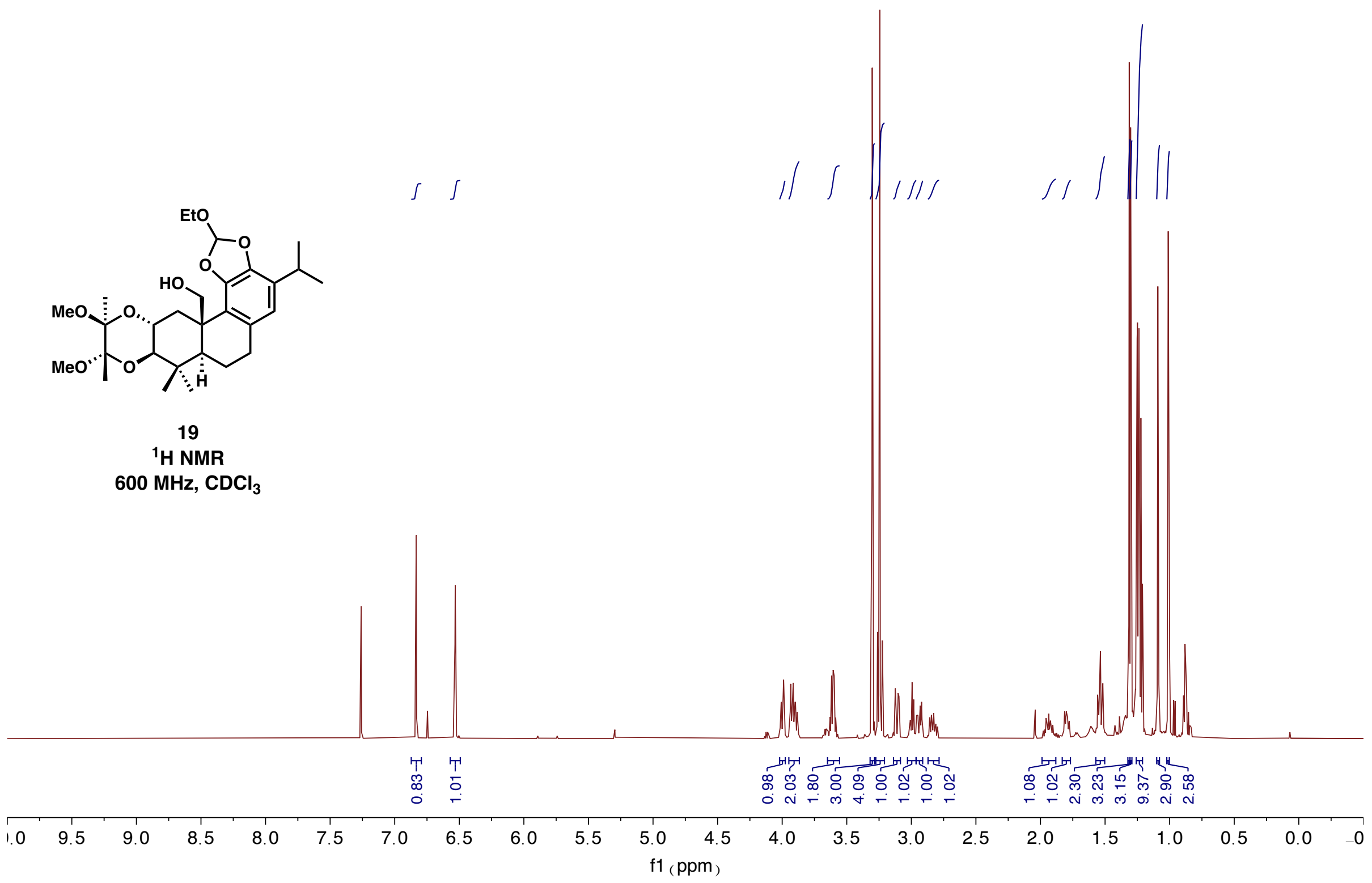




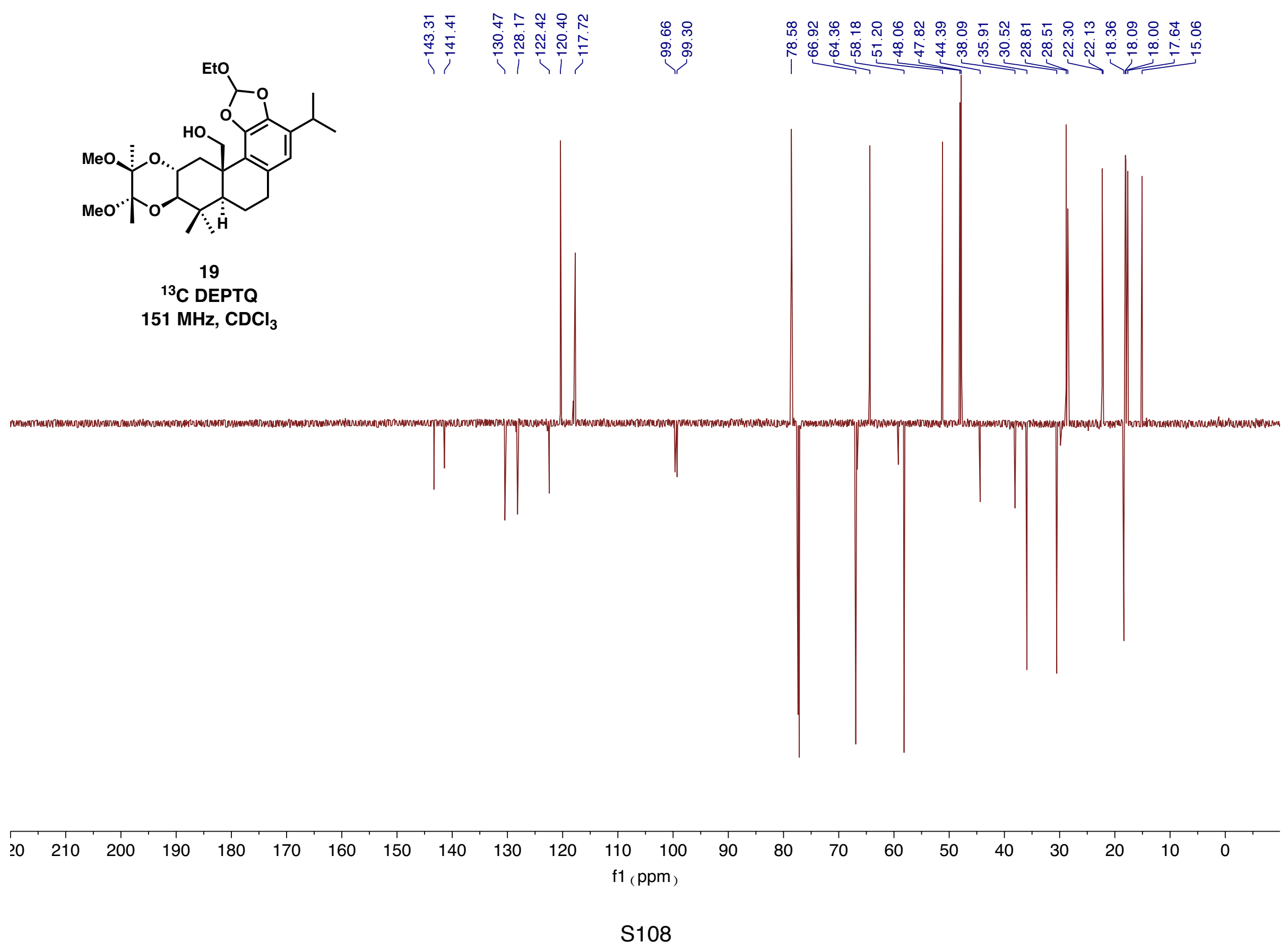




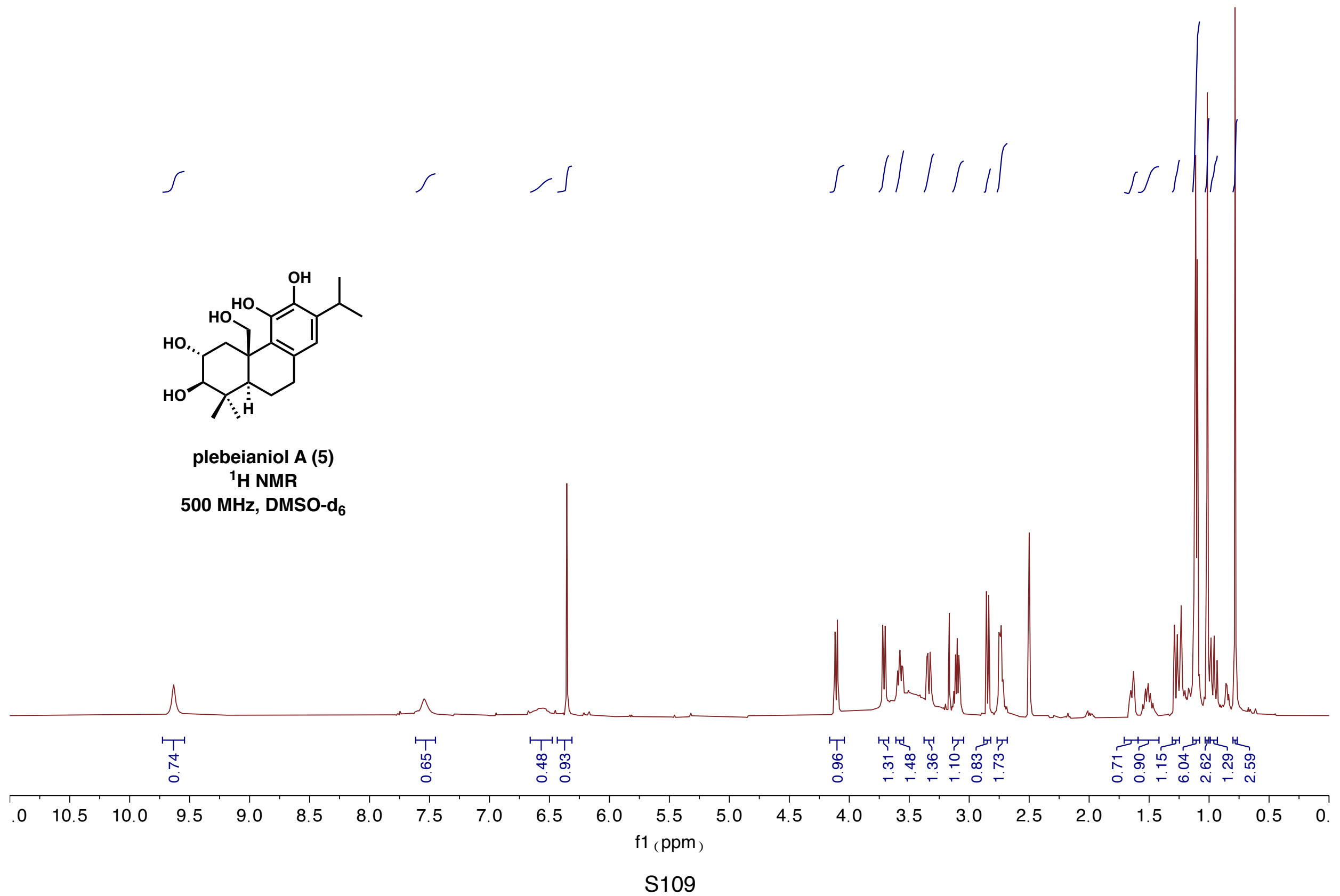




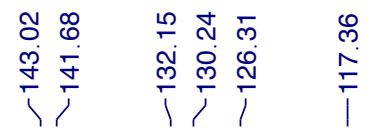<smiles>CC(C)c1cc2c(c(O)c1O)C1(CO)C[C@H](O)[C@@H](O)C(C)(C)[C@H]1CC2</smiles>

plebeianiol A (5)

${ }^{13} \mathrm{C}$ NMR

$151 \mathrm{MHz}, \mathrm{DMSO}^{-\mathrm{d}_{6}}$

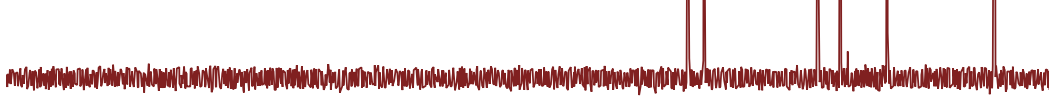

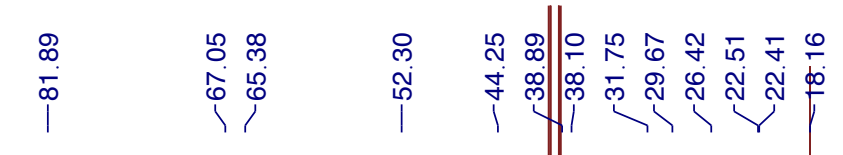

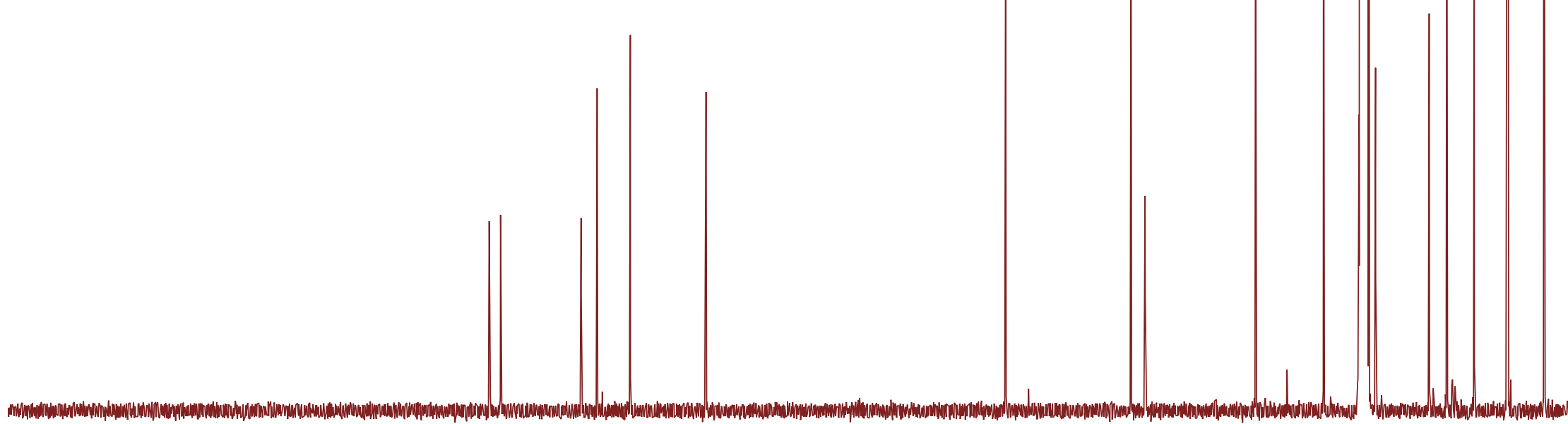

\begin{tabular}{|c|c|c|c|c|c|c|c|c|c|c|c|c|c|c|c|c|c|c|c|}
\hline )0 & 190 & 180 & 170 & 160 & 150 & 140 & 130 & 120 & 110 & $\begin{array}{r}100 \\
\mathrm{f} 1(\mathrm{ppm}\end{array}$ & 90 & 80 & 70 & 60 & 50 & 40 & 30 & 20 & 10 \\
\hline
\end{tabular}




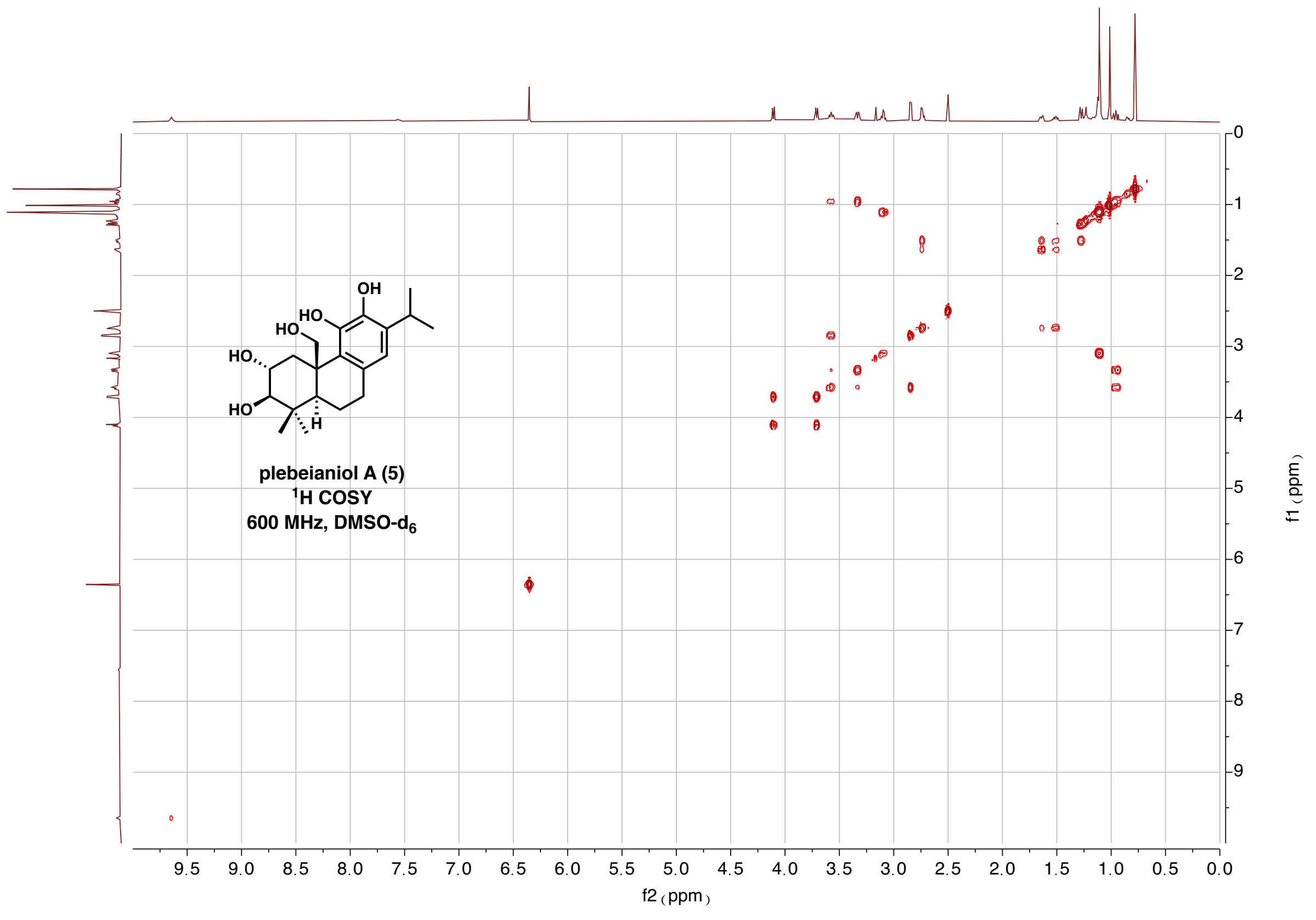

S111 


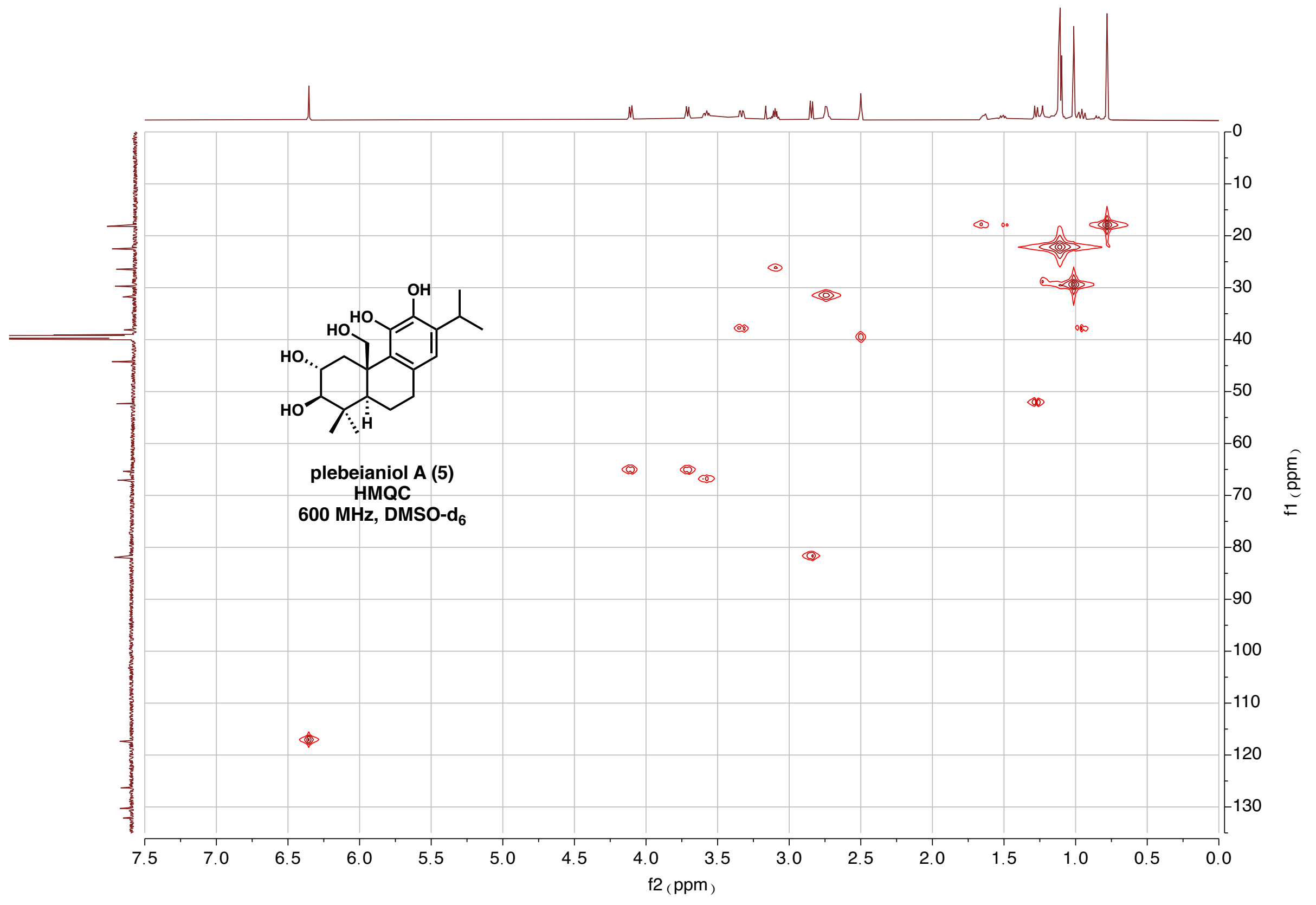

S112 


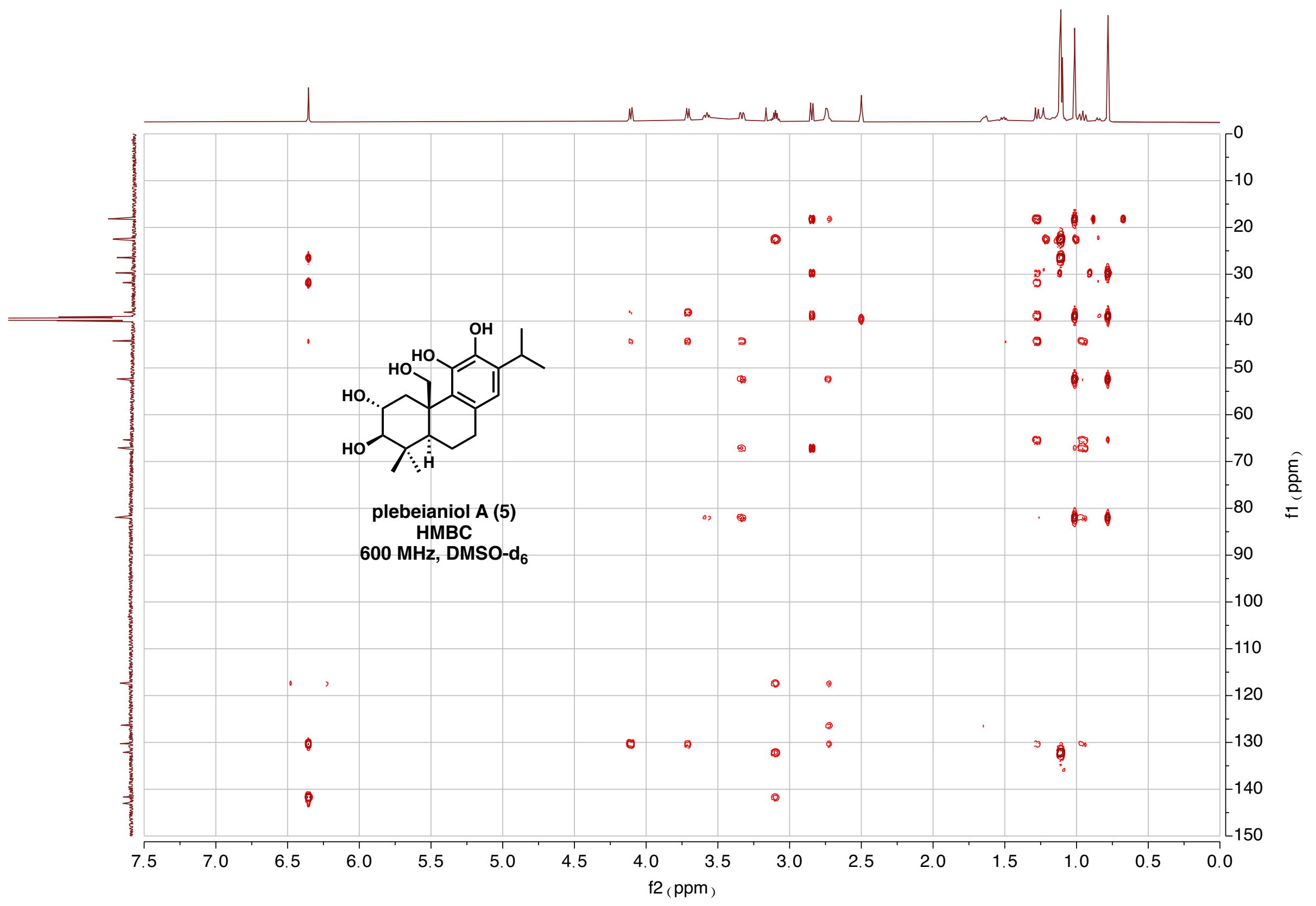

S113 
<smiles>CC(C)c1cc2c(c(O)c1O)C1(C)C[C@H](O)C(O)C(C)(C)[C@@]1(C)C2</smiles>

6: $2 a, 3 \beta, 11,12-t e t r a h y d r o x y-7 \beta$, 20-epoxy-8,11,13-abietatriene ${ }^{1} \mathrm{H}$ NMR

$500 \mathrm{MHz}, \mathrm{DMSO}^{-\mathrm{d}_{6}}$

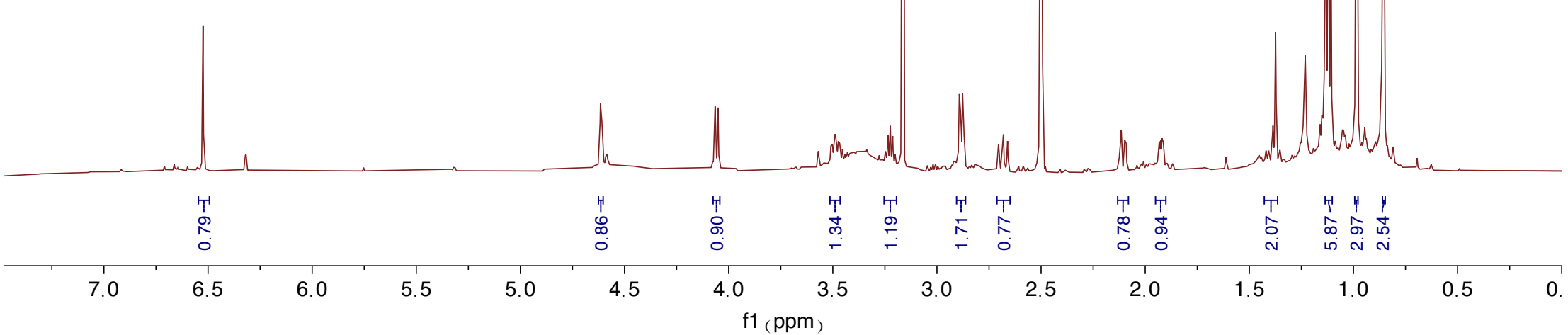




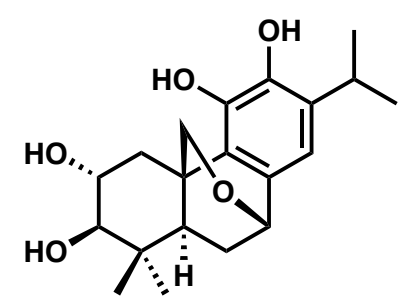

6: $2 a, 3 \beta, 11,12-t e t r a h y d r o x y-7 \beta$, 20-epoxy-8,11,13-abietatriene

${ }^{13} \mathrm{C}$ NMR

$151 \mathrm{MHz}$, DMSO-d $_{6}$

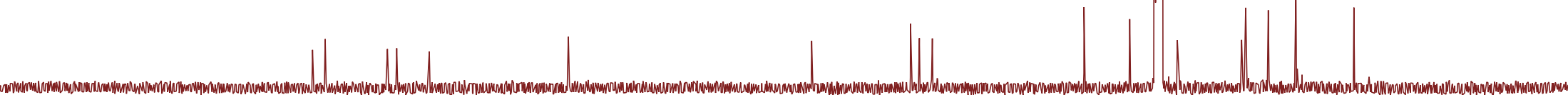

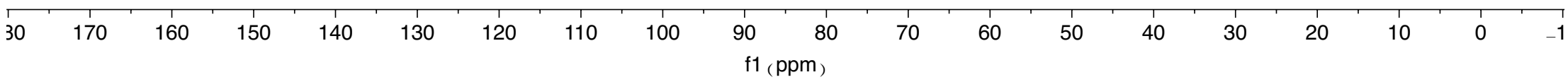




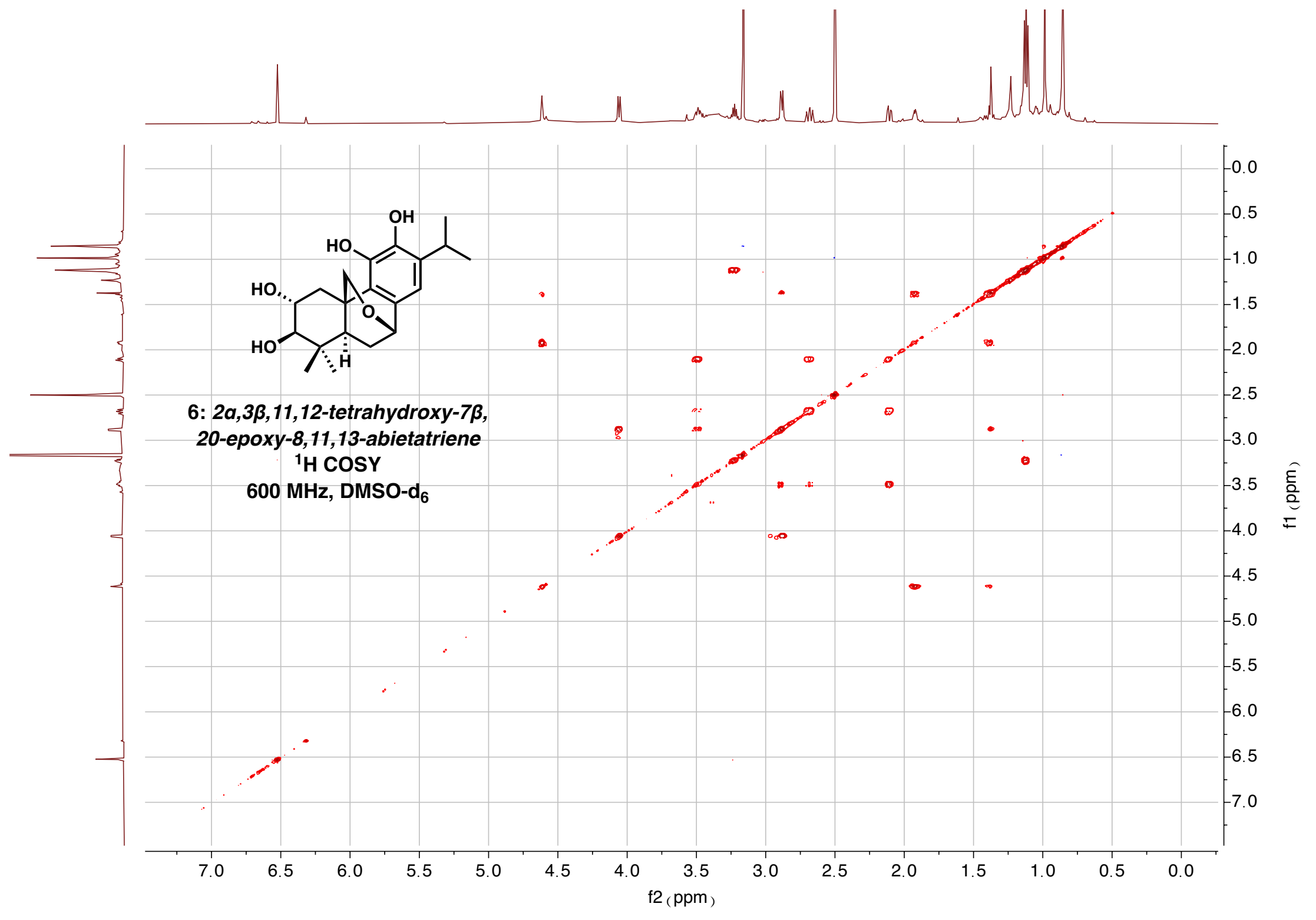

S116 


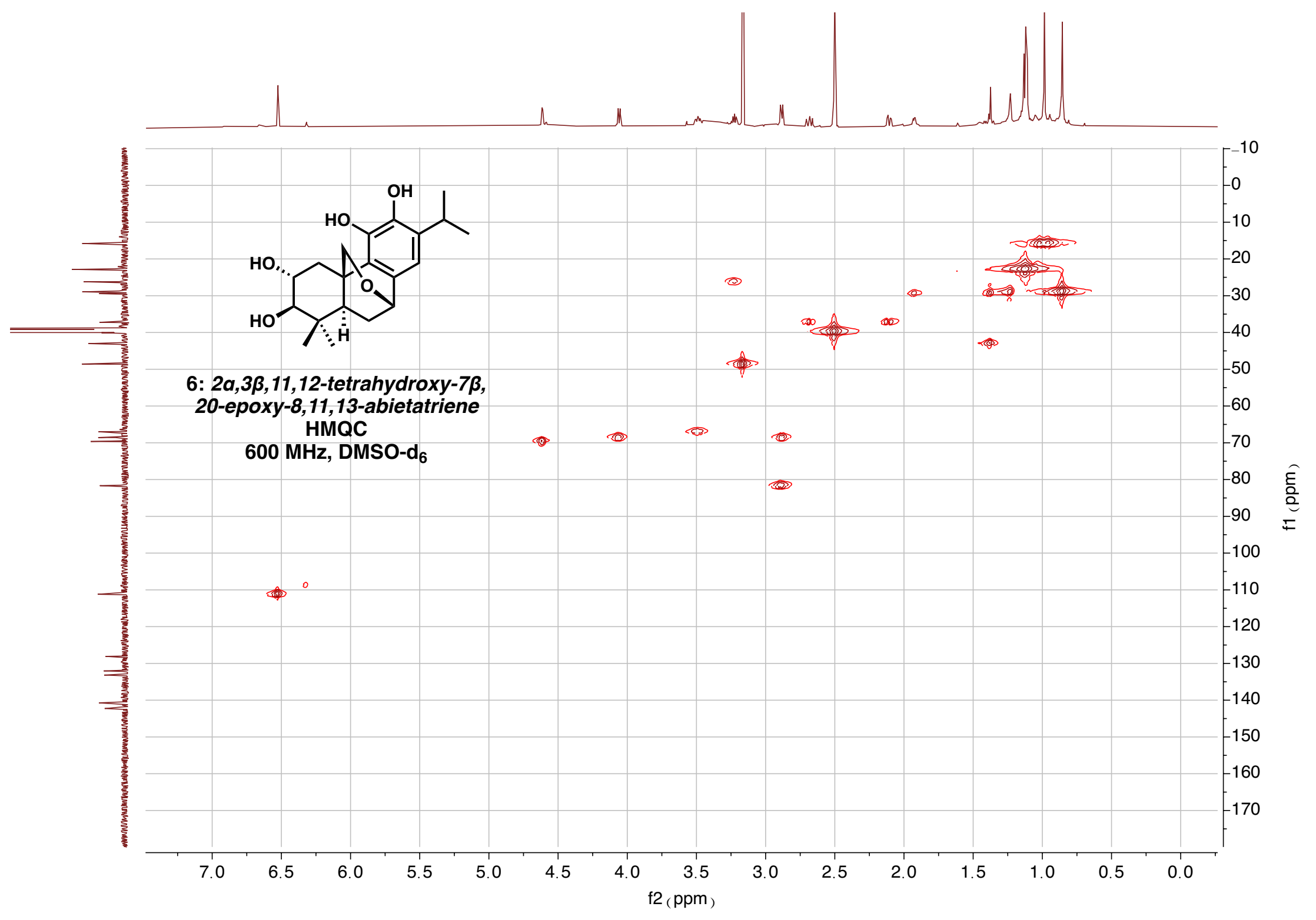




\section{Predicted ${ }^{13} \mathrm{C}$ Shifts and Comparisons of Spectral Data}

Cartesian coordinates for lowest energy conformer of 4 optimized at HF/3-21G

$\begin{array}{llll}\mathrm{C} & -2.278836 & -0.353964 & 0.170491 \\ \mathrm{C} & -0.076068 & 0.530044 & -0.581053 \\ \mathrm{C} & -2.107643 & 0.805708 & -2.026427 \\ \mathrm{C} & -0.730078 & 1.401433 & -1.711113 \\ \mathrm{C} & -2.964381 & 0.596664 & -0.797522 \\ \mathrm{C} & -0.940023 & 0.235104 & 0.688743 \\ \mathrm{C} & -0.182198 & -0.785829 & 1.572755 \\ \mathrm{C} & 1.20174 & -0.979429 & 1.452584 \\ \mathrm{C} & 2.103433 & -0.132166 & 0.542059 \\ \mathrm{C} & 1.326775 & 0.994337 & -0.171507 \\ \mathrm{O} & -4.182752 & 0.019046 & -1.337277 \\ \mathrm{O} & -2.783352 & 1.672695 & -2.950523 \\ \mathrm{C} & 1.846081 & -1.925332 & 2.23755 \\ \mathrm{C} & 1.148729 & -2.699465 & 3.136481 \\ \mathrm{C} & -0.198123 & -2.486106 & 3.306147 \\ \mathrm{C} & -0.850591 & -1.523669 & 2.562911 \\ \mathrm{C} & 2.971641 & -1.021198 & -0.406154 \\ \mathrm{C} & 4.050137 & -0.191289 & -1.134457 \\ \mathrm{C} & 2.178736 & -1.866574 & -1.418404 \\ \mathrm{H} & 0.037987 & -0.445679 & -1.034125 \\ \mathrm{C} & -1.181361 & 1.501882 & 1.536502 \\ \mathrm{O} & -2.224395 & 1.209494 & 2.517369 \\ \mathrm{C} & 0.106141 & 1.255831 & -3.008186 \\ \mathrm{C} & -0.849748 & 2.917574 & -1.439652 \\ \mathrm{O} & -2.17307 & -1.337699 & 2.883537 \\ \mathrm{O} & 1.855555 & -3.630378 & 3.859102 \\ \mathrm{H} & -2.059087 & -1.275329 & -0.357228 \\ \mathrm{H} & -2.937354 & -0.607063 & 0.978607 \\ \mathrm{H} & -1.971156 & -0.175795 & -2.470612 \\ \mathrm{H} & -3.193615 & 1.544115 & -0.328737 \\ \mathrm{H} & 2.816982 & 0.343963 & 1.209774 \\ \mathrm{H} & 1.883738 & 1.315965 & -1.036873 \\ \mathrm{H} & 1.25657 & 1.853422 & 0.485106 \\ \mathrm{H} & -4.822194 & -0.167651 & -0.637923 \\ \mathrm{H} & -3.6915 & 1.349907 & -3.042427 \\ \mathrm{H} & 2.902294 & -2.071488 & 2.173656\end{array}$




$\begin{array}{cccc}\mathrm{H} & -0.773462 & -3.024726 & 4.030137 \\ \mathrm{H} & 3.499529 & -1.713993 & 0.236151 \\ \mathrm{H} & 4.609471 & 0.424008 & -0.436848 \\ \mathrm{H} & 3.616952 & 0.452838 & -1.890116 \\ \mathrm{H} & 4.749395 & -0.855457 & -1.631135 \\ \mathrm{H} & 1.398843 & -2.435051 & -0.927034 \\ \mathrm{H} & 1.73571 & -1.247378 & -2.186751 \\ \mathrm{H} & 2.848588 & -2.566781 & -1.907059 \\ \mathrm{H} & -1.509382 & 2.329665 & 0.938997 \\ \mathrm{H} & -0.272827 & 1.772987 & 2.052944 \\ \mathrm{H} & -2.34677 & 1.93503 & 3.141375 \\ \mathrm{H} & 0.317013 & 0.214311 & -3.221565 \\ \mathrm{H} & -0.468984 & 1.6635 & -3.828842 \\ \mathrm{H} & 1.043621 & 1.794383 & -2.945954 \\ \mathrm{H} & -1.629925 & 3.167771 & -0.737151 \\ \mathrm{H} & -1.102216 & 3.410887 & -2.366593 \\ \mathrm{H} & 0.089532 & 3.318745 & -1.077008 \\ \mathrm{H} & -2.392165 & -0.379096 & 2.868701 \\ \mathrm{H} & 1.29806 & -4.132724 & 4.464575\end{array}$

wB97X-D/6-311+G(2DF,2P) [6-311G*] = -1156.3493933 hartrees

Cartesian coordinates for lowest energy conformer of 5 optimized at HF/3-21G

$\begin{array}{llll}\text { C } & -0.961456 & 2.044844 & 0.086975 \\ \text { C } & 0.307444 & 0.576445 & 1.639934 \\ \text { C } & -0.782555 & 2.667062 & 2.511475 \\ \text { C } & 0.355387 & 1.661668 & 2.764886 \\ \text { C } & -0.817089 & 3.185733 & 1.083373 \\ \text { C } & 0.229236 & 1.058818 & 0.154096 \\ \text { C } & -0.008583 & -0.171469 & -0.753745 \\ \text { C } & 0.246803 & -1.481085 & -0.298914 \\ \text { C } & 0.821193 & -1.773748 & 1.073359 \\ \text { C } & 1.350401 & -0.533494 & 1.781573 \\ \text { C } & -0.032403 & -2.570997 & -1.119804 \\ \text { C } & -0.542353 & -2.44536 & -2.408798 \\ \text { C } & -0.732498 & -1.151921 & -2.88337 \\ \text { C } & -0.449524 & -0.039831 & -2.079238 \\ \text { C } & 1.543178 & 1.736468 & -0.294961 \\ \text { O } & 1.314975 & 2.481142 & -1.498455 \\ \text { H } & -0.667395 & 0.088164 & 1.794542\end{array}$




$\begin{array}{llll}\mathrm{C} & 0.050638 & 0.975915 & 4.113278 \\ \mathrm{C} & 1.702807 & 2.390855 & 2.929598 \\ \mathrm{O} & -0.676267 & 3.738965 & 3.423458 \\ \mathrm{O} & -1.934971 & 4.06783 & 1.040209 \\ \mathrm{O} & -0.654127 & 1.156115 & -2.727829 \\ \mathrm{O} & -1.172234 & -0.939767 & -4.15202 \\ \mathrm{C} & -0.836601 & -3.64945 & -3.28242 \\ \mathrm{C} & 0.449159 & -4.415358 & -3.622221 \\ \mathrm{C} & -1.874703 & -4.574292 & -2.634256 \\ \mathrm{H} & -1.098302 & 2.456074 & -0.912919 \\ \mathrm{H} & -1.879858 & 1.491396 & 0.317676 \\ \mathrm{H} & -1.739864 & 2.136748 & 2.666258 \\ \mathrm{H} & 0.101377 & 3.7573 & 0.881559 \\ \mathrm{H} & 1.609098 & -2.530552 & 0.978693 \\ \mathrm{H} & 0.039695 & -2.224215 & 1.701983 \\ \mathrm{H} & 2.31365 & -0.227804 & 1.355505 \\ \mathrm{H} & 1.540973 & -0.768169 & 2.832303 \\ \mathrm{H} & 0.164022 & -3.56812 & -0.729603 \\ \mathrm{H} & 1.925212 & 2.419234 & 0.464913 \\ \mathrm{H} & 2.301084 & 0.966648 & -0.477092 \\ \mathrm{H} & 2.167067 & 2.686578 & -1.898184 \\ \mathrm{H} & -0.184829 & 1.738809 & 4.860444 \\ \mathrm{H} & -0.807407 & 0.297857 & 4.034481 \\ \mathrm{H} & 0.906046 & 0.402133 & 4.483027 \\ \mathrm{H} & 1.846583 & 3.206062 & 2.216701 \\ \mathrm{H} & 2.540684 & 1.691963 & 2.834057 \\ \mathrm{H} & 1.754223 & 2.845033 & 3.921748 \\ \mathrm{H} & -1.321918 & 4.393047 & 3.121502 \\ \mathrm{H} & -1.939275 & 4.50665 & 0.181672 \\ \mathrm{H} & 0.061456 & 1.766456 & -2.455266 \\ \mathrm{H} & -1.180269 & 0.02428 & -4.26631 \\ \mathrm{H} & -1.259601 & -3.275128 & -4.219507 \\ \mathrm{H} & 0.917387 & -4.821966 & -2.717734 \\ \mathrm{H} & 1.176726 & -3.76095 & -4.112843 \\ \mathrm{H} & 0.233143 & -5.254082 & -4.293826 \\ \mathrm{H} & -1.49742 & -5.002121 & -1.697827 \\ \mathrm{H} & -2.120664 & -5.405415 & -3.30483 \\ \mathrm{H} & -2.797479 & -4.031 & -2.40731\end{array}$

wB97X-D/6-311+G(2DF,2P) $\left[6-311 \mathrm{G}^{*}\right]=-1156.3619383$ hartrees 


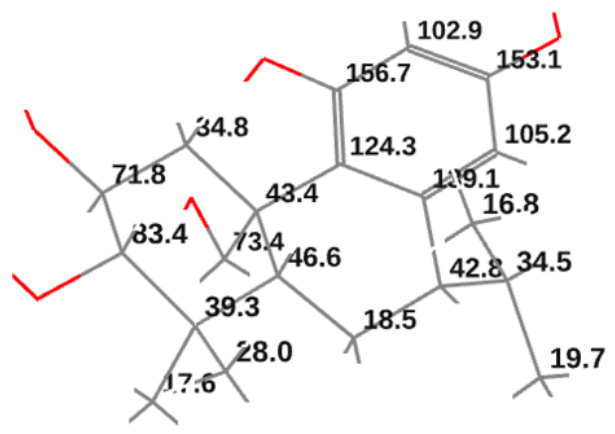

Fig. S2. Plebeianiol A structure 4 optimized at HF/3-21G

Table S1. Isolated experimental and calculated ${ }^{13} \mathrm{C}$ chemical shifts for 4 [these resonances are tabulated in order of decreasing chemical shift, and are the same as those shown in Table 1 of the main paper]

\begin{tabular}{|c|c|c|c|c|}
\hline Carbon \# & Isolated Exp. & $\omega B 97 X-D / 6-31 G *$ & $\Delta$ & $\mathrm{I} \Delta \mathrm{I}$ \\
\hline 1 & 143.0 & 156.7 & -13.7 & 13.7 \\
\hline 2 & 141.6 & 153.1 & -11.5 & 11.5 \\
\hline 3 & 131.9 & 139.1 & -7.2 & 7.2 \\
\hline 4 & 130.2 & 124.3 & 5.9 & 5.9 \\
\hline 5 & 126.3 & 105.2 & 21.1 & 21.1 \\
\hline 6 & 117.5 & 102.9 & 14.6 & 14.6 \\
\hline 7 & 81.8 & 83.4 & -1.6 & 1.6 \\
\hline 8 & 66.8 & 73.4 & -6.6 & 6.6 \\
\hline 9 & 65.3 & 71.8 & -6.5 & 6.5 \\
\hline 10 & 52.1 & 46.6 & 5.5 & 5.5 \\
\hline 11 & 43.8 & 43.4 & 0.4 & 0.4 \\
\hline 12 & 38.6 & 42.8 & -4.2 & 4.2 \\
\hline 13 & 38.0 & 39.3 & -1.3 & 1.3 \\
\hline 14 & 31.7 & 34.8 & -3.1 & 3.1 \\
\hline 15 & 29.6 & 34.5 & -4.9 & 4.9 \\
\hline 16 & 26.4 & 28.0 & -1.6 & 1.6 \\
\hline 17 & 22.4 & 19.7 & 2.7 & 2.7 \\
\hline 18 & 22.3 & 18.5 & 3.8 & 3.8 \\
\hline 19 & 18.1 & 17.6 & 0.5 & 0.5 \\
\hline 20 & 18.1 & 16.8 & 1.3 & 1.3 \\
\hline
\end{tabular}

Average I $\Delta \mathrm{I}: 5.9$ $\operatorname{Max}$ I $\Delta \mathrm{I}: 21.1$ 


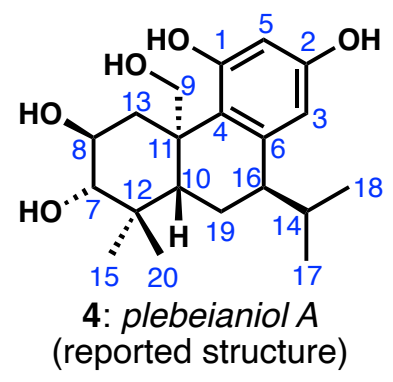

Table S2. Isolated experimental and calculated ${ }^{13} \mathrm{C}$ chemical shifts for 4 [these resonances are tabulated according to the assignments made by Liang, $\mathrm{Wu}$, and co-workers, in order of decreasing chemical shift for the reported resonances]

\begin{tabular}{r|r|r|r|r} 
Carbon \# & Isolated Exp. & $\omega B 97 X-D / 6-31 G *$ & $\Delta$ & $I \Delta$ I \\
\hline $\mathbf{1}$ & 143.0 & 156.7 & -13.7 & 13.7 \\
2 & 141.6 & 153.1 & -11.5 & 11.5 \\
3 & 131.9 & 105.2 & 26.7 & 26.7 \\
4 & 130.2 & 124.3 & 5.9 & 5.9 \\
5 & 126.3 & 102.9 & 23.4 & 23.4 \\
6 & 117.5 & 139.1 & -21.6 & 21.6 \\
7 & 81.8 & 83.4 & -1.6 & 1.6 \\
8 & 66.8 & 71.8 & -5 & 5 \\
9 & 65.3 & 73.4 & -8.1 & 8.1 \\
10 & 52.1 & 46.6 & 5.5 & 5.5 \\
11 & 43.8 & 43.4 & 0.4 & 0.4 \\
12 & 38.6 & 39.3 & -0.7 & 0.7 \\
13 & 38.0 & 34.8 & 3.2 & 3.2 \\
14 & 31.7 & 34.5 & -2.8 & 2.8 \\
15 & 29.6 & 28.0 & 1.6 & 1.6 \\
16 & 26.4 & 42.8 & -16.4 & 16.4 \\
17 & 22.4 & 19.7 & 2.7 & 2.7 \\
18 & 22.3 & 16.8 & 5.5 & 5.5 \\
19 & 18.1 & 18.5 & -0.4 & 0.4 \\
20 & 18.1 & 16.8 & 1.3 & 1.3 \\
& & & &
\end{tabular}

Average I $\Delta \mathrm{I}: 7.9$ $\operatorname{Max}$ I $\Delta \mathrm{I}: 26.7$ 


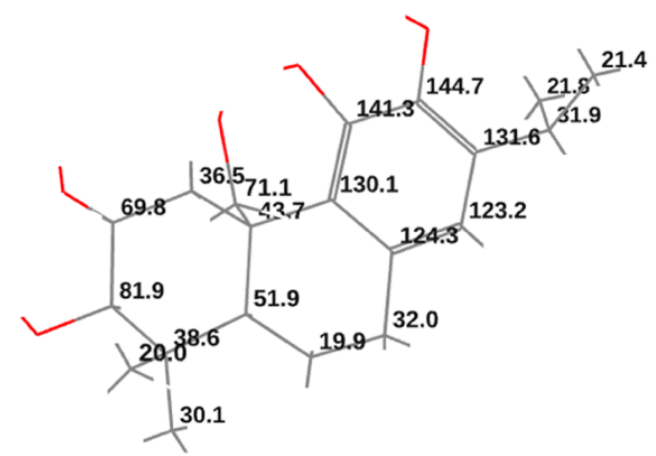

Fig. S3. Plebeianiol A structure 5 optimized at $\mathrm{HF} / 3-21$

Table S3. Synthetic experimental and calculated ${ }^{13} \mathrm{C}$ chemical shifts for 5 [these resonances are tabulated in order of decreasing chemical shift, and are the same as those shown in Table 1 of the main paper]

\begin{tabular}{r|r|r|r|r} 
Carbon \# & Synthetic Exp. & $\boldsymbol{\omega}$ B97X-D/6-31G* & $\boldsymbol{\Delta}$ & $\mathbf{I} \boldsymbol{\Delta}$ I \\
\hline 1 & 143.0 & 144.7 & -1.7 & 1.7 \\
2 & 141.6 & 141.3 & 0.3 & 0.3 \\
3 & 132.2 & 131.6 & 0.6 & 0.6 \\
4 & 130.2 & 130.1 & 0.1 & 0.1 \\
5 & 126.3 & 124.3 & 2.0 & 2.0 \\
6 & 117.4 & 123.2 & -5.8 & 5.8 \\
7 & 81.9 & 81.9 & 0.0 & 0.0 \\
8 & 67.1 & 71.1 & -4.0 & 4.0 \\
9 & 65.4 & 69.8 & -4.4 & 4.4 \\
10 & 52.3 & 51.9 & 0.4 & 0.4 \\
11 & 44.3 & 43.7 & 0.6 & 0.6 \\
12 & 38.9 & 38.6 & 0.3 & 0.3 \\
13 & 38.1 & 36.5 & 1.6 & 1.6 \\
14 & 31.8 & 32.0 & -0.2 & 0.2 \\
15 & 29.7 & 31.9 & -2.2 & 2.2 \\
16 & 26.4 & 30.1 & -3.7 & 3.7 \\
17 & 22.5 & 21.8 & 0.7 & 0.7 \\
18 & 22.4 & 21.4 & 1.0 & 1.0 \\
19 & 18.2 & 20.0 & -1.8 & 1.8 \\
20 & 18.2 & 19.9 & -1.7 & 1.7
\end{tabular}

Average I $\Delta \mathrm{I}: 1.7$ $\operatorname{Max} \mid \Delta \mathrm{I}: 5.8$ 


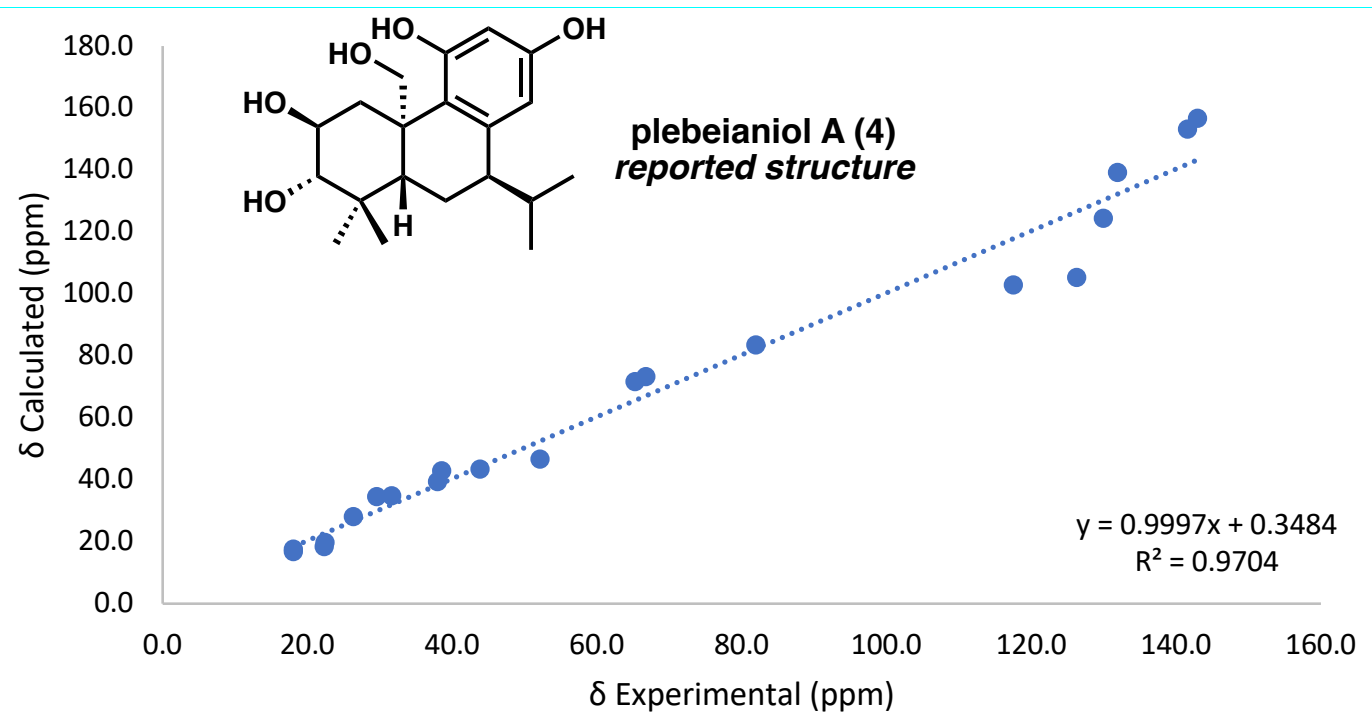

Fig. S4. Linear correlation plot for 4 [based on data from Table S1]

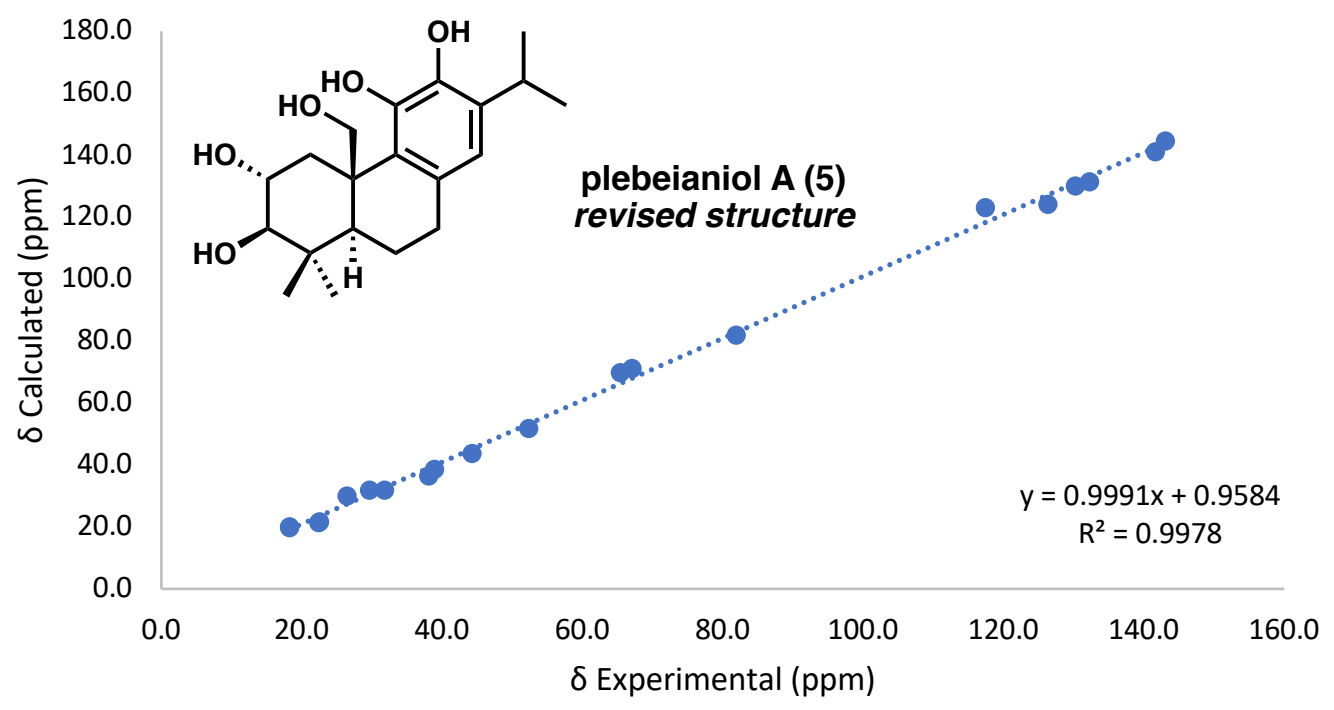

Fig. S5. Linear correlation plot for 5 
Table S4. Comparison of isolated experimental and synthetic experimental ${ }^{13} \mathrm{C}$ chemical shifts for plebeianiol A in DMSO-d6

\begin{tabular}{r|r|r|r|r} 
Carbon \# & Isolated & Synthetic & $\boldsymbol{\Delta}$ & $\mathbf{I} \boldsymbol{\Delta}$ I \\
\hline 1 & 143.0 & 143.0 & 0.0 & 0.0 \\
2 & 141.6 & 141.6 & 0.0 & 0.0 \\
3 & 131.9 & 132.2 & -0.3 & 0.3 \\
4 & 130.2 & 130.2 & 0.0 & 0.0 \\
5 & 126.3 & 126.3 & 0.0 & 0.0 \\
6 & 117.5 & 117.4 & 0.1 & 0.1 \\
7 & 81.8 & 81.9 & -0.1 & 0.1 \\
8 & 66.8 & 67.1 & -0.3 & 0.3 \\
9 & 65.3 & 65.4 & -0.1 & 0.1 \\
10 & 52.1 & 52.3 & -0.2 & 0.2 \\
11 & 43.8 & 44.3 & -0.5 & 0.5 \\
12 & 38.6 & 38.9 & -0.3 & 0.3 \\
13 & 38.0 & 38.1 & -0.1 & 0.1 \\
14 & 31.7 & 31.8 & -0.1 & 0.1 \\
15 & 29.6 & 29.7 & -0.1 & 0.1 \\
16 & 26.4 & 26.4 & 0.0 & 0.0 \\
17 & 22.4 & 22.5 & -0.1 & 0.1 \\
18 & 22.3 & 22.4 & -0.1 & 0.1 \\
19 & 18.1 & 18.2 & -0.1 & 0.1 \\
20 & 18.1 & 18.2 & -0.1 & 0.1 \\
& & & &
\end{tabular}

Average I $\Delta \mathrm{I}: 0.1$

Max I $\Delta$ I: 0.5 


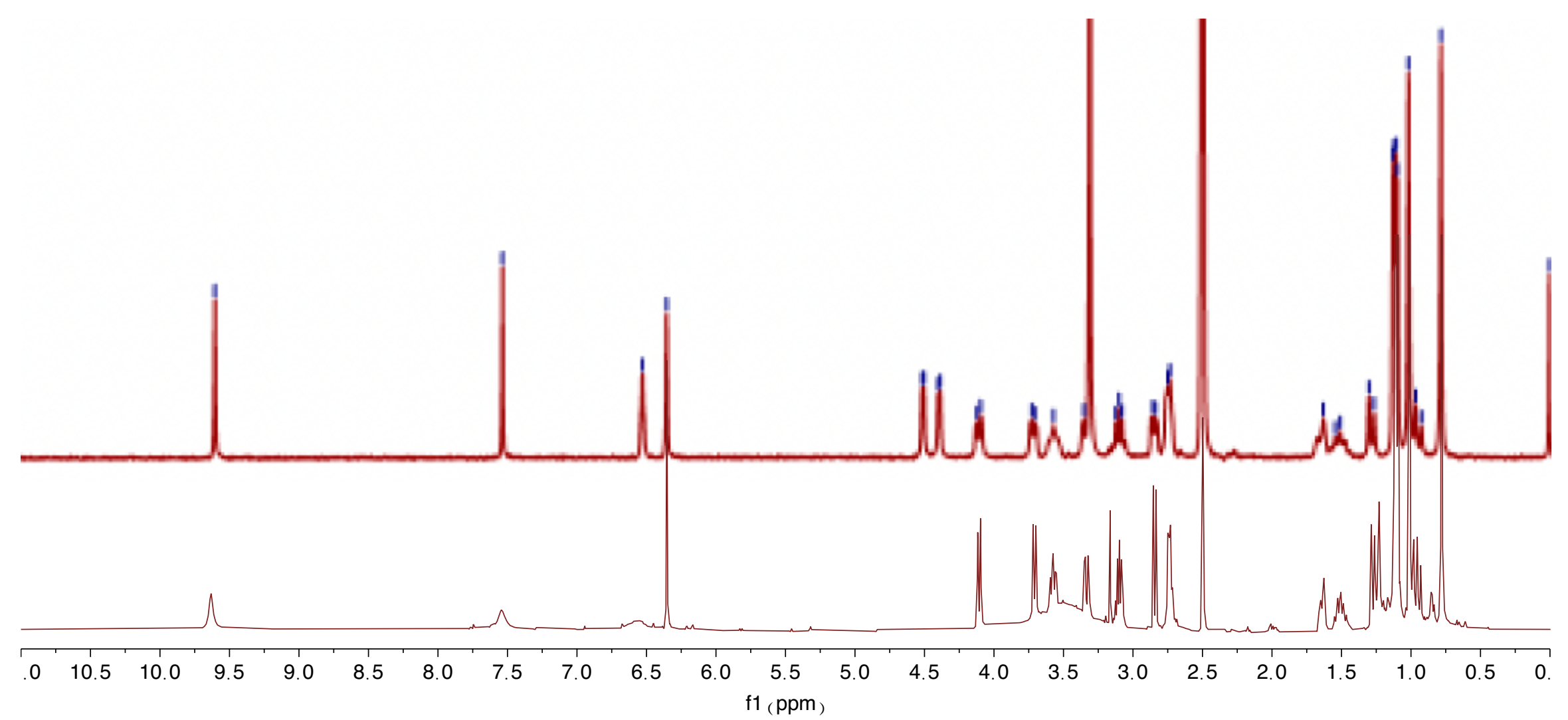

Fig. S6. Comparison of ${ }^{1} \mathrm{H}$ NMR spectrum of isolated (top) plebeianiol A versus synthetic (bottom) plebeianiol A. Please note that the ${ }^{1} \mathrm{H}$ NMR spectrum for synthetic plebeianiol A displays broadening of the catechol O-H's and primary alcohol $\mathrm{O}-\mathrm{H}$ at $9.63 \mathrm{ppm}, 7.54 \mathrm{ppm}$, and $6.36 \mathrm{ppm}$. Also note that the exchangeable $\mathrm{O}-\mathrm{H}$ protons of the secondary alcohols reported at $4.51 \mathrm{ppm}$ and $4.39 \mathrm{ppm}$ are absent. 


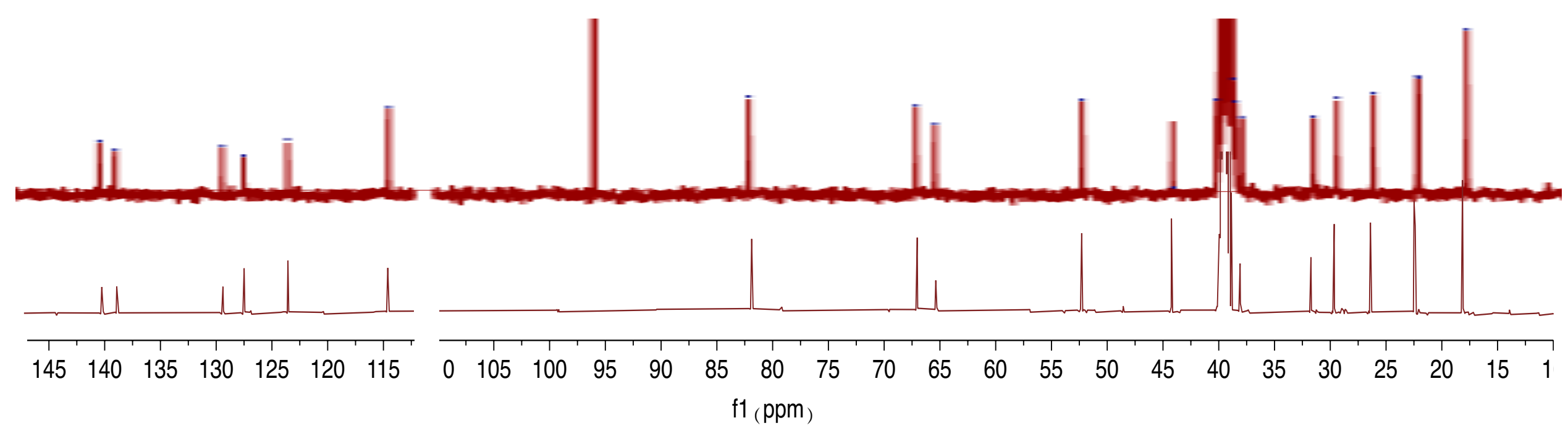

Fig. S7. Comparison of ${ }^{13} \mathrm{C}$ NMR spectrum of isolated (top) plebeianiol A versus synthetic (bottom) plebeianiol A. Please note the presence of an unreported solvent/impurity observed at approximately $95 \mathrm{ppm}$ in the ${ }^{13} \mathrm{C}$ NMR spectrum for isolated plebeianiol A. 